\title{
Datenreport \\ Erziehungswissenschaft 2020
}

Hermann Josef Abs, Harm Kuper,

Renate Martini (Hrsg.)

Schriften der

Deutschen Gesellschaft für Erziehungswissenschaft

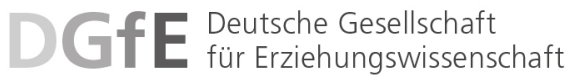

Verlag Barbara Budrich 
Datenreport Erziehungswissenschaft 2020 
Schriften der Deutschen Gesellschaft für Erziehungswissenschaft (DGfE) 


\section{Hermann Josef Abs \\ Harm Kuper \\ Renate Martini (Hrsg.)}

\section{Datenreport \\ Erziehungswissenschaft 2020}

Erstellt im Auftrag

der Deutschen Gesellschaft für Erziehungswissenschaft (DGfE)

Verlag Barbara Budrich

Opladen • Berlin • Toronto 2020 


\section{Dieser Datenreport wurde aus Mitteln der Max-Traeger-Stiftung gefördert.}

Bibliografische Information der Deutschen Nationalbibliothek

Die Deutsche Nationalbibliothek verzeichnet diese Publikation in der Deutschen Nationalbibliografie; detaillierte bibliografische Daten sind im Internet über http://dnb.d-nb.de abrufbar.

(C) 2020 Dieses Werk ist bei der Verlag Barbara Budrich GmbH erschienen und steht unter der Creative Commons Lizenz Attribution-ShareAlike 4.0 International (CC BY-SA 4.0): https://creativecommons.org/licenses/by-sa/4.0/.

Diese Lizenz erlaubt die Verbreitung, Speicherung, Vervielfältigung und Bearbeitung bei Verwendung der gleichen CC-BY-SA 4.0-Lizenz und unter Angabe der UrheberInnen, Rechte, Änderungen und verwendeten Lizenz. www.budrich.de

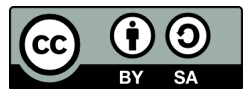

Dieses Buch steht im Open-Access-Bereich der Verlagsseite zum kostenlosen Download bereit (https://doi.org/10.3224/84742419).

Eine kostenpflichtige Druckversion (Print on Demand) kann über den Verlag bezogen werden. Die Seitenzahlen in der Druck- und Onlineversion sind identisch.

$$
\begin{array}{ll}
\text { ISBN } & 978-3-8474-2419-2 \text { (Paperback) } \\
\text { eISBN } & 978-3-8474-1551-0 \text { (PDF) } \\
\text { DOI } & 10.3224 / 84742419
\end{array}
$$

Das Werk einschließlich aller seiner Teile ist urheberrechtlich geschützt. Jede Verwertung außerhalb der engen Grenzen des Urheberrechtsgesetzes ist ohne Zustimmung des Verlages unzulässig und strafbar. Das gilt insbesondere für Vervielfältigungen, Übersetzungen, Mikroverfilmungen und die Einspeicherung und Verarbeitung in elektronischen Systemen.

Umschlaggestaltung: Bettina Lehfeldt, Kleinmachnow - www.lehfeldtgraphic.de Satz: Judith Zimmer, Hamburg - www.lektorenzimmer.de Druck: paper \& tinta, Warschau Printed in Europe 


\section{Inhalt}

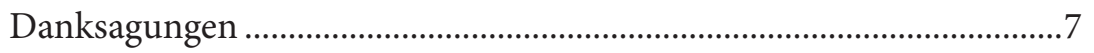

Datenreport Erziehungswissenschaft -

zwanzig Jahre indikatorenbasiertes Monitoring der Disziplin...............9

Hermann Josef Abs \& Harm Kuper

1 Studiengänge und Standorte im Hauptfach..................................... 21

Cathleen Grunert, Katja Ludwig \& Kilian Hüfner

1.1 Studiengänge und Standorte im Überblick

1.2 Studienfachbezeichnungen

1.3 Studieninhalte

1.4 Studiengangsmuster

1.5 Fazit

2 Studiengänge und Standorte der

Lehrerinnen- und Lehrerbildung .................................................... 51

Melanie Radhoff \& Christiane Ruberg

2.1 Strukturvarianten in der Lehrerinnen- und Lehrerbildung

2.2 Standorte Lehramtsstudiengänge

2.3 Bedarfsprognosen

2.4 Ausblick: Kontinuitäten und Diskontinuitäten

3 Studienabschlüsse, Übergänge und beruflicher Verbleib der Absolventinnen und Absolventen .............................................79

Christian Kerst \& Andrä Wolter

3.1 Abschlussprüfungen im Spiegel der amtlichen Statistik

3.2 Übergänge und Verbleib der Absolventinnen und Absolventen

3.3 Merkmale der Erwerbstätigkeit in mittelfristiger Perspektive

3.4 Zusammenfassung der Ergebnisse im Kontext der bisherigen Datenreporte

4 Personal .115

Marius Gerecht, Heinz-Hermann Krüger, Markus Sauerwein é Johanna Schultheiß

4.1 Erziehungswissenschaftliches Personal im Innen- und Außenvergleich

4.2 Personalentwicklung im Fach Erziehungswissenschaft aus der Perspektive von Stellenausschreibungen

4.3 Geschlechterverhältnisse in den Erziehungswissenschaften

4.4 Fazit 
$5 \quad$ Forschung und Publikationskulturen ............................................... 147 Bernhard Schmidt-Hertha \& Margaretha Müller

5.1 Forschungsförderung und Drittmittel

5.2 Publikationskulturen in der Erziehungswissenschaft

5.3 Fazit

6 Habilitationen und Promotionen in der Erziehungswissenschaft.....

Renate Martini

6.1 Habilitationen und Promotionen in der Zeitschrift für Pädagogik

6.2 Promotionen und Habilitationen im Fächervergleich

6.3 Fazit

7 Der Stellenmarkt von wissenschaftlichem Nachwuchs in Erziehungswissenschaft/Bildungsforschung

Annette Stelter

7.1 Methoden

7.2 Umfang und Inhalt der Stellenausschreibungen

7.3 Weiterqualifikationsmöglichkeiten für wissenschaftlichen Nachwuchs

7.4 Zusammenfassung und Fazit der Analyse der Stellenausschreibungen

Tabellenanhang. 197

Die Autorinnen und Autoren 


\section{Danksagungen}

Zum Gelingen dieses Datenreports haben neben den Autorinnen und Autoren all diejenigen beigetragen, die einschlägige Daten erhoben, archiviert, ausgewertet und zur Verfügung gestellt haben. In jeder Hinsicht als verlässlich hat sich auch in diesem Datenreport wieder die Arbeit des Statistischen Bundesamts erwiesen. Frau Brigitte Damm sowie Herrn Thomas Weise gilt daher ein besonderer Dank. Wie so oft in der Bildungsberichterstattung blieb zwischen der Veröffentlichung der aktuellsten Daten und dem Abgabetermin für das Manuskript nur ein kleines Zeitfenster. Es wurde von allen Beteiligten ohne Scheu vor Terminstress genutzt.

Für ein professionelles Lektorat und ein sorgfältiges Layout danken wir Judith Zimmer, deren Einsatz mit dem Schwinden besagten Zeitfensters immer größer wurde und die letzte Hand an das druckfertige Manuskript angelegt hat, bevor es in die bewährten Hände des Verlags Barbara Budrich gegeben werden konnte.

Die Max-Traeger-Stiftung hat die Publikation des Datenreports finanziell großzügig gefördert.

Hermann Josef Abs

Harm Kuper

Renate Martini 



\title{
Datenreport Erziehungswissenschaft - zwanzig Jahre indikatorenbasiertes Monitoring der Disziplin
}

\author{
Hermann Josef Abs \& Harm Kuper
}

Der vorliegende Datenreport Erziehungswissenschaft 2020 ist der bislang sechste. Er setzt eine bewährte Reihe fort und markiert ein Jubiläum - in ihrem vierjährigen Berichtsrhythmus umfassen die Datenreporte nunmehr einen Zeitraum von zwanzig Jahren. Sie dokumentieren die Entwicklung der Erziehungswissenschaft anhand quantitativer sowie qualitativer Indikatoren und begleiten deren strukturelle Veränderungen seit dem Jahr 2000. Die Erziehungswissenschaft verdankt die außerordentlich differenzierte Informiertheit über Bestand und Veränderungen ihrer Leistungen in Forschung und Lehre sowie über ihre Ausstattung an den Universitäten dem Datenreport. Konnten die Initiatoren des Projekts noch feststellen, der $»$ Datenreport liefert [...] neue, bisweilen so nicht bekannte Einsichten und zwingt letztlich auch zur Revision mancher Vorurteile« (Krüger et al. 2000: 11), so kann sich heute niemand mehr bei Betrachtungen zur Erziehungswissenschaft überrascht zeigen oder subjektive Auffassungen über die Struktur des Faches unbegründet lassen. Der Datenreport klärt auf, bietet ein solides empirisches Fundament zur Diskussion von Bewertungen und Handlungserfordernissen. Dabei bietet der Datenreport nicht in jeder seiner Folgen im engeren Sinn »neue « Informationen - er zeigt die Trends auf, in denen sich Studierende, Studiengänge, Personal, Nachwuchs und Leistungen entwickeln. Wandel wird dabei ebenso deutlich wie Kontinuität. Anstelle von Neuigkeit ist Aktualität der Information also das Kriterium, an dem sich der Datenreport messen lassen muss.

Der Zeitpunkt zum Beginn eines regelmäßigen Datenreports hätte kaum günstiger gewählt werden können. Das Jahr 2000 steht am Anfang maßgeblicher struktureller Veränderungen des Hochschulsystems, die auf alle Fächer und somit auch auf die Erziehungswissenschaft einen erheblichen Einfluss hatten. Im Jahr 1999 unterzeichneten Bildungsminister europäischer Länder die BolognaVereinbarung, mit der ein bis zum Jahre 2010 europaweit umzusetzender Umbau der Studienstrukturen nach einem gestuften Modell intendiert war. Für die Erziehungswissenschaft war damit nicht nur der Abschied von Diplom- und Magisterstudiengängen verbunden, sie spielte auch eine zentrale Rolle bei der Umstellung von Lehramtsstudiengängen auf die konsekutive Struktur. Das dem ersten Datenreport noch zugrunde liegende »Qualifikationsdreieck >Diplom-MagisterLehramt « (Otto/Zedler 2000: 16) wurde bald mit erheblichen Folgen für die Studienstruktur in die Bachelor/Master-Struktur überführt. Aber auch quantitativ 
verändert sich das Hochschulsystem in diesen Jahren in einer Weise, die qualitative Veränderungen zur Folge hat: Gegenüber der Gesamtzahl der Studierenden an deutschen Hochschulen im Jahr 2000 nimmt die Zahl bis zum Jahr 2018 um über die Hälfte zu. Die im Datenreport Erziehungswissenschaft dokumentierten Jahre sind somit für die Hochschulen insgesamt eine Zeit beispielloser Bildungsexpansion. Das macht sich auch beim Personal bemerkbar. Über alle Fächer und Stellenkategorien hinweg erfolgt im genannten Zeitraum fast eine Verdopplung des Personals. Allerdings erfolgt diese Veränderung nicht durchgängig im Sinne eines linearen Aufbaus aller Beschäftigtengruppen. Die Zahl ${ }^{1}$ der an den Hochschulen tätigen Professorinnen und Professoren aller Fächer wächst zwar von fast $38.000 \mathrm{im}$ Jahr 2000 auf fast $48.000 \mathrm{im}$ Jahr 2018. Allerdings werden ab dem Jahre 2003 - in dem die Umsetzung einer Besoldungsreform und die Einführung der Juniorprofessuren einsetzte - ehemals in den Personalstatistiken gesondert ausgewiesene Assistentenstellen in die Personalkategorie »Professur « verlagert. Die Summe der Stellen beider Kategorien liegt in den beiden Jahren bei knapp 52.000. Massive Zuwächse erfolgen jedoch in den Personalkategorien des sogenannten wissenschaftlichen Mittelbaus. Die Zahl der wissenschaftlichen Mitarbeitenden hat sich seit 2000 von knapp 98.000 auf 193.000 nahezu verdoppelt; die Zahl der Lehrkräfte für besondere Aufgaben steigt von ca. 6.500 auf 10.300 und die der Lehrbeauftragten gar von knapp 45.000 auf beinahe 99.000. Die Expansion des Studienbetriebs an den Hochschulen erfolgt somit mehrheitlich mit Stellen, deren Inhaberinnen und Inhaber entweder selber noch in der wissenschaftlichen Qualifikation befindlich sind, aufgrund der Fokussierung auf Lehre selbst kaum Forschung leisten können und/oder nur kurzfristig und ohne engere formale Bindung an den Hochschulen tätig sind. Die steigenden Bedarfe der Hochschulen an finanziellen Mitteln werden - auch in der Lehre - mit einem zunehmenden Anteil von Drittmitteln und unter steigender Beteiligung des Bundes gedeckt. Diese Skizze der Hochschulentwicklung seit dem Jahr 2000 bildet den Hintergrund, vor dem die Struktur und Entwicklung der Erziehungswissenschaft als einem der größten universitären Fächer zu betrachten ist. Im Datenreport wird diesem Umstand durch vergleichende Darstellungen einiger Indikatoren der Nachbarfächer Rechnung getragen.

Über diese generellen Strukturveränderungen der Hochschulen hinaus, die sich auch an Kennzahlen der Erziehungswissenschaft ablesen lassen, gibt es im gleichen Zeitraum auch fachspezifische Entwicklungsdynamiken. Zu nennen sind hier die Akademisierung der Ausbildung für pädagogische Berufe etwa im Bereich der frühkindlichen Bildung und die strukturell bedeutsame Forschungsförderung insbesondere seitens des Bundes in den seit 2007 wirksamen Rahmenprogrammen für empirische Bildungsforschung.

1 Alle hier dokumentierten Werte ergeben sich aus Rundungen der Angaben des Statistischen Bundesamts 2019: Studierende: Deutschland, Semester, Nationalität, Geschlecht, Studienfach GENESIS: Tabelle 21311-0003. https://www.destatis.de/DE/Themen/Gesellschaft-Umwelt/ Bildung-Forschung-Kultur/Hochschulen/_inhalt.html;jsessionid=CF261FCE27503C8EEB8 56F43B79B328B.internet712\#sprg233706 [Zugriff: 19.11.2019]. 
Als empirische Grundlage dienen dem Datenreport von Beginn an Kennzahlen, die im Statistischen Bundesamt zu Hochschulen erstellt werden. Deren Quellen sind in erster Linie amtliche Statistiken, die von hoher Aktualität und Zuverlässigkeit sind. Im Laufe seiner Profilierung sind weitere Datenquellen hinzugekommen und für den Datenreport erschlossen worden. $\mathrm{Zu}$ nennen sind die Statistiken des Deutschen Zentrums für Hochschul- und Wissenschaftsforschung (DZHW), Literatur- und Forschungsdatenbanken sowie Dokumentenanalysen von Studienordnungen und Ausschreibungen. Vereinzelt greift der Datenreport auch Ergebnisse von Studien auf, die einen umfassenden Einblick in die Lage des Faches erlauben, so z.B. in diesem Datenreport die Ergebnisse einer Studie zu Stellenausschreibungen für Nachwuchswissenschaftler und Nachwuchswissenschaftlerinnen.

Im Einzelnen berichtet der Datenreport zu folgenden Themen:

Studiengänge und Standorte im Hauptfach: fortschreitende Ausdifferenzierung des Angebots

Nachdem die Umstellung von Diplom- und Magisterstudiengängen auf Bachelor- und Masterstudiengänge abgeschlossen ist, zeigt sich, dass der Strukturwandel an den einzelnen Standorten nicht als abgeschlossen betrachtet werden kann (vgl. Kapitel 1). Vielmehr werden weiterhin dynamisch und in der Breite Studiengänge in relevanter Weise modifiziert, aber auch geschlossen und in neuer Form geöffnet. Die Struktur der Bachelor- und Masterstudiengänge wird in sehr unterschiedlicher Weise zur Profilierung genutzt. Unterschiede zeigen sich sowohl in den Strukturen als auch in den Inhalten. Strukturelle Merkmale bestehen zum Beispiel darin, ob ein Studienprogramm ausschließlich aus der Disziplin Erziehungswissenschaft gespeist wird oder ob ein zweites Studienfach inkludiert ist. Unterschiede zeigen sich auch darin, in welcher Studienphase und in welchem Umfang ein Bachelor- und Masterprogramm stärker disziplinorientiert bzw. stärker beschäftigungsfeldorientiert ausgerichtet ist. Inhaltliche Merkmale betreffen beispielsweise die Ausrichtung an unterschiedlichen Forschungsmethoden oder unterschiedlichen Beschäftigungsfeldern.

Die Ergebnisse verweisen darauf, dass die Bachelor/Master-Struktur vor Ort vielfach kreativ adaptiert wird. So bilden Standorte, an denen auf ein Bachelorprogramm ein Masterprogramm folgt zwar eine relative Mehrheit, aber es finden sich auch Standorte, an denen entweder kein Bachelor oder kein Master bzw. bis zu fünf Bachelor oder bis zu fünf Master angeboten werden. Diese Entwicklung bringt es mit sich, dass unterschiedliche Studienfachbezeichnungen entwickelt wurden, die sich auf Teildisziplinen und Handlungsfelder der Erziehungswissenschaft beziehen (z.B. Kindheitspädagogik, Erwachsenenbildung). 
Hinsichtlich der pragmatischen Ausrichtung werden grundlagenbezogene, forschungsmethodologische, studienrichtungsbezogene und praxisbezogene Schwerpunkte in den Studiengängen unterschieden, aus deren Gewichtung und Kombination sich unterschiedliche Profile für die Studiengänge herausbilden. Insgesamt zeigt sich dabei eine große Ausdifferenzierung erziehungswissenschaftlicher Studiengänge. Wenn davon ausgegangen wird, dass Disziplinen eine Funktion für die Entwicklung von Studiengängen besitzen, so stellt die Heterogenität, die sich aus der Differenzierung ergibt, eine Herausforderung für die Disziplin dar. Es lässt sich die Frage ableiten, wie der Nutzen der Disziplin für die Studiengangentwicklung sowie für die Passung von unterschiedlichen Studienangeboten zueinander in Zukunft entwickelt und gesichert werden kann.

\section{Studiengänge und Standorte der Lehrerinnen- und Lehrerbildung: vielfältige Unterschiede innerhalb und zwischen den Bundesländern}

Ebenso wie die Hauptfachstudiengänge unterlagen auch die Lehramtsstudiengänge in den vergangen zwanzig Jahren einem erheblichen Wandel (vgl. Kapitel 2). Im Unterschied zu den Hauptfachstudiengängen wurde jedoch die Bachelor/Master-Struktur nicht in allen Bundesländern umgesetzt. Zusätzlich gewähren die Länder den Hochschulen zum Teil eine beträchtliche Freiheit hinsichtlich der Strukturierung der Lehramtsstudiengänge, sodass eine Orientierung - nicht nur für Studierende - erschwert ist. Es wird erkennbar, dass das Strukturmodell der DGfE in diesem Zusammenhang, aber auch andere Strukturvorgaben keine Breitenwirkung entfalten konnten, und stattdessen standortspezifische Ressourcen und Vorstellungen die Gestaltung bestimmen. Unterscheidungsmerkmale zwischen den Standorten ergeben sich neben der grundsätzlichen Strukturierung als Staatsexamensstudiengang im Gegensatz zur Bachelor/Master-Variante aus der Länge von Studienprogrammen für einzelne Lehrämter, aus dem Umfang des bildungswissenschaftlichen Anteils sowie von Praxisphasen und schließlich aus dem Ausmaß an Polyvalenz.

An nahezu allen Hochschulen wurden Zentren für Lehrerbildung oder Professional Schools gegründet. Vereinzelt lässt sich auch die Ausbildung von standortübergreifenden Zentren beobachten. Den Zentren werden neben der Verwaltung des Studienbetriebs und der Koordination der Fächer z.T. weitere Aufgaben zugeschrieben. Diese bestehen in der Studiengangentwicklung, der Qualitätssicherung und der Forschung im Bereich von Lehrerbildung, Schulund Unterrichtsentwicklung.

Vor dem Hintergrund der Bedarfsprognose für neu einzustellende Lehrkräfte wird deutlich, dass die vorhandenen Lehramtsstudiengänge an deutschen Hochschulen den Bedarf kurz- und mittelfristig nicht decken können. Um hier Abhilfe zu schaffen, haben die Länder unterschiedliche Qualifizierungsprogramme aufgelegt, die z.T. auf die lokal entwickelten Strukturen zurückgreifen 
und diese für die Ausbildung von Quer- und Seiteneinsteigern nutzen. Eine offene Frage ist, inwiefern diese Entwicklung sich auch auf die Ausgestaltung des bildungswissenschaftlichen Anteils an den Universitäten auswirkt. Vor dem Hintergrund der Eigendynamik innerhalb der Bundesländer und an den Standorten erscheint der Wunsch einer bundeslandübergreifenden Entwicklung jedoch als eine Hoffnung ohne sicheren Grund.

\section{Studienabschlüsse und Verbleib der Absolventinnen und Absolventen}

Daten des Statistischen Bundesamts zu den Studienabschlüssen belegen die Attraktivität und Größe der Erziehungswissenschaft im Fächerspektrum der deutschen Hochschullandschaft (vgl. Kapitel 3). Die Transformation der traditionellen Studiengänge in die Bachelor/Master-Struktur wird hinsichtlich der erzielten Abschlüsse von der Frage begleitet, welcher Anteil der Studierenden unter den veränderten Bedingungen einen Studienabschluss erwirbt, der dem früheren Diplom oder Magister entspricht. Hier zeigen sich für die Erziehungswissenschaft und für die Nachbardisziplinen recht unterschiedliche Entwicklungen. Während in der Erziehungswissenschaft etwas über die Hälfte der Bachelorabsolventen auch einen Masterabschluss erwirbt, liegt diese Quote bei den sozialwissenschaftlichen Nachbardisziplinen um 26 bis $40 \%$ höher.

Die Auswertungen zum Verbleib von Studienabsolventinnen und -absolventen basieren auf repräsentativen Absolventenbefragungen des Deutschen Instituts für Hochschul- und Wissenschaftsforschung (DZHW; vgl. Kapitel 3). Dabei werden Absolventen der Erziehungswissenschaft mit solchen anderer Sozialwissenschaften verglichen. Als erster Übergang interessiert der vom Bachelor in die Masterstudiengänge. Die Ergebnisse der Hochschulstatistik finden hier eine Bestätigung und können vertiefend analysiert werden. Gegen die Aufnahme eines Masterstudiums sprechen aus der Sicht von erziehungswissenschaftlichen Bachelorabsolventinnen und -absolventen weit überdurchschnittlich häufig folgende drei Gründe: der Wunsch, Berufserfahrung zu sammeln, eine unzureichende Finanzierung, das Fehlen eines passenden Studiengangs. Dabei ist der zuletzt benannte Grund besonders auffällig, weil er durch den größten Abstand von erziehungswissenschaftlichen Absolventen zur Gesamtheit der Universitätsabsolventen gekennzeichnet ist.

Als zweiter Übergang fokussiert das Kapitel den Übergang in den Beruf; es zeigt sich zunächst, dass der Wechsel in eine Erwerbstätigkeit für die Absolventen mit Bachelor- und Masterabschlüssen jeweils besser gelingt als im Mittel für alle Hochschulabsolventen. Betrachtet man die Beschäftigung nach fünf und zehn Beschäftigungsjahren, so zeigt sich weiterhin, dass der Übergang in eine vertraglich abgesicherte Beschäftigung im vergangenen Jahrzehnt zunehmend schneller gelungen ist; auch im Vergleich der Fächer stehen Erziehungswissenschaftler hinsichtlich dieses Merkmals gut da. 
Jedoch sehen sich nur zwei Drittel der Absolventen nach einem Jahr als adäquat entsprechend ihres Studiums beschäftigt an. Dies sind $10 \%$ weniger als im Mittel der Universitätsabsolventen. Auch nach fünf und zehn Jahren Berufserfahrung hinkt die Beschäftigungsadäquanz von Absolventinnen und Absolventen der Erziehungswissenschaft zum Teil hinter denen aus vergleichbaren Studiengängen her. Studiengänge können durch die Organisation von Praxiskontakten eine adäquate Beschäftigung auf der lokalen Ebene fördern. Unterdurchschnittlich im Vergleich zu den übrigen Sozialwissenschaften gestaltet sich auch das Einkommen von Absolventinnen und Absolventen der Erziehungswissenschaft, wohingegen die allgemeine Berufszufriedenheit (Arbeitsinhalte, Arbeitszeit, Familienfreundlichkeit) als zunehmend hoch bewertet wird.

\section{Wissenschaftliche Qualifikationen nach dem Studienabschluss in der Erziehungswissenschaft}

Das Kapitel zum Verbleib nach dem Studienabschluss (vgl. Kapitel 3) beschreibt auch den Übergang in ein Promotionsstudium. Hier ist im vergangenen Jahrzehnt zwar ein Anstieg zu beobachten, sodass mittlerweile ein Sechstel der Masterabsolventen eine Promotion aufnehmen, jedoch liegt der Wert immer noch deutlich unter dem Vergleichswert für die Masterabsolventen anderer Disziplinen. Für die Disziplin gravierender ist, dass bislang kein entsprechender Anstieg des Anteils der abgeschlossenen Promotionen bezogen auf die Gesamtheit der Masterabsolventen vier Jahre zuvor (Promotionsintensität des Faches) erreicht werden konnte.

Eine vertiefte Analyse zu Habilitationen und Promotionen in der Erziehungswissenschaft (vgl. Kapitel 6) kann sich einerseits auf die Hochschulstatistik des Statistischen Bundesamts sowie andererseits auf die freiwilligen Meldungen der erziehungswissenschaftlichen Institute bzw. Fachbereiche stützen. Aus den Daten des Statistischen Bundesamts kann geschlossen werden, dass die Anzahl der Promotionen in der Erziehungswissenschaft zwar 2016 einen Höchstwert erreicht, aber mit geringen Schwankungen im Wesentlichen seit 2005 stagniert. Ebenso wuchs die Zahl der Promotionen je 100 Professuren bis 2010 stark an; seitdem ist sie stetig rückläufig. Auch in Psychologie und Sozialwissenschaften zeigen sich auf einem quantitativ insgesamt höheren Promotionsniveau ähnliche Entwicklungen. Der Frauenanteil der Promovierenden in der Erziehungswissenschaft hat sich dabei bis 2015 dem Frauenanteil bei den Absolventen angenähert und stagniert seitdem bei ca. $70 \%$.

Die freiwilligen Meldungen von Promotionen und Habilitationen an die Zeitschrift für Pädagogik erlauben eine thematische Auswertung. Die zugrunde liegenden Dissertationen wenden sich insbesondere der Bildungsforschung, der Sozialpädagogik, der Berufs- und Wirtschaftspädagogik sowie unterrichts- 
bezogenen wie auch lernspezifischen Fragestellungen zu. Themen der Lehrerausbildung wie auch des Lehrerberufs haben den größten Zuwachs in den vergangenen vier Jahren erzielt. Auch die Zahl der Promotionen im frühkindlichen Bereich ist in den letzten Jahren angewachsen. Jedoch hat sich die Häufigkeit sonderpädagogischer Themen durch die Bearbeitung inklusiver Fragestellungen bislang nicht wesentlich verändert. Bildungshistorisch fokussierte Promotionen nehmen erkennbar ab.

\section{Personal: ungleichmäßiger Ausbau von Beschäftigungsmöglichkeiten}

Die Expansion der Studierendenzahlen in den letzten beiden Jahrzehnten macht sich auch beim Personal bemerkbar. Über alle Stellenkategorien hinweg erfolgt im genannten Zeitraum fast eine Verdopplung des Personals (vgl. Kapitel 4). Jedoch profitieren nicht alle Personalkategorien gleichmäßig vom Stellenausbau. Während die Gruppe der Assistenten und Hochschuldozenten allmählich ausläuft und die Anzahl der Professuren stagniert, ist ein starker Anstieg bei den wissenschaftlichen Mitarbeitern und Lehrkräften für besondere Aufgaben zu beobachten. Dadurch werden zwar zusätzliche Qualifikationsmöglichkeiten (wissenschaftliche Mitarbeiterinnen und Mitarbeiter in Drittmittelfinanzierung) geschaffen, aber auch einer Tendenz der Entkoppelung von Forschung und Lehre Vorschub geleistet. Dabei ist weiterhin zu berücksichtigen, dass gleichzeitig die Anzahl der Lehrbeauftragten und Lehraufträge zurückgefahren wurde, wodurch zusätzlich eine Entkoppelung von Lehre und Praxis gefördert wird.

Insgesamt wird weiterhin kritisiert, dass der Stellenaufwuchs zum Großteil in Form von befristeten Stellen erfolgt ist und überwiegend als Teilzeitbeschäftigung ausgeschrieben wird. Nur $11 \%$ der Stellen jenseits der Professur sind aktuell als Dauerstellen angelegt, und 76 \% der Beschäftigten des sogenannten Mittelbaus arbeiten in Teilzeit, wobei ungeklärt ist, zu welchem Anteil dies dem eigenen Wunsch entspricht. Positiv ist hingegen hervorzuheben, dass die Geschlechterparität in der Erziehungswissenschaft seit 1995 zunehmend umgesetzt wurde. Wenn heute $50 \%$ der erziehungswissenschaftlichen Professuren von Frauen besetzt werden, entspricht dies jedoch noch nicht dem Anteil in der Studierendenschaft und auf Mitarbeiterstellen. Insofern besteht hier ein weiter gehendes Entwicklungspotenzial.

Ein Vergleich der Erziehungswissenschaft mit den anderen Fächern zeigt, dass die Erziehungswissenschaft unterdurchschnittlich erfolgreich war, was den Ausbau des Bestands an Professuren angeht. Während der Stellenbestand in Erziehungswissenschaft und Sozialwissenschaft seit 1995 stagniert, können die Politikwissenschaft und die Psychologie einen Aufwuchs von 30 \% vorweisen. Die Wirtschaftswissenschaften verfügen aktuell sogar über zwei Drittel mehr Professuren als zu Beginn des Beobachtungszeitraums. Mit 4,5 Mitarbei- 
tern je Professur weist das Fach zudem einen relativ hohen Quotienten auf, der etwas über die dienstrechtliche Verantwortungsstruktur und Betreuungsdichte an den Hochschulen aussagt.

\section{Forschungsförderung und Publikationen: Ausweis der Forschungskultur}

Zur Entwicklung der Forschungsförderung in der Erziehungswissenschaft kann unter anderem auf Daten der Hochschulstatistik des Statistischen Bundesamts sowie mit einer größeren disziplinären Unschärfe auf die Daten des Förderatlas der Deutschen Forschungsgemeinschaft zurückgegriffen werden (vgl. Kapitel 5). Als weitere Quellen werden die Forschungsdatenbank SOFIS und die "FIS Bildung Literaturdatenbank" genutzt. Für die letzten beiden Jahrzehnte zeigt sich ein kontinuierlicher Anstieg der Drittmitteleinwerbungen pro Professur sowie der damit verbundenen Mitarbeiter, die aus Drittmitteln finanziert werden. Für das Jahr 2017 werden erstmals im Mittel mehr als 1,5 Mitarbeiter je Professur in der Erziehungswissenschaft aus Drittmitteln finanziert. Ein in etwa vergleichbarer Anstieg zeigt sich auch in den anderen sozialwissenschaftlichen Fächern, wobei jeweils disziplinspezifisch unterschiedliche Möglichkeiten zur Drittmitteleinwerbung zu Unterschieden beitragen. Bei Betrachtung der Verteilung lässt eine SOFIS-Datenbankanalyse für die Erziehungswissenschaft auf folgende unterschiedliche Geldgeber schließen: BMBF (33 \%), DFG (20\%), Stiftungen (15\%), Landesministerien und -einrichtungen (14 \%) für die Jahre 2015-2017. Eine Analyse des DFGFörderatlas weist einen Rückgang der bewilligten DFG-Mittel für die Erziehungswissenschaft aus. Der Befund könnte unter anderem mit einer verstärkten Inanspruchnahme von Professuren durch die Qualitätsoffensive Lehrerbildung begründet sein. Weiterhin fällt es der Disziplin schwer, Verbundvorhaben (Forschergruppen, Sonderforschungsbereiche, Graduiertenkollegs) auf den Weg zu bringen.

Eine systematische Betrachtung der Publikationsleistung der deutschen Erziehungswissenschaft leidet besonders unter einer problematischen Datenbasis. Die Erfassung in der »FIS Bildung Literaturdatenbank« erweist sich von Jahr zu Jahr als zunehmend defizitär. Dies führt zum kontrafaktischen Eindruck einer rückläufigen Publikationspraxis. So wird beispielsweise die steigende Anzahl von englischsprachigen Veröffentlichungen systematisch untererfasst. Eine vertiefende Analyse der Publikationsleistung nach Bundesländern lässt darauf schließen, dass die sich entwickelnde quantitative Stärke einzelner erziehungswissenschaftlicher Standorte in den jeweiligen Ländern sowie subdisziplinäre Schwerpunktbildungen an den Standorten und deren Veränderung einen Effekt auf die Publikationsleistung haben. 
Stellenausschreibungen: Unterschiede und Verschiebungen der inhaltlichen Schwerpunktbildung

Ersatzbedarf und Stellenausschreibungen für Professuren werden wie in früheren Auflagen des Datenreports im Kapitel Personal (vgl. Kapitel 4) analysiert, während zu Stellenausschreibungen für Mitarbeiterstellen des wissenschaftlichen Mittelbaus eine gesonderte Analyse vorliegt (vgl. Kapitel 7).

Betrachtet man den Ersatzbedarf für Professuren in der Erziehungswissenschaft, so erschließt sich für das nächste Jahrzehnt ein Ersatzbedarf von knapp zwei Fünfteln der bestehenden Professuren. Damit liegt der Anteil der freiwerdenden Stellen deutlich niedriger als im vergangenen Jahrzehnt, in denen überdurchschnittlich viele Stellen zur Nachbesetzung ausgeschrieben wurden. $\mathrm{Zu}$ gleich könnte die gestiegene Anzahl der wissenschaftlichen Mitarbeiterinnen und Mitarbeiter dazu beitragen, dass die Anzahl der Bewerbungen pro Stelle steigt.

In Bezug auf professorale Stellenausschreibungen erweist sich die Unterscheidung zwischen Fachhochschulen und Universitäten als Leitdifferenz. Während nur ein Zwanzigstel der erziehungswissenschaftlichen Professuren an Universitäten dem Bereich der Sozialpädagogik/Sozialen Arbeit zuzuordnen sind, steigt dieser Anteil auf ein Fünftel, wenn man alle Hochschulen betrachtet. Erziehungswissenschaftliche Professuren an Universitäten wurden zuletzt zu $22 \%$ im Bereich der empirischen Bildungsforschung/empirischen Schulund Unterrichtsforschung ausgeschrieben. Auf diesen Spitzenplatz folgt die Sonderpädagogik/Inklusionspädagogik mit einem Fünftel der Stellen. Jeweils ein knappes Zehntel weisen die Berufs- und Wirtschaftspädagogik sowie die Didaktik/Schulpädagogik auf. Der Anteil der nicht auf Schule und Ausbildung bezogenen Professuren ist erwartungsgemäß geringer.

Die Analyse der Stellenausschreibung für wissenschaftliche Mitarbeiterinnen und Mitarbeiter beruht auf einer umfassenden Erhebung der Ausschreibungen des Jahres 2017. Der Fokus wird von Erziehungswissenschaft auf Bildungsforschung ausgedehnt, weil entsprechende Stellen bis auf wenige Ausnahmen auch für Absolventinnen und Absolventen erziehungswissenschaftlicher Studiengänge offen sind. Ein wichtiges Ergebnis ist hier, dass $23 \%$ der Ausschreibungen eine Promotion voraussetzten. Zugleich gibt es dreimal mehr Stellen für Absolventen mit Masterabschluss als für Promovierte. Bei der Beurteilung dieses Zahlenverhältnisses und der Chancen, nach der Promotion im Wissenschaftssystem zu verbleiben, wird man berücksichtigen müssen, dass erstens nicht alle Stellen mit der Voraussetzung Masterabschluss auf das Qualifikationsziel Promotion ausgerichtet sind (64\% aller Stellen sind als Promotionsstellen ausgeschrieben), und zweitens nur ein reduzierter Anteil der ausgeschriebenen Stellen mit Promotionsmöglichkeit tatsächlich zu einer Promotion führt. Hinsichtlich des Stellenumfangs ist festzuhalten, dass Stellen, die eine Promotion voraussetzen, im Gegensatz zu Promotionsstellen überwiegend als Vollzeitstellen ausgeschrieben sind. 
Ein Viertel aller Stellenausschreibungen fordert Kenntnisse in sowohl qualitativen als auch quantitativen Methoden. Jedoch darf daraus nicht geschlossen werden, dass beide Methodenprofile gleich stark nachgefragt werden. Der Anteil der Stellenausschreibungen, die quantitative Methoden voraussetzten, liegt für alle Qualifikationsstellen (zur Promotion und mit Promotion) um etwa $20 \%$ höher als der Anteil der Stellenausschreibungen, der qualitative Methoden voraussetzt.

\section{Beschreibung der Erziehungswissenschaft und disziplinäre Verantwortung}

Alle Beschreibungen sozialer Organisationen haben politische Implikationen, denn Organisationen basieren auf Entscheidungen, und was beschrieben wird, kann als die Folge von Entscheidungen interpretiert werden. Das gilt auch für den Datenreport Erziehungswissenschaft, der ein differenziertes Bild der Organisation des Faches im System der deutschen Hochschulen liefert. Die auf empirische Daten gestützte Beschreibung bietet einen Bezugspunkt, von dem ausgehend Bewertungen, Entwicklungsperspektiven und vielleicht sogar Handlungsoptionen zu gewinnen sind, die allerdings bei der Größe, Heterogenität und Komplexität des Faches aus unterschiedlichen Interessenpositionen vorgenommen werden. In diesem Sinne fordert der Datenreport Erziehungswissenschaft die Vertreterinnen und Vertreter des Faches zur Diskussion auf.

Freilich können nicht alle in diesem Band berichteten Aspekte eindeutig auf Verantwortlichkeiten in der Erziehungswissenschaft hin adressiert werden. Vor dem Hintergrund der einleitenden Passagen wird deutlich, dass viele der Entwicklungen im Fach - bspw. der Aufwuchs der Studierendenzahlen und die Verlagerung der relativen Häufigkeiten zwischen den Personalkategorien - einem allgemeinen Trend folgen. Eine hochschul- und fachpolitisch brisante Frage betrifft in diesem Kontext die Ausgestaltung wissenschaftlicher Lehre unter dem Umstand einer zunehmenden Entkopplung des Lehrpersonals sowohl von der Forschung als auch von der Praxis. Vor dieser Frage stehen allerdings auch andere Fächer. In der Erziehungswissenschaft ist sie auch angesichts der für das Fach konstitutiven Praxisbezüge und ihrer Leistungen in der Vorbereitung auf Professionalität zu diskutieren.

Weitere Aspekte liegen in der Verantwortung der Erziehungswissenschaft und ihrer innerfachlichen Organisation. Hervorzuheben ist hier etwa die hohe Diversität in der standortspezifischen Ausgestaltung erziehungswissenschaftlicher Studienangebote. Sie lässt sich nicht nur mit Verweis auf das Spektrum wissenschaftlicher Ansätze erklären, sondern bildet unter anderem auch Differenzierungen bezüglich der Zielsetzungen des Studiums und das fehlende gemeinsame Verständnis eines grundlegenden Kanons ab. Die daran gekoppelten Entscheidungen betreffen das Kerncurriculum, dessen Funktion 
und Bindungswirkung aufgrund der Befundlage in Frage steht, und die innere Strukturierung des Faches in Subdisziplinen einschließlich ihrer Funktion als Bezugsdisziplin(en) für pädagogische Professionen. Letztgenannter Aspekt wäre auch vor dem Hintergrund der Veränderungen der Denominationen von Professuren und des sprunghaften Anstiegs der quantitativen Repräsentation von Themen angesichts politisch initiierter Bildungsreformen (bspw. Inklusion) zu diskutieren. Die formale Ausdifferenzierung des Faches sollte dabei nicht nur hinsichtlich ihrer Funktionalität nach innen - bspw. im Sinne der Spezialisierung von Forschungsmethoden und -gebieten -, sondern auch nach außen - bspw. bei der Abgrenzung zu den bisweilen um Deutungshoheit und Ressourcen konkurrierenden Nachbarfächern - diskutiert werden. Nicht zuletzt bedingt die Struktur eines Faches maßgeblich die Möglichkeiten, von außen herangetragene Erwartungen - bspw. in der Lehrkräftebildung - unter Wahrung einer wissenschaftlich-disziplinären Logik in Lehr- und Forschungsgebiete zu transformieren. Dieser mit aller Vorsicht als konzeptionell zu bezeichnende Aspekt der disziplinären Struktur steht in Verbindung mit Merkmalen, die sich statistisch beschreiben lassen. So wird an der Personalstatistik der massive Aufwuchs an Personal - insbesondere in Phasen der wissenschaftlichen Qualifizierung - deutlich. In dem Maße, in dem das Fach Verantwortung für die Absolventinnen und Absolventen seiner Studiengänge hinsichtlich ihrer Chancen auf dem Arbeitsmarkt trägt, so trägt es auch Verantwortung für die Anschlussperspektiven der Wissenschaftlerinnen und Wissenschaftler, die eine Promotion oder Professur anstreben. Die vorliegenden Daten zerstreuen jede realistische Erwartung auf eine Fortsetzung der Karrieren aller Promovierenden und Promovierten im Wissenschaftssystem. Vor diesem Hintergrund ist die Promotion nicht ausschließlich als Einstieg für Karrieren in der Wissenschaft, sondern - im Sinne einer weiteren Verwissenschaftlichung der Professionen - auch für Karrieren in den pädagogischen Handlungsfeldern zu betrachten. Daraus ergibt sich der Bedarf, Optionen der Laufbahnentwicklung in den frühen Phasen akademischer Karrieren zu thematisieren.

Die Deutsche Gesellschaft für Erziehungswissenschaft ist das fachwissenschaftliche und fachpolitische Forum für die Diskussion der genannten und weiterer Themen, die sich aus dem Datenreport ergeben. Einige der hier thematisierten Merkmale des Faches kann die DGfE in ihrem eigenen Entscheidungsbereich gestalten. In der gegenwärtigen Situation sind davon die dringlichsten die disziplinäre und subdisziplinäre Struktur der Erziehungswissenschaft mit Blick auf ihre Repräsentanz an Hochschulen und die Kohärenz ihrer Studienangebote sowie die Vorbereitung akademischer Karrierewege der Erziehungswissenschaftlerinnen und Erziehungswissenschaftler. 


\section{Literatur}

Krüger, Heinz-Hermann/Merkens, Hans/Otto, Hans-Uwe/Rauschenbach, Thomas/ Schenk, Barbara/Weishaupt, Horst/Zedler, Peter (2000): Vorbemerkung. In: DGfE: Datenreport Erziehungswissenschaft. Befunde und Materialien zur Lage und Entwicklung des Faches in der Bundesrepublik. Hrsg. v. Otto, H.-U./Krüger, H.-H./ Merkens, H./Rauschenbach, T./Schenk, B./Weishaupt, H./Zedler, P. Schriften der Deutschen Gesellschaft für Erziehungswissenschaft. Opladen: Leske + Budrich, S. 11-13.

Otto, Hans-Uwe/Zedler, Peter (2000): Zur Lage und Entwicklung des Faches Erziehungswissenschaft in Deutschland. Befunde und Konsequenzen des bundesweiten Datenreports. In: DGfE: Datenreport Erziehungswissenschaft. Befunde und Materialien zur Lage und Entwicklung des Faches in der Bundesrepublik. Hrsg. v. Otto, H.-U./Krüger, H.-H./Merkens, H./Rauschenbach, T./Schenk, B./Weishaupt, H./Zedler, P. Schriften der Deutschen Gesellschaft für Erziehungswissenschaft. Opladen: Leske + Budrich, S. 15-25. 


\title{
1 Studiengänge und Standorte im Hauptfach
}

\author{
Cathleen Grunert, Katja Ludwig \& Kilian Hüfner
}

Mit dem Datenreport 2020 liegen nun zum sechsten Mal Daten zu Studiengängen und Standorten im Bereich der erziehungswissenschaftlichen Hauptfachstudiengänge an den deutschen Hochschulen und Universitäten vor. Die gesamtdeutsche Dokumentation vorhandener Studiengänge sowie die Perspektive auf die Veränderungen der letzten Jahre ermöglicht eine vergleichende Betrachtung unterschiedlicher Studienmodelle und Entwicklungen. Zentrale Fragestellung ist, inwiefern sich die durch den Bologna-Prozess intendierten Forderungen nach Standardisierung und Flexibilisierung von Studiengängen sowie eine verbesserte "Employability « ${ }^{1}$ der Absolventinnen und Absolventen zwanzig Jahre nach Beginn der Reform in den Ausbildungsmodellen wiederfinden. Die aktuelle Bestandsaufnahme verweist darauf, dass in den Hauptfachstudiengängen mittlerweile zwar flächendeckend Bachelor- und Masterstudiengänge angeboten werden, dass die einzelnen Hochschulstandorte dies jedoch weiterhin sowohl strukturell als auch inhaltlich sowie bezogen auf den Umfang einzelner Studienelemente auf sehr unterschiedliche Weise tun. Die Längsschnittperspektive zeigt zudem eine große Fluktuation: Studiengänge werden wieder eingestellt, weiterhin neu eingerichtet oder auch entscheidend modifiziert. Der Umstellungsprozess auf die neuen Abschlüsse ist zwar abgeschlossen, aber das Feld ist anscheinend ständig in Bewegung, was sowohl mit Entwicklungen in Wissenschaftsdisziplin und Arbeitsmarkt, aber nicht zuletzt auch mit der Praxis der Reakkreditierung in Verbindung steht.

Der Beitrag nimmt vor diesem Hintergrund zum einen Stand und Veränderungen in der Struktur der Studiengänge etwa hinsichtlich der Studiendauer und der Umfänge in den Blick, und fokussiert zum anderen auf die Profilbildungen der Studienprogramme und die damit verbundenen disziplinären oder teildisziplinären Schwerpunktsetzungen. Und schließlich wird mit Blick auf verschiedene Studieninhalte und deren Stellenwert im Gesamtstudienprogramm die Frage aufgeworfen, inwiefern erziehungswissenschaftliche Studiengänge sich in ihren Ausbildungsinhalten überhaupt auf die Vermittlung allgemein-disziplinären Grundlagenwissens beziehen und wie sie auch über ihre Fachbezeichnungen symbolische Grenzziehungen vornehmen, die auf eine deutliche Ausdifferenzierung des Faches verweisen.

Zur Bearbeitung dieser Fragen werden die erziehungswissenschaftlichen Hauptfachstudiengänge auf Bachelor- und Masterebene an allen Universitä-

1 Vgl. zur Problematik der Übersetzung des Begriffs zwischen »Beschäftigungsfähigkeit«, »Berufsrelevanz« oder »Praxisrelevanz« auch Schubarth und Speck 2014. 
ten und Pädagogischen Hochschulen hinsichtlich ihrer Studienstruktur, ihrer inhaltlichen Ausgestaltung sowie im Hinblick auf unterschiedliche Muster an Studiengängen vorgestellt und analysiert. Ein Schwerpunkt liegt dabei auf der Frage, inwiefern sich die Studiengangsgestaltung an den Empfehlungen des Kerncurriculums Erziehungswissenschaft der DGfE (DGfE 2010) orientiert und welche unterschiedlichen Typen von Studiengängen sich daraufhin nachzeichnen lassen und inwiefern sich diese mit Blick auf die letzten Erhebungen (Grunert et al. 2016; Stisser et al. 2012) verändert haben. Der Beitrag schließt mit einem Fazit, in dem abschließend Trends benannt und mögliche Entwicklungen aufgezeigt werden.

\subsection{Studiengänge und Standorte im Überblick}

Zum Wintersemester 2018/19 finden sich an insgesamt 64 Universitäten und Pädagogischen Hochschulen in Deutschland 194 Bachelor- und Masterstudiengänge, die hauptverantwortlich von erziehungswissenschaftlichen Fakultäten bzw. Instituten getragen werden. ${ }^{2}$ Diese hohe Zahl an Studiengängen kommt vor allem durch die Zweiteilung in Bachelor- und Masterstudiengänge und durch das teilweise parallele Angebot an Ein- und Zwei-Fach-Studiengängen zustande. ${ }^{3}$ Unter den Studiengängen finden sich 86 Bachelor- und 108 Masterstudiengänge. Während die Anzahl der Bachelorstudiengänge im Vergleich zum Wintersemester 2014/15 konstant bleibt, erfährt der Masterbereich einen Aufwuchs um vier Studiengänge und wächst damit weiter an (Grunert et al. 2016: 21).

Mit Blick auf die Standorte zeigt sich, dass aktuell 56 Standorte Bachelorund 62 Standorte Masterstudiengänge anbieten. An 54 Standorten werden sowohl Bachelor- als auch Masterstudiengänge angeboten und zehn Standorte

2 Die nachfolgenden Darstellungen basieren in ihrer Grundstruktur auf Erhebungen im Rahmen des von der DFG geförderten Projekts "Erziehungswissenschaft im Bologna-Prozess" (z.B. Grunert/Ludwig 2016a). Hier wurde u.a. eine Bestandsaufnahme aller in erziehungswissenschaftlicher Verantwortung liegender Hauptfachstudiengänge vorgenommen. Aufgenommen wurden damals Studiengänge, die von erziehungswissenschaftlichen Organisationseinheiten hauptverantwortlich (mit-)getragen wurden und in die zum Wintersemester 2013/14 eine Immatrikulation in das erste Fachsemester möglich war. Als Hauptfach wurde ein Studiengang eingeordnet, wenn es möglich war, die Abschlussarbeit in diesem anzufertigen. Im Falle starker Spezialisierungen wurden alle Studiengänge aufgenommen, die sich an den etablierten Studienrichtungen der Diplomstudiengänge und/oder Teildisziplinen der Erziehungswissenschaft orientieren (vgl. auch schon Stisser et al. 2012). Dieses Vorgehen wurde für den Datenreport 2016 wiederholt und bestimmt nun auch die hier dargestellte aktuelle Datenbasis von Studiengängen zum Wintersemester 2018/19.

3 Zudem bieten manche Standorte Zwei-Fach-Studiengänge in unterschiedlichen Umfängen an, die hier auch entsprechend mehrfach gezählt wurden. An einigen Standorten werden, der Eigendefinition nach, auch Ein-Fach- bzw. Zwei-Fach-Studiengänge angeboten, die jedoch unterschiedliche Strukturvarianten ermöglichen, sodass auch in diesen Fällen die Studiengänge mehrfach gezählt wurden (vgl. Anhang Tabelle A1 und A2). 
halten entweder nur Bachelor- oder nur Masterstudiengänge vor (2:8). Im Vergleich zur Situation vor der Studienreform ist es damit nach wie vor an 10 Standorten nicht mehr möglich, eine zumindest in zeitlicher Hinsicht vergleichbare Langzeitausbildung im Feld der Erziehungswissenschaft zu absolvieren. Von den ehemals 66 Standorten (vgl. Grunert 2012; HRK 1998) bieten nur noch 54 einen erziehungswissenschaftlichen Studiengang als Bachelor- und Mastervariante im Hauptfach an. Gleichzeitig werden jedoch von sieben Standorten Bachelor- und/oder Masterstudiengänge im Hauptfach bereitgehalten, die vormals keinen Diplom- oder Magisterhauptfachstudiengang im Studienangebot hatten, sodass der Abbau dadurch auch wieder kompensiert werden konnte.

Abbildung 1.1: Anzahl der Bachelor- und Masterstudiengangskombinationen an den Standorten ( $\mathrm{n}=64$; in absoluten Häufigkeiten)

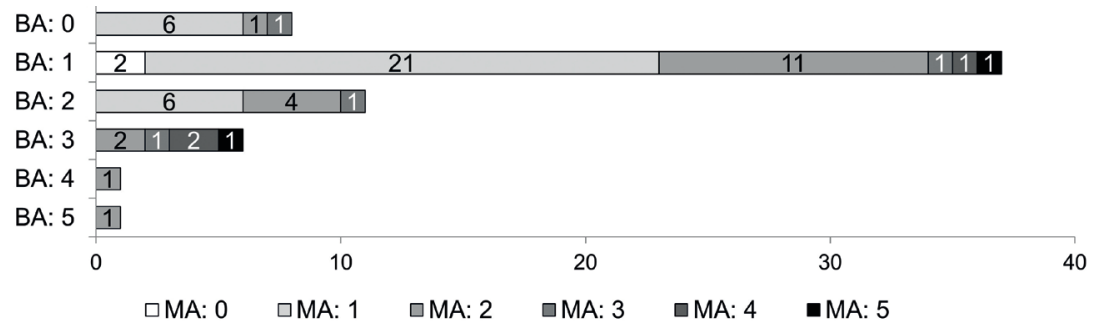

Im Durchschnitt haben die erziehungswissenschaftlichen Studienstandorte drei Studiengänge im Angebot, wobei die standortbezogene Anzahl von einem bis acht Studiengängen reicht (vgl. Abbildung 1.1). Am häufigsten finden sich weiterhin Standorte, die einen Bachelor- mit einem Masterstudiengang verbinden $(n=21,33 \%)$, gefolgt von der Kombination eines Bachelorstudiengangs mit zwei Masterangeboten ( $\mathrm{n}=11,17 \%)$. Sechs Standorte $(9 \%)$ bieten zwei Bachelor- und einen Masterstudiengang an. Während mit drei Bachelorund fünf Masterstudiengängen die PH Ludwigsburg die meisten Ein-FachStudiengänge vorhält, gefolgt von der PH Weingarten mit sieben Ein-FachStudiengängen, ist bei einer Reihe der Standorte die hohe Anzahl an Studiengängen vor dem Hintergrund von Ein- und Zwei-Fach-Varianten im Bachelorbereich einzuordnen.

\subsubsection{Struktur der Studiengänge}

Dabei hat sich im Vergleich zur letzten Erhebung (Grunert et al. 2016:22) auf der Masterebene das Modell der Ein-Fach-Studiengänge noch deutlicher durchgesetzt (102:6), während sich im Bachelorbereich weiterhin nahezu ähnlich häu- 
fig Ein-Fach- und Zwei-Fach-Studiengänge (45:41) finden. An Standorten mit Bachelor- und Masterangeboten gibt es am häufigsten die Kombination von mindestens einem Ein-Fach-Bachelor- und einem Ein-Fach-Masterstudiengang $(n=33)$. Diese Variante ist mehrheitlich an ehemaligen Diplomstandorten $(\mathrm{n}=21) \mathrm{zu}$ finden. Ebenfalls häufig ist die Kombination mindestens eines ZweiFach-Bachelors mit einem Ein-Fach-Master $(n=20)$. Demgegenüber ist es eher unüblich, ausschließlich Zwei-Fach-Varianten im Bachelor- und Masterbereich anzubieten, sodass sich dieses Modell auch nur an einem Standort findet. Bei Standorten, die nur Bachelor- oder Masterstudiengänge anbieten $(\mathrm{n}=10)$, dominiert das Ein-Fach-Modell. Jedoch bieten zwei Standorte ihren Bachelorstudiengang ausschließlich in der Zwei-Fach-Variante an.

Die Gesamtumfänge der erhobenen Studiengänge liegen unverändert im Bachelorbereich zwischen 70 und 210 sowie im Masterbereich zwischen 69 und 120 Leistungspunkten. ${ }^{4}$ Vor allem auf der Bachelorebene, auf der Ein-Fachund Zwei-Fach-Studiengänge fast paritätisch verteilt sind, lassen sich entsprechend große Differenzen in den Umfängen ausmachen, sodass insgesamt 14 verschiedene Werte dokumentiert werden können, die sich bei 90 (ca. $14 \%$ ) und 120 (ca. $10 \%$ ) ansatzweise und bei 180 (ca. $57 \%$ ) Leistungspunkten deutlich konzentrieren. Im Bereich der Ein-Fach-Struktur finden sich erneut zwei Studiengänge, die vom sechssemestrigen 180 LP-Modell abweichen, indem sie längere Praktikumszeiten in ihre Programme integrieren und diese als siebensemestrige Bachelorstudiengänge mit $210 \mathrm{LP}$ auslegen.

Im Master, der auch vorwiegend als Ein-Fach-Studiengang angeboten wird, dienen die 120 Leistungspunkte hingegen als übergreifende Richtgröße und werden nur von etwa $10 \%$ der Studiengänge unterboten, wobei sich hier weiterhin vier Studiengänge finden, die als Ein-Fach-Studiengänge mit 90 LP (3 Semester) ausgelegt sind und entsprechend ein Bachelorstudium mit 210 LP (7 Semester) voraussetzen.

\subsubsection{Veränderungen im Vergleich zum Wintersemester 2014/15}

Schaut man sich die Veränderungen im Vergleich zu den letzten Erhebungen an, dann ist, wenn auch etwas moderater, weiterhin Bewegung in der erziehungswissenschaftlichen Studiengangslandschaft. Waren es zwischen dem Wintersemester 2011/12 (Stisser et al. 2012) und 2014/15 (Grunert et al. 2016) noch 15 Masterstudiengänge und 4 Bachelorstudiengänge, die neu eingeführt wurden, so sind es zwischen dem Wintersemester 2014/15 und 2018/19 acht

Im Fall von Zwei-Fach-Studiengängen, bei denen die Abschlussarbeit optional in einem der beiden Fächer angefertigt werden kann $\left(n_{B A}=15 ; n_{M A}=4\right)$, wurden die Umfänge für die Abschlussarbeit in den Gesamtumfang eingerechnet, und auch verpflichtende Praktika, die außerhalb der jeweiligen Fächer in einem Optionalbereich aufgehoben sind, wurden in diesen einbezogen $\left(\mathrm{n}_{\mathrm{BA}}=10\right)$. 
Masterstudiengänge, die neu auf das Tableau kommen. ${ }^{5}$ Auf der Ebene der Bachelorstudiengänge scheint sich damit das Gefüge der Studiengänge eher zu konsolidieren. Auf der Masterebene wurden überwiegend bereits bestehende Masterangebote an den jeweiligen Standorten durch zusätzliche Studiengänge ergänzt. Nur an einem Standort wurde erstmals überhaupt ein Masterstudiengang eingeführt.

Somit blicken wir zwar äußerlich auf eine Konsolidierung der Studiengangsangebote insgesamt, jedoch lassen sich im Vergleich zur letzten Erhebung weiterhin deutliche Bewegungen innerhalb der Studiengänge, also bezogen auf ihre inhaltliche und strukturelle Ausrichtung, feststellen. Nur bei $47 \%$ der Studiengänge wurden keine oder nur sehr leichte Veränderungen vorgenommen $(\mathrm{n}=85)$, während 7 Studiengänge ihre Fachbezeichnung geändert haben und immerhin an 53 \% der Studiengänge $(\mathrm{n}=96)$ relevante Änderungen an den Studienstrukturen und/oder Inhalten ausgemacht werden konnten. Zudem wurden zwei BA-Studiengänge und vier MA-Studiengänge im erwähnten Zeitraum wieder abgeschafft. ${ }^{\circ}$

\subsection{Studienfachbezeichnungen}

Auf der Ebene der Konzeption als Ein-Fach- oder Zwei-Fach-Varianten und auch hinsichtlich ihrer Umfänge sind die Bachelorstudiengänge weiterhin deutlich heterogener als die Masterstudiengänge. Allerdings sind anhand der Strukturmerkmale noch keine Aussagen über inhaltliche Aspekte der erziehungswissenschaftlichen Hauptfachstudiengänge möglich. Um sich diesen anzunähern, wird im Folgenden zunächst ein Blick auf die Studienfachbezeichnungen geworfen, die einen ersten Hinweis auf mögliche Profilbildungen in den einzelnen Studienprogrammen geben können. Dabei bleibt - trotz einiger Änderungen - die bereits in den letzten Erhebungen festgestellte Heterogenität der Studienfachbezeichnungen erhalten, die über die strukturelle auch auf eine inhaltliche Pluralität der Studiengangslandschaft im Feld der erziehungswis-

5 Zwei Studiengänge wurden in der aktuellen Erhebung mit aufgenommen, die es anscheinend zum letzten Erhebungszeitpunkt auch bereits gegeben hat, die jedoch über unser Suchraster nicht in den Blick geraten sind. Diese sind der Bachelorstudiengang "Mehrsprachigkeit und Interkulturelle Bildung « an der PH Weingarten sowie der Zwei-Fach-Bachelor »Erziehungswissenschaft « als Teil des Studiengangs »Kultur und Gesellschaft» der Universität Bayreuth.

6 Dies sind der Masterstudiengang "Bildungs- und Wissensmanagement « der TH Aachen, der MA-Studiengang »Theaterpädagogik « der Universität Erlangen-Nürnberg, der Zwei-FachMasterstudiengang »Erziehungswissenschaft mit dem Schwerpunkt Forschung und Entwicklung im Bildungswesen « der Universität Göttingen, der durch einen Ein-Fach-Master ersetzt wurde, der Zwei-Fach-BA »Erziehungswissenschaft" der Uni Münster, wo es BA und MA jetzt nur noch in der Ein-Fach-Variante gibt, sowie der BA-Studiengang »Pädagogik « der KU Eichstätt-Ingolstadt, der aber ab Wintersemester 2019/20 durch den Studiengang »Bildungsund Erziehungswissenschaft« ersetzt wird. 
senschaftlichen Hauptfachstudiengänge verweist. Wie in der letzten Erhebung (Grunert et al. 2016: 23) finden sich bei nun 194 Studiengängen ganze 90 unterschiedliche Studienfachbezeichnungen. Dabei sind es erneut die Zwei-FachStudiengänge, die mehrheitlich generalisierte Fachbezeichnungen tragen.

\subsubsection{Generalisierte Studienfachbezeichnungen}

Etwas weniger als die Hälfte (45\%) bzw. 88 der Studiengänge $\left(\mathrm{n}_{\mathrm{BA}}=52\right.$; $\left.\mathrm{n}_{\mathrm{MA}}=36\right)$ tragen die Bezeichnung »Erziehungswissenschaft(en)«, »Bildungswissenschaft(en)«, teilweise in Ergänzung von "Educational Science(s)« oder "Pädagogik« bzw. Kombinationen aus diesen Begriffen und werden im Folgenden als generalisierte Studiengänge bezeichnet, wohlwissend, dass eine solche Zuordnung zunächst nur auf dem Labeling und nicht auf den tatsächlich integrierten Studieninhalten beruht (vgl. Abbildung 1.2).

Zwar findet sich in dieser Gruppe der Disziplinbegriff »Erziehungswissenschaft « als alleiniger und im Singular, wie ihn auch die Fachgesellschaft DGfE verwendet, am häufigsten $\left(\mathrm{n}_{\mathrm{BA}}=26 ; \mathrm{n}_{\mathrm{MA}}=14\right)$, jedoch wird dieser zum einen an manchen Standorten auch im Plural $\left(\mathrm{n}_{\mathrm{BA}}=3 ; \mathrm{n}_{\mathrm{MA}}=1\right)$ verwendet. Zum anderen werden als generalisierte Studienfachbezeichnungen über alle generalisierten Kategorien hinweg auch die Begriffe "Pädagogik» $\left(\mathrm{n}_{\mathrm{BA}}=13 ; \mathrm{n}_{\mathrm{MA}}=5\right)$ oder »Bildungswissenschaft $(\mathrm{en}) \ll\left(\mathrm{n}_{\mathrm{BA}}=11 ; \mathrm{n}_{\mathrm{MA}}=16\right)$ gebraucht.

Abbildung 1.2: Studienfachbezeichnungen $\left(\mathrm{n}_{\mathrm{BA}}=86 ; \mathrm{n}_{\mathrm{MA}}=108\right.$; in $\%$ und (n))

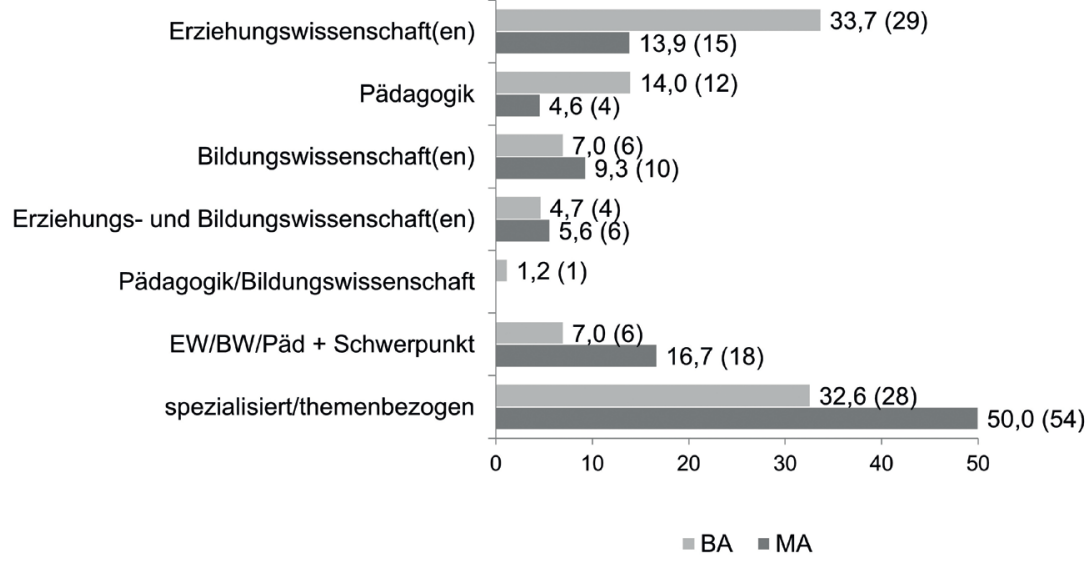

Veränderungen im Vergleich zur letzten Erhebung sind hier eher marginal, wenngleich generalisierte Fachbezeichnungen im Master etwas zugenommen 
haben (vgl. Grunert et al. 2016: 24). Während »Pädagogik« als Studienfachbezeichnung im Masterbereich weiterhin deutlich seltener vorkommt, ist hier dennoch ein Studiengang mit dieser Bezeichnung hinzugekommen, allerdings nur aufgrund der Streichung des Schwerpunktzusatzes, gleichzeitig ist im Bachelorbereich einer entfallen. ${ }^{7}$ Der Begriff »Bildungswissenschaft(en)« hat aktuell zwar jeweils einen Zuwachs von einem Studiengang, jedoch kommt dies im BA nur durch den Wegfall eines Schwerpunktzusatzes zustande, im MA geht dies auf die Neueinführung an der PH Heidelberg zurück. Die Begriffsverwendung "Bildungswissenschaft" für die Bezeichnung von Hauptfachstudiengängen ist ohnehin sehr häufig an pädagogischen Hochschulen zu finden $(n=5)$, wenngleich der Begriff ebenso zur Kennzeichnung der nicht-fachbezogenen Studienanteile in der Lehrerbildung verwendet wird.

Insgesamt 12 \% bzw. 24 weitere Studiengänge ergänzen zudem diese generalisierten Bezeichnungen durch spezifische Schwerpunkte, wobei sich diese Art des Labelings deutlich häufiger im Master- als im Bachelorbereich findet $\left(\mathrm{n}_{\mathrm{BA}}=6 ; \mathrm{n}_{\mathrm{MA}}=18\right)$. Auch hier wird am häufigsten "Erziehungswissenschaft" $\left(\mathrm{n}_{\mathrm{BA}}=3 ; \mathrm{n}_{\mathrm{MA}}=12\right)$, gefolgt von »Bildungswissenschaft(en $)_{\ll}\left(\mathrm{n}_{\mathrm{BA}}=2 ; \mathrm{n}_{\mathrm{MA}}=5\right)$ und "Pädagogik" $\left(\mathrm{n}_{\mathrm{BA}}=1 ; \mathrm{n}_{\mathrm{MA}}=1\right)$ als übergreifende Bezeichnung verwendet und durch Schwerpunktthemen ergänzt. Fünf der Schwerpunkte verweisen dabei eher auf allgemeine, querliegende Themen (z.B. »Erziehung und Bildung in gesellschaftlicher Heterogenität» oder »Bildungstheorie und Gesellschaftsanalyse«) und können damit in die Gruppe der generalisierten Studienfachbezeichnungen eingeordnet werden $\left(\mathrm{n}_{\mathrm{BA}}=1 ; \mathrm{n}_{\mathrm{MA}}=4\right)$. Die überwiegende Mehrheit der Studiengänge $\left(\mathrm{n}_{\mathrm{BA}}=5 ; \mathrm{n}_{\mathrm{MA}}=14\right)$ verweist hier jedoch entweder auf spezifische Themenbereiche (z.B. »Organisationsentwicklung «, »Forschung und Entwicklung in Organisationen") oder teildisziplinäre Schwerpunktsetzungen (z.B. »Sozialpädagogik«, »Pädagogik der Kindheit«). ${ }^{8}$ Im Vergleich zur letzten Erhebung (vgl. Grunert et al. 2016: 253ff.) ist in der Gruppe der Studiengänge, die einen generalisierten Begriff mit einem Schwerpunktzusatz verbinden im BA-Bereich sowie im MA-Bereich jeweils einer dazugekommen, der seiner ehemals spezialisierten Bezeichnung nun den Begriff Erziehungswissenschaft vorangestellt hat (Gießen, Flensburg), bei zwei Studiengängen wurde der Begriff Erziehungswissenschaft nun durch einen Schwerpunkt ergänzt (Tübingen, Freiburg).

Von den acht im Vergleich zum letzten Datenreport 2016 neu eingeführten Studiengängen kann nur einer der Gruppe der generalisierten Studienfachbezeichnungen zugeordnet werden, alle anderen fallen unter die Kategorie spezialisiert, die im Folgenden näher beleuchtet werden sollen.

7 Dieser wird jedoch, wie erwähnt, zum Wintersemester an der KU Eichstätt-Ingolstadt durch den Studiengang "Bildungs- und Erziehungswissenschaft « ersetzt.

8 Diese Studiengänge haben wir auch für die weiteren Betrachtungen der Gruppe der spezialisierten Studiengänge zugeordnet. 


\subsubsection{Spezialisierte Studienfachbezeichnungen}

Sowohl im Bachelor- (42\%) als auch im Masterbereich (82\%) finden sich auch solche Studiengänge, die in ihrem Labeling keinen Bezug auf einen der drei generalisierten Begriffe nehmen, sondern sowohl auf teildisziplinäre als auch auf eher themenbezogene Studiengänge verweisen. Zwei Studiengänge aus dieser Gruppe wurden im Vergleich zum Wintersemester 2014/15 eingestellt, jedoch sind sieben, ausschließlich Masterstudiengänge, neu hinzugekommen, die sich in unterschiedlichen Themen- und pädagogischen Handlungsfeldern bewegen. Rechnet man zu dieser Gruppe diejenigen Studiengänge hinzu, die einen generalisierten Begriff mit einer spezifischen Schwerpunktsetzung verbinden, dann ergeben sich 101 Studiengänge mit spezialisierten Studienfachbezeichnungen. Dieses Feld ist im Hinblick auf die verwendeten Fachbezeichnungen zugleich so heterogen, dass sich immerhin 73 der insgesamt 90 unterschiedlichen Studienfachbezeichnungen in dieser Gruppe finden, wobei gleiche Fachbezeichnungen oft nur aufgrund der Bachelor- und Mastervariante des jeweiligen Studiengangs am selben Standort zustandekommen. Dies ist elfmal der Fall, sodass fast jeder Standort seine spezialisierten Studiengänge mit einem unikaten Label versieht (!) - womit hier auch eher die Frage nach Alleinstellungsmerkmalen und Profilbildungen im Vordergrund zu stehen scheint, als die Frage nach teildisziplinärer Verselbstständigung.

Abbildung 1.3: spezialisierte Studienfachbezeichnungen $\left(\mathrm{n}_{\mathrm{BA}}=33 ; \mathrm{n}_{\mathrm{MA}}=68\right.$; in $\%$ und $(n))$

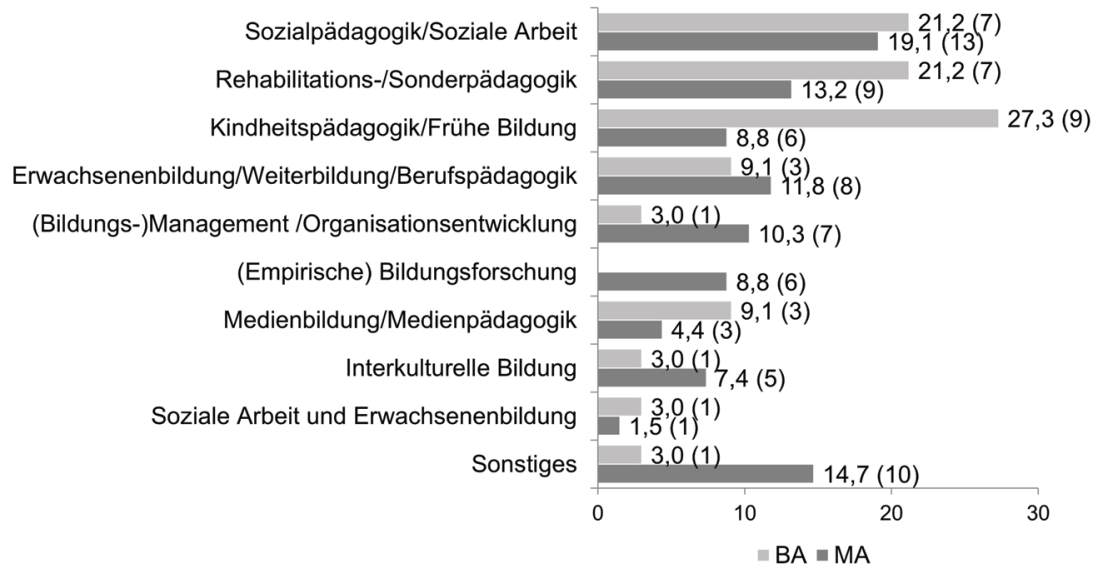


Etwa die Hälfte der Studiengänge lassen sich dabei der Sozialpädagogik, Rehabilitationspädagogik und Erwachsenenbildung zuordnen (vgl. Abbildung 1.3), die auch schon vor der Bologna-Reform die zentralen Studienrichtungen waren und sich in eigenen Studiengängen institutionalisiert haben.

Gleichzeitig findet sich - wie bereits erwähnt - auch weiterhin innerhalb dieser etablierten Teildisziplinen eine ausgeprägte Pluralität im StudienfachLabeling, die durch die vorgenommene Gruppierung verdeckt wird. So finden sich bei 20 Studiengängen im Bereich Sozialpädagogik/Soziale Arbeit allein 13, bei den 11 Studiengängen im Bereich der Erwachsenenbildung/Weiterbildung 7 und bei den 16 Studiengängen im Bereich der Rehabilitations-/ Sonderpädagogik 5 unterschiedliche Fachbezeichnungen. Damit ist der Bereich der Rehabilitations-/Sonderpädagogik die am konstantesten bezeichnete Gruppe dieses Feldes.

Insbesondere das Feld der Kindheitspädagogik bzw. frühen Bildung hat sich mit 9 Bachelor- und 6 Masterstudiengängen weiter konsolidiert und macht mehr als ein Viertel der spezialisierten Bachelorstudiengänge aus. Im Masterbereich konnte dieser Schwerpunkt weitere zwei Studiengänge hinzugewinnen, sodass es nun auch für die zweite Studienphase ein breiteres Angebot an Studiengängen gibt. Weiterhin werden diese Studiengänge vorrangig von den Pädagogischen Hochschulen angeboten $(n=9)$. Obwohl zwei Studiengänge von »Frühe Bildung «bzw. »Bildung und Förderung in der Kindheit « in »Kindheitspädagogik « umbenannt wurden, sind auch in diesem Bereich die Fachbezeichnungen deutlich different. Das Feld der Medienpädagogik oder Medienbildung konnte trotz der weitreichenden und sich verstärkenden Debatte um die Digitalisierung des gesellschaftlichen Lebens in den letzten Jahren keine - nach außen hin sichtbaren - Zuwächse im Bachelorbereich und nur einen weiteren Studiengang im Master für sich verbuchen.

Unverändert bleibt der Befund, dass die spezialisierten Fachbezeichnungen im Masterbereich gegenüber den Bachelorstudiengängen ein größeres Themenspektrum aufweisen (vgl. Grunert et al. 2016: 26). Hier führen zwar ebenso die klassischen Teildisziplinen die Rangliste an, werden aber gefolgt von dem Bereich (Bildungs-)Management und Organisationsentwicklung, dem der Kindheitspädagogik und der (empirischen) Bildungsforschung, die im Bachelorbereich weiterhin keine Rolle spielt, allerdings auch auf Masterebene zwei Studiengänge weniger als zur letzten Erhebung für sich verbuchen kann (ebd.). Spezialisierungen im Master sind damit nicht nur an etablierte Teildisziplinen gebunden, sondern fokussieren teilweise auf spezifische Handlungsformen (z.B. Organisation, Beratung, Forschung). Und auch Studienfachbezeichnungen, die sich keiner Studienrichtung zuordnen lassen und eher auf bestimmte Themen setzen (z.B. »Bildung in Europa«) oder auf sehr spezielle Handlungsfelder abzielen (z.B. »Abenteuer- und Erlebnispädagogik«), sind deutlich häufiger bei den Masterstudiengängen zu beobachten. 


\subsubsection{Standorte und Studienfachbezeichnungen}

Schaut man schließlich nochmals auf die Standortebene und die dort vorhandenen Kombinationen generalisierter und spezialisierter Studiengänge, zeigt sich, dass an nur knapp der Hälfte der Standorte mindestens ein generalisierter Bachelor- und ein generalisierter Masterstudiengang angeboten wird $(\mathrm{n}=29)$, wovon 16 Standorte ausschließlich diese Kombination anbieten, während die anderen 13 zusätzlich noch spezialisierte Bachelor- oder Masterstudiengänge vorhalten. An sieben weiteren Standorten wird ein generalisierter Bachelor mit einem spezialisierten Master verbunden. Dass nur der Masterstudiengang generalisiert ist und die Bachelorstudiengänge spezialisiert, kommt an fünf Standorten vor. An sechs Standorten wird ein generalisierter Bachelor- durch spezialisierte Bachelor- und Masterstudiengänge ergänzt. Immerhin sieben Standorte verzichten mittlerweile gänzlich auf einen Studiengang mit generalisiertem Labeling und bieten damit ausschließlich spezialisierte Bachelor- und Masterstudiengänge an, während fünf Standorte nur einen spezialisierten Bachelor- oder Masterstudiengang vorhalten.

Im Vergleich zum Wintersemester 2014/15 lässt sich festhalten, dass das hohe Maß der Ausdifferenzierung auf der Labelebene konstant erhalten bleibt und zumindest keine neuen thematischen Bezüge auf der Ebene der Fachbezeichnungen ihren Ausdruck finden. Marginal haben generalisierte Fachbezeichnungen bzw. Verweise auf die Bezugsdisziplin an Bedeutung gewonnen.

Zusammenfassend kann festgehalten werden, dass aktuell insgesamt $45 \%$ der angebotenen Studiengänge ausschließlich generalisierte und $43 \%$ ausschließlich spezialisierte Fachbezeichnungen tragen. 12 \% ergänzen generalisierte Begriffe mit spezifischen Schwerpunkten. Dabei finden sich spezialisierte Studiengänge deutlich häufiger auf der Masterebene (30 zu 70 \%), während sich das Verhältnis bei den Bachelorstudiengängen mit $65 \mathrm{zu} 35 \%$ annähernd umgekehrt darstellt. In struktureller Hinsicht (vgl. Kapitel 1.1) sind es hingegen eher die Bachelor- als die Masterstudiengänge, die sich deutlich heterogener darstellen. Zumindest dem Verhältnis von generalisierten bzw. spezialisierten Bachelor- und Masterstudiengängen nach könnte auch vermutet werden, dass im Bachelorbereich tendenziell breiter in die Disziplin eingeführt wird und darauf aufbauend eine stärkere Schwerpunktsetzung im Master erfolgt. Inwieweit sich dies jedoch auch an inhaltlichen Kriterien widerspiegelt, wird nachfolgend im Zentrum der Betrachtung stehen.

\subsection{Studieninhalte}

Für die nachfolgenden Darstellungen wurde in Anlehnung an das Kerncurriculum Erziehungswissenschaft (KCE) ${ }^{9}$ (vgl. DGfE 2010) und auf der Basis

9 Wenngleich die Vorschläge des Kerncurriculums (KCE) nicht als verbindliche Bezugsgröße für die Einrichtung von Studienprogrammen verstanden werden können, stehen sie als 
einer quantitativen Inhaltsanalyse eine Erhebung der fachbezogenen und berufspraktischen Anteile (Praktika) der Studienprogramme vorgenommen. ${ }^{10}$ Zur Erhebung der fachbezogenen Studieninhalte haben wir uns auf die Anteile an Grundlagen (bezogen auf die Studieneinheit 1 im KCE für BA-Studiengänge: Grundbegriffe, Theorien, Geschichte und wissenschaftstheoretische Ansätze) sowie die Anteile an Forschungsmethoden und Lehrforschungsprojekte (für den Master empfohlen) konzentriert. Mit Blick auf strukturelle Empfehlungen für einen möglichen Umfang der Anteile bleibt das KCE jedoch sehr vage, da diese nur integriert neben anderen Studieneinheiten ${ }^{11}$ aus einem Pflicht- und einem Wahlbereich mit jeweils 26 Leistungspunkten (LP) ausgewiesen werden. Geht man jedoch hypothetisch von einer symmetrischen Verteilung der LP im Pflichtbereich aus, dann sollten für den Bereich der Grundlagen wenigstens 9 LP und für den Bereich der Forschungsmethoden mindestens 3 LP im BA und im MA zusätzlich 1 bis 2 LP für Lehrforschungsprojekte vorgesehen werden.

Darüber hinaus haben wir die (wahl-)verpflichtenden studienrichtungsbezogenen Bestandteile erhoben. Vor dem Hintergrund der Vielfalt klassischer und zum Teil auch neuer Studienrichtungen verstehen wir diese als Bestandteile der Studienprogramme, die entweder handlungsfeldbezogen sind (z.B. Sozialpädagogik, Erwachsenenbildung, Rehabilitationspädagogik) oder sich auf Handlungsformen beziehen (z.B. Didaktik, Organisation, Beratung).

Ein vierter Bereich von Studieninhalten sind die Praktika, die im Zuge der Bologna-Reform zur Sicherung der »Employability« von Studierenden auch in nicht zwangsläufig auf unmittelbare Verwertbarkeit ausgelegten universitären Studiengängen beitragen sollen (vgl. Schubarth/Speck et al. 2012; Schubarth/ Speck 2014). Dieser Aspekt wurde in Anbetracht der steigenden Studierendenzahlen auch vor der Bologna-Reform immer wieder diskutiert und schlug sich schon in der Rahmenordnung für die Diplomstudiengänge in Form einer Forderung nach breiteren Berufspraktika nieder (vgl. Rauschenbach 1994). Im Kerncurriculum werden für Bachelorstudiengänge Praktika im Umfang von $30 \mathrm{LP}$ und auf der Masterebene im Umfang von $14 \mathrm{LP}$ empfohlen. In diese Umfänge werden neben den reinen Praktika auch vor- und nachbereitende bzw. begleitende Lehrveranstaltungen sowie Praktikumsberichte als »integrale Bestandteile des Praktikums« (DGfE 2010: 39) einbezogen.

Von allen Studiengängen wurden die Anteile dieser vier inhaltlichen Studienbereiche in LP erhoben. Die uni- und bivariaten Auswertungen dieser Daten

Substrate disziplinärer Kommunikation über die Gestaltung von Studiengängen und als vom Fachverband autorisierte Orientierungsinstrumente zur Verfügung.

10 Als Basis dienten die Modulhandbücher und ggf. die Prüfungs- und Studienordnungen sowie Studienverlaufspläne. Um die Reliabilität der erhobenen Daten sicherzustellen, wurde die Durchsicht der Dokumente parallel von zwei Personen durchgeführt und die jeweiligen Zuordnungen miteinander verglichen.

11 Studieneinheit 2 = Gesellschaftliche, politische und rechtliche Bedingungen von Bildung, Ausbildung und Erziehung in schulischen und nicht-schulischen Einrichtungen unter Einschluss internationaler Aspekte « und Studieneinheit $3=$ »Einführung in erziehungswissenschaftliche Studienrichtungen« (vgl. DGfE 2010: 11). 
wurden durch eine hierarchische Clusteranalyse mittels Ward-Methode ergänzt, um die Studiengänge bezogen auf die Frage, wie sie die verpflichtenden Anteile in Grundlagen, Forschungsmethoden, Studienrichtungen und Praktika miteinander kombinieren, in möglichst homogene Gruppen aufzuteilen, die sich weitestgehend voneinander unterscheiden. Möglich ist es damit, unterschiedliche Muster der inhaltlichen Ausgestaltung von Studiengängen herauszuarbeiten (vgl. Kapitel 1.4).

\subsubsection{Grundlagenbezogene Studieninhalte}

Für die grundlagenbezogenen Anteile gilt zunächst, dass insgesamt gut ein Viertel $(26 \%)$ der Studiengänge den Empfehlungen des Kerncurriculums nicht folgt und keine dieser grundlagenbezogenen Inhalte vorsieht. Erwartbar häufiger ist dies in Master- als in Bachelorstudiengängen ( $40 \mathrm{zu} 9 \%$ ) zu beobachten, womit die Masterstudiengänge noch deutlicher als in der letzten Erhebung von diesen Inhalten absehen (Grunert et al. 2016: 29). Generalisierte Studiengänge bieten im BA alle und im MA zu 83 \% Grundlagenanteile an, während spezialisierte Studiengänge im BA zu $24 \%$ und im MA zu 53 \% keine verpflichtenden Grundlagenanteile enthalten. (vgl. Tabelle 1.1). Damit stellt ein Teil der spezialisierten Studiengänge weder einen namentlichen Bezug zur Erziehungswissenschaft noch in inhaltlicher Hinsicht zu disziplinären Grundlagen her. Im Spiegel dieser Fälle, insbesondere im Bachelorbereich, drängt sich erneut die Frage auf, inwieweit die Erziehungswissenschaft als Disziplin überhaupt noch als Referenzpunkt zur Ausgestaltung dieser Studienprogramme dient und inwiefern sie sich als erziehungswissenschaftliche Studiengänge verstehen.

Bei den Studiengängen, die Grundlagen im Studienprogramm vorsehen, schwanken die Umfänge beträchtlich. Größere Umfänge finden sich erwartbar vor allem in generalisierten BA-Studiengängen, von denen 43 \% 19 LP und mehr verpflichtend vorsehen, obwohl dies immerhin $10 \%$ weniger sind als zum Wintersemester 2014/15. Allerdings finden sich in diesem zeitlichen Vergleich nun auch mehr Studiengänge, die Grundlagen im Umfang von 13 bis 18 LP integrieren (vgl. Tabelle 1.1; Grunert et al. 2016: 31). Die spezialisierten BA-Studiengänge unterschreiten, wenn sie überhaupt Grundlagen vorsehen, die empfohlenen Umfänge des KCE zu $36 \%$, während dies nur für $11 \%$ der generalisiert gelabelten BA-Studiengänge zutrifft.

Im Gegensatz zum BA liegen die Leistungspunkte im MA häufiger im unteren Bereich von 1 bis 6 LP (generalisierte: 23 \%; spezialisierte: $25 \%$ ) bzw. 7 bis 12 LP (generalisierte: 38 \%; spezialisierte: $12 \%$ ), während Umfänge von 19 LP und mehr sich mit einer Ausnahme nur bei generalisierten Masterstudiengängen finden $(10 \%)$.

Bei der Betrachtung der Leistungspunktedifferenzen müssen natürlich auch die unterschiedlichen Gesamtumfänge der Studiengänge berücksichtigt 
werden (vgl. Kapitel 1.1). Aber auch dann, wenn man sich die prozentualen Anteile am Gesamtumfang der Studiengänge anschaut, bleiben die beschriebenen Tendenzen bestehen. Interessant erscheint dabei, dass insbesondere in generalisiert gelabelten Zwei-Fach-Studiengängen Grundlagen anteilig einen hohen Stellwert einnehmen, da hier ähnlich hohe Leistungspunkte vorgesehen sind wie in generalisierten Ein-Fach-Studiengängen.

Insgesamt haben sich damit im Vergleich zur letzten Erhebung im BA die Anteile an Grundlagen zwar nur leicht, aber zugunsten etwas höherer Anteile in den generalisierten BA-Studiengängen verschoben, während im Master die generalisierten Studiengänge nun in geringerem Maße Grundlagenanteile integrieren.

\subsubsection{Forschungsmethodenbezogene Studieninhalte}

Für die Ausbildungsanteile in qualitativen und quantitativen Forschungsmethoden zeigt sich, dass diese weiterhin in nahezu allen BA-Studiengängen (94\%) und im MA-Bereich zu $76 \%$ verpflichtend vorgesehen sind. Auch hier schwanken die Umfänge deutlich, wenngleich sie sich wie in der letzten Erhebung im BA bei 7 bis 12 LP konzentrieren und damit deutlich über den Empfehlungen des Kerncurriculums liegen (vgl. Tabelle 1.1). Im Master sind erneut vor allem in den generalisierten Studiengängen höhere Umfänge zu verzeichnen, was auf eine stärkere Forschungsorientierung in diesen Studiengängen verweist. Spezialisierte Studiengänge enthalten erwartbar vor allem dann einen hohen Anteil an Forschungsmethoden, wenn auch ihre Studienfachbezeichnung einen Forschungsbezug beinhaltet (z.B. »empirische Bildungsforschung").

Etwa die Hälfte der Studiengänge, die Forschungsmethoden integrieren, sehen eine gleichwertige Ausbildung sowohl in quantitativen als auch qualitativen Methoden vor. Eine ausschließlich qualitative Ausrichtung findet sich lediglich in drei Masterstudiengängen, eine ausschließlich quantitative Ausrichtung in zwei BA- und fünf MA-Studiengängen. Studiengänge, die die quantitativen Methoden stärker gewichten als die qualitativen, finden sich zu $32 \%$ im BA- und zu 12,5 \% im MA-Bereich, während nur drei Masterstudiengänge die qualitativen Methoden zu höheren Anteilen integrieren. $12 \%$ der Bachelor- und $21 \%$ der Masterstudiengänge überlassen schließlich den Studierenden die Schwerpunktsetzung innerhalb der forschungsmethodischen Ausrichtung. Lehrforschungsprojekte, verstanden als weitestgehend eigenständige Forschungsprojekte, die von Lehrenden begleitet und unterstützt werden, finden sich erwartungsgemäß eher in Masterstudiengängen und dabei zu höheren Anteilen vor allem in den spezialisierten (vgl. Tabelle 1.1). Im Vergleich zum Wintersemester 2014/15 zeigt sich jedoch auch, dass generalisierte Studiengänge sowohl im Bachelor- als auch im Masterbereich zunehmend Lehrforschungsprojekte verpflichtend im Studienprogramm verankern. 
Tabelle 1.1: Verpflichtende Studienanteile nach Inhaltsbereichen und Leistungspunkten ( $\mathrm{n}=194$; in \%, in Klammern: Wintersemester 2014/15)

\begin{tabular}{|c|c|c|c|c|c|c|c|c|}
\hline & \multicolumn{2}{|c|}{$\begin{array}{c}\text { BA generalisiert } \\
\qquad(n=53)\end{array}$} & \multicolumn{2}{|c|}{$\begin{array}{c}\text { BA spezialisiert } \\
(n=33)\end{array}$} & \multicolumn{2}{|c|}{$\begin{array}{l}\text { MA generalisiert } \\
\qquad(n=40)\end{array}$} & \multicolumn{2}{|c|}{$\begin{array}{c}\text { MA spezialisiert } \\
\quad(n=68)\end{array}$} \\
\hline \multicolumn{9}{|c|}{ Grundlagen } \\
\hline $0 \mathrm{LP}$ & - & $(-)$ & 24,2 & $(25,0)$ & 17,5 & $(8,1)$ & 52,9 & $(51,5)$ \\
\hline $1-6 \mathrm{LP}$ & 11,3 & $(7,5)$ & 36,4 & $(40,6)$ & 22,5 & $(21,6)$ & 25,0 & $(24,2)$ \\
\hline 7-12 LP & 15,1 & $(24,5)$ & 21,2 & $(18,8)$ & 37,5 & $(40,5)$ & 11,8 & $(10,6)$ \\
\hline 13-18 LP & 30,2 & $(15,1)$ & 3,0 & $(6,3)$ & 12,5 & $(13,5)$ & 8,8 & $(12,1)$ \\
\hline$\geq 19 \mathrm{LP}$ & 43,4 & $(52,8)$ & 15,2 & $(9,4)$ & 10,0 & $(16,2)$ & 1,5 & $(1,5)$ \\
\hline \multicolumn{9}{|c|}{ Forschungsmethoden } \\
\hline $0 \mathrm{LP}$ & - & $(-)$ & 15,2 & $(15,6)$ & 12,8 & $(13,9)$ & 29,4 & $(31,8)$ \\
\hline $1-6 \mathrm{LP}$ & 7,5 & $(7,5)$ & 21,2 & $(21,9)$ & 10,3 & $(5,6)$ & 17,6 & $(13,6)$ \\
\hline $7-12 \mathrm{LP}$ & 50,9 & $(47,2)$ & 42,4 & $(46,9)$ & 30,8 & $(30,6)$ & 29,4 & $(31,8)$ \\
\hline $13-18 \mathrm{LP}$ & 22,6 & $(20,8)$ & 18,2 & $(12,5)$ & 25,6 & $(22,2)$ & 8,8 & $(10,6)$ \\
\hline$\geq 19 \mathrm{LP}$ & 18,9 & $(24,5)$ & 3,0 & $(3,1)$ & 20,5 & $(27,8)$ & 14,7 & $(12,1)$ \\
\hline \multicolumn{9}{|c|}{ Lehrforschung } \\
\hline $0 \mathrm{LP}$ & 69,8 & $(78,8)$ & 78,8 & $(78,1)$ & 40,0 & $(62,2)$ & 41,2 & $(43,9)$ \\
\hline $1-6 \mathrm{LP}$ & 13,2 & $(13,5)$ & 9,1 & $(6,3)$ & 15,0 & $(5,4)$ & 4,4 & $(6,1)$ \\
\hline 7-12 LP & 11,3 & $(3,8)$ & 3,0 & $(9,4)$ & 22,5 & $(24,3)$ & 20,6 & $(25,8)$ \\
\hline 13-18 LP & 1,9 & $(1,9)$ & 3,0 & $(3,1)$ & 5,0 & $(2,7)$ & 25,0 & $(16,7)$ \\
\hline$\geq 19 \mathrm{LP}$ & 3,8 & $(1,9)$ & 6,1 & $(3,1)$ & 17,5 & $(5,4)$ & 8,8 & $(7,6)$ \\
\hline \multicolumn{9}{|c|}{ Studienrichtungen: Pflicht \& Wahlpflicht } \\
\hline$\leq 15 \mathrm{LP}$ & 13,1 & $(13,2)$ & - & $(-)$ & 20,0 & $(16,2)$ & 5,9 & $(4,6)$ \\
\hline $16-30 \mathrm{LP}$ & 37,7 & $(37,7)$ & - & $(-)$ & 35,0 & $(32,4)$ & 8,8 & $(13,6)$ \\
\hline $31-45 \mathrm{LP}$ & 22,6 & $(24,5)$ & 9,1 & $(6,3)$ & 32,5 & $(37,8)$ & 30,9 & $(27,3)$ \\
\hline $46-60 \mathrm{LP}$ & 20,8 & $(18,9)$ & 18,2 & $(18,8)$ & 7,5 & $(8,1)$ & 32,4 & $(31,8)$ \\
\hline$\geq 61 \mathrm{LP}$ & 5,7 & $(5,7)$ & 72,8 & $(75,0)$ & 5,0 & $(5,4)$ & 22,1 & $(22,7)$ \\
\hline \multicolumn{9}{|c|}{ Praktika } \\
\hline $0 \mathrm{LP}$ & 5,7 & $(5,7)$ & 6,1 & $(3,1)$ & 50,0 & $(45,9)$ & 47,1 & $(50,0)$ \\
\hline $1-6 \mathrm{LP}$ & 9,4 & $(11,3)$ & 6,1 & $(3,1)$ & - & $(-)$ & 1,5 & $(-)$ \\
\hline $7-12 \mathrm{LP}$ & 30,2 & $(28,3)$ & 12,1 & $(18,8)$ & 22,5 & $(27,0)$ & 27,9 & $(22,7)$ \\
\hline 13-18 LP & 32,1 & $(32,1)$ & 27,31 & $25,0)$ & 22,5 & $(21,6)$ & 16,2 & $(18,2)$ \\
\hline 19-29 LP & 18,9 & $(17,0)$ & 30,3 & $(28,1)$ & 5,0 & $(5,4)$ & 5,9 & $(4,5)$ \\
\hline$\geq 30 \mathrm{LP}$ & 3,8 & $(5,7)$ & 18,2 & $(21,9)$ & - & $(-)$ & 2,9 & $(3,0)$ \\
\hline
\end{tabular}


Betrachtet man auch hier noch einmal die prozentualen Anteile am Gesamtumfang der Studiengänge und addiert dafür die Anteile für Lehrforschungsprojekte und Forschungsmethoden, dann enthalten die Studiengänge verpflichtend durchschnittlich etwa $15 \%$ forschungsbezogene Anteile. In dieser Gesamtschau finden sich dann immerhin 13 Studiengänge $\left(\mathrm{n}_{\mathrm{BA}}=5 ; \mathrm{n}_{\mathrm{MA}}=8\right)$, die keinerlei verpflichtende forschungsbezogene Anteile beinhalten und es sind nach wie vor die Masterstudiengänge, die hier höhere Anteile als Bachelorstudiengänge aufweisen.

\subsubsection{Studienrichtungsbezogene Studieninhalte}

Nimmt man grundlagen- und forschungsbezogene Anteile zusammen, dann zeigt sich auch in der aktuellen Erhebung, dass vorwiegend spezialisierte Studiengänge gänzlich auf solche Studienbestandteile verzichten oder diese gegenüber generalisierten Studiengängen in deutlich geringerem Umfang integrieren und sich stärker auf die studienrichtungsbezogenen Studieninhalte konzentrieren. Insgesamt enthalten aber alle aktuellen Studiengänge solche studienrichtungsbezogenen Anteile. Knapp die Hälfte der Studiengänge (48,5 \%) sieht diese sowohl verpflichtend als auch wahlverpflichtend vor (d.h. mit der Möglichkeit einer individuellen inhaltlichen Schwerpunktsetzung), knapp $40 \%$ ausschließlich verpflichtend und schließlich $12 \%$ (vorwiegend im MA) nur wahlverpflichtend. Damit haben sich im Vergleich zum Wintersemester 2014/15 die Pflichtanteile in den Studiengängen deutlich erhöht und sind Wahlmöglichkeiten reduziert worden. Spezialisierte Studiengänge sowohl auf der Bachelor- als auch der Masterebene enthalten erwartbar mehr Leistungspunkte insbesondere in den verpflichtenden studienrichtungsbezogenen Anteilen und entsprechend weniger Wahlpflicht-Angebote (vgl. Tabelle 1.2).

Dieser Befund ist jedoch kaum überraschend, verweisen diese Studiengänge doch mehrheitlich bereits im Label auf ihre jeweiligen Schwerpunktsetzungen. Jedoch unterscheiden sich dabei wie in der letzten Erhebung (vgl. Grunert et al. 2016: 33) etwa rehabilitationspädagogische Studienprogramme, von denen fast $70 \%$ auch individuelle Schwerpunktsetzungen vorsehen, von sozialpädagogischen, die dies nur zu 35 \% tun. Möglicherweise ist dies ein Hinweis auf bereits in sich stark ausdifferenzierte Teildisziplinen und/oder breitere personelle Ressourcen, die eine breitere Palette an Schwerpunktsetzungen ermöglichen.

Auch in diesem Bereich schwanken die vorgesehenen Umfänge weiterhin erheblich und liegen zwischen 6 und 130 LP im BA und zwischen 9 und 87 LP im MA. Verglichen mit den Empfehlungen des Kerncurriculums, die im Bachelor36 und im Masterbereich 24 LP vorsehen, ${ }^{12}$ entsprechen die spezialisierten Stu-

12 Für Zwei-Fach-Studiengänge finden sich nur indirekt Empfehlungen im KCE, denn als Bezugsgröße dient hier Erziehungswissenschaft als Zweitfach, das ein größeres Erstfach voraussetzt. Hinzukommt, dass das Kerncurriculum dabei auch von einheitlichen Gesamtumfängen im Bereich der Zwei-Fach-Studiengänge ausgeht, was mit Blick auf die aktuellen Studiengän- 
diengänge alle dieser vorgeschlagenen Norm bzw. überschreiten diese, während generalisierte Bachelorstudiengänge den Empfehlungen für die studienrichtungsbezogenen Anteile nur zu einem Drittel entsprechen (vgl. Tabelle 1.1 und 1.2). Insgesamt sind neben den übergreifenden Differenzen zwischen generalisierten und spezialisierten Studiengängen auch deutliche Unterschiede zwischen Ein- und Zwei-Fach-Studiengängen zu beobachten, da Letztere nachvollziehbar deutlich geringere Anteile an studienrichtungsbezogenen Anteilen in die Studienprogramme integrieren. Allerdings ist hier auch zu berücksichtigen, dass Zwei-Fach-Studiengänge mehrheitlich generalisierte Studienfachbezeichnungen tragen und sich stärker auf Grundlagen und Forschungsmethoden konzentrieren.

Tabelle 1.2: Verpflichtende Studienanteile nach Leistungspunkten: Studienrichtung Pflicht und Wahlpflicht ( $\mathrm{n}=194$; in \%)

\begin{tabular}{|c|c|c|c|c|c|c|c|c|}
\hline & \multicolumn{2}{|c|}{$\begin{array}{l}\text { BA generalisiert } \\
\qquad(n=53)\end{array}$} & \multicolumn{2}{|c|}{$\begin{array}{l}\text { BA spezialisiert } \\
\qquad(n=33)\end{array}$} & \multicolumn{2}{|c|}{$\begin{array}{l}\text { MA generalisiert } \\
\qquad(n=40)\end{array}$} & \multicolumn{2}{|c|}{$\begin{array}{l}\text { MA spezialisiert } \\
\qquad(n=68)\end{array}$} \\
\hline & Pflicht & $\begin{array}{l}\text { Wahl- } \\
\text { pflicht }\end{array}$ & Pflicht & $\begin{array}{l}\text { Wahl- } \\
\text { pflicht }\end{array}$ & Pflicht & $\begin{array}{l}\text { Wahl- } \\
\text { pflicht }\end{array}$ & Pflicht & $\begin{array}{l}\text { Wahl- } \\
\text { pflicht }\end{array}$ \\
\hline $0 \mathrm{LP}$ & 3,8 & 39,6 & - & 45,5 & 50,0 & 22,5 & 4,4 & 45,6 \\
\hline 1-9 LP & 22,6 & 3,8 & - & 6,1 & 12,5 & 5,0 & 5,9 & 4,4 \\
\hline 10-15 LP & 26,4 & 26,4 & - & 12,1 & 20,0 & 10,0 & 11,8 & 11,8 \\
\hline $16-30 \mathrm{LP}$ & 17,0 & 22,6 & 6,1 & 24,2 & 12,5 & 32,5 & 22,1 & 26,5 \\
\hline $31-45$ LP & 15,1 & 5,7 & 24,2 & 6,1 & 5,0 & 22,5 & 32,4 & 10,3 \\
\hline $46-60$ LP & 13,2 & 1,9 & 21,2 & - & - & 7,5 & 14,7 & 1,5 \\
\hline $61-90$ LP & 1,9 & - & 24,2 & 6,1 & - & - & 8,8 & 6,1 \\
\hline$\geq 91 \mathrm{LP}$ & - & - & 24,2 & - & - & - & - & - \\
\hline
\end{tabular}

Grundsätzlich setzen sich in inhaltlicher Perspektive bei den wählbaren Studienrichtungen in generalisierten Bachelor- und Masterstudiengängen die bereits in den letzten Datenreporten (vgl. Stisser et al. 2012; Grunert et al. 2016) beschriebenen Trends weiterhin fort: Studienrichtungen mit eigenen Empfehlungen im Kerncurriculum (Erwachsenenbildung/Weiterbildung, Sozialpädagogik, Sonderpädagogik, Pädagogik der frühen Kindheit) finden sich auch am ehesten in den Studiengängen wieder (vgl. Abbildung 1.4). Bei der nach wie vor mit Abstand am häufigsten wählbaren Studienrichtung Erwachsenenbildung/ Weiterbildung, die zugleich am seltensten auch in eigenen Studiengängen $\mathrm{zu}$ beobachten ist, zeigt sich in der aktuellen Datenerhebung jedoch nicht nur ein leichter Rückgang bezogen auf eigenständige Studiengänge (vgl. Kapitel 1.2), sondern auch mit Blick auf die Reduktion von Studiengängen, innerhalb de- 
rer die Studienrichtung angeboten wird. Für die Kindheitspädagogik und die Sozialpädagogik zeigt sich hingegen auf der Ebene von eigenständigen Studiengängen insbesondere im Masterbereich ein leichter Zuwachs, und auch auf der Ebene der wählbaren Studienrichtungen in generalisierten Studiengängen zeigt sich aktuell eher eine Konstanz. Studienrichtungen im Feld der Interkulturellen Bildung, empirischen Bildungsforschung und der Pädagogik der Lebensalter sind insgesamt nun etwas häufiger in den Studiengängen enthalten, wobei sich dies vor allem auf die Integration in das Wahlangebot von Masterstudiengängen zurückführen lässt.

Abbildung 1.4: Wählbare Studienrichtungen in generalisierten Studiengängen ( $\mathrm{n}=64$; in absoluten Häufigkeiten)

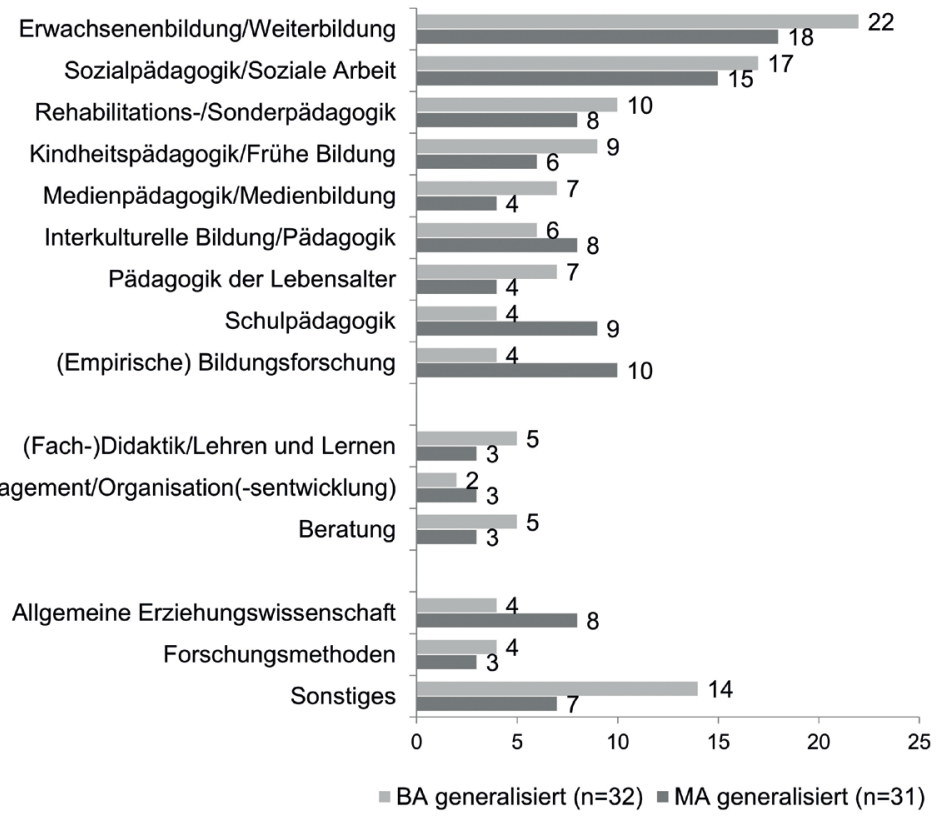

Bezogen auf die Wahlkombinationen an den einzelnen Standorten führt die Möglichkeit, sich in einem Studiengang mindestens für Erwachsenenbildung oder Sozialpädagogik entscheiden zu können, die Auswahlliste an (22 Studiengänge). Gefolgt wird dies von der Option, mindestens zwischen Erwachsenenbildung oder Rehabilitationspädagogik (12 Studiengänge) sowie zwischen Erwachsenenbildung oder Kindheitspädagogik (8 Studiengänge) wählen zu können. In elf Studiengängen werden die klassischen Studienrichtungen So- 
zialpädagogik, Rehabilitationspädagogik und Erwachsenenbildung/Weiterbildung gleichzeitig zur Wahl gestellt. Alle weiteren denkbaren Varianten von Auswahlmöglichkeiten treten demgegenüber eher vereinzelt auf und folgen keinem erkennbaren Muster. Auch in der Frage, wie viele Studienrichtungen überhaupt zur Auswahl stehen, ist keine Tendenz auszumachen. Dies kann je nach Studiengang und Standort zwischen 2 und 16 variieren, wobei mehr als 5 zur Auswahl stehende Studienrichtungen deutlich seltener werden und nur vereinzelt vorkommen.

Insgesamt verweisen die vielfältigen Schwerpunktsetzungen und Kombinationsmöglichkeiten auch weiterhin auf sehr unterschiedliche Profilbildungen an den Standorten und zeugen einmal mehr von der Ausdifferenzierung der Erziehungswissenschaft, die sich in einem breiten Spektrum differenter Handlungsfelder zeigt.

\subsubsection{Praktikumsbezogene Studieninhalte}

Vergleichsweise homogen erscheinen die Studiengänge jedoch in der Frage der Praktika, die konstant zur letzten Erhebung in insgesamt $94 \%$ der BAund etwa $50 \%$ der MA-Studiengänge verpflichtend vorgesehen sind, jedoch in den spezialisierten Masterstudiengängen etwas stärker an Bedeutung gewonnen haben (vgl. Grunert et al. 2016: 35). Die Umfänge der Praktika schwanken zwischen 3 und 24 Wochen sowohl im Bachelor als auch im Master. Nicht immer sind in diese Praktika, wie im KCE empfohlen, begleitende Lehrveranstaltungen integriert, wobei dies wiederum sehr viel häufiger in Master- als in Bachelorstudiengängen zu beobachten ist (36 bzw. $21 \%$ ). Differenzen in den vorgesehenen Praktika zwischen generalisierten und spezialisierten Studiengängen zeigen sich weiterhin vorrangig auf der Bachelorebene, da v.a. spezialisierte Studienprogramme häufiger größere Umfänge integrieren und damit auch ihren Handlungsfeldbezug schärfer konturieren (vgl. Tabelle 1.1). Kindheitspädagogische Studienprogramme sehen dabei mehrheitlich Praktika von über zwölf Wochen vor $(72 \%)$. Ähnlich lange Praktika finden sich, wenn auch deutlich seltener (57 \%), nur noch in sozialpädagogischen Studiengängen. Alle generalisierten Studiengänge zusammengenommen sehen dies dagegen nur zu 13 \% vor. Einmal mehr verweist dies darauf, dass der Ausbau von Studiengängen im Bereich der frühen Kindheit in engem Zusammenhang mit handlungsfeldbezogenen Anforderungen steht (vgl. Stisser et al. 2012; Züchner 2012). Blickt man noch einmal auf den Masterbereich, sind die Praktika hingegen anders als im Bachelor häufiger als Forschungspraktikum ausgelegt (17 \%; BA: nur ein Studiengang).

Insgesamt verweisen die bisherigen Befunde auf eine sehr unterschiedliche Verteilung inhaltlicher Studienbestandteile und Schwerpunktsetzungen und nur geringfügige Veränderungen der Studienprogramme, die sich vor allem im 
Grundlagen- und Praktikumsbereich bemerkbar machen. Wie diese verschiedenen Inhalte zueinander im Verhältnis stehen und welche Gewichtungen in den Studienprogrammen vorgenommen werden, dem soll im Folgenden nachgegangen werden. Vor dem Hintergrund der dargestellten unterschiedlichen Gesamtumfänge sowie der Unterschiede zwischen Ein-Fach- und Zwei-FachStudiengängen, halten wir am Vorgehen aus der letzten Erhebung fest und legen dafür die prozentualen Anteile am Gesamtumfang der Studiengänge zugrunde (vgl. Grunert et al. 2016: 35).

\subsection{Studiengangsmuster}

\subsubsection{Bachelorstudiengänge}

Sowohl im Bachelor- als auch im Masterbereich konnten auf der Basis einer Clusteranalyse erneut jeweils fünf verschiedene Muster von Studiengängen daraufhin unterschieden werden, wie sie die Anteile an erziehungswissenschaftlichen Grundlagen, Forschungsmethoden und Studienrichtungen sowie Praktika in ihren Studienprogrammen miteinander kombinieren. ${ }^{13}$ Im BA-Bereich gleichen die herausgearbeiteten Muster denen aus der letzten Erhebung, während im Master einige Verschiebungen zu verzeichnen sind (vgl. Grunert et al. 2016: 35ff.).

Im Bachelorbereich findet sich dabei zunächst eine Gruppe von Studiengängen mit durchschnittlich relativ ähnlichen Anteilen im Bereich der Grundlagen, Forschungsmethoden sowie Praktika (13, 10 bzw. 12 \%). Wenngleich studienrichtungsbezogene Inhalte mit $25 \%$ eher in unterdurchschnittlichem Maße integriert werden, kann dieses Muster als paritätisch-ausgewogen bezeichnet werden (vgl. Abbildung 1.5). Diese Studiengänge entsprechen damit annähernd auch den Strukturempfehlungen des Kerncurriculums, obwohl hier grundlagen- und forschungsmethodische Bezüge stärker als vorgeschlagen berücksichtigt werden. Dieses Muster findet sich insgesamt in etwa einem Drittel der Studienprogramme und speist sich fast vollständig aus generalisierten Studiengängen, sowohl in der Ein-Fach- als auch in der Zwei-Fach-Struktur (vgl. Abbildung 1.6).

Das zweite Muster stark grundlagenbezogener Studienprogramme findet sich ausschließlich in generalisierten Studiengängen und bis auf eine Ausnahme auch nur innerhalb der Zwei-Fach-Studiengänge. Diese Gruppe zeichnet sich vor allem dadurch aus, dass hier besonders stark auf die Grundlagen abgestellt wird, die durchschnittlich $26 \%$ des Studienprogramms ausmachen. Während hier die Praxis- und Studienrichtungsanteile den geringsten Stellenwert

13 Grundlage der Clusteranalyse sind die z-standardisierten prozentualen Anteile der verpflichtenden Bestandteile am Gesamtumfang des Studienprogramms (exkl. Nebenfächer und Studium Generale) und damit Abweichungen vom Mittelwert, die jeweils getrennt für die BAund MA-Studiengänge ausgewertet wurden. 
einnehmen, sind auch die forschungsmethodischen Bezüge überdurchschnittlich hoch vertreten.

Abbildung 1.5: Bachelor-Studiengangsmuster ( $\mathrm{n}=86$; links $\mathrm{z}$-standardisierte Abweichungen vom Mittelwert der prozentualen Pflichtanteile am Gesamtumfang, rechts durchschnittliche prozentuale Anteile am Gesamtumfang)

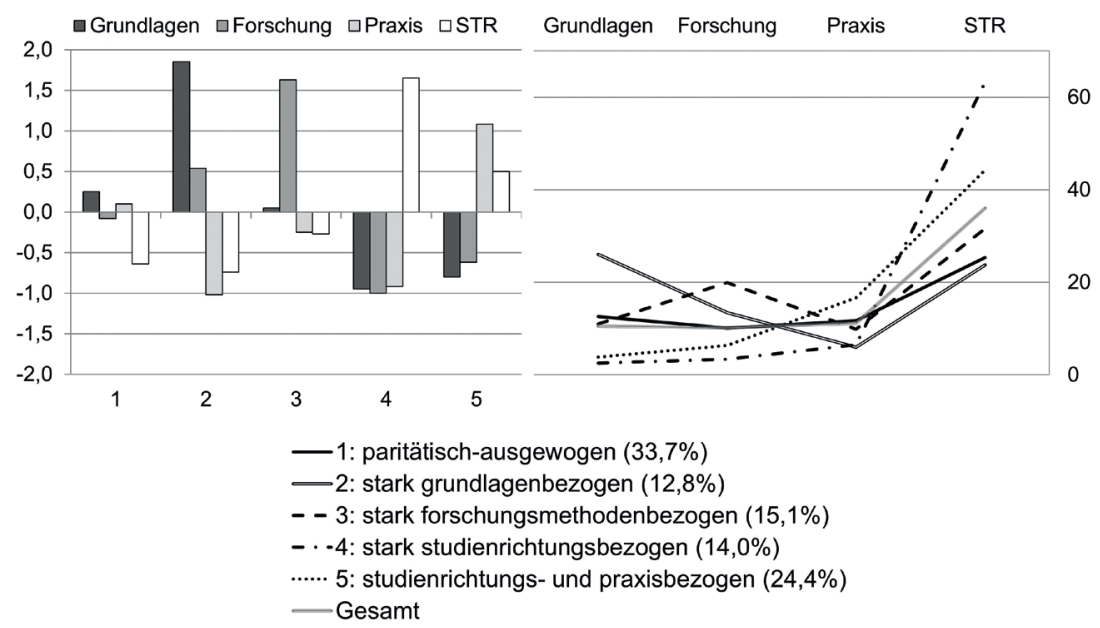

Ein Pendant dazu findet sich im dritten Muster von Bachelorstudiengängen. Bei diesen stark forschungsmethodenbezogenen Studienprogrammen dreht sich die Gewichtung von Grundlagen und Forschungsmethoden um. Praktika und Studienrichtungen werden zwar immer noch unterdurchschnittlich, aber gegenüber den stark grundlagenbezogenen Studiengängen in höherem Maße integriert. Letztlich finden sich auch in dieser Gruppe mit mehr als drei Viertel (77 \%) deutlich mehr generalisierte Studiengänge, wobei hier etwa ähnlich viele Ein-Fach- wie Zwei-Fach-Studiengänge vertreten sind.

Schließlich finden sich zwei Muster mit vorwiegend spezialisierten und jeweils mit einem Verhältnis von etwa zwei Dritteln zu einem Drittel etwas mehr Ein-Fach- als Zwei-Fach-Studiengängen. In beiden Gruppen sind Grundlagen und Forschungsmethoden allenfalls marginale Bestandteile des Studienprogramms (vgl. Abbildung 1.5). Demgegenüber legen diese Studiengänge ihren Fokus auf die studienrichtungsbezogenen Anteile, wobei die stark studienrichtungsbezogenen Studienprogramme, wie auch die meisten grundlagenbezogenen Studiengänge, Praktika mit durchschnittlich 6,5 \% in eher moderatem Umfang vorsehen. Bei den studienrichtungs- und praxisbezogenen Studien- 
gängen bilden Praktika hingegen einen Schwerpunkt innerhalb der Studienprogramme und verweisen damit auf einen deutlich anwendungsorientierten Handlungsfeldbezug.

Abbildung. 1.6: Häufigkeit von Bachelor-Studiengangsmustern nach generalisierten und spezialisierten Studiengängen (links generalisierte $(n=53)$, rechts spezialisierte $(n=33)$, in \%)

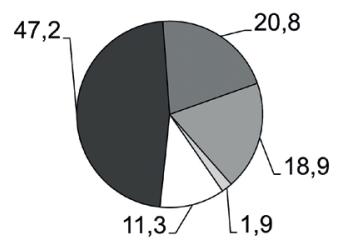

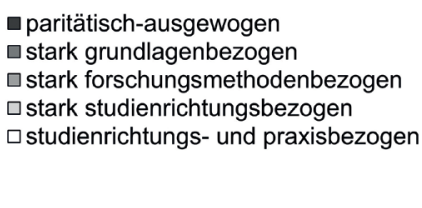

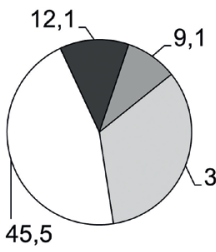

33,3

Wenngleich sich die Studiengangsmuster im Bachelorbereich überwiegend entlang der Linie generalisierter und spezialisierter Studienfachbezeichnungen verteilen, lassen sich dennoch sehr unterschiedliche Muster nachweisen, die darauf aufmerksam machen, dass die Empfehlungen des Kerncurriculums kaum als verbindliche Bezugsgröße für die Ausgestaltung der Studiengänge herangezogen wurden (vgl. auch Ludwig/Grunert 2020). Bachelorstudiengänge in der Erziehungswissenschaft bzw. ihren Teildisziplinen sind damit weiterhin strukturell wie auch inhaltlich in hohem Maße heterogen ausgestaltet und auch die jeweilige Studienfachbezeichnung bietet allenfalls eine grobe Orientierungshilfe und lässt nicht zwangsläufig auf integrierte Inhalte und deren Gewichtungen schließen. Offen ist nun, inwieweit sich dieser Eindruck auch in den Masterstudiengängen wiederfindet.

\subsubsection{Masterstudiengänge}

Im Masterbereich ließen sich ebenfalls erneut fünf Muster an Studiengangsprofilen unterscheiden, die auf verschiedene inhaltliche Schwerpunktsetzungen in Bezug auf die untersuchten Kriterien verweisen. Dabei fällt zunächst auf, dass im Vergleich zur letzten Erhebung nicht mehr in vier, sondern in drei von fünf Mustern die inhaltlichen Anteile ähnlich relationiert sind, sodass höhere Forschungs- und Studienrichtungsanteile mit geringeren Grundlagenund Praxisanteilen einhergehen. Unterschieden werden können diese Studiengangsmuster jedoch durch die differenten prozentualen Anteile dieser Inhalte an den jeweiligen Studienprogrammen. Ein weiteres Muster integriert nun neben etwas überdurchschnittlichen Forschungsmethoden auch deutlich höhere Praxisanteile, die insbesondere in den spezialisierten Masterstudiengängen 
bei der letzten Erhebung noch eine geringere Rolle spielten (vgl. Grunert et al. 2016: 36; hier: Abbildung 1.7).

Ein erstes Muster an Studiengängen legt auch im Masterbereich den Schwerpunkt auf allgemein-erziehungswissenschaftliche Inhalte, der mit einem durchschnittlichen Forschungs- und Praxisbezug einhergeht. Studienrichtungsbezogene Anteile sind hier klar unterdurchschnittlich vertreten. In diesem stark grundlagenbezogenen Muster finden sich deutlich mehr generalisierte als spezialisierte Studiengänge, wobei Letztere bis auf eine Ausnahme in der Bezeichnung entweder auf "Erziehungswissenschaft " oder allgemeiner »Bildung « verweisen. Im Vergleich zum affinen Muster der letzten Erhebung verstärken sich hier die Grundlagen- und Forschungsbezüge und verringern sich die Studienrichtungs- und Praxisanteile, sodass sich dieses Muster noch deutlicher als grundlagenbezogenes Studiengangsmuster konturiert.

Abbildung 1.7: Master-Studiengangmuster ( $\mathrm{n}=108$; links z-standardisierte Abweichungen vom Mittelwert der prozentualen Pflichtanteile am Gesamtumfang, rechts durchschnittliche prozentuale Anteile am Gesamtumfang)

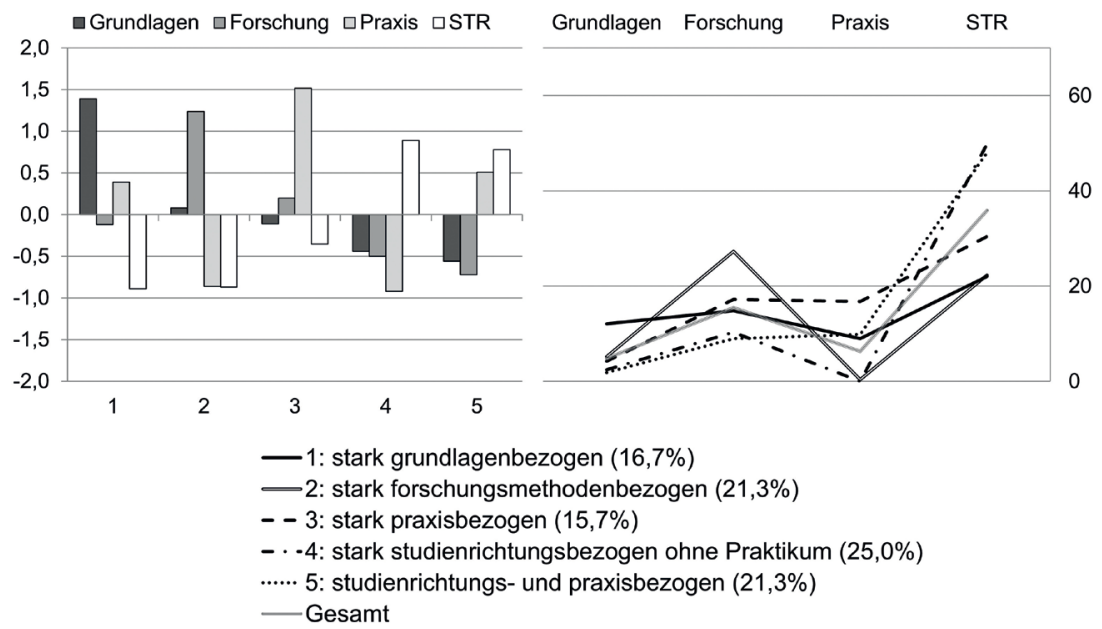

Das zweite Muster, die stark forschungsmethodenbezogenen Studienprogramme, zeichnet sich durch sehr hohe forschungsmethodische Anteile aus, die nur von durchschnittlichen Grundlagen- und deutlich unterdurchschnittlichen Studienrichtungsanteilen begleitet werden. Auf Praktika wird fast gänzlich verzichtet. Spezialisierte Studiengänge dieses Clusters verweisen im Label auf einen Forschungsschwerpunkt, mehrheitlich auch in Kombination zum Begriff 
"Erziehungswissenschaft«. Gleichzeitig finden sich aber auch Studiengänge, die der Bezeichnung nach auf Handlungsfelder wie Sozialpädagogik, Schule oder auch Frühe Kindheit verweisen. Im Vergleich zum affinen Muster der letzten Erhebung (vgl. Grunert et al. 2016: 38) schärft sich auch dieses Muster deutlicher aus, indem den Forschungsmethoden ein noch stärkeres und den anderen Bereichen ein geringeres Gewicht zukommt.

Eine dritte Gruppe zeichnet sich darüber aus, dass sie etwas überdurchschnittliche Anteile in Forschungsmethoden mit deutlich überdurchschnittlichen Praxisanteilen kombiniert. Forschungsmethoden sind dabei im Vergleich zur letzten Erhebung etwas geringer und Praktika deutlich höher gewichtet, sodass sich dieses Muster eher in Richtung Praxisbezug konturiert. In dieser Gruppe konzentrieren sich auch Studiengänge, die ihre Praktika als Forschungspraktikum ausrichten, wobei dies vorrangig spezialisierte Studiengänge betrifft.

Und schließlich finden sich auch hier, wie im Bachelorbereich, zwei Gruppen, die vorrangig auf Studienrichtungen fokussieren. Auf der Masterebene geht dies jedoch weitestgehend mit dem Verzicht auf allgemein-erziehungswissenschaftliche Inhalte einher. Beide Muster unterscheiden sich letztlich darüber, dass in einem Cluster Praktika gänzlich fehlen, während sie im anderen integraler Bestandteil der Studienprogramme sind. Anders als im Bachelorbereich sind Studiengänge mit der Bezeichnung "Erziehungswissenschaft" hier etwas häufiger zu finden. Dies sind solche Studiengänge, die allgemeinerziehungswissenschaftliche Inhalte in ihrem Wahlpflichtbereich integrieren, der gleichzeitig auch Vertiefungen in Sozialpädagogik, Erwachsenenbildung, Rehabilitationspädagogik und weiteren Studienrichtungen ermöglicht. Im Vergleich zu den affinen Mustern aus der letzten Erhebung hat sich in beiden Clustern der Studienrichtungsbezug noch einmal deutlich erhöht.

Abbildung 1.8: Häufigkeit von Master-Studiengangsmustern nach generalisierten und spezialisierten Studiengängen (links generalisierte $(n=40)$, rechts spezialisierte $(n=68)$, in \%)

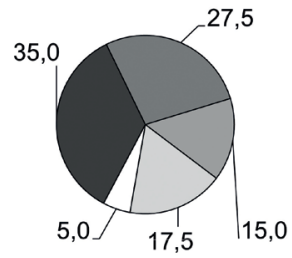

ustark grundlagenbezogen 匹 stark forschungsmethodenbezogen $\square$ stark praxisbezogen $\square$ stark studienrichtungsbezogen ohne Praktikum $\square$ studienrichtungs- und praxisbezogen

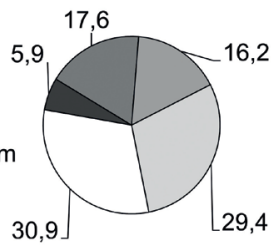

Insgesamt betrachtet, werden auf der Masterebene die Strukturempfehlungen des Kerncurriculums, abgesehen von Praktika, auch weiterhin eher überboten. Deutlicher als auf der Bachelorebene lässt sich jedoch nicht anhand der Studienfachbezeichnungen auf die Ausgestaltung der Studienprogramme schließen. 
Spezialisierte Studiengänge etwa, die dem Label nach der Sozialpädagogik, der Erwachsenenbildung, der Kindheitspädagogik oder dem Bildungsmanagement/Organisationsentwicklung zugeordnet werden können und auch generalisierte Studiengänge mit den Bezeichnungen Erziehungswissenschaft(en) oder Bildungswissenschaft(en), finden sich erneut in allen Mustern wieder, sodass sich auch die Trennlinie zwischen generalisierten und spezialisierten Studiengängen, die im Bachelor noch zu finden ist, hier wieder aufweicht (vgl. Abbildung 1.8). Im Vergleich zur Erhebung im Wintersemester 2014/15 haben sich über die Verschiebungen in den Studienprogrammen die Clusterstrukturen im Masterbereich jedoch noch einmal stärker konturiert. Während sich im Bachelorbereich im Zeitvergleich abzeichnet, dass sich von der Studienfachbezeichnung nicht zwangsläufig auf die Inhalte der Studiengänge schließen lässt und sich dies zum Wintersemester 2018/19 noch einmal deutlicher zeigt (vgl. Abbildung 1.6), lässt sich im Masterbereich zumindest für Studiengänge mit generalisierten Labels ein umgekehrter Trend erkennen. Hier finden sich nun knapp zwei Drittel der Studiengänge im stark grundlagen- bzw. stark forschungsbezogenen Cluster wieder (2014/15: 43,2 \%; Grunert et al. 2016: 39) und verweisen damit auf eine stärkere Passung zwischen Studienfachbezeichnungen und Studieninhalten.

\subsubsection{Inhaltliche Muster an Standorten?}

Betrachtet man die herausgearbeiteten Cluster im Bachelor- und Masterbereich bezogen auf ihre Kombination an den einzelnen Standorten, dann ist man erneut auf ein heterogenes Feld an unterschiedlichen Kombinationsmöglichkeiten verwiesen und entsprechend zeigen sich kaum übergreifende Strukturen. Bezieht man jedoch ausschließlich die Anteile an grundlagenbezogenen Inhalten ein, die insgesamt an den jeweiligen Standorten angeboten werden, dann lassen sich auch mit Blick auf die Studiengangslandschaft im Wintersemester 2018/19 vier zentrale Tendenzen erkennen.

Am häufigsten findet sich nach wie vor eine erste Gruppe von Standorten $(n=23)$, die sowohl im Bachelor als auch im Master auf eine grundlagenbezogene Ausbildung setzen - auch wenn im Vergleich zum Wintersemester 2014/15 hier eine Reduktion von zwei Standorten zu beobachten ist. Dies ist darauf zurückzuführen, dass ein Standort den Bachelorstudiengang eingestellt hat, zwei Standorte haben die verpflichtenden Grundlagenanteile im Masterstudiengang reduziert und ein weiterer Standort wiederum hat die Grundlagenanteile im Masterbereich erhöht. Im Zeitvergleich wird darüber deutlich, dass hier - wie auch in den anderen Gruppen - Bewegungen in unterschiedliche Richtungen zu beobachten sind. Ähnlich wie im Wintersemester 2014/15 finden sich in dieser ersten Gruppe mehrheitlich Standorte, die mindestens einen generalisierten Bachelor- und einen generalisierten Masterstudiengang anbieten $(n=17)$. 
Eine zweite Gruppe von Standorten ( $\mathrm{n}=16)$ verankert zwar im Bachelorstudium erziehungswissenschaftliche Grundlagen, misst diesen dagegen im Masterbereich weniger Bedeutung zu oder verzichtet gänzlich auf eine Fortführung dieser Inhalte. Angesichts der höheren Anteile im Bachelorbereich scheint ein stärkerer Verzicht auf der Masterebene jedoch durchaus nachvollziehbar und erinnert an das Modell des Grund- und Hauptstudiums in den Diplomstudiengängen, bei dem im Anschluss an eine Grundlagenausbildung eine zunehmende Spezialisierung vorgesehen war. Diesem Bild entspricht auch, dass hier mehrheitlich Standorte zu finden sind, die den jeweiligen Studienfachbezeichnungen nach mindestens einen generalisierten Bachelor- und einen spezialisierten Masterstudiengang anbieten $(n=9)$. Im Vergleich zum Wintersemester 2014/15 haben hier zwei Standorte ihre Grundlagenanteile im Bachelor erhöht und im Masterstudiengang reduziert, sodass diese nun in der zweiten Gruppe vertreten sind. An einem weiteren Standort wurden hingegen die Grundlagenanteile im Bachelorbereich reduziert, sodass dieser nunmehr in der vierten Gruppe enthalten ist. Auch hier setzt sich also das Bild gegenläufiger Bewegungen fort. Zusammengenommen machen diese ersten beiden Kombinationslinien gut $60 \%$ der Standorte aus.

Eine dritte Gruppe von Standorten integrieren erst im Masterbereich stärker erziehungswissenschaftliche Grundlagen. Dieses Muster findet sich allerdings an lediglich vier Standorten und ist möglicherweise der Verweis darauf, dass diese Standorte sich im Bachelorbereich stärker auf handlungsfeldbezogene Anforderungen orientieren und erst im Master ein disziplinäres Profil schärfen. Der Studienfachbezeichnung nach würde das allerdings nur auf einen Standort zutreffen, der im Bachelor einen spezialisierten Studiengang und auf der Masterebene sowohl einen spezialisierten als auch einen generalisierten Studiengang anbietet. Bewegungen in dieser Gruppe kommen im Zeitvergleich vor allem über eine Erhöhung von verpflichtenden Grundlagenanteilen im Bachelor- oder Masterbereich zustande, die zu einer veränderten Zuordnung von drei Standorten führt (zwei Standorte von Gruppe 3 in 2; ein Standort von Gruppe 4 in 3).

Schließlich finden sich elf Standorte und damit im Vergleich zum Wintersemester 2014/15 zwei Standorte mehr, die sowohl auf Bachelor- als auch auf Masterebene allenfalls marginale Grundlagenanteile vorsehen und entsprechend die Frage aufwerfen, ob Erziehungswissenschaft als Wissenschaftsdisziplin in dieser vierten Gruppe überhaupt ein Referenzpunkt für die Ausgestaltung von Studienprogrammen darstellt. Im Vergleich zum Wintersemester 2014/15 ist dies jedoch nicht auf studiengangsimmanente Veränderungen zurückzuführen, sondern hier finden sich nun zwei Standorte wieder, die aufgrund von Veränderungen im Studiengangsangebot erstmals in die Berechnungen aufgenommen wurden. Neun der elf Standorte bieten im Bachelor- und Masterbereich spezialisierte Studiengänge an. Vor diesem Hintergrund zeichnet sich in zeitlicher Perspektive eine Zunahme an Standorten ab, in denen die 
Vermittlung von Grundbegriffen, Theorien und Geschichte der Erziehungswissenschaft kaum noch eine Rolle spielt. Betrachtet man akademische Studiengänge jedoch als Medien der disziplinären Selbstreproduktion, die über die reine Wissensvermittlung hinausgeht, indem immer auch disziplinbezogene Normen und Werte vermittelt werden, dann geschieht dies an einigen Standorten nicht mehr im Hinblick auf die Gesamtdisziplin, sondern primär über teil- oder auch interdisziplinäre Wissenschaftskulturen.

\subsection{Fazit}

In der Zusammenschau macht auch der aktuelle Stand des Angebotes an erziehungswissenschaftlichen Bachelor- und Masterstudiengängen in Deutschland deutlich, dass der Dauerbefund der disziplinären Selbstbeobachtung der letzten Jahre nach wie vor seine Gültigkeit besitzt: Erziehungswissenschaftliche Studiengänge können auch weiterhin als vielfältig, ausdifferenziert, heterogen oder auch diversifiziert beschrieben werden (vgl. etwa Rauschenbach 1994; Grunert/Rasch 2006; Horn/Wigger/Züchner 2008; Grunert 2012; Stisser et al. 2012; Vogel 1994; Grunert et al. 2016) und bewegen sich in einem breiten Spektrum an strukturellen und inhaltlichen Ausgestaltungsvarianten.

Im Bereich der Bachelor- und Masterstudiengänge zeichnen sich dabei folgende zentrale Trends ab:

(1) Fast alle Studienstandorte bieten sowohl Bachelor- als auch Masterstudiengänge an, wenngleich das Masterangebot deutlich größer und bezogen auf die Fachbezeichnungen der Studiengänge inhaltlich ausdifferenzierter ist. Im Zeitvergleich setzt sich der Ausbau der Studiengänge weiterhin vor allem im Masterbereich fort. Auch wenn sich rein äußerlich die Zahl der Studiengänge insgesamt im Vergleich zum Wintersemester 2014/15 nur leicht erhöht hat, verweisen die Bewegungen innerhalb bestehender Studienprogramme auf eine auch gegenwärtig anhaltende, hohe Dynamik im Feld der erziehungswissenschaftlichen Studiengangslandschaft. Dies ist sicherlich auch auf die fortlaufenden Reakkreditierungsprozesse im Bachelor- und Mastersystem zurückzuführen. Dass in den damit einhergehenden Veränderungen der Studiengänge kein gemeinsamer Trend zu erkennen ist, sondern vielmehr auch gegenläufige Prozesse zum Ausdruck kommen, wirft die Frage auf, inwieweit die Fachgesellschaft bei aller begrüßenswerter Pluralität in stärkerem Maße auch für Akkreditierungsverfahren fachliche Leitorientierungen zur Diskussion stellen könnte (vgl. Grunert/Ludwig 2019).

(2) Die Fachbezeichnungen der Studiengänge weisen nach wie vor ein breites Spektrum auf. Heterogenität drückt sich dabei zum einen über eine differente Begriffsverwendung bereits auf der Ebene der generalisierten disziplinbezogenen Fachbezeichnungen aus und potenziert sich durch die Stu- 
diengänge, die sich in ihrem Label eher an Teildisziplinen bzw. Spezialthemen orientieren. Auch im Vergleich mit anderen Fächern, insbesondere der Soziologie (vgl. Grunert/Ludwig 2016a) wird darüber deutlich, dass sich die Erziehungswissenschaft in besonderem Maße über ihre symbolischen Grenzziehungen in Form von Studienfachbezeichnungen auch nach außen hin als fragmentierte Disziplin (Ambrose et al. 2010) zeigt. Teildisziplinäre Ausdifferenzierung und die Besetzung neuer Themen- und Praxisfelder verorten sich in den Studienfachbezeichnungen nicht nur im innerdisziplinären Diskurs, sondern werden als symbolische Grenzziehungen auch nach außen getragen. Über qualitative Untersuchungen (vgl. etwa Grunert/Ludwig 2016b; Ludwig/ Grunert 2018; Ludwig 2019) deutet sich dabei an, dass damit sowohl disziplinäre Positionierungs- und Abgrenzungskämpfe ausgetragen werden, als auch, dass das mit dem Bologna-Prozess transportierte Postulat des notwendigen Alleinstellungsmerkmals oder der Marktgängigkeit z.T. ohne Rückbindung an den disziplinären Diskurs bedient wird. Dass es sich dabei insbesondere bei den spezialisierten Studiengängen eher weniger um kollektive strategische teildisziplinäre Verselbstständigungsschritte zu handeln scheint, darauf verweisen die wiederum uneinheitlichen Begriffswahlen, etwa wenn es um Studiengänge im Kontext der Sozialpädagogik oder der Kindheitspädagogik geht.

(3) Bereits im Wintersemester 2014/15 konnte herausgestellt werden, dass ein generalisiertes Labeling nicht zwangsläufig mit stärkeren Bezügen auf disziplinbezogene Ausbildungsinhalte im Sinne erziehungswissenschaftlicher Grundlagen und Forschungsmethoden einhergeht. Mit den aktuellen Veränderungen in den Studienprogrammen, insbesondere im Grundlagen- und Praktikumsbereich, setzt sich dieser Trend weiter fort und führt vor allem im Bachelorbereich zu einer weiteren Unschärfe im Zusammenhang von Label und Inhalt der Studiengänge. Allerdings zeigen sich im Masterbereich auch gegenläufige Bewegungen, die auf eine leicht zunehmende Konturierung von generalisierten Studiengängen in diesem Punkt hindeuten.

(4) Auch in den hier als spezialisiert bezeichneten Studiengängen erweist sich das Labeling ebenfalls als different. Dies zeigt sich weiterhin vor allem in den klassischen Teildisziplinen (Sozialpädagogik, Rehabilitationspädagogik und Erwachsenenbildung), aber auch dem historisch jüngeren Bereich der Kindheitspädagogik. Hier machen die verwendeten pluralen Studienfachbezeichnungen deutlich, dass auch teildisziplinintern die Studiengangsreform im standortübergreifenden Sinne eher unkoordiniert verlaufen ist und keine übergreifende Orientierung in gemeinsamen Fachbezeichnungen zum Ausdruck kommt. Dies verweist - ebenso wie die differenten Gewichtungen der Studieninhalte - weniger auf teildisziplinäre Verselbstständigungsschritte, sondern rückt vielmehr die Frage ins Zentrum, inwieweit Profilbildungen und Alleinstellungsmerkmale sowohl disziplinäre als auch teildisziplinäre Grenzziehungen herausfordern. 
(5) Nach wie vor und mit zunehmender Deutlichkeit treten aufgrund der differenten Studienprogramme auch die bereits länger diskutierten Anschlussproblematiken im Übergang vom Bachelor in den Master hervor (schon Stisser et al. 2012; Grunert 2012; Grunert et al. 2016). Ist dies an ein und demselben Standort zumeist unproblematisch, wirft der Blick auf das Feld der Kombinationsmöglichkeiten im bundesdeutschen Studienspektrum erziehungswissenschaftlicher Hauptfachstudiengänge doch Fragen nach erschwerten Übergängen auf. Nicht nur die thematischen Spezialisierungen bereits auf der Bachelorebene, sondern auch die inhaltlichen Differenzen zwischen den Studiengängen verweisen auf Problematiken, die sich beim Übergang in den Master nicht nur für Studierende, sondern auch für Lehrende ergeben (Grunert/Ludwig 2019: 76) und die sich nicht zuletzt über intransparente Zulassungsordnungen potenzieren können. Auch hier stellt sich die Frage, inwieweit eine fachgesellschaftliche Thematisierung und Diskussion möglicher Übergangsregelungen künftige Gestaltungsprozesse begleiten kann.

\section{Literatur}

Ambrose, Don/VanTassel-Baska, Joyce/Coleman, Laurence J./Cross, Tracy L. (2010): Unified, insular, firmly policed, or fractured, porous, contested, gifted education? In: Journal for the Education of the Gifted 33, 4, S. 453-478.

DGfE (= Deutsche Gesellschaft für Erziehungswissenschaft) (2010): Kerncurriculum Erziehungswissenschaft. Empfehlungen der Deutschen Gesellschaft für Erziehungswissenschaft (2. erw. Aufl.). Opladen: Barbara Budrich.

Grunert, Cathleen (2012): Erziehungswissenschaft im Spiegel ihrer Studiengänge. Die Studienreform und ihre Folgen für die Disziplin. In: Zeitschrift für Erziehungswissenschaft 15, 2, S. 573-596.

Grunert, Cathleen/Ludwig, Katja (2016a): Disziplinen im Wandel? Erziehungswissenschaft und Soziologie im Bologna-Prozess. In: Zeitschrift für Pädagogik 62, 6, S. 886-908.

Grunert, Cathleen/Ludwig, Katja (2016b): Labels matter!? Erziehungswissenschaftliche Studiengänge in der Bologna-Reform. In: Bildung und Erziehung 69, 4, S. 449-466.

Grunert, Cathleen/Ludwig, Katja/Radhoff, Melanie/Ruberg, Christiane (2016): Studiengänge und Standorte. In: DGfE: Datenreport Erziehungswissenschaft 2016. Hrsg. v. Koller, H.-C./Faulstich-Wieland, H./Weishaupt, H./Züchner, I. Schriften der Deutschen Gesellschaft für Erziehungswissenschaft. Opladen u.a.: Verlag Barbara Budrich, S. 19-69.

Grunert, Cathleen/Ludwig, Katja (2019): »Was sind eigentlich die Ansprüche der Fachcommunity? « Anforderungen an die Fachgesellschaft DGfE in der Gestaltung erziehungswissenschaftlicher Studiengänge. In: Erziehungswissenschaft 30, 58, S. 73-79. 
Grunert, Cathleen/Rasch, Susann (2006): Gestufte erziehungswissenschaftliche Hochschulausbildung im internationalen Vergleich. In: DGfE: Datenreport Erziehungswissenschaft 2006. Hrsg. v. Kraul, M./Merkens, H./Tippelt, R. Schriften der Deutschen Gesellschaft für Erziehungswissenschaft. Wiesbaden: VS Verlag für Sozialwissenschaften, S. 15-40.

Horn, Klaus-Peter/Wigger, Lothar/Züchner, Ivo (2008): Standorte und Studiengänge. In: DGfE: Datenreport Erziehungswissenschaft 2008. Hrsg. v. Tillmann, K.-J./Rauschenbach, T./Tippelt, R./Weishaupt, H. Schriften der Deutschen Gesellschaft für Erziehungswissenschaft. Opladen u.a.: Verlag Barbara Budrich, S. 19-40.

HRK (=Hochschulrektorenkonferenz) (Hrsg.) (1998): Studienangebote deutscher Hochschulen WS 98/99. Bad Honnef: Verlag Karl Heinrich Bock.

Ludwig, Katja (2019): »Es war ein Ringen« - Zum Umgang mit disziplinärer Heterogenität im Kontext der Entwicklung erziehungswissenschaftlicher Hauptfachstudiengänge. In: Zeitschrift für Erziehungswissenschaft 22, 2, S. 461-479.

Ludwig, Katja/Grunert, Cathleen (2018): »Über die Früchte [...]« der Bologna-Reform. Rekonstruktionen zur Gestaltung erziehungswissenschaftlicher Hauptfachstudiengänge zwischen Disziplin und Hochschule. In: Vogel, K./Bers, C./Brauns, J./Hild, A./Stisser, A./Horn, K.-P. (Hrsg.): Wendungen und Windungen in der Erziehungswissenschaft. Empirische Studien. Bad Heilbrunn: Klinkhardt, S. 83-105.

Ludwig, Katja/Grunert, Cathleen (2020): Studiengangsentwicklung als Ausdruck disziplinärer Wandlungsprozesse? Erziehungswissenschaft zwischen fachgesellschaftlichen Empfehlungen und ausdifferenzierter Studiengangslandschaft. In: Binder, U./Meseth, W. (Hrsg.): Strukturwandel der Erziehungswissenschaft und der erziehungswissenschaftlichen Wissensproduktion. Bad Heilbrunn: Klinkhardt, i.E.

Rauschenbach, Thomas (1994): Ausbildung und Arbeitsmarkt für ErziehungswissenschaftlerInnen. In: Krüger, H.-H./Rauschenbach, T. (Hrsg.): Erziehungswissenschaft. Die Disziplin am Beginn einer neuen Epoche. Weinheim: Juventa, S. 275294.

Schubarth, Wilfried/Speck, Karsten (2014): Employability und Praxisbezüge im wissenschaftlichen Studium. HRK-Fachgutachten. https://www.hrk-nexus.de/fileadmin/ redaktion/hrk-nexus/07-Downloads/07-02-Publikationen/Fachgutachten_Employability-Praxisbezuege.pdf [Zugriff: 19.09.2019].

Schubarth, Wilfried/Speck, Karsten/Seidel, Andreas/Gottmann, Corinna/Kamm, Caroline/Krohn, Maud (Hrsg.) (2012): Studium nach Bologna: Praxisbezüge stärken?! Praktika als Brücke zwischen Hochschule und Arbeitsmarkt. Wiesbaden: Springer VS.

Stisser, Anna/Horn, Klaus-Peter/Züchner, Ivo/Ruberg, Christiane/Wigger, Lothar (2012): Studiengänge und Standorte. In: DGfE: Datenreport Erziehungswissenschaft 2012. Hrsg. v. Thole, W./Faulstich-Wieland, H./Horn, K.-P./Weishaupt, H./Züchner, I. Schriften der Deutschen Gesellschaft für Erziehungswissenschaft. Opladen u.a.: Verlag Barbara Budrich, S. 19-69.

Vogel, Peter (1994): Klassifikationsprobleme als Ausdruck des defizitären Charakters der Erziehungswissenschaft? In: Horn, K.-P./Wigger, L. (Hrsg.): Systematiken und 
Klassifikationen in der Erziehungswissenschaft. Weinheim: Deutscher Studienverlag, S. 371-387.

Züchner, Ivo (2012): Erziehungswissenschaftliche Studiengänge der Frühpädagogik an Fachhochschulen und Universitäten. In: DGfE: Datenreport Erziehungswissenschaft 2012. Hrsg. v. Thole, W./Faulstich-Wieland, H./Horn, K.-P./Weishaupt, H./ Züchner, I. Schriften der Deutschen Gesellschaft für Erziehungswissenschaft. Opladen u.a.: Verlag Barbara Budrich, S. 49-53. 


\title{
2 Studiengänge und Standorte der Lehrerinnen- und Lehrerbildung
}

\author{
Melanie Radhoff \& Christiane Ruberg
}

Mit dem Datenreport 2020 werden zum sechsten Mal Daten zu Studiengängen und Standorten im Bereich der Lehramtsstudiengänge an den deutschen Hochschulen und Universitäten dokumentiert. Die aktuelle Bestandsaufnahme nimmt insbesondere die Reform- und Entwicklungsverläufe im Lehramt in den Blick, die durch die Bologna-Reform vor zwanzig Jahren angestoßen wurden, und fragt nach Kontinuitäten und Neuorientierungen. Nachfolgend werden für das Lehramt die aktuellen Strukturvarianten, das Angebot an Studiengängen sowie Standorte, inklusive der Zentren für Lehrerinnen- und Lehrerbildung/Professional Schools of Education, dokumentiert und exemplarisch die universitären Praxisphasen im Rahmen des Grundschullehramtsstudiums aufgezeigt. Der aktuell hohe Bedarf an Lehrkräften, insbesondere für das Lehramt an berufsbildenden Schulen sowie für einzelne Fächerkombinationen insbesondere aus dem MINT-Bereich, hat zu einer Flexibilisierung der Zugänge in den Lehrberuf geführt. Am Beispiel des Quer- und Seiteneinstiegs werden auf der Basis aktueller Bedarfsprognosen alternative Wege in das Lehramt beschrieben und kritisch im Hinblick auf die Konsequenzen für die Lehrerinnen- und Lehrerprofessionalität diskutiert. Im Fazit wird die Frage aufgeworfen, welche Bedeutung den universitären bildungswissenschaftlichen Anteilen ${ }^{1}$ innerhalb der benannten Entwicklungstrends zukommt.

\subsection{Strukturvarianten in der Lehrerinnen- und Lehrerbildung}

In die vor zwanzig Jahren angestoßene Strukturreformdebatte innerhalb der Lehrerinnen- und Lehrerbildung scheint vorerst ein wenig Ruhe eingekehrt zu sein und die bereits im Datenreport 2016 (Grunert et al. 2016) dokumentierten Strukturvarianten sind weitgehend stabil. Während es zu Beginn des

1 Die Bezeichnungen der überfachlichen Studienanteile sind an den Hochschulen sehr unterschiedlich (z.B. erziehungswissenschaftliche Anteile, Bildungswissenschaft/en). Sofern nicht standortspezifisch ein Terminus vorgegeben ist, bietet es sich hier an "Bildungswissenschaften « als »curricularen Ordnungsbegriff « (vgl. Kunter et al. 2017: 40) zu verwenden; durchaus im kritischen Bewusstsein, dass "mit der Kennzeichnung auch theoretische und metatheoretische, methodische und methodologische Ansprüche und Selbstverständnisse, wissenschaftliche Praxen und soziale Netzwerke« verknüpft sind (Glaser/Keiner 2015: 9). 
Bologna-Prozesses noch eine offene Frage war, wie die Lehrerinnen- und Lehrerbildung »ihren adäquaten Platz in der sich abzeichnenden Umstellung der klassischen universitären Studien- und Abschlussstruktur (Diplom, Magister) auf das Modell der gestuften Studiengänge bzw. -abschlüsse (BA/MA) findet « (Terhart 2000: 95) und modularisierte Studiengänge als Modellversuche »Zunächst nur an einzelnen Standorten >erprobt « « wurden (Horn/Wigger/Züchner 2004: 26), hat sich mittlerweile eine klare Mehrheit der Bundesländer für die B/M-Variante entschieden.

Das Studium für berufliche Schulen wird in allen Bundesländern mit einem Master abgeschlossen, auch in Bayern, Hessen, Mecklenburg-Vorpommern, im Saarland und in Sachsen, wo die Studierenden aller anderen Schulformen die erste Phase der Ausbildung mit dem Staatsexamen beenden (vgl. Tabelle 2.1). Insgesamt gibt es innerhalb der Lehrerinnen- und Lehrerbildung 1.686 B-Studiengänge, $1.749 \mathrm{M}$-Studiengänge und 1.082 Studiengänge, die mit dem Staatsexamen abschließen (HRK 2018a: 12). In Sachsen-Anhalt und in Thüringen sind nach wie vor beide Strukturvarianten und damit beide Abschlüsse, je nach Universitätsstandort, möglich. Dieses Parallelangebot ist in den vergleichsweise kleinen Bundesländern bemerkenswert, denn bei der Frage, ob Staatsexamen oder akademischer Grad den Abschluss bilden, "geht es im Kern um die Entscheidung, wie vor dem Hintergrund der Autonomie der Hochschulen der Einfluss des Staates auf die erste Phase der Ausbildung beschaffen ist « (Oelkers 2018: 13f.). Das Nebeneinander von Staatsexamen und dem Bachelor/Master-Modell ist insbesondere aus studentischer Sicht kritisch zu bewerten, denn es steht dem »Anspruch an die Vereinfachung der studentischen Mobilität und der Vergleichbarkeit der Abschlüsse entgegen « (Mahrt/Wehage 2014: 14).

Die Deutsche Gesellschaft für Erziehungswissenschaft (DGfE) hat bereits 2004 ein Gesamtkonzept für die Lehrerinnen- und Lehrerbildung vorgelegt, das von einem »einheitlichen Strukturmodell für alle Lehramtsstudiengänge ausgeht « (DGfE 2004b: 1). Der Vorschlag umfasst ein dreijähriges polyvalentes Bachelorstudium sowie ein nach Schulstufen und -formen differenziertes zweijähriges Masterstudium. Von der empfohlenen einheitlichen Struktur der Studiengänge ist man allerdings nach wie vor weit entfernt, sodass die »ursprüngliche [...] Absicht, erst nach einem polyvalenten Bachelorstudium die definitive Entscheidung zum Lehrerinnen- und Lehrerberuf zu vollziehen und dann in einen professionsorientierten Master zu gehen " (Terhart 2014: 46), nicht flächendeckend eingelöst ist. Vielmehr lassen sich standortspezifisch bereits in früheren Datenreporten (vgl. Horn et al. 2004) dokumentierte polyvalente (z.B. Ruhr-Universität Bochum) als auch integrative Modelle (z.B. TU Dortmund) in der aktuellen Strukturlandschaft wiederfinden. ${ }^{2}$ Inhaltlich ist angesichts des uneinheitlichen Studienangebots mit dem 
»Kerncurriculum Erziehungswissenschaft« (DGfE 2004a: 1) ein Mindeststandard formuliert, der die »Mobilität zwischen den Studienstandorten sowie die Anschlussfähigkeit ergänzender und weiterbildender Studien « (ebd.) erleichtern soll. Die Kreditpunktanteile sind in den bildungswissenschaftlichen Studienelementen umfänglich und inhaltlich nach wie vor sehr heterogen. Da sich an diesem Befund seit dem Datenreport 2016 keine grundlegenden Veränderungen ergeben haben, wird auf eine erneute exemplarische Darstellung verzichtet.

Tabelle 2.1: Strukturvarianten

\begin{tabular}{|cccc|}
\hline Bundesland & B/M-Abschluss & $\begin{array}{l}\text { B/M-Abschluss und } \\
\text { Staatsexamen }\end{array}$ & $\begin{array}{l}\text { Staatsexamen und Lehrämter für } \\
\text { berufliche Schulen B/M-Abschluss }\end{array}$ \\
\hline BW & $\times$ & $\times$ \\
\hline BY & $\times$ & \\
\hline BE & $\times$ & $\times$ \\
\hline BB & $\times$ & $\times$ \\
\hline HB & $\times$ & \\
\hline HH & & \\
\hline HE & & \\
\hline MV & $\times$ & \\
\hline NI & $\times$ & \\
\hline NW & $\times$ & & \\
\hline RP & & \\
\hline SL & & \\
\hline SN & & \\
\hline ST & & \\
\hline SH & & \\
\hline TH & & \\
\hline Bachelor, M M Master & & \\
\hline Quelle: eigene Recherchen & & \\
\hline
\end{tabular}

\subsubsection{Ausbildungsdauer der Lehramtsstudiengänge}

Schon seit Jahren wird kritisiert, dass die Einteilung in sechs Lehramtstypen angesichts zentraler Entwicklungsprozesse im Bildungswesen, wie dem Ausbau von Ganztags- und Gemeinschaftsschulen sowie der zunehmenden Bedeutung kommunaler Bildungslandschaften im Zuge der Entwicklung eines inklusiven 
Schulsystems, nicht mehr zeitgemäß ist und die universitäre Lehrerbildung der Schulentwicklung in einzelnen Bundesländern hinterherhinkt (vgl. ErdsiekRave/John-Ohnesorg 2014). Ungeachtet dessen orientiert sich die Lehrerinnenund Lehrerbildung nach wie vor weitgehend traditionell an sechs Schulformen (Ausnahmen sind Berlin und Brandenburg) und für das sonderpädagogische Lehramt wurden grundständige Studiengänge in den letzten Jahren weiter ausgebaut (vgl. Grunert et al. 2016).

In Berlin, Brandenburg, Bremen, Hamburg, Niedersachen, NordrheinWestfalen, Schleswig-Holstein und Thüringen (Ausnahme Jena: Lehramt Typ 3) erstreckt sich ein Lehramtsstudium schulformunabhängig über zehn Semester. Die mit dieser Angleichung einhergehende Forderung nach gleicher Besoldung ist nach wie vor nicht umgesetzt, liegt jedoch zum Beispiel in Nordrhein-Westfalen mittlerweile als Gesetzentwurf vor. Angehende Lehrkräfte für das Lehramt an Gymnasien sowie an beruflichen Schulen studieren im bundesweiten Vergleich im Schnitt zwei Semester länger als ihre Kommilitoninnen und Kommilitonen für die Primarstufe bzw. Sekundarstufe I. Neben dem abgebildeten neun- bzw. zehnsemestrigen einschlägigen Lehramtsstudium haben sich für das Lehramt an beruflichen Schulen (Typ 5) die Qualifikations- und Ausbildungsvoraussetzungen angesichts des akuten Fachkräftemangels für diese Schulform flexibilisiert.

Auch wenn die zweite Phase nicht in die Verantwortung der Hochschulen fällt, lässt sich hier ebenfalls aktuell eine deutliche Flexibilisierung des zeitlichen Umfangs und der Gestaltung dokumentieren. Während traditionell das Erste Staatsexamen als Zugangsvoraussetzung gilt, können aktuell beim Querund Seiteneinstieg sowie im Vorbereitungsdienst an beruflichen Schulen weitere Qualifikationen angerechnet werden und die Dauer des Referendariats zum Teil erheblich verkürzen. Ein weiterer Trend sind unterschiedliche Teilzeitregelungen in den Bundesländern, sodass das Referendariat mittlerweile in zahlreichen Bundesländern, Ausnahmen sind aktuell noch Bayern und Sachsen-Anhalt (Stand 5/2019), aus familiären oder finanziellen Gründen auch mit reduzierter Arbeitszeit absolviert werden kann, was die Gesamtdauer entsprechend verlängert (vgl. GEW 2019). Mit der Einführung des Teilzeitreferendariats möchten die zuständigen Ministerien und Behörden die Ausbildung familienfreundlicher und damit in Zeiten akuten Lehrerinnen- und Lehrermangels attraktiver machen. 


\section{Tabelle 2.2: Gesamtausbildungsdauerder Lehramtsstudiengängein Semestern/ Regelstudienzeit}

\begin{tabular}{|c|c|c|c|c|c|c|c|}
\hline $\begin{array}{l}\frac{0}{c} \\
\frac{\pi}{\tilde{y}} \\
\frac{0}{0} \\
\frac{0}{3} \\
\infty\end{array}$ & Typ 1 & Typ 2 & Typ 3 & Typ 4 & Typ 5 & Typ 6 & $\begin{array}{l}\text { Referen- } \\
\text { dariat }\end{array}$ \\
\hline BW & $\begin{array}{c}8 \\
\text { (B-6; M-2) } \\
\text { Profilierung } \\
\text { Europalehr- } \\
\text { amt möglich } \\
\text { (PH Freiburg, } \\
\text { PH Karlsruhe) }\end{array}$ & & $\begin{array}{c}10 \\
\text { (B-6; M-4) } \\
\text { Profilierung } \\
\text { Europalehr- } \\
\text { amt möglich } \\
\text { (PH Freiburg, } \\
\text { PH Karlsruhe, } \\
\text { PH Ludwigs- } \\
\text { burg) }\end{array}$ & $\begin{array}{c}10 \\
(B-6 ; M-4) / \\
(B-8) \\
\text { Musik o. } \\
\text { Kunst }\end{array}$ & $\begin{array}{c}10 \\
\text { (B-6; M-4)/ } \\
\text { (B-7; M-3) } \\
\text { Kooperative } \\
\text { Studien- } \\
\text { gänge }\end{array}$ & $\begin{array}{c}10 \\
\text { (B-6; M-4) } \\
\text { grundständig/ } \\
\text { (M-4) Aufbau- } \\
\text { studium } \\
\text { (Vorausset- } \\
\text { zung: 1. o. } 2 . \\
\text { Staatsexamen } \\
\text { o. Lehramts- } \\
\text { master) }\end{array}$ & $\begin{array}{l}18 \text { bzw. } 30 \\
\text { Monate } \\
\text { (Teilzeit) }\end{array}$ \\
\hline BY & 7 & & 7 & $\begin{array}{c}9 \\
10 \text { (Psycholo- } \\
\text { gie/schulpsy- } \\
\text { chologischer } \\
\text { Schwerpunkt) }\end{array}$ & $\begin{array}{c}10 \\
(B-6 ; M-4)\end{array}$ & 9 & $\begin{array}{l}24 \text { Monate } \\
\text { (Teilzeitre- } \\
\text { gelung ab } \\
\text { 2019) }\end{array}$ \\
\hline BE & $\begin{array}{c}10 \\
(B-6 ; M-4)\end{array}$ & & $\begin{array}{r}1 \\
\text { (B-6; } \\
\text { Lehramt an Inte } \\
\text { darschulen un }\end{array}$ & $\begin{array}{l}\text { M-4) } \\
\text { grierten Sekun- } \\
\text { d Gymnasien }\end{array}$ & $\begin{array}{c}10 \\
(B-6 ; M-4)\end{array}$ & & $\begin{array}{l}18 \text { bzw. } 24 \\
\text { Monate } \\
\text { (Teilzeit) }\end{array}$ \\
\hline BB & $\begin{array}{c}10 \\
\text { (B-6; M-4) } \\
\text { Inklusionspäd. } \\
\text { Schwerpunkt } \\
\text { möglich }\end{array}$ & & $\begin{array}{r}1 \\
\text { (B-6; } \\
\text { Sekundarst }\end{array}$ & $\begin{array}{l}\text { M-4) } \\
\text { fen I und II }\end{array}$ & & $\begin{array}{c}10 \\
\text { (B-6; M-4) } \\
\text { geplant ab } \\
\text { WiSe 2020/21 }\end{array}$ & 12 Monate \\
\hline $\mathrm{HB}$ & $\begin{array}{c}10 \\
(B-6 ; M-4)\end{array}$ & & & $\begin{array}{c}10 \\
(B-6 ; M-4)\end{array}$ & $\begin{array}{c}10 \\
(B-6 ; M-4)\end{array}$ & $\begin{array}{c}10 \\
\text { (B-6; M-4) } \\
\text { Inklusive Päda- } \\
\text { gogik/Sonder- } \\
\text { pädagogik } \\
\end{array}$ & $\begin{array}{c}18 \text { Monate } \\
\text { (Teilzeitre- } \\
\text { gelung ab } \\
\text { 2019) }\end{array}$ \\
\hline $\mathrm{HH}$ & & $\begin{array}{c}10 \\
\text { (B-6; M-4) } \\
\text { (B-8) } \\
\text { Kunst, } \\
\text { Musik }\end{array}$ & & $\begin{array}{c}10 \\
\text { (B-6; M-4) } \\
\text { (B-8) Kunst, } \\
\text { Musik }\end{array}$ & $\begin{array}{c}10 \\
(B-6 ; M-4)\end{array}$ & $\begin{array}{c}10 \\
(B-6 ; M-4) \\
\text { (B-8) Kunst, } \\
\text { Musik }\end{array}$ & $\begin{array}{l}18 \text { bzw. } \\
24 \text { Monate } \\
\text { (Teilzeit) }\end{array}$ \\
\hline $\mathrm{HE}$ & 7 & & 7 & 9 & $\begin{array}{c}10 \\
(B-6 ; M-4)\end{array}$ & 9 & $\begin{array}{l}21 \text { bzw. } 27 \\
\text { oder } 33 \\
\text { Monate } \\
\text { (Teilzeit) }\end{array}$ \\
\hline MV & 9 & & 10 & 10 & $\begin{array}{c}\text { B-6 bis 7; } \\
\text { M-3 bis 4, ab- } \\
\text { hängig vom } \\
\text { Studienfach }\end{array}$ & 9 & $\begin{array}{c}18 \text { bzw. } 24 \\
\text { Monate bei } \\
\text { Doppel- } \\
\text { qualifika- } \\
\text { tion }^{1}\end{array}$ \\
\hline $\mathrm{NI}$ & $\begin{array}{c}10 \\
(B-6 ; M-4)\end{array}$ & & $\begin{array}{c}10 \\
(B-6 ; M-4)\end{array}$ & $\begin{array}{c}10 \\
\text { (B-6; M-4) } \\
\text { (B-8) Kunst, } \\
\text { Musik }\end{array}$ & $\begin{array}{c}10 \\
(B-6 ; M-4)\end{array}$ & $\begin{array}{c}10 \\
(B-6 ; M-4)\end{array}$ & 18 Monate \\
\hline
\end{tabular}




\begin{tabular}{|c|c|c|c|c|c|c|c|}
\hline 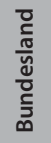 & Typ 1 & Typ 2 & Typ 3 & Typ 4 & Typ 5 & Typ 6 & $\begin{array}{l}\text { Referen- } \\
\text { dariat }\end{array}$ \\
\hline NW & $\begin{array}{c}10 \\
(B-6 ; M-4)\end{array}$ & & $\begin{array}{c}10 \\
(B-6 ; M-4)\end{array}$ & $\begin{array}{c}10 \\
(B-6 ; M-4)\end{array}$ & $\begin{array}{c}10 \\
(B-6 ; M-4)\end{array}$ & $\begin{array}{c}10 \\
(B-6 ; M-4)\end{array}$ & $\begin{array}{c}18 \text { bzw. } 24 \\
\text { Monate } \\
\text { (Teilzeit) }\end{array}$ \\
\hline $\mathrm{RP}$ & $\begin{array}{c}8 \\
(B-6 ; M-2)\end{array}$ & & $\begin{array}{c}9 \\
(B-6 ; M-3)\end{array}$ & $\begin{array}{c}10 \\
(B-6 ; M-4)\end{array}$ & $\begin{array}{c}10 \\
(B-6 ; M-4)\end{array}$ & $\begin{array}{c}9 \\
(B-6 ; M-3)\end{array}$ & 18 Monate \\
\hline SL & 8 & & 8 & 10 & $\begin{array}{c}10 \\
\text { Wirtschafts- } \\
\text { pädagogik } \\
(\mathrm{B}-6 ; \mathrm{M}-4) \\
\end{array}$ & & 18 Monate \\
\hline SN & 8 & & 9 & 10 & $\begin{array}{c}10 \\
12 \text { (in Kom- } \\
\text { bination mit } \\
\text { beruflicher } \\
\text { Ausbildung) }\end{array}$ & 10 & $\begin{array}{c}12 \text { bzw. } 24 \\
\text { Monate } \\
\text { (Teilzeit) }\end{array}$ \\
\hline ST & $\begin{array}{c}8 \\
\text { Halle }\end{array}$ & & $\begin{array}{c}8 \\
9 \text { Musik o. } \\
\text { Kunsterzie- } \\
\text { hung (Halle) } \\
10 \\
\text { (B-6; M-4) } \\
\text { (Magdeburg) }\end{array}$ & $\begin{array}{c}9 \\
10 \text { Musik o. } \\
\text { Kunsterzie- } \\
\text { hung (Halle) } \\
10 \\
\text { (B-6; M-4) } \\
\text { (Magdeburg) }\end{array}$ & $\begin{array}{c}10 \\
\text { (B-6; M-4) } \\
\text { (Magdeburg, } \\
\text { HS Merse- } \\
\text { burg) }\end{array}$ & $\begin{array}{c}9 \\
\text { (Halle) }\end{array}$ & $\begin{array}{l}16 \text { Monat } \\
\text { (keine } \\
\text { Teilzeitrege- } \\
\text { lung) }\end{array}$ \\
\hline $\mathrm{SH}$ & $\begin{array}{c}10 \\
(B-6 ; M-4)\end{array}$ & & $\begin{array}{c}10 \\
(B-6 ; M-4)\end{array}$ & $\begin{array}{c}10 \\
(\mathrm{~B}-6 ; \mathrm{M}-4) \\
(\mathrm{B}-8) \text { Musik }\end{array}$ & $\begin{array}{c}10 \\
(B-6 ; M-4)\end{array}$ & $\begin{array}{c}10 \\
(B-6 ; M-4)\end{array}$ & $\begin{array}{c}18 \text { bzw. } \\
24 \text { Monate } \\
\text { (Teilzeit) }\end{array}$ \\
\hline TH & $\begin{array}{c}10 \\
\text { (B-6; M-4) } \\
\text { (Erfurt) }\end{array}$ & & $\begin{array}{c}10 \\
\text { (B-6; M-4) } \\
\text { (Erfurt) } \\
9 \\
\text { (Jena) }\end{array}$ & $\begin{array}{c}10 \\
\text { (Jena) } \\
10 \\
\text { (B-6; M-4) } \\
\text { Musik }\end{array}$ & $\begin{array}{c}10 \\
\text { (B-6; M-4) } \\
\text { (Erfurt, Wei- } \\
\text { mar, Ilmenau, } \\
\text { Jena) }\end{array}$ & $\begin{array}{c}10 \\
(\mathrm{~B}-6 ; \mathrm{M}-4) \\
\text { (Erfurt) }\end{array}$ & $\begin{array}{l}24 \text { Monate } \\
\text { alle Schul- } \\
\text { formen } \\
\text { außer } \\
\text { Grund- } \\
\text { schulen (18 } \\
\text { Monate) }\end{array}$ \\
\hline
\end{tabular}

Typ 1 = Lehrämter der Grundschule bzw. Primarstufe, Typ 2 = Übergreifende Lehrämter der Primarstufe und aller oder einzelner Schularten der Sekundarstufe I, Typ 3 = Lehrämter für alle oder einzelne Schularten der Sekundarstufe I, Typ 4 = Lehrämter der Sekundarstufe II [allgemeinbildende Fächer] oder für das Gymnasium, Typ 5 = Lehrämter der Sekundarstufe II [berufliche Fächer] oder für die beruflichen Schulen, Typ 6 = Sonderpädagogische Lehrämter

$\mathrm{B} / \mathrm{M}=$ Bachelor-/Masterstudiengänge, ansonsten: Staatsexamensstudiengänge

Quelle: eigene Recherchen

1 Doppelqualifikation = bundesweit anerkannte Zweite Staatsprüfung für das Lehramt an Regionalen Schulen und die in Mecklenburg-Vorpommern gültige Unterrichtserlaubnis für die Tätigkeit an Grundschulen bzw. die bundesweit anerkannte Zweite Staatsprüfung für das Lehramt an Gymnasien und die in Mecklenburg-Vorpommern gültige Unterrichtserlaubnis für die Tätigkeit an Grundschulen oder Regionalen Schulen, s. auch: Merkblatt zum Vorbereitungsdienst (Ministerium für Bildung, Wissenschaft und Kultur MV). 


\subsubsection{Internationalisierung - ein Trend in der Lehrerinnen- und Lehrerbildung?}

Angesichts zunehmender sprachlicher und kultureller Vielfalt in Lerngruppen, wird die Ausbildung interkultureller Kompetenzen als eine zentrale Professionalisierungsaufgabe angehender Lehrkräfte markiert und die »Internationalisierung der Lehrerinnen- und Lehrerbildung erfährt daher wachsende Aufmerksamkeit« (HRK 2018b: 2). Im institutionell verankerten Ausbildungsangebot zeigt sich dies allerdings erst ansatzweise. In Baden-Württemberg haben Studierende mit nachweislich guten Englisch- bzw. Französischkenntnissen die Möglichkeit, das Studium für die Primarstufe (PH Freiburg, PH Karlsruhe) sowie für die Sekundarstufe I (PH Freiburg, PH Karlsruhe, PH Ludwigsburg) mit der Profilierung »Europalehramt« aufzunehmen (vgl. Tabelle 2.2). Das Studium zielt darauf ab, Fremdsprachen im Unterricht zielführend einzusetzen und produktiv mit der zunehmenden (sprachlichen) Heterogenität der Schülerschaft umzugehen. Es qualifiziert nicht dazu, in einem anderen europäischen Land zu unterrichten (vgl. PH Karlsruhe). In anderen Bundesländern gibt es an weiteren Hochschulen ebenfalls Bestrebungen, Internationalisierungsstrategien strukturell nachhaltig $\mathrm{zu}$ verstetigen. Beispiele hierfür sind das Zertifikat »Lehramt international « (LiCAU) an der Christian-Albrechts-Universität zu Kiel, das für eine zunehmende Interkulturalität vor Ort ausbildet oder der internationale LINT-Master der Universität Regensburg ${ }^{3}$, mit dem eine Qualifizierung für die Verbeamtung im französischen Staatsdienst einhergeht.

\subsection{Standorte Lehramtsstudiengänge}

Im ersten Datenreport 2000 wurden insgesamt 73 Hochschulen mit einem eigenständigen erziehungswissenschaftlichen Lehrangebot für das Lehramt Primarstufe, Sekundarstufe I und Sekundarstufe II systematisch gelistet (Rauschenbach/Züchner 2000: 27-29) und auf kooperierende Standorte - insbesondere Hochschulen für Musik und Hochschulen für Bildende Künste - verwiesen. Die Darstellung ist nun um das Lehramt an beruflichen Schulen, das sonderpädagogische Lehramt sowie um die Zentren für Lehrerinnen- und Lehrerbildung/Professional Schools of Education erweitert. Auch heute kann an insgesamt 73 Standorten ein Lehramtsstudium aufgenommen werden (vgl. Tabelle 2.3). Im Vergleich zum Jahr 2000 bietet die Universität Düsseldorf allerdings keine Lehramtsstudiengänge mehr an, die Hochschulen in Essen und Duisburg sind im Jahr 2003 fusioniert und mit der Fachhochschule Ostwestfalen-Lippe und Südwestfalen sind zwei (außeruniversitäre) Standorte hinzugekommen. Insbesondere durch Kooperationen mit Fachhochschulen und Hochschulen innerhalb des Studienangebots für das Lehramt an

3 Da diese Zusatz-Angebote über das grundständige Lehramtsstudium hinausgehen, sind sie in Tabelle 2.2 nicht aufgeführt. 
beruflichen Schulen hat sich das Spektrum in den letzten zwanzig Jahren weiter ausdifferenziert. Darüber hinaus hat sich das Angebot an Lehramtsstudiengängen nur im Detail standortspezifisch verändert: Zum Beispiel konnte an der TU Berlin im Jahr 2000 noch ein Studium für die Lehrämter Primarstufe sowie die Sekundarstufe I und II aufgenommen werden, wohingegen hier heute nur noch die Ausbildung für berufsbildende Schulen absolviert werden kann; in Kiel werden aktuell das Lehramt für die Sekundarstufe II und für berufsbildende Schulen angeboten, im Jahr 2000 war es auch noch das Studium für die Sekundarstufe I. Grundsätzlich kann heute in jedem Bundesland ein Lehramtsstudium für jede Schulform aufgenommen werden, Ausnahme ist das Saarland, in dem es kein Angebot für das sonderpädagogische Lehramt gibt. Im Datenreport 2016 (vgl. Grunert et al. 2016) war es ein besonderes Anliegen zu überprüfen, inwiefern sich Strukturreformen im Schulsystem, explizit die Umsetzung des Inklusionsgebots (Häcker/Walm 2015: 13) im Anschluss an die UN-Behindertenrechtskonvention im Jahr 2009 sowie der Trend zur Zweigliedrigkeit (vgl. Tillmann 2012) durch die Zusammenlegung von Schulformen in der Sekundartstufe I, in der Ausbildungsgestaltung widerspiegeln. Ergänzend zu den bereits 2016 erhobenen und dokumentierten Standorten mit einem sonderpädagogischen Lehramt, einem Kombinationsangebot von Regelschul- und sonderpädagogischem Lehramt oder integrierten Studiengängen an insgesamt 25 Standorten, wird der Ausbau sonderpädagogischer Studiengänge zum Beispiel mit dem für das Wintersemester 2020/21 geplanten Studiengang »Lehramt für Förderpädagogik « an der Universität Potsdam aktuell weiter vorangetrieben (vgl. Tabelle 2.3).

Darüber hinaus expandiert das Studienangebot für das Lehramt an beruflichen Schulen, indem Hochschulen und Fachhochschulen angesichts eines Mangels an Fachlehrerinnen und -lehrern in Kooperation mit lehrerinnen- und lehrerausbildenden Universitäten und Pädagogischen Hochschulen B-Studiengänge anbieten, die den nahtlosen Übergang in einen lehramtsspezifischen M-Studiengang ermöglichen. Auch wenn in den meisten Fällen die bildungswissenschaftlichen Module in das Masterstudium verlegt und damit an einer kooperierenden Universität studiert werden, gibt es mit den Fachhochschulen Ostwestfalen-Lippe und Südwestfalen erste Standorte, an denen diese Anteile bereits im Bachelor studiert werden (vgl. Websites der Fachhochschulen). Perspektivisch stellt sich die Frage, welche Auswirkungen die Ausdehnung des Studienangebots mit Lehramtsoption auf den Stellenwert der Lehrerinnen- und Lehrerbildung an den Hochschulen und insbesondere auf die Bedeutung der Bildungswissenschaften haben wird.

\subsubsection{Zentren für Lehrerinnen- und Lehrerbildung und Professional Schools of Education}

Insgesamt bilden die Lehramtsstudiengänge an Universitäten und Hochschulen mit 4.517 Studiengängen (WS 18/19) etwas weniger als ein Fünftel aller Stu- 
diengänge (vgl. HRK 2018a). Das breite Angebot von Lehramtsstudiengängen, einhergehend mit ungebrochen hohen Studierendenzahlen, korrespondiert an vielen Hochschulen nicht mit einem entsprechend hohen Status der Lehrerinnen- und Lehrerbildung, vielmehr erschwert die Beteiligung verschiedener Institutionen innerhalb der Universitäten »den Vertretern der Lehrerbildung, mit einer Stimme zu sprechen und hochschulintern ihre Interessen wahrzunehmen" (Husung 2014: 16). In diesem Sinne wird schon seit Jahren die »Residualstruktur « (Weiler 2010:18) und die damit einhergehende »organisatorische Zersplitterung " (Husung 2014: 16) der Lehrerinnen- und Lehrerbildung an den Hochschulen kritisiert. Um die auf verschiedene Fakultäten und Institute verteilte Lehrerinnen- und Lehrerbildung besser zu steuern und $\mathrm{zu}$ koordinieren, wurde im Zuge des Bologna-Prozesses die Gründung und der Ausbau von Zentren für Lehrerinnen- und Lehrerbildung gefördert. Zentrale Aufgaben sind neben der Beratung von Studierenden vor allem Planung, Organisation und Durchführung von Praxisphasen, Lehrerinnen- und Lehrerfortbildung sowie Lehr-Lern-Forschung. Die Zentren sind dabei entweder eher dienstleistungs- oder forschungsorientiert oder kombinieren beide Ansprüche miteinander (vgl. Weyand/Krämer 2010). Aus zahlreichen Zentren für Lehrerinnen- und Lehrerbildung, die mehrheitlich administrative und beratende Funktionen ausüben (vgl. Lindow/Shajek 2014), sind mittlerweile Professional Schools of Education (PSE) hervorgegangen (vgl. Tabelle 2.3). Diese definieren sich »als eigenständige Zentralinstitute quer zu den Fächern und Fakultäten. Sie werden somit zu einem nach innen und außen sichtbaren Überbau für die Lehrerbildung“ (ebd.: 39) mit dem erklärten Ziel einer besseren Verzahnung der verschiedenen Aufgabenbereiche der Lehrerinnen- und Lehrerbildung, insbesondere mit Blick auf die curriculare Ausgestaltung und eine professionsbezogene Forschung.

Bemerkenswert sind hier die Kooperationen einzelner Hochschulen untereinander. Zum Beispiel haben die Universität Stuttgart, die Pädagogische Hochschule Ludwigsburg, die Staatliche Akademie der Bildenden Künste Stuttgart, die Hochschule für Musik und Darstellende Kunst Stuttgart und die Universität Hohenheim im Rahmen einer Hochschulkooperation im Zuge der "Qualitätsoffensive Lehrerbildung" von Bund und Ländern eine gemeinsame "Professional School of Education" (PSE) gegründet. Darüber hinaus finden sich Kooperationen einzelner Zentren für Lehrerinnen- und Lehrerbildung bundesländerübergreifend vor allem in Projektkontexten. ${ }^{4}$

4 Beispielsweise kooperiert das Zentrum für Lehrerinnen- und Lehrerbildung der Universität Flensburg im Rahmen des Projekts "Schuladoption - Studierende machen Schule! «, bei dem ein Studierendenteam nach einer mehrwöchigen Vorbereitungsphase innerhalb des Praxisblocks für eine Woche eine Schule mit allen Aufgabenbereichen übernimmt, während das Kollegium gemeinsam eine Fortbildung besucht, mit der Pädagogischen Hochschule Weingarten. 
Tabelle 2.3: Standorte und Ausbildungsangebot

\begin{tabular}{|c|c|c|c|c|c|c|c|}
\hline Standort & Typ 1 & Typ 2 & Typ 3 & Typ 4 & Typ 5 & Typ 6 & ZfL/PSE \\
\hline \multicolumn{8}{|c|}{ Baden-Württemberg } \\
\hline Freiburg U & & & & $\times$ & & & $\begin{array}{c}\text { PSE } \\
\text { (in Koope- } \\
\text { ration mit } \\
\text { PH Frei- } \\
\text { burg) }\end{array}$ \\
\hline $\begin{array}{l}\text { Freiburg } \\
\mathrm{PH}\end{array}$ & $x$ & & $x$ & & $\begin{array}{l}\quad \times \\
\text { (in Koope- } \\
\text { ration mit } \\
\text { HS Offen- } \\
\text { burg) }\end{array}$ & & $\begin{array}{c}\text { PSE } \\
\text { (in Koope- } \\
\text { ration mit } \\
\text { U Freiburg) }\end{array}$ \\
\hline $\begin{array}{l}\text { Heidel- } \\
\text { berg U }\end{array}$ & & & & $\begin{array}{c}\quad \times \\
\text { (in Koope- } \\
\text { ration mit } \\
\text { HFJS } \\
\text { "Jüdische } \\
\text { Religions- } \\
\text { lehre«) }\end{array}$ & $\times$ & & $\begin{array}{c}\text { PSE } \\
\text { (in Koope- } \\
\text { ration mit } \\
\text { PH Heidel- } \\
\text { berg) }\end{array}$ \\
\hline $\begin{array}{l}\text { Heidel- } \\
\text { berg PH }\end{array}$ & $x$ & & $x$ & & $\begin{array}{c}\quad \times \\
\text { (in Koope- } \\
\text { ration mit } \\
\text { HS Mann- } \\
\quad \text { heim) }\end{array}$ & $x$ & $\begin{array}{c}\text { PSE } \\
\text { (in Koope- } \\
\text { ration mit } \\
\text { U Heidel- } \\
\text { berg) }\end{array}$ \\
\hline $\begin{array}{l}\text { Hohen- } \\
\text { heim U }\end{array}$ & & & & $x$ & $\times$ & & $\begin{array}{c}\text { PSE } \\
\text { (Hochschul- } \\
\text { koopera- } \\
\text { tion) }\end{array}$ \\
\hline $\begin{array}{l}\text { Karlsru- } \\
\text { he U }\end{array}$ & & & & $x$ & $\times$ & & $x$ \\
\hline $\begin{array}{l}\text { Karlsruhe } \\
\mathrm{PH}\end{array}$ & $x$ & & $x$ & & & & - \\
\hline $\begin{array}{l}\text { Konstanz } \\
\text { U }\end{array}$ & & & & $x$ & $x$ & & $x$ \\
\hline $\begin{array}{l}\text { Ludwigs- } \\
\text { burg PH }\end{array}$ & $\times$ & & $x$ & & $\begin{array}{c}\times \\
\text { (in Ko- } \\
\text { operation } \\
\text { mit HS } \\
\text { Esslingen) }\end{array}$ & $x$ & $\begin{array}{c}\text { PSE } \\
\text { (Hochschul- } \\
\text { koopera- } \\
\text { tion) }\end{array}$ \\
\hline $\begin{array}{l}\text { Mann- } \\
\text { heim U }\end{array}$ & & & & $x$ & $\times$ & & $x$ \\
\hline $\begin{array}{l}\text { Schwä- } \\
\text { bisch } \\
\text { Gmünd } \\
\text { PH }\end{array}$ & $x$ & & $x$ & & $\begin{array}{c}\quad \times \\
\text { (in Koope- } \\
\text { ration mit } \\
\text { HS Aalen) } \\
\end{array}$ & & - \\
\hline $\begin{array}{l}\text { Stuttgart } \\
U\end{array}$ & & & & $\times$ & $x$ & & $\begin{array}{c}\text { PSE } \\
\text { (Hochschul- } \\
\text { koopera- } \\
\text { tion) }\end{array}$ \\
\hline
\end{tabular}


Studiengänge und Standorte der Lehrerinnen- und Lehrerbildung

\begin{tabular}{|c|c|c|c|c|c|c|c|}
\hline Standort & Typ 1 & Typ 2 & Typ 3 & Typ 4 & Typ 5 & Typ 6 & ZfL/PSE \\
\hline $\begin{array}{l}\text { Tübingen } \\
U\end{array}$ & & & & $\times$ & $\times$ & & $x$ \\
\hline UIm U & & & & $\times$ & & & $\times$ \\
\hline $\begin{array}{l}\text { Weingar- } \\
\text { ten } \mathrm{PH}\end{array}$ & $x$ & & $x$ & & $\begin{array}{c}\times \\
\text { (in Koopera- } \\
\text { tion mit HS } \\
\text { Ravens- } \\
\text { burg-Wein- } \\
\text { garten) } \\
\end{array}$ & & $\times$ \\
\hline $\begin{array}{l}\text { Kunst- } \\
\text { und } \\
\text { Musik- } \\
\text { hoch- } \\
\text { schulen' }\end{array}$ & & & & $\begin{array}{c}\times \\
\text { (in Koope- } \\
\text { ration mit } \\
\text { Partneruni- } \\
\text { versitäten) }\end{array}$ & & & $\begin{array}{c}\text { PSE } \\
\text { (Hoch- } \\
\text { schulko- } \\
\text { operation }\end{array}$ \\
\hline \multicolumn{8}{|c|}{ Bayern } \\
\hline $\begin{array}{l}\text { Augsburg } \\
U\end{array}$ & $x$ & & $x$ & $x$ & & & $x$ \\
\hline $\begin{array}{l}\text { Bamberg } \\
\mathrm{U}\end{array}$ & $\times$ & & $x$ & $x$ & $\times$ & & $\times$ \\
\hline $\begin{array}{l}\text { Bayreuth } \\
\text { U }\end{array}$ & & & $\times$ & & $\times$ & & $\times$ \\
\hline $\begin{array}{l}\text { Eichstätt- } \\
\text { Ingolstadt } \\
\mathrm{KU}\end{array}$ & $\times$ & & $\times$ & $\times$ & & & $x$ \\
\hline $\begin{array}{l}\text { Erlangen- } \\
\text { Nürnberg } \\
\text { U }\end{array}$ & $\times$ & & $\times$ & $\begin{array}{c}\text { (in Ko- } \\
\text { operation } \\
\text { mit AdBK } \\
\text { München) }\end{array}$ & $x$ & & $\times$ \\
\hline $\begin{array}{l}\text { München } \\
\text { U }\end{array}$ & $\begin{array}{c}\times \\
\text { (in Koope- } \\
\text { ration mit } \\
\text { HMTM } \\
\text { München) }\end{array}$ & & $\begin{array}{c}\times \\
\text { (in Koope- } \\
\text { ration mit } \\
\text { HMTM } \\
\text { München) }\end{array}$ & $\begin{array}{c}\times \\
\text { (in Koope- } \\
\text { ration mit } \\
\text { HMTM } \\
\text { München) }\end{array}$ & $\times$ & $x$ & $\times$ \\
\hline $\begin{array}{l}\text { München } \\
\text { TU }\end{array}$ & $\begin{array}{c}\times \\
\text { (in Koope- } \\
\text { ration mit } \\
\text { HMTM } \\
\text { München) }\end{array}$ & & $\begin{array}{c}\times \\
\text { (in Ko- } \\
\text { operation } \\
\text { mit HMTM } \\
\text { München) }\end{array}$ & $\begin{array}{c}\times \\
\text { (in Koope- } \\
\text { ration mit } \\
\text { HMTM } \\
\text { München) }\end{array}$ & $\times$ & & PSE \\
\hline Passau U & $\times$ & & $\times$ & $\times$ & & & $\times$ \\
\hline $\begin{array}{l}\text { Regens- } \\
\text { burg U }\end{array}$ & $\times$ & & $\times$ & $\times$ & & & $x$ \\
\hline $\begin{array}{l}\text { Würzburg } \\
\text { U }\end{array}$ & $\begin{array}{c}\times \\
\text { (in Ko- } \\
\text { operation } \\
\text { mit HfM } \\
\text { Würzburg) }\end{array}$ & & $\begin{array}{c}\times \\
\text { (in Ko- } \\
\text { operation } \\
\text { mit HfM } \\
\text { Würzburg) }\end{array}$ & $\begin{array}{c}\times \\
\text { (in Ko- } \\
\text { operation } \\
\text { mit HfM } \\
\text { Würzburg) }\end{array}$ & & $x$ & PSE \\
\hline
\end{tabular}




\begin{tabular}{|c|c|c|c|c|c|c|c|}
\hline Standort & Typ 1 & Typ 2 & Typ 3 & Typ 4 & Typ 5 & Typ 6 & ZfL/PSE \\
\hline \multicolumn{8}{|c|}{ Berlin } \\
\hline Berlin FU & $\begin{array}{l}\quad \times \\
\text { (in Koope- } \\
\text { ration mit } \\
\text { UdK Berlin) }\end{array}$ & & & $\begin{array}{c}\times \\
\text { (ISS/Gym) } \\
\text { (in Koope- } \\
\text { ration mit } \\
\text { UdK Berlin) }\end{array}$ & & $x$ & $x$ \\
\hline Berlin HU & $\times$ & & & $\begin{array}{c}\times \\
\text { (ISS/Gym) } \\
\text { (in Koope- } \\
\text { ration mit } \\
\text { UdK Berlin) }\end{array}$ & $\times$ & $\begin{array}{c}\times \\
\text { (Kombi B } \\
\text { mit } \\
\text { Lehramts- } \\
\text { option) }\end{array}$ & PSE \\
\hline Berlin TU & & & & $\begin{array}{c}\times \\
\text { (ISS/Gym) } \\
\text { (in Koope- } \\
\text { ration mit } \\
\text { UdK Berlin) }\end{array}$ & $\times$ & & $\times$ \\
\hline \multicolumn{8}{|c|}{ Brandenburg } \\
\hline $\begin{array}{l}\text { Potsdam } \\
\text { U }\end{array}$ & $x$ & & & $\times$ & & $\begin{array}{c}\times \\
(a b \text { WS } \\
20 / 21)\end{array}$ & $\times$ \\
\hline \multicolumn{8}{|c|}{ Bremen } \\
\hline Bremen U & $\times$ & & & $\times$ & $\times$ & $\times$ & $\times$ \\
\hline \multicolumn{8}{|c|}{ Hamburg } \\
\hline $\begin{array}{l}\text { Hamburg } \\
\text { U }\end{array}$ & & $x$ & & $x$ & $\begin{array}{c}\times \\
\text { (in Koopera- } \\
\text { tion mit TU } \\
\text { Hamburg- } \\
\text { Harburg) }\end{array}$ & $x$ & $x$ \\
\hline \multicolumn{8}{|c|}{ Hessen } \\
\hline $\begin{array}{l}\text { Darmstadt } \\
\text { TU }\end{array}$ & & & & $\times$ & $x$ & & $x$ \\
\hline $\begin{array}{l}\text { Frankfurt } \\
\text { U }\end{array}$ & $x$ & & $\times$ & $\times$ & & $\times$ & $\times$ \\
\hline Gießen U & $\times$ & & $\times$ & $\times$ & $\times$ & $\times$ & $\times$ \\
\hline Kassel U & $\times$ & & $\times$ & $\times$ & $\times$ & & $\times$ \\
\hline $\begin{array}{l}\text { Marburg } \\
\mathrm{U}\end{array}$ & & & & $\times$ & & & $\times$ \\
\hline \multicolumn{8}{|c|}{ Mecklenburg-Vorpommern } \\
\hline $\begin{array}{l}\text { Greifswald } \\
\text { U }\end{array}$ & & & $\times$ & $\times$ & & & $\times$ \\
\hline
\end{tabular}




\begin{tabular}{|c|c|c|c|c|c|c|c|}
\hline Standort & Typ 1 & Typ 2 & Typ 3 & Typ 4 & Typ 5 & Typ 6 & ZfL/PSE \\
\hline Rostock U & $\begin{array}{c}\times \\
\text { (in Ko- } \\
\text { operation } \\
\text { mit HMT } \\
\text { Rostock) }\end{array}$ & & $\begin{array}{c}\times \\
\text { (in Ko- } \\
\text { operation } \\
\text { mit HMT } \\
\text { Rostock) }\end{array}$ & $\begin{array}{c}\times \\
\text { (in Koope- } \\
\text { ration mit } \\
\text { HMT } \\
\text { Rostock) }\end{array}$ & $\begin{array}{c}\times \\
\text { (in Ko- } \\
\text { operation } \\
\text { mit HS } \\
\text { Neubran- } \\
\text { denburg) }\end{array}$ & $\begin{array}{c}\times \\
\text { (in Ko- } \\
\text { operation } \\
\text { mit HMT } \\
\text { Rostock) }\end{array}$ & $x$ \\
\hline \multicolumn{8}{|c|}{ Niedersachsen } \\
\hline $\begin{array}{l}\text { Braun- } \\
\text { schweig } \\
\text { TU }\end{array}$ & $\times$ & & $\times$ & $\begin{array}{l}\quad \times \\
\text { (in Koope- } \\
\text { ration mit } \\
\text { HBK Braun- } \\
\text { schweig) }\end{array}$ & & & $\times$ \\
\hline $\begin{array}{l}\text { Göttin- } \\
\text { gen U }\end{array}$ & & & & $\times$ & $\times$ & & $\times$ \\
\hline $\begin{array}{l}\text { Hanno- } \\
\text { ver U }\end{array}$ & & & & $\times$ & $\times$ & $\times$ & PSE \\
\hline $\begin{array}{l}\text { Hildes- } \\
\text { heim U }\end{array}$ & $\times$ & & $\times$ & & & & $\times$ \\
\hline $\begin{array}{l}\text { Lüneburg } \\
\text { U }\end{array}$ & $x$ & & $x$ & & & & $\times$ \\
\hline $\begin{array}{l}\text { Olden- } \\
\text { burg U }\end{array}$ & $\times$ & & $x$ & $\times$ & $\times$ & $x$ & $x$ \\
\hline $\begin{array}{l}\text { Osna- } \\
\text { brück U }\end{array}$ & $x$ & & $x$ & $\times$ & $\times$ & & $\times$ \\
\hline Vechta U & $\times$ & & $x$ & & & & $\times$ \\
\hline \multicolumn{8}{|c|}{ Nordrhein-Westfalen ${ }^{2}$} \\
\hline Aachen U & & & & $x$ & $\begin{array}{c}\times \\
\text { (in Koope- } \\
\text { ration mit } \\
\text { FH Aachen, } \\
\text { TH Köln } \\
\text { und HS Nie- } \\
\text { derrhein) }\end{array}$ & & $x$ \\
\hline $\begin{array}{l}\text { Bielefeld } \\
\text { U }\end{array}$ & $\times$ & & $\times$ & $\times$ & & $\times$ & PSE \\
\hline Bochum U & & & & $\times$ & & & PSE \\
\hline Bonn U & & & & & $x$ & & $x$ \\
\hline $\begin{array}{l}\text { Dortmund } \\
\text { TU }\end{array}$ & $\times$ & & $x$ & $\times$ & $\begin{array}{c}\times \\
\text { (in Koope- } \\
\text { ration mit } \\
\text { FH Dort- } \\
\text { mund) }\end{array}$ & $x$ & $x$ \\
\hline $\begin{array}{l}\text { Duisburg- } \\
\text { Essen U }\end{array}$ & $\begin{array}{c}\times \\
\text { (in Ko- } \\
\text { operation } \\
\text { mit UdK } \\
\text { Folkwang) }\end{array}$ & & $\begin{array}{c}\times \\
\text { (in Ko- } \\
\text { operation } \\
\text { mit UdK } \\
\text { Folkwang) }\end{array}$ & $\begin{array}{c}\times \\
\text { (in Ko- } \\
\text { operation } \\
\text { mit UdK } \\
\text { Folkwang) }\end{array}$ & $x$ & & $\times$ \\
\hline
\end{tabular}




\begin{tabular}{|c|c|c|c|c|c|c|c|}
\hline Standort & Typ 1 & Typ 2 & Typ 3 & Typ 4 & Typ 5 & Typ 6 & ZfL/PSE \\
\hline Köln U & $\begin{array}{c}\quad \times \\
\text { (in Koope- } \\
\text { ration mit } \\
\text { DSHS Köln) }\end{array}$ & & $\begin{array}{c}\quad \times \\
\text { (in Koope- } \\
\text { ration mit } \\
\text { DSHS Köln) }\end{array}$ & $\begin{array}{c}\times \\
\text { (in Koope- } \\
\text { ration mit } \\
\text { HfMT Köln u. } \\
\text { DSHS Köln) } \\
\end{array}$ & $\begin{array}{c}\times \\
\text { (in Koope- } \\
\text { ration mit } \\
\text { HfMT Köln u. } \\
\text { DSHS Köln) } \\
\end{array}$ & $\begin{array}{c}\times \\
\text { (in Koope- } \\
\text { ration mit } \\
\text { DSHS Köln) }\end{array}$ & $\times$ \\
\hline Münster U & $\times$ & & $x$ & $\times$ & $\begin{array}{c}\times \\
\text { (in Ko- } \\
\text { operation } \\
\text { mit FH } \\
\text { Münster) } \\
\end{array}$ & & $\times$ \\
\hline $\begin{array}{l}\text { Ostwest- } \\
\text { falen- } \\
\text { Lippe } \mathrm{FH}^{3}\end{array}$ & & & & & $x^{*}$ & & - \\
\hline $\begin{array}{l}\text { Paderborn } \\
\text { U }\end{array}$ & $\times$ & & $\times$ & $\times$ & $\begin{array}{c}\times \\
\text { (in Koopera- } \\
\text { tion mit FH } \\
\text { Bielefeld u. } \\
\text { HS Hamm- } \\
\text { Lippstadt) }\end{array}$ & $\times$ & PSE \\
\hline Siegen U & $\begin{array}{c}\times \\
\text { (in Koope- } \\
\text { ration mit } \\
\text { DSHS Köln) }\end{array}$ & & $\begin{array}{c}\times \\
\text { (in Koope- } \\
\text { ration mit } \\
\text { DSHS Köln) }\end{array}$ & $\begin{array}{c} \\
\times \\
\text { (in Koope- } \\
\text { ration mit } \\
\text { DSHS Köln) }\end{array}$ & $\begin{array}{c}\times \\
\text { (in Koopera- } \\
\text { tion mit HS } \\
\text { Bonn-Rhein- } \\
\text { Sieg, FH } \\
\text { Dortmund, } \\
\text { FH Südwest- } \\
\text { falen, TH } \\
\text { Köln und } \\
\text { DSHS Köln) }\end{array}$ & $\begin{array}{c}\times \\
\text { (in Koope- } \\
\text { ration mit } \\
\text { DSHS Köln) }\end{array}$ & $x$ \\
\hline $\begin{array}{l}\text { Südwest- } \\
\text { falen } \mathrm{FH}^{4}\end{array}$ & & & & & $x^{*}$ & & - \\
\hline $\begin{array}{l}\text { Wupper- } \\
\text { tal U }\end{array}$ & $\times$ & & $\times$ & $\times$ & $\begin{array}{c}\quad \times \\
\text { (in Koope- } \\
\text { ration mit } \\
\text { HS Bochum } \\
\text { und Westfä- } \\
\text { lischen } \mathrm{HS}^{5} \text { ) }\end{array}$ & $\times$ & PSE \\
\hline \multicolumn{8}{|c|}{ Rheinland-Pfalz } \\
\hline $\begin{array}{l}\text { Kaisers- } \\
\text { lautern U }\end{array}$ & & & $\times$ & $\times$ & $\times$ & & $\times$ \\
\hline $\begin{array}{l}\text { Koblenz- } \\
\text { Landau U }\end{array}$ & $x$ & & $x$ & $x$ & $\begin{array}{c}\times \\
\text { (Standort } \\
\text { Koblenz) }\end{array}$ & $\begin{array}{c}\times \\
\text { (Standort } \\
\text { Landau) }\end{array}$ & $x$ \\
\hline Mainz U & & & & $\times$ & & & $\times$ \\
\hline Trier U & & & $x$ & $\times$ & & & $\times$ \\
\hline \multicolumn{8}{|c|}{ Saarland } \\
\hline $\begin{array}{l}\text { Saarbrücken } \\
\text { u. Hom- } \\
\text { burg U }\end{array}$ & $x$ & & $x$ & $\times$ & $\times$ & & $x$ \\
\hline
\end{tabular}




\begin{tabular}{|c|c|c|c|c|c|c|c|}
\hline Standort & Typ 1 & Typ 2 & Typ 3 & Typ 4 & Typ 5 & Typ 6 & ZfL/PSE \\
\hline \multicolumn{8}{|c|}{ Sachsen } \\
\hline $\begin{array}{l}\text { Chem- } \\
\text { nitz U }\end{array}$ & $\times$ & & & & & & $\times$ \\
\hline $\begin{array}{l}\text { Dresden } \\
\text { TU }\end{array}$ & $\times$ & & $\times$ & $\times$ & $\times$ & & $\times$ \\
\hline Leipzig U & $\times$ & & $x$ & $\times$ & & $\times$ & $\times$ \\
\hline \multicolumn{8}{|c|}{ Sachsen-Anhalt } \\
\hline $\begin{array}{l}\text { Halle-Wit- } \\
\text { tenberg U }\end{array}$ & $\times$ & & $\times$ & $\times$ & & $\times$ & $\times$ \\
\hline $\begin{array}{l}\text { Magde- } \\
\text { burg U }\end{array}$ & & & $\times$ & $x$ & $\begin{array}{c}\times \\
\text { (ab WS } \\
\text { 19/20 BA: } \\
\text { Ingenieurs- } \\
\text { pädagogik in } \\
\text { Kooperation } \\
\text { mit HS Harz) }\end{array}$ & & $\times$ \\
\hline \multicolumn{8}{|c|}{ Schleswig-Holstein } \\
\hline $\begin{array}{l}\text { Flensburg } \\
\text { U }\end{array}$ & $x$ & & $x$ & & $\times$ & $\times$ & $\times$ \\
\hline Kiel U & & & & $\times$ & $\times$ & & $\times$ \\
\hline \multicolumn{8}{|c|}{ Thüringen } \\
\hline Erfurt U & $\times$ & & $\times$ & & $\times$ & $\times$ & PSE \\
\hline Jena U & & & $\times$ & $\times$ & & & $\times$ \\
\hline
\end{tabular}

AdBK $=$ Akademie der Bildenden Künste, DSHS $=$ Deutsche Sporthochschule; FH = Fachhochschule, FU = Freie Universität, HfJS = Hochschule für Jüdische Studien, HfM= Hochschule für Musik, HMT = Hochschule für Musik und Theater, HMTM = Hochschule für Musik und Theater München, HS = Hochschule, HU = Humboldt-Universität, KU = Katholische Universität, $\mathrm{PH}=$ Pädagogische Hochschule, PSE = Professional School of Education, $\mathrm{TH}=$ Technische Hochschule, $\mathrm{TU}=$ Technische Universität, $\mathrm{U}=$ Universität, UdK = Universität der Künste, ZfL = Zentrum für Lehrerinnen- und Lehrerbildung

Quelle: KMK 2017 und eigene Recherchen Stand 5/2019

1 Kunst- und Musikhochschulen kooperieren mit mehreren Hochschulen und Universitäten.

2 Lehramtstyp 5 an den Standorten FH Aachen, FH Bielefeld, FH Dortmund, FH Münster, HS Bochum, HS Bonn-Rhein-Sieg, HS Hamm-Lippstadt, HS Niederrhein, Westfälische HS und TH Köln: kein lehramtsbezogener Studiengang, sondern ein Fachbachelor mit der Option, zusätzliche Module zu absolvieren, die den Zugang zu einem Lehramtsmaster-Studiengang an kooperierenden Universitäten eröffnen können.

3 Bachelor Lehramt ist ohne Kooperation mit einer anderen Universität möglich, für den Master muss jedoch das Studium an der U Paderborn fortgeführt werden (s. auch TH Ostwestfalen-Lippe).

4 Bildungswissenschaftliche Module für den Bachelor werden an der FH absolviert, Weiterführung des Studiums (M) an der U Paderborn, U Siegen oder U Wuppertal, (s. auch FH Südwestfalen).

5 Westfälische Hochschulen: Standorte in Ahaus, Bocholt, Gelsenkirchen, Recklinghausen. 


\subsubsection{Die universitären Praxisphasen}

Verbindliche Praxisphasen sind heute feste Elemente in jedem Lehramtsstudium. Im aktuellen Diskurs um die schulpraktischen Studien geht es vorrangig um die Qualität der konkreten Gestaltung und Begleitung durch die Universität (vgl. Biederbeck/Rothland 2018; Grassmé/Biermann/Gläser-Zikuda 2018). Anknüpfend an die vorausgegangenen Datenreporte, in denen die verbindlichen Praxisanteile für das Lehramt der Sekundarstufe I (2012) sowie für das gymnasiale Lehramt (2016) dokumentiert wurden, werden im Folgenden die schulpraktischen Anteile für das Lehramt an Grundschulen zusammengefasst. Die diversen Bezeichnungen und Angaben für die Praxisphasen wurden bundeslandspezifisch aus Praktikumsordnungen, Modulhandbüchern und Websites übernommen (Stand 05/2019). Sie geben einen Hinweis darauf, dass neben Dauer und Verortung im Studium sowie unterschiedlicher Anteile von Leistungspunkten und Kreditpunkten auch die Intention eines Praktikums durchaus variiert. Die Vergleichbarkeit ist erschwert durch unterschiedliche Zeitangaben (Stunden, Tage, Monate), variierende Kreditpunktanteile sowie die Zuordnung zu Bildungswissenschaften oder zu den Fächern. Gemeinsam ist allen Bundesländern ein im Bachelor bzw. in den ersten Studiensemestern vorgesehenes Orientierungspraktikum/Schulpraktikum.

Die DGfE empfiehlt in ihrem »Gesamtkonzept für die Lehrerbildung« (DGfE 2004b) im Sinne der Polyvalenz im Bachelor darüber hinaus ein verbindliches berufsorientierendes Praktikum im außerschulischen pädagogischen Bereich. Die Mehrheit der Bundesländer mit Bachelorstudiengängen sieht dies vor, Ausnahmen sind Berlin, Hamburg, Bremen und Schleswig-Holstein. Die Dauer der Praxisphasen unterscheidet sich bundeslandspezifisch insbesondere in den Masterstudiengängen bzw. höheren Semestern stark: Während Studierende in Nordrhein-Westfalen ein fünfmonatiges Praxissemester absolvieren, gehen Kommilitoninnen und Kommilitonen in MecklenburgVorpommern sechs Wochen, in Baden-Württemberg (mindestens) drei Wochen oder in Rheinland-Pfalz fünfzehn Tage in die Schulpraxis.

So lässt sich auch für die Praxisphasen resümieren, dass jedes Bundesland und zum Teil einzelne Standorte eine »individuelle Lösung erarbeitet [haben, d.V.], angepasst an die Ressourcen und die Gegebenheiten vor Ort, dies aber eben pragmatisch, nicht systematisch« (Böttcher/Blasberg 2015: 21). 


\section{Tabelle 2.4: Praxisphasen Grundschullehramt}

\begin{tabular}{|c|c|}
\hline $\begin{array}{l}\text { Bundes- } \\
\text { land }\end{array}$ & Typ 1 und Typ 2 \\
\hline BW & $\begin{array}{l}\text { B: begleitetes Orientierungspraktikum: } 3 \text { Wochen (5-9 ECTS; bis 3. Semester), integriertes } \\
\text { Semesterpraktikum: mind. } 12 \text { Wochen (15-30 ETCS; ab 3. Semester), ggf. weitere Praktika } \\
\text { (fachabhängig) } \\
\text { M: Professionalisierungspraktikum: mind. } 3 \text { Wochen (3-6 ECTS) }\end{array}$ \\
\hline BY & $\begin{array}{l}\text { Orientierungspraktikum: 3-4 Wochen (i.d.R. vor Studienbeginn), Betriebspraktikum: } \\
8 \text { Wochen (i.d.R. vor Studienbeginn), pädagogisch-didaktisches Schulpraktikum: } 5 \text { Wochen } \\
\text { (6 ECTS; studienbegl.), einsemestriges fachdidaktisches Praktikum: } 1 \text { Tag/Woche (5 ECTS; } \\
\text { studienbegl.), einsemestriges grundschuldidaktisches Praktikum: } 1 \text { Tag/Woche }\end{array}$ \\
\hline BE & $\begin{array}{l}\text { B: berufsfelderschließendes Praktikum/Orientierungspraktikum: } 6 \text { Wochen } \\
\text { M: Praxissemester ( } 30 \text { ECTS; Unterrichtspraktika in drei Fächern und ein Lernforschungsprojekt) }\end{array}$ \\
\hline BB & $\begin{array}{l}\text { B: integriertes Eignungspraktikum/Orientierungspraktikum: Praktikum in pädagogisch- } \\
\text { psychologischen Handlungsfeldern außerhalb der Schule: } 30 \text { Std., zwei fachdidaktische } \\
\text { Tagespraktika: je } 30 \text { U-Std. } \\
\text { M: Praxissemester: mind. } 16 \text { Wochen, parallel dazu findet das psychodiagnostische Praktikum } \\
\text { statt }\end{array}$ \\
\hline $\mathrm{HB}$ & $\begin{array}{l}\text { B: Orientierungspraktikum: } 6 \text { Wochen }(6 \mathrm{CP}) \text {, zwei praxisorientierte Elemente: je } 2-3 \text { Wochen }(3 \mathrm{CP}) \\
\text { M: Praxissemester: ein Schulhalbjahr }(27 \mathrm{CP})\end{array}$ \\
\hline $\mathrm{HH}$ & $\begin{array}{l}\text { B: Integriertes Schulpraktikum: } 4 \text { Wochen ( } 8 \text { LP) } \\
\text { M: Kernpraktikum I (Mittelstufe) und Kernpraktikum II (Primarstufe): je 10-15 semesterbeglei- } \\
\text { tende Praxistage und } 20 \text { bzw. } 25 \text { Tage Blockpraktikum (je } 15 \text { LP) }\end{array}$ \\
\hline $\mathrm{HE}$ & $\begin{array}{l}\text { Orientierungspraktikum: } 4 \text { Wochen (i.d.R. vor Studienbeginn; Voraussetzung für SPS I), } \\
\text { Betriebspraktikum: } 8 \text { Wochen ODER Praxissemester im 3./4. Semester (s.u.), Schulpraktische } \\
\text { Studien I und II: je } 5 \text { Wochen Blockpraktikum inkl. Unterrichtsversuche (14 CP; studienbegl.), } \\
\text { Praxissemester: } 250 \text { Std. in } 4 \text { Wochen Blockphase und 11-12 Wochen Langphase (nur an der } \\
\text { Universität Kassel; } 30 \text { CP; studienbegl.) }\end{array}$ \\
\hline MV & $\begin{array}{l}\text { Sozialpraktikum: } 85 \text { Std. in } 3 \text { Wochen (3 LP; außerschulisch), Orientierungspraktikum I } \\
\text { (Hospitationspraktikum an Grundschulen): } 3 \text { Wochen (3 LP; 3.-5. Semester), Orientierungs- } \\
\text { praktikum II (Profillinienpraktikum in Kita oder 5./6. Klasse): } 3 \text { Wochen (3 LP; 5.-8. Semester), } \\
\text { Hauptpraktikum (schulisch): } 6 \text { Wochen (6 LP; 6.-8. Semester) }\end{array}$ \\
\hline $\mathrm{NI}$ & $\begin{array}{l}\text { B: Vorbereitende Studien I: } 1 \text { Tag/Woche (semesterbegleitend), Vorbereitende Studien II: } \\
3 \text { Wochen, allgemeines Schulpraktikum: } 6 \text { Wochen (Braunschweig) ODER Schulpraktische } \\
\text { Studien I und II: (7 LP), allgemeines Schulpraktikum: } 4 \text { Wochen (4 LP), außerschulisches } \\
\text { Praktikum: } 4 \text { Wochen (Hildesheim; } 4 \text { LP) ODER Sozial- und Betriebspraktikum, Schulpraktische } \\
\text { Studien I: mind. } 3 \text { Wochen (5 CP), Schulpraktische Studien II: mind. } 4 \text { Wochen (Lüneburg; } 5 \text { CP) } \\
\text { ODER Orientierungspraktikum: } 4 \text { Wochen (6 CP), allgemeines Schulpraktikum: } 6 \text { Wochen } \\
\text { (Oldenburg, Vechta; } 9 \text { CP) ODER Betriebs- oder Sozialpraktikum: } 4 \text { Wochen, allgemeines } \\
\text { Schulpraktikum: } 5 \text { Wochen (Osnabrück) } \\
\text { M: Praxisblock (GHR 300): } 18 \text { Wochen an } 3 \text { Tagen/Woche }\end{array}$ \\
\hline
\end{tabular}

B: Eignungs- und Orientierungspraktikum: 5 Wochen (3-7 LP), Berufsfeldpraktikum: mind.

NW $\quad 4$ Wochen (3-6 LP)

M: Praxissemester: mind. 5 Monate (12-25 LP) 


\begin{tabular}{|c|c|}
\hline $\begin{array}{l}\text { Bundes- } \\
\text { land }\end{array}$ & Typ 1 und Typ 2 \\
\hline $\mathrm{RP}$ & $\begin{array}{l}\text { B: zwei orientierende Praktika: je } 15 \text { Tage (je } 3 \text { CP; davon kann eines an einem außerschuli- } \\
\text { schen Lern- oder Ausbildungsort - »Betriebspraktikum« - durchgeführt werden; ein Prak- } \\
\text { tikum muss an einer Schule mit inklusivem Unterricht durchgeführt werden), vertiefendes } \\
\text { Praktikum: } 15 \text { Tage ( } 4 \text { CP) } \\
\text { M: vertiefendes Praktikum: } 15 \text { Tage ( } 4 \text { CP) }\end{array}$ \\
\hline SL & $\begin{array}{l}\text { Orientierungspraktikum: } 5 \text { Wochen, zwei Schulpraktika: je } 4 \text { Wochen (je } 9 \text { ECTS), zwei semes- } \\
\text { terbegleitende fachdidaktische Praktika: je } 1 \text { Tag/Woche (je } 7 \text { ECTS) }\end{array}$ \\
\hline SN & $\begin{array}{l}\text { Schulpraktische Studien I: } 3 \text { Std./Woche semesterbegleitendes, bildungswissenschaftliches } \\
\text { Orientierungspraktikum und } 2 \text { Wochen Kita-Blockpraktikum, Schulpraktische Studien II } \\
\text { (schulpraktische Übung): } 3 \text { Std./Woche (semesterbegleitend), Schulpraktische Studien III } \\
\text { (schulpraktische Übung): } 3 \text { Std./Woche (semesterbegleitend), Schulpraktische Studien IV } \\
\text { (fachdidaktisches Blockpraktikum): } 4 \text { Wochen, Schulpraktische Studien V (fachdidaktisches } \\
\text { Blockpraktikum): } 4 \text { Wochen (Chemnitz) ODER: Grundpraktikum: } 60 \text { Std. (2 LP), drei Blockprak- } \\
\text { tika: } 100 \text { bzw. } 150 \text { Std. (je } 5 \text { LP), zwei schulpraktische Übungen: } 120 \text { Std. (je } 4 \text { LP) (Dresden) } \\
\text { ODER Schulpraktische Studien I: } 4 \text { Wochen Blockpraktikum, Schulpraktische Studien II } \\
\text { (schulpraktische Übung): } 1 \text { Tag/Woche semesterbegleitend, Schulpraktische Studien III: } 1 \text { Tag/ } \\
\text { Woche semesterbegleitend, Schulpraktische Studien IV: (fachdidaktisches Blockpraktikum): } \\
4 \text { Wochen, Schulpraktische Studien V: (fachdidaktisches Blockpraktikum): } 4 \text { Wochen (Leipzig) }\end{array}$ \\
\hline ST & $\begin{array}{l}\text { Beobachtungspraktikum: } 2 \text { Wochen (10 LP), Schulpraktikum I mit schulpraktischen Übungen: } \\
4 \text { Wochen (10 LP), Schulpraktikum II: } 4 \text { Wochen ( } 5 \text { LP), außerunterrichtliches pädagogisches } \\
\text { Praktikum: } 2 \text { Wochen ( } 5 \text { LP) }\end{array}$ \\
\hline $\mathrm{SH}$ & $\begin{array}{l}\text { B: Orientierungspraktikum: } 6 \text { Wochen, fachdidaktisches Praktikum: } 3 \text { Wochen (10 LP) } \\
\text { M: Praxissemester: } 10 \text { Wochen (15 LP) }\end{array}$ \\
\hline TH & $\begin{array}{l}\text { B: berufsorientierendes Schulpraktikum: } 80 \text { Std. (3 LP), vorbereitendes Schulpraktikum: } \\
2 \text { Wochen ( } 3 \text { LP) } \\
\text { M: komplexes Schulpraktikum: } 15 \text { Wochen ( } 30 \text { LP) }\end{array}$ \\
\hline \multicolumn{2}{|c|}{$\mathrm{B}=$ Bachelor, $\mathrm{M}=$ Master } \\
\hline Quell & K 2017, eigene Recherchen \\
\hline
\end{tabular}

\subsection{Bedarfsprognosen}

Durch Migration und steigende Geburtenraten ergibt sich über alle Schulformen hinweg ein deutlicher Zusatzbedarf an neuen Stellen für Lehrerinnen und Lehrer. Eine zudem steigende Altersstruktur derzeit beschäftigter Lehrkräfte führt kumulativ zu weiterem Ersatzbedarf auf dem Stellenmarkt (vgl. Brandt 2017). Im Gegensatz zu früheren Prognosen über Einstellungsbedarfe von Lehrerinnen und Lehrern, die insbesondere in den westlichen Bundesländern von einer überwiegenden Sättigung des Arbeitsmarktes ausgegangen sind, stellt sich die Situation nach aktuellen Prognosen der KMK (2018) anders dar: In den westdeutschen Bundesländern ist demnach das vermutete durchschnittliche jährliche Überangebot deutlich abgeschwächt und die schon seit Längerem be- 
obachtete Bedarfsunterdeckung in den ostdeutschen Bundesländern deutlich gestiegen. Im gesamtdeutschen Durchschnitt konnten Klemm und Zorn (2018) allein für die Grundschule herausstellen, dass bis zum Schuljahr 2030/2031 ein Ersatzbedarf von 81.000 vollzeitäquivalenten Lehrerinnen- und Lehrerstellen aufgrund des altersbedingten Ausscheidens von Grundschullehrkräften notwendig wäre. Hinzu kommen weitere notwendige Stellen (1.300 im Schuljahr 2020/21 und eine weitere Erhöhung auf 3.800 im Schuljahr 2025/2026) für steigende Schülerinnen- und Schülerzahlen sowie Stellen für zusätzliche pädagogische Aufgaben, wie beispielsweise den Ausbau von Ganztagsschulen. Klemm und Zorn (2018) gehen in ihren Prognosen davon aus, dass in den nächsten Jahren im Schnitt etwa 7.000 Absolventinnen und Absolventen mit einer Lehrbefähigung für die Grundschule in den Schuldienst eingestellt werden können. Zwischen den Schuljahren 2020/2021 und 2025/2026, ein Abschnitt, der einen besonders hohen Bedarf markiert, werden jedoch mindesten 9.100 jährlich eingestellte Lehrkräfte erforderlich, um den Erhalt des Unterrichts ermöglichen zu können. Diese Bedarfsprognosen skizzieren ein prekäres Bild der Schullandschaft, das insbesondere vor dem Hintergrund geforderter pädagogischer Maßnahmen, wie beispielsweise die Entwicklung und Umsetzung didaktischer Programme zur Leseförderung auf Grundlage der IGLU-Studie (vgl. Bremerich-Vos/Stahns/Hußmann/Schurig 2017), Fragen der Umsetzbarkeit aufwirft. Auch die KMK (2018) konstatiert, dass bis 2030 nicht genug ausgebildete Lehrkräfte zur Verfügung stehen, um den Bedarf zu decken.

Da mit dem Vorbereitungsdienst eine Gesamtausbildungsdauer von sechs bis sieben Jahren kalkuliert werden muss, sind Ausweitungen der Studienplätze für die kurzfristigen Bedarfsprognosen wenig zielführend, sodass kurzfristige Maßnahmen erforderlich sind (vgl. Klemm/Zorn 2017; 2018).

\subsubsection{Neue Wege ins Lehramt: der Quer- und Seiteneinstieg}

Um den derzeitigen Mangel an Lehrkräften kurzfristig beheben zu können, wird in den Bundesländern auf sogenannte Quer- und/oder Seiteneinsteigerinnen und -einsteiger zurückgegriffen. Diese haben kein Lehramtsstudium und auch kein Referendariat absolviert, "aber [sie verfügen] über eine berufliche bzw. akademische Qualifikation [...], die als Zugang zum Lehrerberuf anerkannt werden kann" (Amendt 2018: o.S.). Die begriffliche Differenzierung in Quer- und Seiteneinstiege suggeriert eine klare Trennung zwischen den qualifikationsbezogenen Anforderungsprofilen und Tätigkeitsfeldern im Schuldienst. Tabelle A3 (siehe Anhang) listet die Situation in den einzelnen Bundesländern auf und zeigt, dass die Profile und Zugangsvoraussetzungen von Quer- und Seiteneinstiegsmöglichkeiten zwischen den Bundesländern individuell sehr unterschiedlich ausgestaltet sind. Das oftmals aufgeführte Differenzierungskriterium, der Quereinstieg erfordere das zweite Staatsexamen, 
der Seiteneinstieg hingegen nicht und führe auch nicht zur Verbeamtung (vgl. ebd.), kann so nicht bestätigt werden. Auch beim Seiteneinstieg muss in vielen Fällen das Referendariat absolviert werden, nicht selten werden zudem äquivalente Qualifizierungsmöglichkeiten angeboten, die dann eine Gleichstellung mit dem Abschluss des zweiten Staatsexamens ermöglichen. Somit ist es sowohl im Quer- als auch im Seiteneinstieg möglich und sogar wahrscheinlich, verbeamtet zu werden. Jedoch ist es nicht selten so, dass Quer- und Seiteneinsteigerinnen und -einsteiger in niedrigeren Entgeltgruppen eingestuft werden, insbesondere in den Fällen, in denen keine Verbeamtung vorliegt. Neben den weitestgehend sicheren Perspektiven, die das Lehramt bietet, droht mit den unterschiedlichen Besoldungen damit eine Verschärfung der Spaltung des Kollegiums in zwei Klassen, die sich aktuell bereits im Kontext von Inklusion, beispielsweise anhand der unterschiedlichen Bezahlung von Grundschul- und sonderpädagogischen Lehrkräften, offenbart.

In den meisten Bundesländern und für die meisten Schulformen muss ein zweites Unterrichtsfach aus der vorhandenen Qualifikation abgeleitet werden, um vergleichbare Einsatzvoraussetzungen zu haben wie die Kolleginnen und Kollegen, die ein Lehramtsstudium absolviert haben. Die meisten Standorte bieten jedoch auch eine - in der Regel berufsbegleitende (in nur wenigen Ausnahmefällen vorab) - Nachqualifizierung für das zweite Fach innerhalb von ein bis zwei Jahren an, wenn dieses zweite Fach nicht oder nicht in ausreichendem Umfang vorhanden ist. Ausnahmen stellen hier meist die berufsbildenden Schulen dar, bei denen auch die Qualifikation in einem Fach ausreichend sein kann. Die Dokumentenanalyse hinsichtlich der Rolle der lehrerinnen- und lehrerbildenden Universitäten und damit der Bildungswissenschaften zeigt, dass die Universitäten nur in wenigen Fällen (in Bremen wird das zweite Fach an der Universität studiert, in Brandenburg wird die Qualifikation durch ein An-Institut der Universität Potsdam angeboten) an dieser Nachqualifizierung beteiligt sind, sondern vielmehr die Zentren für schulpraktische Lehrerausbildung (ZfsL), die üblicherweise die zweite Phase der Lehrerinnen- und Lehrerbildung verantworten. Durch diese Vermengung der ersten und zweiten Phase der Lehrerinnen- und Lehrerbildung drängt sich der Verdacht auf, dass es sich eher um eine "Light-Version « der pädagogischen und didaktischen Qualifizierung angehender Lehrkräfte handelt, die weitreichende Konsequenzen für die Professionalisierung des Lehrberufs haben könnte. Universitäten werden insbesondere dann eingebunden, wenn Bewerberinnen und Bewerber in Bezug auf das Abschlussniveau nicht hinreichend qualifiziert sind, wie es beispielsweise bei Fachhochschul- oder Bachelor-Abschlüssen der Fall ist. In diesen Fällen kann ein Master of Education berufsbegleitend nachstudiert werden. Hier ist der Berufseinstieg so organisiert, dass neben der Unterrichtspraxis Studienblöcke eingeplant sind, in denen das zweite Fach begleitend absolviert wird (in Baden-Württemberg, Nordrhein-Westfalen und Sachsen). Nach dieser Qualifikation werden die Absolventinnen und Absolventen mit denjenigen Studieren- 
den gleichgestellt, die das Fach grundständig über das gesamte Lehramtsstudium hinweg studiert haben. In vielen Bundesländern wird darauf verwiesen, dass Quer- und Seiteneinsteigerinnen und -einsteiger einen erhöhten Bedarf an Unterstützungsmaßnahmen benötigen und erfahrene Lehrkräfte daher als Mentorinnen und Mentoren unterstützen sollen. Fraglich ist jedoch, inwiefern im schulischen Alltag zeitliche Ressourcen für die erhöhten Unterstützungsbedarfe vorhanden sind und nicht eher Belastungen für beide Seiten resultieren (vgl. Priboschek 2016).

Auch ein Blick auf die Schulformen offenbart eine große Divergenz: In allen Bundesländern, die für den Quer- und Seiteneinstieg konkrete Schulformen benennen, werden für die berufsbildenden Schulen besonders hohe Einstellungsbedarfe markiert. So werben zwölf Bundesländer um Quer- und Seiteneinstiege für das Lehramt an beruflichen Schulen, was sich auch in dem stets sehr ausdifferenziert ausgeschriebenen Fächerkanon widerspiegelt. Dagegen bieten nur sieben Bundesländer diese Option für das Grundschullehramt an und für Förderschulen bzw. den sonderpädagogischen Bereich ist dies lediglich in fünf Bundesländern möglich, obwohl beide Bereiche einen hohen Einstellungsbedarf zu verzeichnen haben (vgl. Millmann 2018). In Nordrhein-Westfalen wird beispielsweise der Seiteneinstieg für das Grundschullehramt mit den Fächern Deutsch und Mathematik ausgeschlossen, mit der Begründung, dass fachliche und didaktische Anforderungen zu hoch seien und mit einem grundständigen Lehramtsstudium erworben werden müssen (vgl. Tabelle A3 im Anhang). Dies deckt sich mit der kritischen Perspektive, die der Einstellung von Quer- und Seiteneinsteigerinnen und -einsteigern entgegengebracht wird. So stellt sich oftmals die Frage, ob diese Praxis »die pädagogische Qualität der Schulen gefährdet« (Priboschek 2016: 38).

Als Zugangsvoraussetzung sowohl in den Quer- als auch Seiteneinstieg werden i.d.R. universitäre Masterabschlüsse oder äquivalente Abschlüsse vorausgesetzt. Ein Master von einer Fachhochschule wird häufig auch akzeptiert, hier besteht jedoch die Voraussetzung, dass es sich um einen akkreditierten Studiengang handelt. Diplom- oder Bachelorabschlüsse der Fachhochschule werden beispielsweise in Mecklenburg-Vorpommern akzeptiert, wenn mindestens eine zusätzliche dreijährige Berufserfahrung vorhanden ist. Zusammen mit der Tatsache, dass Fachhochschul-Abschlüsse (Bachelor oder Diplom) den Weg zu einem Master of Education ermöglichen, deutet dies Bestrebungen der Durchlässigkeit zwischen Fachhochschule und universitärer Bildung bzw. bestimmten beruflichen Positionen an. In Schleswig-Holstein steht Bachelor- oder Diplomabsolventinnen und -absolventen mit zweijähriger Berufserfahrung insbesondere an berufsbildenden Schulen sogar der Weg des Direkteinstiegs in den Schuldienst bereit, wenn keine hinreichende Zahl an Bewerbungen von fachlich höher qualifizierten Personen vorliegt. Doch auch ohne Hochschulabschluss gibt es mögliche Wege in das Lehramt: In Baden-Württemberg besteht für beruflich Qualifizierte ohne Hochschulabschluss die Möglichkeit, in mu- 
sisch-technischen Fächern für das Lehramt an Haupt-, Werkreal-, Real- und Gemeinschaftsschulen sowie als Fachlehrkraft für Sonderpädagogik ausgebildet zu werden. Auch in diesen Fällen erfolgt die Qualifizierung für die berufliche Praxis nicht an Universitäten, sondern am pädagogischen Fachseminar. An fast allen Standorten werden für den Quer- und/oder Seiteneinstieg pädagogische Qualifizierungsmaßnahmen verpflichtend angeboten, diese variieren jedoch auch in Form und Länge und werden z.T. im Block berufsvorbereitend angeboten (z.B. eine Woche in Berlin und ein Monat in Brandenburg) und in einigen Fällen zusätzlich berufsbegleitend. Die Varianz resultiert hier z.T. auch aus der angestrebten Schulform (in Grundschulen wird der höchste pädagogische Qualifizierungsbedarf gesehen) und nach vorhandenem Abschluss.

Es stellt sich die Frage, ob diese zeitlich und inhaltlich dichten pädagogischen Nachqualifizierungen einem grundständigen bildungswissenschaftlichen Anteil im Lehramtsstudium gleichgestellt werden können, das beispielsweise mit begleiteten Praktika viel Raum für Reflexionen der Lehrerinnen- und Lehrerrolle und wichtige geschützte Erfahrungsräume bietet (vgl. Radhoff/Ruberg/Wieckert 2019). Für Quer- und Seiteneinsteigerinnen und -einsteiger wird aufgrund des fehlenden bzw. auf eine "Light-Version" reduzierten bildungswissenschaftlichen Anteils in der Berufsvorbereitung nicht selten ein " Praxisschock«" (Priboschek 2016: 39) befürchtet, auch wenn dieser nicht zwingend eintreten muss. So zeigen Studien, dass zwar häufig die Selbstwirksamkeit im Hinblick auf Schülerinnen- und Schülermotivation sowie Klassenführung im Laufe der ersten Praxiserfahrungen sinkt, dies jedoch »für den ersten längeren Praxiskontakt mit schulischen Handlungsfeldern nicht ungewöhnlich « (Abs/ Eckert/Anderson-Park 2016: 9) ist. Zudem steigt beim Quer- und Seiteneinstieg gleichzeitig häufig die wahrgenommene Unterrichtskompetenz analog zum Wissensaufbau (vgl. ebd.). Einschränkend bleibt an dieser Stelle der Hinweis, dass in diesen Studien häufig eine spezifische Stichprobe zugrunde liegt: So haben in der zitierten Studie vier Fünftel der Quer- und Seiteneinsteigenden Erfahrungen in der Arbeit mit Kindern und Jugendlichen (vgl. ebd.). Für Quer- und Seiteneinsteigerinnen und -einsteiger, die diese Erfahrungen nicht vorweisen können, bleibt die Frage, ob ebenso positive Erfahrungen beim Berufseinstieg zu erwarten sind. Befunde weisen darauf hin, »dass hoch ausgeprägtes bildungswissenschaftliches Wissen den Einstieg in die Berufspraxis erleichtert und das berufliche Handeln positiv beeinflussen kann« (Kunter et al. 2017: 37), was erneut die Frage der geeigneten Qualifizierung aufwirft.

Festzuhalten bleibt, dass Quer- und Seiteneinsteigerinnen und -einsteiger im Gegensatz zu Absolventinnen und Absolventen aus Lehramtsstudiengängen stets nachrangig eingestellt werden und immer nur dann, wenn es keine ausreichende Zahl an qualifizierten Bewerbungen gibt. In Sachsen, SachsenAnhalt und Thüringen werden die Schulformen und Fächer jedes Schuljahr erneut bedarfsorientiert ausgeschrieben, sodass potenzielle Bewerberinnen und Bewerber im Vorfeld nicht wissen können, ob ein Quer- oder Seiteneinstieg 
möglich sein wird. Wenn der Einstieg aber einmal geglückt ist und die Personen im System angekommen sind und das zweite Staatsexamen erworben haben oder ihre Qualifikation diesem gleichgestellt wurde, wird danach keine Differenzierung mehr zu Lehramtsabsolventinnen und -absolventen vorgenommen. Auch ohne zweites Staatsexamen sind Entfristungen die Regel und auch spätere Verbeamtungen nicht unüblich. Es stellt sich die Frage, ob die verstetigten Stellen der Quer- und Seiteneinsteigerinnen und -einsteiger zu einem ähnlich gelagerten Phänomen wie in den 1960er und 1970er Jahren werden, in denen sich der Mangel zum Überfluss von Lehrerinnen und Lehrern verwandelte (vgl. Criblez 2017).

\subsection{Ausblick: Kontinuitäten und Diskontinuitäten}

In die insgesamt etwas leiser gewordene Diskussion um Strukturen hat sich in den letzten Jahren ein verstärkter Diskurs um Qualitätsfragen gemischt (vgl. Schubarth 2017; Oelkers 2018). So gibt es zahlreiche Bestrebungen zur Profilierung der Lehrerinnen- und Lehrerbildung durch eine bessere Verzahnung von Fachwissenschaft, Fachdidaktik und Bildungswissenschaften sowie durch die Stärkung der Zusammenarbeit der drei Phasen der Lehrerinnen- und Lehrerbildung, ergänzt durch Bemühungen um qualitätshaltige Praxisbezüge im Lehramtsstudium. Welche Rolle den Zentren für Lehrerinnen- und Lehrerbildung/Professional Schools of Education für die konkrete Ausgestaltung zukommt oder der mit mehr als 500 Millionen Euro durch Bund und Länder geförderten "Qualitätsoffensive Lehrerbildung ", die mit einem vergleichbaren Themenportfolio Reformen in der Lehrerinnen- und Lehrerbildung anstoßen möchte (vgl. BMBF o.J.), wird zurzeit evaluiert. Im Zwischenbericht zur Evaluation der "Qualitätsoffensive Lehrerbildung « wird ein stärkerer Austausch zwischen den Bundesländern angesichts der bislang länderspezifischen Strukturentwicklung an den Hochschulen empfohlen, um »den bundeslandübergreifenden Transfer jenseits der Hochschulen [zu, d.V.] verstärken « (Brümmer et al. 2018: 126, Herv. i.O.). Es bleibt abzuwarten, ob im Zuge einer nachhaltigen und standortunabhängigen Verstetigung der Programmziele erneut eine Strukturdiskussion entfacht. Die erkennbaren Bemühungen um Qualitätssicherung, die im hochkomplexen Aufgabenspektrum von Lehrkräften begründet sind, werden allerdings gleichzeitig durch arbeitsmarktbedingte Faktoren unterlaufen (vgl. Terhart 2014). So haben sich beim Quer- und Seiteneinstieg durch sehr heterogene Zugangsbedingungen in den Lehrberuf die Ausgangslagen weiter ausdifferenziert und zunehmend von einer Standardisierung entfernt. Tenorth (2018) betont in der durch den Lehrerinnen- und Lehrermangel begründeten aktuellen Dynamik das produktive Potenzial, denn »die klassisch gewordene triadische Sequenz von Hochschulstudium, Berufseinführung durch die Pro- 
fession und Ausbildung der Expertise im Berufsalltag ist sachlich unbestritten notwendig, aber ihre organisatorische Umsetzung ist sicherlich nicht in Erz gegossen. Am Quereinstieg und mit der Berufspraxis könnte man prüfen, welche Alternativen für Ausbildungsorte, Formen und Akteure bestehen, auch für die zweite Phase oder die Universitäten « (Tenorth 2018, o.S.). Bemerkenswert ist, dass beim Quer- und Seiteneinstieg die pädagogischen Ausbildungsanteile zum einen stark verkürzt sind und zum anderen in den Institutionen der zweiten Phase verantwortet werden. Damit geht eine stark am Schulalltag orientierte inhaltliche Ausrichtung einher, die sich zum Teil vorrangig von bildungswissenschaftlichen Curricula im traditionellen Lehramtsstudium unterscheidet. Welche Folgen diese Entwicklungen für den Stellenwert der universitären Bildungswissenschaften hat, wird sich in den nächsten Jahren zeigen. Mögliche Entwicklungsperspektiven für die universitären Bildungswissenschaften zeigen sich aktuell bereits anhand eines zunehmend ausdifferenzierten Angebots von studienbegleitenden Zusatzqualifikationen sowie berufsbegleitenden Aufbaustudiengängen (vgl. Radhoff/Ruberg 2018).

\section{Literatur}

Abs, Hermann Josef/Eckert, Thomas/Anderson-Park, Eva (2016): Effektivität der Qualifizierung von Teach First Fellows. Abschlussbericht zur summativen Evaluation der Sommerakademie von Teach First Deutschland. Frankfurt a.M.: Gesellschaft zur Förderung Pädagogischer Forschung.

Amendt, Jürgen (2018): Quer- und Seiteneinstieg. Notlösung wird zum Dauerzustand. https://www.gew.de/aktuelles/detailseite/neuigkeiten/notloesung-wird-zum-dauerzustand/ [Zugriff: 9.9.2019].

Biederbeck, Ina/Rothland, Martin (2018): Praxisphasen in der Lehrerbildung im Fokus der Bildungsforschung - Zur Einführung. In: Rothland, M./Biederbeck, I. (Hrsg.): Praxisphasen in der Lehrerbildung im Fokus der Bildungsforschung. Münster: Waxmann, S. 7-14.

Böttcher, Wolfang/Blasberg, Sina (2015): Strategisch aufgestellt und professionell organisiert? Eine explorative Studie zu Strukturen und Status der Lehrerbildung. Hrsg. v. Deutsche Telekom Stiftung. https://www.hrk.de/fileadmin/_migrated/content_uploads/Studie_Querstrukturen.pdf [Zugriff: 16.5.2019].

Brandt, Eberhard (2017): Neuordnung der Lehrer_innenbildung. Politische Auseinandersetzung im Zeichen des Nachwuchsmangels. In: Forum Wissenschaft 34, 4, S. 16-19.

Bremerich-Vos, Albert/Stahns, Ruven/Hußmann, Anke/Schurig, Michael (2017): Förderung von Leseflüssigkeit und Leseverstehen im Leseunterricht. In: Hußmann, A./Wendt, H./Bos, W./Bremerich-Vos, A./Kasper, D./Lankes, E.-M./McElvany, N./ Stubbe, T./Valtin, R. (Hrsg.): IGLU 2016. Lesekompetenz von Grundschulkindern in Deutschland im internationalen Vergleich. Münster: Waxmann, S. 279-296. 
Brümmer, Felix/Durdel, Anja/Fischer-Münnich, Christiane/Fittkau, Jacob/Weiger, Wiebke (2018): Qualitätsoffensive Lehrerbildung. Zwischenbericht der Evaluation. In: Ramboll Management Consulting GmbH (Hrsg.): Qualitätsoffensive Lehrerbildung. Zwischenbericht der Evaluation. https://www.gwk-bonn.de/fileadmin/ Redaktion/Dokumente/Papers/Qualitaetsoffensive_Lehrerbildung_Zwischenbericht_der_Evaluation_2.pdf [Zugriff: 2.5.2019].

BMBF (= Bundesministerium für Bildung und Forschung) (o.J.): Qualitätsoffensive Lehrerbildung. https://www.qualitaetsoffensive-lehrerbildung.de/de/themen-1693. html [Zugriff: 2.5.2019].

Christian-Albrechts-Universität zu Kiel (2019): Lehramt international an der CAU (LiCAU). https://www.zfl.uni-kiel.de/de/licau [Zugriff: 3.5.2019].

Criblez, Lucien (2017): Lehrerinnen- und Lehrermangel in den 1960er und frühen 1970er-Jahren - Phänomen, Maßnahmen, Wirkungen. In: Bauer, C. E./Bieri Buschor, C./Netkey, S. (Hrsg.): Berufswechsel in den Lehrberuf. Neue Wege der Professionalisierung. Bern: hep der bildungsverlag, S. 21-40.

DGfE (= Deutsche Gesellschaft für Erziehungswissenschaft) (2004a): Kerncurriculum für das Hauptfachstudium Erziehungswissenschaft. https://www.dgfe.de/fileadmin/OrdnerRedakteure/Stellungnahmen/2004_01_KC_HF_EW.pdf [Zugriff: 31.5.2019].

DGfE (= Deutsche Gesellschaft für Erziehungswissenschaft) (2004b): Strukturmodell für die Lehrerbildung im Bachelor-Master-System. https:/www.dgfe.de/fileadmin/ OrdnerRedakteure/Stellungnahmen/2004_12_Strukturmodell-BA-MA-Lehrerbildung.pdf [Zugriff: 31.5.2019].

Erdsiek-Rave, Ute (2014): Zehn Punkte. In: Erdsiek-Rave, U./John-Ohnesorg, M. (Hrsg.): Lehrerbildung im Spannungsfeld von Schulreformen und Inklusion. Schriftenreihe des Netzwerk Bildung, Bd. 30. Berlin: Friedrich-Ebert-Stiftung, S. 9-19.

Fachhochschule Südwestfalen (o.J.): Studienoption Lehramt: Vom Bachelor an der Fachhochschule an das Berufskolleg als Lehrkraft. https://www4.fh-swf.de/cms/berufsschullehrer/ [Zugriff: 15.5.2019].

GEW (= Gewerkschaft Erziehung und Wissenschaft) (2019): Vorbereitungsdienst in Teilzeit - ein Modell im Kommen. https://www.gew.de/vorbereitungsdienst/vorbereitungsdienst-in-teilzeit/ [Zugriff: 10.5.2019].

Glaser, Edith/Keiner, Edwin (2015): Unscharfe Grenzen - eine Disziplin im Dialog: Pädagogik, Erziehungswissenschaft, Bildungswissenschaft, Empirische Bildungsforschung - zur Einleitung. In: Glaser, E./Keiner, E. (Hrsg.): Unscharfe Grenzen - eine Disziplin im Dialog: Pädagogik, Erziehungswissenschaft, Bildungswissenschaft, Empirische Bildungsforschung. Bad Heilbrunn: Verlag Julius Klinkhardt, S. 7-11.

Grassmé, Isabelle/Biermann, Anje/Gläser-Zikuda, Michaela (2018): Lerngelegenheiten in Schulpraktika und ihre Bedeutung für professionsbezogene Kompetenzen von Lehramtsstudierenden. In: Rothland, M./Biederbeck, I. (Hrsg.): Praxisphasen in der Lehrerbildung im Fokus der Bildungsforschung. Münster: Waxmann, S. 15-23.

Grunert, Cathleen/Ludwig, Katja/Radhoff, Melanie/Ruberg, Christiane (2016): Studiengänge und Standorte. In: DGfE: Datenreport Erziehungswissenschaft 2016. Hrsg. v. 
Koller, H.-C./Faulstich-Wieland, H./Weishaupt, H./Züchner, I. Schriften der Deutschen Gesellschaft für Erziehungswissenschaft. Opladen: Verlag Barbara Budrich, S. 19-70. https://doi.org/10.3224/84740777A.

Häcker, Thomas/Walm, Maik (2015): Inklusion als Entwicklung - Einleitung. In: Häcker, T./Walm, M. (Hrsg.): Inklusion als Entwicklung. Konsequenzen für Schule und Lehrerbildung. Bad Heilbrunn: Klinkhardt, S. 11-26. https://doi.org/10.3224/ ezw.v26i2.21071.

HRK (= Hochschulrekorenkonferenz) (2018a): Statistische Daten zu Studienangeboten an Hochschulen in Deutschland. Studiengänge, Studierende, Absolventinnen und Absolventen. Wintersemester 2018/2019. Statistiken zur Hochschulpolitik 1/2018. https://www.hrk.de/fileadmin/redaktion/hrk/02-Dokumente/02-03-Studium/0203-01-Studium-Studienreform/HRK_Statistik_BA_MA_UEbrige_WiSe_2018_19. pdf [Zugriff: 4.11.19]

HRK (= Hochschulrekorenkonferenz) (2018b): Internationalisierung zu Hause in der Lehrerbildung. Prozessbeschreibungen aus deutschen Hochschulen. https://www. hrk.de/fileadmin/redaktion/hrk-expertise/3_Manuals/HRK-Expertise-Manual_ Lehrerbildung.pdf [Zugriff: 2.5.2019].

Horn, Klaus-Peter/Wigger, Lothar/Züchner, Ivo (2004): Neue Studiengänge - Strukturen und Inhalte. In: DGfE: Datenreport Erziehungswissenschaft 2004. Hrsg. v. Tippelt, R./Rauschenbach, T./Weishaupt, H.: Schriften der Deutschen Gesellschaft für Erziehungswissenschaft. Wiesbaden: VS Verlag für Sozialwissenschaften, S. 15-38. https://doi.org/10.1007/978-3-322-80979-7_3.

Husung, Hans-Gerhard (2014): Der Lehrerbildung mehr Gewicht verleihen. Wie Bund und Länder mit der »Qualitätsoffensive Lehrerbildung« die Ausbildung von Lehrerinnen und Lehrern an den Hochschulen aufwerten wollen. In: Hochschulrektorenkonferenz (Hrsg.): Lehrerbildung heute. Impulse für Studium und Lehre, S. 16-17. https://www.hrk.de/uploads/media/270626_HRK_Lehrerbildung_web_02.pdf [Zugriff: 20.5.2019].

Klemm, Klaus/Zorn, Dirk (2017): Demographische Rendite adé. Aktuelle Bevölkerungsentwicklung und Folgen für die allgemeinbildenden Schulen. Gütersloh: Bertelsmann-Stiftung.

Klemm, Klaus/Zorn, Dirk (2018): Lehrkräfte dringend gesucht. Bedarf und Angebot für die Primarstufe. Gütersloh: Bertelsmann-Stiftung.

KMK (= Kultusministerkonferenz) (2010): Erklärung zur Mobilität im Bildungsbereich in Europa. Beschluss der Kultusministerkonferenz vom 27.5.2010. https://www. kmk.org/fileadmin/veroeffentlichungen_beschluesse/2010/2010_05_27-Mobilitaetim-Bildungsbereich.pdf [Zugriff: 6.5.2019].

KMK (= Kultusministerkonferenz) (2017): Sachstand in der Lehrerbildung. Stand: 07.03.2017. https://www.kmk.org/fileadmin/Dateien/pdf/Bildung/AllgBildung/201703-07_Sachstand_LB_o_EW.pdf [Zugriff: 10.5.2019].

KMK (= Kultusministerkonferenz) (2018): Lehrereinstellungsbedarf und -angebot in der Bundesrepublik Deutschland 2018-2030 - Zusammengefasste Modellrechnung der Länder. Beschluss der Kultusministerkonferenz vom 11.10.2018. https://www. 
kmk.org/fileadmin/Dateien/pdf/Statistik/Dokumentationen/Dok_216_Bericht_ LEB_LEA_2018.pdf [Zugriff: 9.9.2019].

Kunter, Mareike/Kunina-Habenich, Olga/Baumert, Jürgen/Dicke, Theresa/Holzberger, Doris/Lohse-Bossenz, Hendrik/Leutner, Detlev/Schulze-Stocker, Franziska/Terhart, Ewald (2017): Bildungswissenschaftliches Wissen und professionelle Kompetenz in der Lehramtsausbildung. Ergebnisse des Projekts BilWiss. In: Gräsel, C./ Trempler, K. (Hrsg): Entwicklung von Professionalität pädagogischen Personals. Interdisziplinäre Betrachtungen, Befunde und Perspektiven. Wiesbaden: Springer VS, S. 37-54. https://doi.org/10.1007/978-3-658-07274-2. https://www.pedocs.de/ volltexte/2018/14732/pdf/LbP_2012_1_Terhart_et_al_Bildungswissenschaftliches_ Wissen_und_der_Erwerb_professioneller_Kompetenz.pdf [Zugriff: 15.8.2019].

Lindow, Ina/Shajek, Alexandra (2014): Die Professional School of Education an der Humboldt-Universität zu Berlin. In: Erdsiek-Rave, U./John-Ohnesorg, M. (Hrsg.): Lehrerbildung im Spannungsfeld von Schulreformen und Inklusion. Schriftenreihe des Netzwerk Bildung, Bd. 30. Berlin: Friedrich-Ebert-Stiftung, S. 39-44.

Mahrt, Katharina/Wehage, Heike (2014): Gemeinsam Zukunft gestalten. Aus studentischer Sicht besteht grundlegender Reformbedarf. In: Hochschulrektorenkonferenz (Hrsg.): Lehrerbildung heute. Impulse für Studium und Lehre, S. 14-15. https://www.hrk.de/uploads/media/270626_HRK_Lehrerbildung_web_02.pdf [Zugriff: 20.5.2019].

Millmann, Laura (2018): Die Zeit wird knapp im Kampf gegen den Lehrermangel. In: Grundschule 50, 8, S. 42-43.

Ministerium für Bildung, Wissenschaft und Kultur Mecklenburg-Vorpommern (2018): Merkblatt zum Vorbereitungsdienst für das Lehramt in Mecklenburg-Vorpommern zum 1. August 2018. https://www.lehrer-in-mv.de/fileadmin/Bilder_und_Dokumente_ AG/Dokumente/Dokumente_Referendare/Merkblatt_01082018.pdf [Zugriff: 11.6.2019].

Oelkers, Jürgen (2014): Rückblick nach vorn: Lehrerausbildung heute. In: Erdsiek-Rave, U./John-Ohnesorg, M. (Hrsg.): Lehrerbildung im Spannungsfeld von Schulreformen und Inklusion. Schriftenreihe des Netzwerk Bildung, Bd. 30. Berlin: FriedrichEbert-Stiftung, S. 26-35.

Oelkers, Jürgen (2018): Expertise. Struktur und Entwicklung der ersten Phase der Lehrerbildung im Freistaat Sachsen. http://www.studieren.sachsen.de/download/Expertise_Oelkers.pdf [Zugriff: 12.4.2019].

Pädagogische Hochschule Karlsruhe (2018): Bilinguales Lehren und Lernen (CLIL)/ Europalehramt. Informationen zur Bewerbung. https://www.ph-karlsruhe.de/institute/ph/chemie/bilinguales-lehren-und-lernen-clileuropalehramt/informationenzur-bewerbung [Zugriff: 3.5.2019].

Priboschek, Andrej (2016): »Heute fliegt Sie kein Pilot.«In: Grundschule 48, 9, S. 38-39. Radhoff, Melanie/Ruberg, Christiane (2018): Die Ausbildung von Sonderpädagoginnen und Sonderpädagogen im Kontext von Inklusion. In: Sonderpädagogische Förderung heute 63, 3, S. 233-244.

Radhoff, Melanie/Ruberg, Christiane/Wieckert, Sarah (2019): Die Gewissheit der Ungewissheit. Zur Professionalisierung angehender Lehrkräfte im Kontext von In- 
klusion. Sonderheft heiEDUCATION Journal »Theorie und Praxis des Lehrer/ innenhandelns«. https://heiup.uni-heidelberg.de/journals/index.php/heied/article/ view/24019 [Zugriff: 31.10.2019].

Rauschenbach, Thomas/Züchner, Ivo (2000): Studierende. In: DGfE: Datenreport Erziehungswissenschaft. Befunde und Materialien zur Lage und Entwicklung des Faches in der Bundesrepublik. Hrsg. v. Otto, H.-U./Krüger, H.-H./Merkens, H./ Rauschenbach, T./Schenk, B./Weishaupt, H./Zedler, P. Schriften der Deutschen Gesellschaft für Erziehungswissenschaft. Opladen: Leske + Budrich, S. 33-46. https:// doi.org/10.1007/978-3-322-91366-1_4.

Schubarth, Wilfried (2017): Verbesserung der Qualität des Lehramtsstudiums ist wichtiger als eine Strukturreform - Ein Kommentar. In: Schulpädagogik heute 8, 15, S. 1-5.

Stisser, Anna/Horn, Klaus-Peter/Züchner, Ivo/Ruberg, Christiane/Wigger, Lothar (2012): Studiengänge und Standorte. In: DGfE: Datenreport Erziehungswissenschaft 2012. Hrsg. v. Thole, W./Faulstich-Wieland, H./Horn, K.-P./Weishaupt, H./ Züchner, I. Schriften der Deutschen Gesellschaft für Erziehungswissenschaft. Opladen: Verlag Barbara Budrich, S. 19-69. https://doi.org/10.2307/j.ctvddzwgx.5.

Technische Hochschule Ostwestfalen-Lippe (o.J.): Jetzt den passenden Studiengang finden. Studiengänge. https://www.th-owl.de/studium/angebote/studiengaenge/?t x_in2studyfinder_pil\%5BsearchOptions\%5D\%5Btopic\%5D\%5B\%5D=51 [Zugriff: 15.5.2019].

Tenorth, Heinz-Elmar (2018): Hilfe in der Not und produktive Unruhe. https://deutsches-schulportal.de/stimmen/hilfe-in-der-not-und-produktive-unruhe/ [Zugriff: 17.5.2019].

Terhart, Ewald (Hrsg.) (2000): Perspektiven der Lehrerbildung in Deutschland. Abschlussbericht der von der Kultusministerkonferenz eingesetzten Kommission. Weinheim: Beltz.

Terhart, Ewald (2014): Dauerbaustelle Lehrerbildung. In: PÄDAGOGIK 66, 6, S. 43-47. Tillmann, Klaus-Jürgen (2012): Das Sekundarschulsystem auf dem Weg zur Zweigliedrigkeit. Historische Linien und aktuelle Verwirrungen. In: PÄDAGOGIK 64, 5, S. 8-12.

Universität Regensburg (2012): Master LINT. https://www.uni-regensburg.de/sprache-literatur-kultur/romanistik/studium/studiengaenge/master-lint/index.html [Zugriff: 3.5.2019].

Weiler, Hans Norbert (2010): Lehrerbildung und Hochschulreform - eine kritische Zwischenbilanz. In: Abel, J./Faust, G. Hrsg.): Wirkt Lehrerbildung? Antworten aus der empirischen Forschung. Münster: Waxmann, S. 15-24.

Weyand, Birgit/Krämer, Nina (2010): Erhebung von Grunddaten zu Zentren für Lehrerbildung in Deutschland. Projektbericht. Trier: Zentrum für Lehrerbildung der Universität Trier. 


\title{
3 Studienabschlüsse, Übergänge und beruflicher Verbleib der Absolventinnen und Absolventen
}

\author{
Christian Kerst \& Andrä Wolter
}

Die Erziehungswissenschaft gehört zu den "großen « Fächern an den deutschen Hochschulen, unter anderem aufgrund ihrer Doppelfunktion als eigenständiger Studiengang und als Teil der bildungswissenschaftlichen Grundausbildung in der Lehrkräfteausbildung. Dies gilt für die Studierenden- und Absolventenzahlen ebenso wie für das wissenschaftliche Personal. Damit kommt dem Fach eine besondere arbeitsmarktpolitische Bedeutung zu, die sich u.a. darin manifestiert, dass über die Berufschancen und Beschäftigungsperspektiven von Erziehungswissenschaftlerinnen und Erziehungswissenschaftlern immer wieder kontrovers diskutiert und das Fach oft als arbeitsmarktpolitisch eher prekär wahrgenommen wird. Für die Erziehungswissenschaft einschließlich der Lehrkräfteausbildung gilt wie für manch andere Fächer auch, dass die Berufschancen häufig nicht in erster Linie von Bedarfsindikatoren (wie z.B. der Nachfrage seitens der Bildungsteilnehmerinnen und -teilnehmer), sondern von politischen Entscheidungen über Ressourcenallokation abhängen, die einer anderen Logik folgen (Wolter 2015). Die Übergänge vom Studium in den Beruf und der berufliche Verbleib von Absolventinnen und Absolventen erziehungswissenschaftlicher (Hauptfach-)Studiengänge im Vergleich zu ähnlichen Studiengängen waren daher bereits in den Datenreporten 2008, 2012 und 2016 ein zentrales Kapitel. Daran anschließend werden in diesem Kapitel nach einem knappen Überblick zur Zahl der Abschlussprüfungen im Fach Erziehungswissenschaft auf der Basis der amtlichen Hochschulstatistik ${ }^{1}$ die Übergänge vom Studium in den Beruf und der längerfristige berufliche Verbleib der Absolventinnen und Absolventen mit Daten aus fächerübergreifenden Absolventenbefragungen untersucht. Eine Bilanz der bisherigen Befunde mit einer Zusammenfassung der Ergebnisse aus dem Datenreport 2020 findet sich im Abschnitt 3.4.

\subsection{Abschlussprüfungen im Spiegel der amtlichen Statistik}

Das Fach Erziehungswissenschaft wirft in der Hochschulstatistik besondere Erfassungsprobleme auf, da nicht nur affine Fächer wie Sozialpädago-

1 Wegen kurzfristiger Veränderungen im Autorenteam war es nur teilweise möglich, die in den vorangegangenen Datenreporten berichteten Statistiken zu den Abschlussprüfungen in der Erziehungswissenschaft fortzuschreiben. Deswegen fällt dieser Abschnitt hier deutlich kürzer aus. 
gik, Sozialwesen oder Sonderpädagogik, sondern auch das Unterrichtsfach Erziehungswissenschaft als Lehramtsfach, das Lehramtsstudium sowie die spezialisierten Masterstudiengänge teilweise unterschiedlich zugeordnet und codiert werden. Bezogen auf die Zahl der Erstabschlüsse, also der Absolventinnen und Absolventen, die mit einem ersten Studienabschluss die Hochschulen verlassen, liegt der Studienbereich Erziehungswissenschaften $^{2}$ auf dem 4. Platz aller 61 Studienbereiche, die in der Statistik unterschieden werden. Dieser Wert ist allerdings insofern irreführend, als in einigen Ländern (insbesondere Bayern und Baden-Württemberg) ein Teil der Lehramtsabsolventinnen und -absolventen der Primarstufe und der Sekundarstufe I hochschulstatistisch der Erziehungswissenschaft zugerechnet wird. Um einen Eindruck von der relativen Größe des Faches zu bekommen, ist es deshalb sinnvoller, nur die Bachelorabschlüsse im Erststudium zu betrachten. Hier erreicht die Erziehungswissenschaft den 8. Rang. Dabei gibt es bekanntlich große Unterschiede nach dem Geschlecht. Bei den Männern liegt die Erziehungswissenschaft (BA Erstabschluss) auf Rang 29, bei den Frauen auf Rang 3, hinter den Studienbereichen Wirtschaftswissenschaften und Sozialwesen. Bei den Masterabschlüssen und den Promotionen rangiert das Fach ebenfalls unter den 20 am stärksten besetzten Studienbereichen, allerdings auf den Rangplätzen 11 beim Master und 16 bei der Promotion (Tabelle 3.1).

Die Zahl der Bachelorabschlüsse an Universitäten ist in der Erziehungswissenschaft mit zunehmender Umstellung auf die gestufte Studienstruktur gestiegen, zuletzt auf ca. 6.000 pro Jahr (Tabelle 3.2). Die Zahl der Erstabschlüsse (Bachelor, traditionelle Abschlüsse) liegt in den letzten Jahren höher als je zuvor. Die Attraktivität des Faches scheint also eher zuzunehmen, auch wenn die Zahl der weiterführenden Abschlüsse rückläufig ist. Außerdem gewinnt der Studienbereich Erziehungswissenschaft in der Hochschulstatistik an den Fachhochschulen mit weiteren 1.500 Abschlüssen an Bedeutung ${ }^{3}$, wobei etwa zwei Drittel in einem Studiengang abschließen, der statistisch der Erziehungswissenschaft zugerechnet wird, ein weiteres Drittel im Fach Pädagogik der frühen Kindheit. Weiter ausgebaut wurde in den letzten Jahren auch das Sozialwesen an Fachhochschulen. Die Zahl der Lehramtsabschlüsse (Staatsexamen und Master) schwankt seit 2011 um 30.000.

2 Die 61 Studienbereiche der Hochschulstatistik umfassen i.d.R. jeweils mehrere Studienfächer. Zum Studienbereich Erziehungswissenschaften gehören neben dem größten Fach Erziehungswissenschaft, auf das $201780 \%$ aller Bachelorabschlüsse entfallen, weitere kleinere Fächer wie z.B. Sonderpädagogik (6 \%), Erwachsenenbildung (2 \%) oder Pädagogik der frühen Kindheit (7\%).

3 Ohne Tabelle. Man erkennt die Größenordnung, wenn man die Zahl der Bachelorabschlüsse in der Erziehungswissenschaft in den Tabellen 3.1 und 3.2 vergleicht. 
Tabelle 3.1: Bestandene Abschlussprüfungen in den am stärksten besetzten Studienbereichen $2017^{1}$

\begin{tabular}{|c|c|c|c|c|c|c|}
\hline Rang & Studienbereich & $\begin{array}{c}\mathrm{BA}^{2} \\
\text { Anzahl }\end{array}$ & Studienbereich & $\begin{array}{c}\mathrm{MA}^{3} \\
\text { Anzahl }\end{array}$ & Studienbereich & $\begin{array}{l}\text { Prom } \\
\text { Anzahl }\end{array}$ \\
\hline 1 & $\begin{array}{l}\text { Wirtschaftswissen- } \\
\text { schaften }\end{array}$ & 58.185 & $\begin{array}{l}\text { Wirtschaftswissen- } \\
\text { schaften }\end{array}$ & 27.880 & Humanmedizin & 6.090 \\
\hline 2 & $\begin{array}{l}\text { Maschinenbau, } \\
\text { Verfahrenstechnik }\end{array}$ & 20.375 & $\begin{array}{l}\text { Maschinenbau, } \\
\text { Verfahrenstechnik }\end{array}$ & 12.610 & Biologie & 2.715 \\
\hline 3 & Informatik & 15.580 & Informatik & 8.805 & Chemie & 2.275 \\
\hline 4 & Sozialwesen & 13.235 & $\begin{array}{l}\text { Elektrotechnik und } \\
\text { Informationstechnik }\end{array}$ & 6.175 & $\begin{array}{l}\text { Maschinenbau, } \\
\text { Verfahrenstechnik }\end{array}$ & 1.835 \\
\hline \multirow[t]{2}{*}{5} & $\begin{array}{l}\text { Gesundheitswissen- } \\
\text { schaften allgemein }\end{array}$ & 8.025 & Psychologie & 4.780 & Physik, Astronomie & 1.795 \\
\hline & $\ldots$ & & $\ldots$ & & $\ldots$ & \\
\hline \multirow[t]{2}{*}{8} & $\begin{array}{l}\text { Erziehungswissen- } \\
\text { schaften }\end{array}$ & 7.400 & Bauingenieurwesen & 4.055 & Informatik & 975 \\
\hline & $\cdots$ & & $\cdots$ & & $\cdots$ & \\
\hline \multirow[t]{2}{*}{11} & Bauingenieurwesen & 5.710 & $\begin{array}{l}\text { Erziehungswissen- } \\
\text { schaften }\end{array}$ & 3.355 & Mathematik & 655 \\
\hline & $\ldots$ & & $\cdots$ & & $\cdots$ & \\
\hline 16 & $\begin{array}{l}\text { Wirtschaftsingenieur- } \\
\text { wesen mit wirtschafts- } \\
\text { wiss. Schwerpunkt }\end{array}$ & 4.355 & $\begin{array}{l}\text { Agrarwissenschaften, } \\
\text { Lebensmittel- und } \\
\text { Getränketechnologie }\end{array}$ & 2.330 & $\begin{array}{l}\text { Erziehungswissen- } \\
\text { schaften }\end{array}$ & 410 \\
\hline
\end{tabular}

1 an Universitäten (einschließlich der Pädagogischen, Theologischen und Kunsthochschulen) und Fachhochschulen

2 Abschluss eines Erststudiums

3 Abschluss eines Folgestudiums

Quelle: Statistisches Bundesamt, Hochschulstatistik, Recherche in DZHW-ICE (Werte auf ein Vielfaches von 5 gerundet)

Seit der Umstellung auf die gestuften Abschlüsse ist die Zahl der traditionellen Abschlüsse Diplom und Magister stark zurückgegangen, auch wenn sie vereinzelt immer noch vorkommen (Tabelle 3.2). Parallel dazu ist die Zahl der Masterabschlüsse stark gestiegen. Insgesamt ist allerdings die Zahl der Erziehungswissenschaftlerinnen und Erziehungswissenschaftler gesunken, die einen Abschluss auf dem Niveau der traditionellen Abschlüsse (Diplom, Magister, Master) erwerben, von 4.870 im Jahr 2010 auf 3.185 im Jahr 2018. Dieser Rückgang ist vor allem auf die niedrige und tendenziell rückläufige Übergangsquote vom Bachelor- zum Masterstudium zurückzuführen, die in der Erziehungswissenschaft deutlich geringer ausfällt als in den Vergleichs- 
fächern (siehe dazu Abschnitt 3.2.2). ${ }^{4}$ Offensichtlich entscheiden sich viele Absolventinnen und Absolventen, nach dem Bachelor direkt in eine Erwerbstätigkeit überzugehen. Da mehr als die Hälfte aller Masterstudiengänge in der Erziehungswissenschaft nicht zulassungsbeschränkt ist und die Zulassungsquoten sehr hoch ausfallen, können Engpässe im Angebot dabei nur eine begrenzte Rolle spielen.

Im Fächervergleich zeigt sich, dass auch in den Sozial- und Politikwissenschaften die Zahl der Abschlüsse auf dem Niveau von Diplom, Magister und Master zurückgeht, allerdings etwas weniger stark als in der Erziehungswissenschaft, wo 2018 nur noch $65 \%$ der Abschlüsse von 2010 erreicht werden (Sozial-/Politikwissenschaft: 70 \%). In der stark nachgefragten Psychologie ist die Zahl der Abschlüsse auf dem Masterniveau hingegen deutlich gestiegen und liegt 2018 um 31 \% über dem Wert von 2010. In den Wirtschaftswissenschaften ist die Zahl der Abschlüsse auf diesem Niveau nach einem Rückgang bis 2012 wieder angestiegen und erreicht 2018 den Wert von 2010.

Tabelle 3.2: Bestandene Prüfungen nach Abschlussart ${ }^{1}$ im Fächervergleich ${ }^{2}$ (1995-2018)

\begin{tabular}{|c|c|c|c|c|c|c|c|c|c|c|c|c|}
\hline & & ziehung & viss. (Un & & & Psychol & gie (Uni) & & & ial-/Poli & kwiss. (L & \\
\hline & & & & trad. \& & & & & trad. \& & & & & trad. \& \\
\hline & trad. & BA & MA & MA & trad. & BA & $M A$ & MA & trad. & BA & MA & MA \\
\hline 1995 & 1.905 & 0 & 0 & 1.905 & 2.330 & 0 & 0 & 2.330 & 2.095 & 0 & 0 & 2.095 \\
\hline 2000 & 3.555 & 15 & 0 & 3.555 & 2.595 & 10 & 0 & 2.595 & 2.675 & 5 & 0 & 2.675 \\
\hline 2005 & 3.900 & 170 & 650 & 4.550 & 3.310 & 135 & 20 & 3.330 & 4.160 & 415 & 100 & 4.260 \\
\hline 2010 & 4.240 & 2.235 & 630 & 4.870 & 3.120 & 1.490 & 170 & 3.290 & 4.915 & 3.255 & 820 & 5.735 \\
\hline 2011 & 3.380 & 2.935 & 990 & 4.370 & 3.050 & 2.255 & 270 & 3.320 & 4.000 & 4.255 & 1.400 & 5.400 \\
\hline 2012 & 2.770 & 3.905 & 1.305 & 4.075 & 2.640 & 2.625 & 625 & 3.265 & 3.080 & 5.060 & 1.825 & 4.905 \\
\hline 2013 & 1.895 & 4.390 & 1.910 & 3.805 & 2.195 & 3.490 & 1.400 & 3.595 & 1.865 & 5.595 & 2.215 & 4.080 \\
\hline 2014 & 1.175 & 4.985 & 2.315 & 3.490 & 1.405 & 4.120 & 2.200 & 3.605 & 1.340 & 5.830 & 2.910 & 4.250 \\
\hline 2015 & 660 & 5.300 & 2.695 & 3.355 & 705 & 4.285 & 3.000 & 3.705 & 680 & 6.100 & 3.260 & 3.940 \\
\hline 2016 & 445 & 5.945 & 3.175 & 3.620 & 435 & 4.465 & 3.485 & 3.920 & 405 & 6.050 & 3.405 & 3.810 \\
\hline 2017 & 305 & 5.875 & 3.290 & 3.595 & 310 & 4.675 & 3.740 & 4.050 & 210 & 6.565 & 3.820 & 4.030 \\
\hline 2018 & 60 & 6.005 & 3.125 & 3.185 & 165 & 4.700 & 4.150 & 4.315 & 130 & 6.085 & 3.910 & 4.040 \\
\hline
\end{tabular}

4 Setzt man die Zahl der Masterabschlüsse mit den Bachelorabschlüssen zwei Jahre zuvor ins Verhältnis, ergibt sich für die Erziehungswissenschaft ein Wert von 53 \%, in der Psychologie von $93 \%$, in den Sozial- und Politikwissenschaften von $85 \%$, in den universitären Wirtschaftswissenschaften von ca. $79 \%$. Diese überschlägig berechneten Masterquoten korrespondieren (mit Ausnahme der Wirtschaftswissenschaften) in der Tendenz mit den Masterquoten, die sich mit dem in Tabelle 3.3 verwendeten Verfahren ergeben. 
Studienabschlüsse und Verbleib

\begin{tabular}{|c|c|c|c|c|c|c|c|c|c|c|}
\hline & \multicolumn{4}{|c|}{ Wirtschaftswiss. (Uni) } & \multicolumn{3}{|c|}{ Lehramt $^{4}$} & \multicolumn{3}{|c|}{ Sozialwesen (FH) } \\
\hline & trad. & BA & MA & $\begin{array}{c}\text { trad. \& } \\
\text { MA }\end{array}$ & $\begin{array}{l}\text { Staats- } \\
\text { examen }\end{array}$ & $\begin{array}{c}\text { Master } \\
\text { LA }\end{array}$ & $\begin{array}{c}\text { Lehramt } \\
\text { insgesamt }\end{array}$ & trad. & BA & MA \\
\hline 1995 & 16.950 & 0 & 0 & 16.950 & 22.525 & & 22.525 & 7.620 & 0 & 0 \\
\hline 2000 & 12.555 & 5 & 0 & 12.555 & 22.625 & & 22.625 & 8.115 & 0 & 0 \\
\hline 2005 & 14.370 & 930 & 1.415 & 15.785 & 21.250 & 0 & 21.250 & 8.015 & 0 & 80 \\
\hline 2010 & 13.510 & 10.105 & 2.620 & 16.130 & 26.945 & 4.295 & 31.240 & 5.195 & 6.090 & 450 \\
\hline 2011 & 10.420 & 13.870 & 4.195 & 14.615 & 25.030 & 5.600 & 30.630 & 2.435 & 8.095 & 720 \\
\hline 2012 & 6.490 & 16.050 & 7.455 & 13.945 & 21.480 & 7.905 & 29.385 & 1.505 & 8.755 & 1.030 \\
\hline 2013 & 3.515 & 17.330 & 10.800 & 14.315 & 20.580 & 9.825 & 30.405 & 515 & 10.530 & 1.205 \\
\hline 2014 & 1.705 & 19.555 & 12.740 & 14.445 & 19.435 & 10.800 & 30.235 & 185 & 10.670 & 1.370 \\
\hline 2015 & 675 & 20.335 & 14.435 & 15.110 & 18.165 & 11.315 & 29.480 & 60 & 11.145 & 1.515 \\
\hline 2016 & 385 & 20.215 & 15.240 & 15.625 & 17.325 & 12.265 & 29.590 & 20 & 11.530 & 1.655 \\
\hline 2017 & 185 & 19.905 & 15.990 & 16.175 & 16.290 & 13.925 & 30.215 & 5 & 11.830 & 1.725 \\
\hline 2018 & 170 & 19.575 & 15.930 & 16.100 & - & - & - & 0 & 12.095 & 1.865 \\
\hline
\end{tabular}

1 trad. = Diplom und Magister, $\mathrm{BA}=$ Bachelor (ohne Bachelor LA), $\mathrm{MA}=$ Master (ohne Master LA). Die traditionellen Abschlüsse sowie die Bachelorabschlüsse sind Abschlüsse nach einem Erststudium; die Masterabschlüsse sind Abschlüsse eines Folgestudiums.

2 Basis sind die Studienbereiche der Hochschulstatistik.

3 Erziehungswissenschaft ohne Sonderpädagogik und Sozialpädagogik

4 Werte für 2018 sind noch nicht verfügbar.

Quelle: Statistisches Bundesamt, Hochschulstatistik, Recherche in DZHW-ICE (Werte auf ein Vielfaches von 5 gerundet)

\section{2 Übergänge und Verbleib der Absolventinnen und Absolventen}

\subsubsection{Fragestellungen und Datenbasis}

Die Analysen aus den früheren Datenreporten zu den Übergängen und zum Verbleib von Erziehungswissenschaftlerinnen und Erziehungswissenschaftlern im Vergleich zu den anderen hier betrachteten Studienfächern werden in diesem Abschnitt mit neuen Datensätzen und einem erweiterten Beobachtungszeitraum fortgeschrieben. Dazu werden wieder Daten des DZHW(früher HIS-)Absolventenpanels genutzt. Erstmals können nun auf der Basis von Daten, die zur letzten Ausgabe noch nicht vorlagen, die Übergänge nach dem Bachelor- und Masterabschluss betrachtet werden. Im Zentrum stehen dabei die Tätigkeiten, die nach diesen Abschlüssen ausgeübt werden, insbe- 
sondere die Übergangsquote in den Master sowie die Situation kurz nach dem Examen. Abschließend soll die Berichterstattung zu den Merkmalen der Erwerbstätigkeit in mittel- und langfristiger Perspektive fortgeschrieben werden. Während in den früheren Absolventenkohorten immer noch ein hoher Anteil der »alten « Abschlüsse (Diplom, Magister) vertreten war, sind in den jüngeren Kohorten nunmehr steigende Absolventenzahlen mit Bachelor oder Master zu finden. Für Analysen zum langfristigen Verbleib der Absolventinnen und Absolventen spielen die "alten « Abschlüsse aber immer noch eine wichtige Rolle, da langfristige Verbleibsdaten für die Bachelor- und Masterabschlüsse (z.B. um längerfristige monetäre und nicht-monetäre Bildungserträge ermitteln zu können) noch gar nicht vorliegen. Eine Rolle spielt auch, dass aufgrund der relativ hohen Übergangsquoten in ein Masterstudium in vielen Fächern nur ein kleinerer Teil der Absolventinnen und Absolventen nach dem Bachelorabschluss erwerbstätig wird.

Wichtigste Datenbasis sind jeweils die in einem Kohorten-Längsschnittdesign durchgeführten DZHW-Absolventenstudien, die mehrere Vorteile bieten: Sie ermöglichen sowohl eine Längsschnittperspektive wie eine Zeitreihe und umfassen eine repräsentative Auswahl. Für das DZHW-Absolventenpanel wird eine bundesweit repräsentativ gezogene Stichprobe von Absolventinnen und Absolventen aller Studienfächer - seit 1989 jeder vierte Prüfungsjahrgang - zu drei Zeitpunkten nach dem Studienabschluss befragt. Die erste Befragung findet ca. ein Jahr nach dem Studienabschluss statt, eine zweite Welle ca. fünf Jahre nach dem Hochschulabschluss. Seit dem Prüfungsjahrgang 1997 werden die Kohorten zehn Jahre nach dem Abschluss zum dritten Mal befragt. Zur Verfügung stehen für diesen Datenreport die Surveys der Prüfungsjahrgänge 1997, 2001 und 2005 mit jeweils drei Wellen, der Prüfungsjahrgang 2009 mit zwei Wellen und der Prüfungsjahrgang 2013 mit einer Welle. ${ }^{5}$ In diesen Datensätzen gibt es, mit Ausnahme der Kohorten 2009 und 2013, ganz überwiegend Befragte mit Diplom- oder Magisterabschlüssen. Für die Auswertungen zum Verbleib der Bachelor- und Masterabsolventinnen und -absolventen in der ersten Zeit nach dem Studienabschluss wird auf die ersten Wellen der Abschlussjahrgänge 2009 und 2013 zurückgegriffen. Aktuellere Daten des Jahrgangs 2017 liegen bis zum Redaktionsschluss für diese Ausgabe des Datenreports leider noch nicht vor. Analysen nach dem Geschlecht sind mit den Daten nicht möglich, da angesichts eines Frauenanteils von $90 \%$ und mehr kaum männliche Absolventen in der Erziehungswissenschaft vorhanden sind.

5 Die Zahl der Absolventinnen und Absolventen aus der Erziehungswissenschaft, die sich an den bis zu drei Wellen der Absolventenkohorten 2009 und 2013 beteiligt haben, ist unterschiedlich. Die den Analysen zugrundliegenden Fallzahlen sind in den Tabellen angegeben. 


\subsubsection{Verbleib nach dem Bachelorabschluss}

\section{Übergang in das Masterstudium}

Mit dem Bachelorabschluss stehen Hochschulabsolventinnen und -absolventen vor der Entscheidung, eine Erwerbstätigkeit oder ein Masterstudium aufzunehmen. Bisher fällt diese Entscheidung, zumindest bei Studierenden mit einem universitären Bachelorabschluss, überwiegend zugunsten eines Masterstudiums aus (vgl. Autorengruppe Bildungsberichterstattung 2018, Tabelle F57web, Autorengruppe Bildungsberichterstattung 2016, Tabelle F4-10web). Die Übergangsquoten über alle Fächer haben sich, je nach verwendeter Datenquelle, bei etwa 70 bis $80 \%$ stabilisiert. Während der Masterabschluss weitgehend dem früheren Diplom oder Magister entspricht, wird offensichtlich der Bachelor im Universitätsbereich kaum als berufsqualifizierender Abschluss akzeptiert. Dagegen liegt die Übergangsquote in den Master mit dem Bachelorabschluss einer Fachhochschule niedriger. Der Bachelorabschluss ist in seiner Verwertbarkeit am Arbeitsmarkt offenkundig weitgehend dem früheren Fachhochschuldiplom vergleichbar (vgl. Autorengruppe Bildungsberichterstattung 2018: 169). Mit 30 bis 40 \% schließen dennoch deutlich mehr Fachhochschulabsolventinnen und -absolventen ein weiteres Studium an, als es früher beim Fachhochschuldiplom der Fall war. Ursprüngliche Erwartungen, dass der Bachelorabschluss zum Regelabschluss wird und nur ein kleinerer Teil in das Masterstudium übergeht, haben sich somit bisher nicht realisiert. Die Masterquote unterscheidet sich jedoch nicht nur nach der Art der besuchten Hochschule, sondern auch nach Fachrichtungen. Für einige Fachrichtungen ist ein Master formal (Lehramt) oder faktisch (z.B. Naturwissenschaften) erforderlich, um den angestrebten Beruf ausüben zu können.

Um die Masterquote für die Erziehungswissenschaft und die Vergleichsfächer zu ermitteln, stehen zwei Datenquellen zur Verfügung: die amtliche Hochschulstatistik und Absolventenbefragungen. Mit der Hochschulstatistik ist eine Abschätzung der Masterquoten möglich, indem die Zahl der Bachelorabschlüsse eines Prüfungsjahres mit den Masterstudierenden im 1. Studienjahr des Folgejahres ins Verhältnis gesetzt wird. Eine durch verschiedene Surveys gestützte Annahme besteht dabei darin, dass das Masterstudium in den meisten Fällen unmittelbar nach dem Bachelorabschluss aufgenommen wird. Masterübergänge, die erst nach einer Phase der Erwerbstätigkeit erfolgen, können mit diesem Verfahren nicht berücksichtigt werden. ${ }^{6}$ Außerdem

6 Werte über $100 \%$ kommen zustande, wenn Masterstudierende aus früheren Jahren in die Quote des aktuellen Jahres eingerechnet werden. Auch Masterstudierende, die vorher keinen Bachelor erworben haben, sondern etwa ein Diplom, und zu einem späteren Zeitpunkt noch ein Masterstudium aufnehmen, können zur Überschätzung der Masterquote beitragen. Umgekehrt wird die Masterquote bei diesem Verfahren unterschätzt, wenn ein größerer Teil der Absolventinnen und Absolventen eines Fachs nicht im ersten Jahr nach dem Abschluss mit dem Masterstudium beginnt. 
werden Wechsel zwischen den Hochschularten sowie Fachwechsel zwischen Bachelor und Master, etwa von den Gesellschafts- in die Erziehungswissenschaften oder umgekehrt, nicht berücksichtigt. So müssen die Anfängerinnen und Anfänger in den Masterstudiengängen der Erziehungswissenschaft nicht notwendig einen erziehungswissenschaftlichen Bachelorabschluss besitzen. Trotz dieser Einschränkungen vermittelt das Verfahren einen Eindruck von der Größenordnung der Masterquote und den Unterschieden zwischen den Fachrichtungen (Tabelle 3.3). ${ }^{7}$

Tabelle 3.3: Studierende ${ }^{1}$ im ersten Studienjahr des Masterstudiums ${ }^{2}$ bezogen auf die Anzahl der Bachelorabschlüsse 2010 bis $2017^{3}$

\begin{tabular}{ccccccccc} 
Jahr & $\begin{array}{l}\text { Erzie- } \\
\text { hungs- } \\
\text { wiss. BA }\end{array}$ & $\begin{array}{l}\text { Psycho- } \\
\text { logie BA }\end{array}$ & $\begin{array}{l}\text { Sozial-, } \\
\text { Politik- } \\
\text { wiss. BA }\end{array}$ & $\begin{array}{l}\text { Wirt- } \\
\text { schafts- } \\
\text { wiss. BA }\end{array}$ & $\begin{array}{l}\text { Sozial- } \\
\text { wesen BA } \\
\text { (FH) }\end{array}$ & $\begin{array}{l}\text { Hoch- } \\
\text { schulen } \\
\text { insgesamt }\end{array}$ & $\begin{array}{l}\text { Uni- } \\
\text { versitäten }\end{array}$ & $\begin{array}{l}\text { Fachhoch- } \\
\text { schulen }\end{array}$ \\
2010 & 73,2 & 98,0 & 102,7 & 67,5 & 26,7 & 71,8 & 102,1 & 43,7 \\
\hline 2011 & 78,4 & 92,7 & 91,0 & 60,1 & 24,4 & 65,6 & 96,6 & 37,7 \\
\hline 2012 & 61,8 & 114,1 & 86,5 & 57,9 & 23,2 & 64,1 & 95,4 & 35,9 \\
\hline 2013 & 62,2 & 111,1 & 86,6 & 60,0 & 21,7 & 65,4 & 97,3 & 36,8 \\
\hline 2014 & 56,8 & 108,3 & 86,5 & 56,8 & 23,7 & 64,3 & 93,9 & 37,1 \\
\hline 2015 & 50,6 & 112,4 & 80,0 & 54,3 & 22,3 & 62,5 & 91,2 & 36,9 \\
\hline 2016 & 46,7 & 110,6 & 78,7 & 53,7 & 24,6 & 62,0 & 91,1 & 37,7 \\
\hline 2017 & 49,0 & 102,4 & 75,4 & 54,2 & 23,5 & 61,3 & 89,7 & 38,2
\end{tabular}

1 Nur Deutsche und Bildungsinländerinnen/Bildungsinländer

2 Studierende, die im jeweiligen Wintersemester im 1. oder 2. Fachsemester des Masterstudiums eingeschrieben sind

3 Die Lehramtsstudiengänge können hier nicht ausgewiesen werden, weil die Bezugsgröße für die Studierenden in Lehramts-Masterstudiengängen nicht bekannt ist; denn nur ein kleiner Teil der Studierenden im Lehramts-Masterstudium hat zuvor den Abschluss Bachelor (Lehramt) erworben.

4 Einschließlich Verwaltungsfachhochschulen

Quelle: Statistisches Bundesamt, Hochschulstatistik, Recherche in DZHW-ICE, eigene Berechnungen

Unter den Vergleichsfächern ist der große Unterschied zwischen den Bachelorabsolventinnen und -absolventen aus Universitäten und Fachhochschulen deutlich erkennbar (Tabelle 3.3). An den Universitäten liegt die Masterquote, einschließlich der Masterstudierenden, die von den Fachhochschulen komanalysiert werden. Dann kann auch der zeitliche Abstand zwischen Bachelorabschluss und Masterstudium ebenso berücksichtigt werden wie Fachwechsel zwischen beiden Studienphasen. 
men, seit 2014 bei etwa $90 \%$, an den Fachhochschulen bei etwas unter $40 \%$ (Tabelle 3.3). Die Unterschiede zwischen den Fachrichtungen sind ebenfalls beträchtlich. Als vorwiegend an Fachhochschulen angebotenes Fach ist die Masterquote im Sozialwesen mit knapp einem Viertel am geringsten. Abgesehen vom Sozialwesen liegt die Quote in der Erziehungswissenschaft mit zuletzt unter $50 \%$ am niedrigsten und deutlich unter dem Wert für die Universitäten insgesamt. Die Wirtschaftswissenschaften zeigen mit etwas mehr als $50 \%$ ebenfalls einen unterdurchschnittlichen Anteil, während in den Sozial- und Politikwissenschaften mit drei Viertel und der Psychologie mit annähernd $100 \%$ die meisten Bachelors ein Masterstudium aufnehmen. Im Zeitverlauf ist in den meisten der ausgewiesenen Fächer eine leichte Abnahme der Masterquoten zu erkennen, wobei die deutlich höheren Quoten zu Beginn des Jahrzehnts nicht überbewertet werden sollten. In dieser Zeit kam ein Teil der Masterstudierenden aus Diplom- oder Magisterstudiengängen, die inzwischen weitgehend ausgelaufen sind.

In Absolventenstudien wird erfragt, ob Bachelors ein Masterstudium - im Fach des Bachelorabschlusses oder in einem anderen Fach - aufgenommen haben oder dies für die Zukunft planen. Dabei werden auch verzögerte Übergänge in das Masterstudium erfasst. In der Größenordnung und den Unterschieden zwischen den Fächern sind die ermittelten Werte für die Absolventenjahrgänge 2013 und 2009 tendenziell mit denen vergleichbar, die mit dem hochschulstatistischen Verfahren ermittelt wurden. Auch hier zeigen sich deutliche Fächerunterschiede (Tabelle 3.4). Im Lehramt sowie in der Psychologie sind mit über $90 \%$ die höchsten Masterquoten zu erkennen. Da für den Lehrerberuf der Abschluss eines Masterstudiums erforderlich ist, bildet der Masterabschluss hier quasi den Regelabschluss. Ähnlich ist es in der Psychologie, wo die fachspezifischen Weiterbildungen den Master voraussetzen. In den übrigen drei universitären Vergleichsfächern erreicht die Masterquote in der Erziehungswissenschaft mit unter $70 \%$ den geringsten Wert. Die Masterquote in den Wirtschaftswissenschaften liegt etwas über der in der Erziehungswissenschaft, während die Quote in den Sozial- und Politikwissenschaften wie in der hochschulstatistischen Analyse ein höheres Niveau erreicht. Im Sozialwesen (Abschluss an einer Fachhochschule) ${ }^{8}$ liegt die Masterquote mit $30 \%$ am niedrigsten; sie ist damit auch niedriger als im Durchschnitt der Fachhochschulen (ca. 50 \% in den Absolventenjahrgängen 2013 und 2009). Allerdings gibt ein Teil derjenigen, die nach dem ersten Abschluss kein Masterstudium aufgenommen haben, an, noch zu einem späteren Zeitpunkt ein Masterstudium aufnehmen zu wollen (»Master geplant«). In der Erziehungswissenschaft sind das $15 \%$ (Tabelle 3.4). 
Tabelle 3.4: Übergang von Bachelorabsolventinnen und -absolventen in ein Masterstudium 2013 und 2009 ${ }^{1}$, Zahl der Bewerbungen und Zusagen und Hochschule des Masterstudiums 2013 (in \%)

\begin{tabular}{|c|c|c|c|c|c|c|c|c|}
\hline & 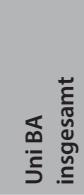 & 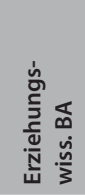 & 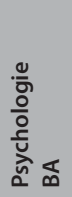 & 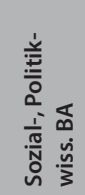 & 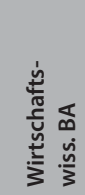 & 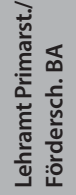 & 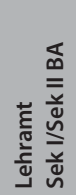 & 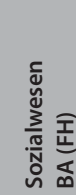 \\
\hline & \multicolumn{8}{|c|}{ (in \%) } \\
\hline $\begin{array}{l}\text { Masterstudium } \\
\text { aufgenommen }{ }^{1}\end{array}$ & $82[72]$ & $69[66]$ & $91[/]$ & $78[78]$ & $73[72]$ & $96[-]$ & $96[-]$ & $29[30]$ \\
\hline $\begin{array}{l}\text { darunter } \\
\text { Masterstudium in Teilzeit }{ }^{2}\end{array}$ & $3[-]$ & $16[-]$ & $2[-]$ & $3[-]$ & $5[-]$ & $3[-]$ & $0[-]$ & $47[-]$ \\
\hline $\begin{array}{l}\text { Masterstudium } \\
\text { später geplant }\end{array}$ & 8 & 15 & 7 & 13 & 14 & 2 & 3 & 27 \\
\hline \multicolumn{9}{|l|}{$\begin{array}{l}\text { Bewerbungen auf das } \\
\text { Masterstudium } 2013\end{array}$} \\
\hline$\ldots$ an der BA-Hochschule & 86 & 75 & 90 & 68 & 84 & 92 & 100 & 60 \\
\hline ... an anderer Hochschule & 58 & 78 & 83 & 84 & 73 & 30 & 36 & 57 \\
\hline \multicolumn{9}{|l|}{$\begin{array}{l}\text { Zusagen für ein } \\
\text { Masterstudium } 2013\end{array}$} \\
\hline$\ldots$ an der BA-Hochschule & 96 & 96 & 84 & 90 & 89 & 99 & 100 & 90 \\
\hline .... an anderer Hochschule & 92 & 94 & 88 & 93 & 92 & 92 & 96 & 93 \\
\hline \multicolumn{9}{|l|}{$\begin{array}{l}\text { Hochschule des } \\
\text { Masterstudiums } 2013\end{array}$} \\
\hline $\begin{array}{l}\text {... Hochschule des BA- } \\
\text { Abschlusses }\end{array}$ & 73 & 51 & 56 & 44 & 60 & 94 & 94 & 48 \\
\hline $\begin{array}{l}\ldots \text { andere Hochschule im } \\
\text { Inland }\end{array}$ & 24 & 48 & 42 & 48 & 33 & 5 & 6 & 49 \\
\hline $\begin{array}{l}\text {... Hochschule im } \\
\text { Ausland }\end{array}$ & 3 & 0 & 2 & 8 & 7 & 0 & 0 & 4 \\
\hline$n$ & $\begin{array}{r}7.325 \\
{[2.595]}\end{array}$ & $\begin{array}{r}396 \\
{[150]}\end{array}$ & $\begin{array}{l}285 \\
{[77]}\end{array}$ & $\begin{array}{r}499 \\
{[236]}\end{array}$ & $\begin{array}{r}897 \\
{[468]}\end{array}$ & $\begin{array}{r}289 \\
{[-]}\end{array}$ & $\begin{array}{r}191 \\
{[-]}\end{array}$ & $\begin{array}{r}469 \\
{[356]}\end{array}$ \\
\hline
\end{tabular}

1 Vergleichswert für Kohorte 2009 [in Klammern]

2 Nur Befragte mit Bachelorabschluss aus der DZHW-Teilstichprobe des Absolventenpanels 2013.1

Quelle: DZHW-Absolventenpanel 2013.1 und 2009.1 (- = keine Daten vorhanden; / = Wert nicht belastbar, kleine Fallzahl)

Berufsbegleitende Masterstudiengänge sind an Universitäten selten; allenfalls Masterstudierende an Fachhochschulen sind zu einem größeren Anteil in berufsbegleitende Teilzeit-Masterstudiengänge eingeschrieben (über alle Fächer $24 \%$, nicht in Tabelle 3.4). Eine nennenswerte Rolle spielen sie in der Erziehungswissenschaft (16\%) und im Sozialwesen (47 \%). 
Im Vergleich der beiden verfügbaren Absolventenbefragungen 2009 und 2013 ist keine Abnahme der Masterquote zu erkennen; bei den universitären Bachelors steigt sie sogar leicht an. ${ }^{9}$ Der Masterabschluss scheint sich als Regelabschluss nach einem universitären Bachelorstudium zu etablieren. Dazu trägt vermutlich auch bei, dass keine größeren Hürden beim Zugang zum Masterstudium zu erkennen sind (s. dazu auch Abbildung 3.1).

Abbildung 3.1: Gründe gegen die Aufnahme eines Masterstudiums nach Fachrichtungen (in \%)

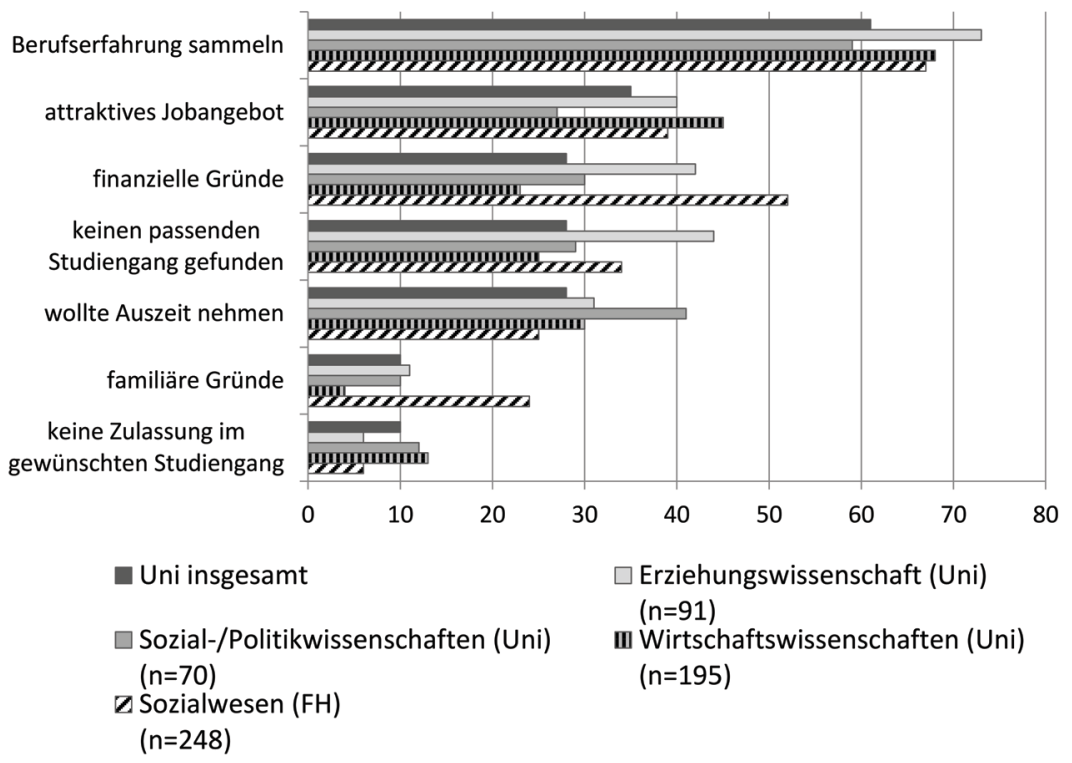

Quelle: DZHW-Absolventenpanel 2013.1

Die meisten Absolventinnen und Absolventen, die ein Masterstudium anstreben, bewerben sich nach dem Bachelorstudium sowohl an der Hochschule ihres Bachelorabschlusses als auch an einer anderen Hochschule (Tabelle 3.4). Die Zulassungsquoten sind sowohl an der Heimathochschule als auch an den anderen Hochschulen sehr hoch und liegen über $90 \%$, bei Bewerbungen auf LA-Master sogar bei $100 \%$. Zumindest in diesen Absolventenkohorten scheint es nur sehr selten vorgekommen zu sein, überhaupt keinen Masterstudienplatz zu erhalten. Insgesamt sind etwa drei Viertel der Masterstudierenden des Absol-

9 In der Absolventenbefragung 2013 kann aufgrund aus einer anderen Erhebung zugespielter Daten bei weiterführenden Studiengängen nicht nach Master und anderen Abschlüssen unterschieden werden. Deshalb erfolgt eine geringe Überschätzung der Masterquote, die auf maximal 3 bis $5 \%$ geschätzt wird (vgl. Fabian/Hillmann/Trennt/Briedis 2016: 57). 
ventenjahrgangs 2013 an der Hochschule ihres Bachelorabschlusses geblieben (Tabelle 3.4). Dieser Wert wird jedoch durch die sehr hohen Verbleibsquoten in den LA-Studiengängen beeinflusst. Bachelors der Erziehungswissenschaft verbleiben nur zu etwa $50 \%$ an der Hochschule des Bachelorabschlusses. Noch geringer ist dieser Anteil nur in den Sozial- und Politikwissenschaften, wo auch der Auslandsanteil mit $8 \%$ am höchsten ist. Ein Wechsel zum Masterstudium ins Ausland spielt dagegen in der Erziehungswissenschaft keine Rolle.

Der wichtigste Grund, (zunächst) kein Masterstudium aufzunehmen, liegt darin, erst einmal Berufserfahrung sammeln zu wollen. In der Erziehungswissenschaft wird dieser Grund noch etwas häufiger genannt als im Durchschnitt aller Universitätsabsolventinnen und -absolventen (Abbildung 3.1). Die Masterquote kann also mit einigem Abstand zum Studium durchaus noch steigen. Keine Zulassung im gewünschten Studiengang bekommen zu haben, spielt demgegenüber fast keine Rolle. Nur etwa $10 \%$ derjenigen, die kein Masterstudium aufnehmen, geben dies als Grund an (Abbildung 3.1). Mehr als $40 \%$ sind nicht in ein Masterstudium gewechselt, weil sie keinen passenden Studiengang gefunden haben. Dies könnte mehrere Ursachen haben: noch vorhandene Angebotslücken bei den Masterstudiengängen in der Erziehungswissenschaft, häufig spezialisierte Angebote, die auf eine geringe Nachfrage treffen, oder eine geringere Mobilitätsbereitschaft, um den passenden Studiengang an einem anderen Standort zu belegen. Absolventenbefragungen geben jedoch keine Auskunft darüber, welche Studiengänge gesucht wurden. Auch finanzielle Gründe spielen in der Erziehungswissenschaft eine überdurchschnittlich große Rolle (Abbildung 3.1).

\section{Tätigkeiten nach dem Bachelorabschluss}

Etwa ein bis eineinhalb Jahre nach dem Studienabschluss befinden sich über alle Fächer hinweg noch mehr als zwei Drittel der Universitätsbachelors in einem weiterführenden (Master-)Studium, das teilweise durch Jobs finanziert wird (Tabelle 3.5). Ein Viertel ist mit mehr als der Hälfte eines Vollzeitäquivalents erwerbstätig, allenfalls zu einem kleineren Teil befindet sich diese Gruppe in einem berufsbegleitenden Masterstudium. Arbeitslosigkeit spielt mit 3 bis $5 \%$ kaum eine Rolle. Auch die Nicht-Erwerbstätigkeit aus sonstigen Gründen, insbesondere aufgrund von Kinderbetreuung, kommt nur selten vor. Insgesamt ist der Anteil der noch Studierenden des Abschlussjahrgangs 2013 zum Befragungszeitpunkt niedriger als der Anteil der Bachelors, die ein Masterstudium begonnen haben (vgl. Tabelle 3.4 und Tabelle 3.5). Dies kann auf die leicht steigende Zahl von Studienabbrüchen im Masterstudium hindeuten (zwischen 2010 und 2012 von 11 auf 15 \%, vgl. Heublein et al. 2017: 268). Kürzere und bereits beendete Masterstudiengänge spielen hingegen keine Rolle, denn bis zum Befragungszeitpunkt, ein bis eineinhalb Jahre nach dem Studienabschluss, kommen sie im Universitätsbereich kaum vor. 
Im Vergleich der Fachrichtungen ist die Erziehungswissenschaft mit über $40 \%$ durch einen überdurchschnittlich hohen Anteil an Erwerbstätigen gekennzeichnet. Nur im Sozialwesen ist der Anteil Erwerbstätiger mit fast 80 \% deutlich höher. Gut die Hälfte der Absolventinnen und Absolventen aus der Erziehungswissenschaft befindet sich noch im Masterstudium, weniger als nach einem universitären Bachelorabschluss insgesamt. Arbeitslosigkeit spielt für die Bachelors aus der Erziehungswissenschaft ebenso wie in den anderen Fachrichtungen kaum eine Rolle. Lediglich der Anteil derjenigen, die - überwiegend aus familiären Gründen - nicht erwerbstätig sind, liegt leicht über dem Durchschnitt. Die Suche nach einer ersten Anstellung nach dem Studium erfolgt in der Erziehungswissenschaft am schnellsten im Vergleich der ausgewiesenen Fachrichtungen; nach durchschnittlich drei Monaten haben sie eine Stelle gefunden (Tabelle 3.5).

Tabelle 3.5: Tätigkeiten ${ }^{1}$ ca. ein Jahr nach dem Bachelorabschluss 2013 und $2009^{2}$

\begin{tabular}{|c|c|c|c|c|c|c|c|c|}
\hline $\begin{array}{l}\text { Tätigkeiten ca. ein Jahr } \\
\text { nach Studienabschluss }\end{array}$ & 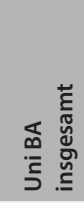 & 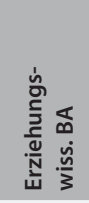 & 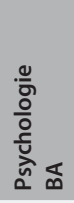 & 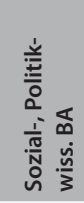 & 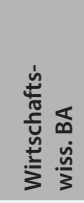 & 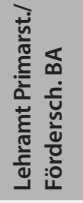 & 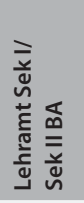 & 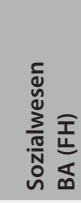 \\
\hline & \multicolumn{8}{|c|}{ (in \%) } \\
\hline Studium & 68 [73] & $53[67]$ & $84[/]$ & $71[81]$ & $65[74]$ & $54[-]$ & $81[-]$ & $21[33]$ \\
\hline erwerbstätig ${ }^{3}$ & 25 [19] & $44[33]$ & $12[/]$ & $23[12]$ & 28 [19] & $39[-]$ & $11[-]$ & $78[68]$ \\
\hline Jobben $^{4}$ & $21[27]$ & $22[27]$ & $24[/]$ & 28 [33] & $17[27]$ & $24[-]$ & $42[-]$ & $8[7]$ \\
\hline arbeitslos, stellensuchend & 4 [3] & $3[1]$ & $3[/]$ & $5[4]$ & 4 [3] & $3[-]$ & $2[-]$ & $2[3]$ \\
\hline \multirow[t]{2}{*}{$\begin{array}{l}\text { aus sonstigen Gründen } \\
\text { nicht erwerbstätig }\end{array}$} & $3[3]$ & $7[2]$ & $3[/]$ & 4 [2] & $2[4]$ & $6[-]$ & $2[-]$ & 6 [5] \\
\hline & \multicolumn{8}{|c|}{ (in Monaten) } \\
\hline $\begin{array}{l}\text { Dauer der Stellensuche bis } \\
\text { zur ersten Beschäftigung }\end{array}$ & 3,4 & 3,0 & 3,5 & 4,7 & 3,2 & / & / & 3,1 \\
\hline$n$ & $\begin{array}{r}7.325 \\
{[2.595]}\end{array}$ & $\begin{array}{r}396 \\
{[150]}\end{array}$ & $\begin{array}{l}285 \\
{[77]}\end{array}$ & $\begin{array}{r}499 \\
{[236]}\end{array}$ & $\begin{array}{r}897 \\
{[468]}\end{array}$ & $\begin{array}{r}289 \\
{[-]}\end{array}$ & $\begin{array}{r}191 \\
{[-]}\end{array}$ & $\begin{array}{r}469 \\
{[356]}\end{array}$ \\
\hline
\end{tabular}

1 Mehrfachnennungen möglich

2 Vergleichswert für Kohorte 2009 [in Klammern]

3 Befragte, die vorrangig erwerbstätig geworden sind; ohne studienbegleitend Erwerbstätige

4 Einschließlich Befragte, die studienbegleitend jobben

Quelle: DZHW-Absolventenpanel 2013.1 und 2009.1

Neben der Masterquote ist arbeitsmarkt- wie bildungspolitisch von Interesse, in welche Erwerbstätigkeiten Bachelors einmünden, die (zunächst) kein Masterstudium aufnehmen. Beides hängt insofern miteinander zusammen, 
als eine tendenziell unterwertige Beschäftigung von Bachelors, auf die verschiedene Analysen hindeuten (z.B. Autorengruppe Bildungsberichterstattung 2018, Tabelle F5-20web; Fabian/Hillmann/Trennt/Briedis 2016: 27ff.), das Interesse an einem Masterstudium verstärken dürfte. Allerdings wird in der ersten Zeit nach dem Bachelorabschluss mit Ausnahme der Erziehungswissenschaft und des Sozialwesens nur ein kleinerer Teil der Absolventinnen und Absolventen erwerbstätig (s. Tabelle 3.5), sodass die Basis für Auswertungen eher schmal ist. Hierfür stehen im Absolventenpanel 2013 zwei Kennzahlen zur Verfügung: die Adäquanz der Beschäftigung sowie das Einkommen.

Tabelle 3.6: Merkmale der Erwerbstätigkeit ${ }^{1}$ ca. ein Jahr nach dem Bachelorabschluss $2013^{2}$

\begin{tabular}{|c|c|c|c|c|c|c|}
\hline $\begin{array}{l}\text { Einschätzung der Adäquanz } \\
\text { der Beschäftigung: Welcher } \\
\text { Abschluss ist erforderlich für } \\
\text { die Erwerbstätigkeit }\end{array}$ & $\begin{array}{l}\text { Uni BA } \\
\text { insgesamt }\end{array}$ & $\begin{array}{l}\text { Erzie- } \\
\text { hungs- } \\
\text { wiss. BA }\end{array}$ & $\begin{array}{l}\text { Psycho- } \\
\text { logie BA }\end{array}$ & $\begin{array}{l}\text { Sozial-, } \\
\text { Politik- } \\
\text { wiss. BA }\end{array}$ & $\begin{array}{l}\text { Wirt- } \\
\text { schafts- } \\
\text { wiss. BA }\end{array}$ & $\begin{array}{l}\text { Sozial- } \\
\text { wesen } \\
\text { BA (FH) }\end{array}$ \\
\hline & \multicolumn{6}{|c|}{ (in \%) } \\
\hline höherer Abschluss & 20 & 7 & / & 18 & 22 & 8 \\
\hline mein Abschlussniveau & 49 & 50 & 1 & 48 & 52 & 72 \\
\hline $\begin{array}{l}\text { geringeres Abschlussniveau/kein } \\
\text { Hochschulabschluss erforderlich }\end{array}$ & 31 & 43 & / & 34 & 26 & 20 \\
\hline \multirow[t]{2}{*}{$n$ (Adäquanz) } & 1.588 & 162 & 32 & 104 & 234 & 345 \\
\hline & \multicolumn{6}{|c|}{ (in Euro) } \\
\hline Brutto-Monatseinkommen ${ }^{3}$ & 2.750 & 2.400 & I & 2.500 & 3.050 & 2.700 \\
\hline$n$ (Einkommen Vollzeit) & 776 & 77 & / & 54 & 161 & 205 \\
\hline
\end{tabular}

1 Nur Absolventinnen und Absolventen, die vorrangig erwerbstätig geworden sind; ohne Jobben und nachrangige, studienbegleitende Erwerbstätigkeiten

2 Ohne Lehramt, da hier kaum Absolventinnen und Absolventen in Erwerbstätigkeit übergehen

3 Nur Vollzeitbeschäftigte, inklusive monatlicher oder jährlicher Zulagen, die umgerechnet wurden

Quelle: DZHW-Absolventenpanel 2013.1

Die Adäquanz zwischen erworbener Qualifikation und Erwerbstätigkeit gilt in der Arbeitsmarktforschung als ein wesentlicher Indikator für die Beschäftigungsqualität. Adäquanz ist ein mehrdimensionales Konstrukt, das sich aus den Kriterien der Fach-, Positions-, Niveau- und Abschlussadäquanz zusammensetzt, welche wiederum zu einer vier- oder zweistufigen Skala (von vollbis überhaupt nicht adäquat) zusammengefasst werden können (Fehse/Kerst 2007; Wolter/Koepernik 2012: 298ff). Unabhängig vom Fach sieht sich etwa ein Drittel der universitären Bachelors unterhalb des eigenen Abschlussniveaus erwerbstätig, ein Fünftel schätzt die eigene Erwerbstätigkeit hingegen 
auf dem Masterniveau ein und die Hälfte sieht sich abschlussadäquat beschäftigt (Tabelle 3.6). In der Erziehungswissenschaft ist der Anteil der nicht abschlussadäquat Beschäftigten mit mehr als $40 \%$ unter den Vergleichsfächern am höchsten ${ }^{10}$, gefolgt von den Sozial- und Politikwissenschaften. Im Sozialwesen hingegen sehen sich 80 \% kurz nach dem Studium (mindestens) abschlussadäquat beschäftigt. Beim Einkommen weisen die Bachelors aus der Erziehungswissenschaft kurz nach dem Studium mit 2.400 Euro das geringste Bruttomonatseinkommen auf. Hier schlägt sich vermutlich der relativ hohe Anteil unterwertiger Beschäftigung nieder. Ähnlich dürfte es in den Sozial- und Politikwissenschaften sein.

\subsubsection{Verbleib nach dem Masterabschluss}

Nach dem Masterabschluss stehen die Absolventinnen und Absolventen vor einer weiteren Übergangsstelle, entweder in eine Erwerbstätigkeit oder weitere Bildungssequenzen (u.a. Promotion). Der größte Teil der Masterabsolventinnen und -absolventen wird nach dem Abschluss erwerbstätig (Tabelle 3.7); in der Erziehungswissenschaft sind es über $90 \%$. Arbeitslos oder stellensuchend ist nur ein sehr kleiner Teil (3\%). Auch Jobben oder weiteres Studium spielen kaum eine Rolle. Auch temporäre Nichterwerbstätigkeit ist nicht sehr häufig. Etwas anders ist dies in den Sozial- und Politikwissenschaften; hier ist ein größerer Anteil der Absolventinnen und Absolventen nur in Jobs oder sogar arbeitslos. Für die Einmündung in die Erwerbstätigkeit benötigen die Masterabsolventinnen und -absolventen aller Fächer mit durchschnittlich vier Monaten geringfügig mehr Zeit als nach dem Bachelorabschluss, wobei die durchschnittliche Suchdauer in der Erziehungswissenschaft und den Sozial-/Politikwissenschaften noch etwas länger dauert. Dies deutet auf eine etwas schwierigere Arbeitsmarktsituation hin.

Im Vergleich mit dem Abschlussjahrgang 2009 zeigt sich für die Erziehungswissenschaft in der Kohorte 2013 eine weitgehend gleiche Verteilung auf die verschiedenen Tätigkeiten (Tabelle 3.7). Ähnlich ist es bei den Lehrerinnen und Lehrern, die sich zum Befragungszeitpunkt überwiegend im Referendariat befinden. Für die sozial- und wirtschaftswissenschaftlichen Absolventinnen und Absolventen scheint die Berufseinmündung für die Kohorte 2009 hingegen etwas schwieriger verlaufen zu sein als 2013. Der Anteil der jobbenden oder arbeitslosen Absolventinnen und Absolventen ist für den Abschlussjahrgang 2009 etwa ein Jahr nach dem Studienabschluss höher, möglicherweise eine Folge der Wirtschafts- und Finanzkrise 2008/09.

10 Diese Befragten geben als Berufsbezeichnung in vielen Fällen Erzieherin, Kindheitspädagogin oder Helferin in Kindertageseinrichtungen bzw. Jugendeinrichtungen an. 
Tabelle 3.7: Tätigkeiten ${ }^{1}$ ca. ein Jahr nach dem Masterabschluss 2013 (im Vergleich mit dem Diplom- und Magisterabschluss 2009) ${ }^{2}$

\begin{tabular}{|c|c|c|c|c|c|c|c|c|}
\hline $\begin{array}{l}\text { Tätigkeiten ca. ein Jahr } \\
\text { nach Studienabschluss }\end{array}$ & 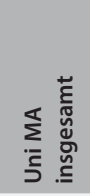 & 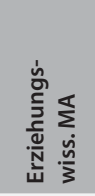 & 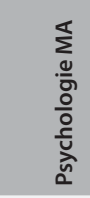 & 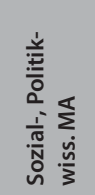 & 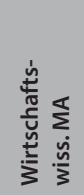 & 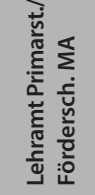 & 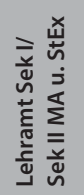 & 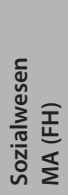 \\
\hline & \multicolumn{8}{|c|}{ (in \%) } \\
\hline erwerbstätig ${ }^{3}$ & $85[82]$ & $91[90]$ & $81[74]$ & $87[76]$ & $93[84]$ & $86[90]$ & 95 & / [88] \\
\hline Promotion & $25[26]$ & $16[5]$ & $15[25]$ & $22[19]$ & $12[12]$ & $3[1]$ & 5 & $/[1]$ \\
\hline Studium & $3[5]$ & $4[2]$ & $3[9]$ & $1[4]$ & $1[3]$ & $3[6]$ & 5 & $/[10]$ \\
\hline Jobben $^{4}$ & $4[7]$ & $3[7]$ & $5[5]$ & $10[14]$ & $1[6]$ & $3[4]$ & 4 & $/[4]$ \\
\hline arbeitslos, stellensuchend & $5[7]$ & $4[1]$ & $3[4]$ & $9[12]$ & $3[10]$ & $3[1]$ & 1 & $/[3]$ \\
\hline \multirow[t]{2}{*}{$\begin{array}{l}\text { aus sonstigen Gründen } \\
\text { nicht erwerbstätig }\end{array}$} & $4[4]$ & $5[5]$ & $9[7]$ & $2[3]$ & $1[2]$ & $4[5]$ & 5 & $/[7]$ \\
\hline & \multicolumn{8}{|c|}{ (in Monaten) } \\
\hline $\begin{array}{l}\text { Dauer der Stellensuche bis } \\
\text { zur ersten Beschäftigung }^{5}\end{array}$ & 4,0 & 5,0 & 3,8 & 6,0 & 3,8 & 3,2 & 2,8 & I \\
\hline$n$ & $\begin{array}{r}3.433 \\
{[2.581]}\end{array}$ & $\begin{array}{r}127 \\
{[206]}\end{array}$ & $\begin{array}{r}145 \\
{[159]}\end{array}$ & $\begin{array}{r}213 \\
{[189]}\end{array}$ & $\begin{array}{r}557 \\
{[513]}\end{array}$ & $\begin{array}{r}847 \\
{[389]}\end{array}$ & $\begin{array}{r}448 \\
{[548]}\end{array}$ & $\begin{array}{r}44 \\
{[216]}\end{array}$ \\
\hline
\end{tabular}

1 Mehrfachnennung möglich

2 Vergleichswerte für Diplom- und Magisterabschlüsse aus dem Abschlussjahrgang 2009 in [eckigen Klammern]

3 Befragte, die vorrangig erwerbstätig geworden sind; ohne studienbegleitend Erwerbstätige

4 Einschließlich Befragte, die studienbegleitend jobben

5 Vergleichbare Auswertung für das Absolventenpanel 2009 nicht möglich

Quelle: DZHW-Absolventenpanel 2013.1 und 2009.1

Wie bei den Absolventinnen und Absolventen mit Bachelorabschluss können auch für die Masterabsolventinnen und -absolventen des Abschlussjahrgangs 2013 die Adäquanz der Erwerbstätigkeit und das Einkommen als Merkmale der Erwerbstätigkeit betrachtet werden (Tabelle 3.8). ${ }^{11}$ Insgesamt ähneln die Ergebnisse unabhängig vom Studienfach denen nach dem Bachelorabschluss, wobei Master mit einem universitären Abschluss etwa ein Jahr nach dem Studienabschluss mit knapp $78 \% \mathrm{zu}$ einem höheren Anteil abschlussadäquat beschäftigt werden als Bachelors (69 \%, vgl. Tabelle 3.6). Dieser Anstieg um etwa zehn Prozentpunkte findet sich auch in der Erziehungswissenschaft (von 57 auf $67 \%$ ), wo sich jedoch mit einem Masterabschluss immer noch etwa ein Drittel unterhalb des eigenen Abschlussniveaus beschäftigt sieht. Master aus den Sozial- und Politikwissenschaften (71\%) und den Wirtschaftswissenschaften $(76 \%)$ kommen auf etwas höhere Adäquanzwerte. Mit deutlichem

11 Ein Vergleich mit dem Abschlussjahrgang 2009 ist nicht möglich, da die Adäquanz mit einem anderen Instrument erhoben wurde. 
Abstand dazu liegt der Anteil adäquat Beschäftigter in der Psychologie und in den Lehrämtern (überwiegend im Referendariat) bei über $90 \%$.

Tabelle 3.8: Merkmale der Erwerbstätigkeit ${ }^{1}$ ca. ein Jahr nach dem Masterabschluss $2013^{2}$ (Einkommen auch für Jahrgang 2009)

\begin{tabular}{|c|c|c|c|c|c|c|c|}
\hline $\begin{array}{l}\text { Einschätzung der Adäquanz } \\
\text { der Beschäftigung: Welcher } \\
\text { Abschluss ist erforderlich für } \\
\text { die Erwerbstätigkeit? }^{3}\end{array}$ & 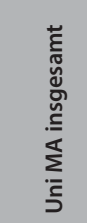 & 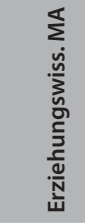 & 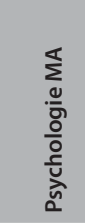 & 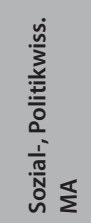 & 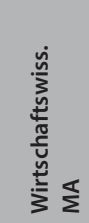 & 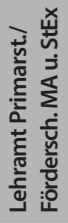 & 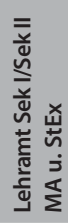 \\
\hline & \multicolumn{7}{|c|}{ (in \%) } \\
\hline höherer Abschluss & 4 & 3 & 5 & 4 & 2 & 6 & 1 \\
\hline mein Abschlussniveau & 74 & 64 & 86 & 67 & 74 & 86 & 91 \\
\hline geringeres Abschlussniveau & 14 & 20 & 6 & 12 & 17 & 5 & 3 \\
\hline $\begin{array}{l}\text { kein Hochschulabschluss } \\
\text { erforderlich }\end{array}$ & 8 & 14 & 3 & 17 & 7 & 4 & 5 \\
\hline \multirow[t]{2}{*}{ n (Adäquanz) } & 2.600 & 100 & 96 & 154 & 475 & 543 & 399 \\
\hline & \multicolumn{7}{|c|}{ (in Euro) } \\
\hline Brutto-Monatseinkommen ${ }^{4}$ & $\begin{array}{r}3.400 \\
{[3.000]}\end{array}$ & $\begin{array}{r}2.850 \\
{[2.400]}\end{array}$ & $\begin{array}{r}3.250 \\
{[2.600]}\end{array}$ & $\begin{array}{r}2.900 \\
{[2.700]}\end{array}$ & $\begin{array}{r}3.800 \\
{[3.200]}\end{array}$ & / & / \\
\hline n (Einkommen Vollzeit) & $\begin{array}{r}1.828 \\
{[1.025]}\end{array}$ & $\begin{array}{r}57 \\
{[101]}\end{array}$ & $\begin{array}{r}51 \\
{[53]}\end{array}$ & $\begin{array}{r}97 \\
{[71]}\end{array}$ & $\begin{array}{r}415 \\
{[257]}\end{array}$ & l & I \\
\hline
\end{tabular}

1 Nur Absolventinnen und Absolventen, die vorrangig erwerbstätig geworden sind; ohne Jobben und nachrangige, studienbegleitende Erwerbstätigkeiten

2 Ohne MA Sozialwesen, da aufgrund der geringen Übergangsquote in das Masterstudium nicht genügend Masterabschlüsse in der Stichprobe vertreten sind $(n=44)$. Einkommen ohne Lehramt, da die Einkommen im Referendariat nicht mit denen in den anderen Fachrichtungen vergleichbar sind.

3 Die Kategorien "geringeres Abschlussniveau « und »kein Hochschulabschluss erforderlich« können hier, anders als in Tabelle 3.6, getrennt ausgewiesen werden. »Geringeres Abschlussniveau " verweist auf Tätigkeiten, die nach Einschätzung der Befragten einen Abschluss auf Bachelorniveau erfordern.

4 Nur Vollzeitbeschäftigte, inklusive monatlicher oder jährlicher Zulagen

Quelle: DZHW-Absolventenpanel 2013.1 und 2009.1

Beim Einkommen (nur Vollzeit-Erwerbstätige) liegen Master der Erziehungs-, Sozial- und Politikwissenschaften um etwa $15 \%$ unter dem Durchschnitt von 3.400 Euro, der vor allem durch die relativ hohen Einkommen in den Wirtschafts- und Ingenieurwissenschaften (hier nicht ausgewiesen) beeinflusst wird. Auch beim Abschlussjahrgang 2009 erzielten die Absolventinnen und Absolventen mit Magister- oder Diplomabschluss in der Erziehungswissenschaft ein Einkommen, das um 20 \% unter dem Durchschnitt aller universitären Diplom- und Magisterabschlüsse liegt (Tabelle 3.8). 
Der Übergang in die Promotion ist ein wichtiger Indikator für das zur Verfügung stehende Potenzial des wissenschaftlichen Nachwuchses in einem Fach, auch wenn nicht alle Promovierten am Ende eine wissenschaftliche Laufbahn einschlagen. Über alle Fächer hat etwa ein Jahr nach dem Masterabschluss im Prüfungsjahr 2013 ein Viertel der Absolventinnen und Absolventen eine Promotion aufgenommen, wobei sich die Fachrichtungen stark unterscheiden (Tabelle 3.7). Nach dem Lehramtsabschluss ist der Übergang in die Promotion sehr selten. Unter den hier betrachteten Vergleichsfächern ist er in den Sozial- und Politikwissenschaften mit $22 \%$ am höchsten. In der Erziehungswissenschaft liegt die Übergangsquote in die Promotion etwa zehn Prozentpunkte unter dem Durchschnitt an den Universitäten (16 vs. 25 \%). Die deutlich geringere Promotionsintensität (siehe nächster Absatz) deutet aber darauf hin, dass ein hoher Anteil der Promotionsanfängerinnen und -anfänger die Promotion wieder aufgibt. Verglichen mit dem Jahrgang 2009, als nur 5 \% eine Promotion aufgenommen hatten, ist der Anteil der in eine Promotion übergehenden Absolventinnen und Absolventen in der Erziehungswissenschaft beim Abschlussjahrgang 2013 jedoch deutlich gestiegen. Ob dies mit einer Ausweitung strukturierter Promotionsprogramme oder dem Ausbau der empirischen Bildungsforschung und dem damit verbundenen höheren Drittmittelzufluss zusammenhängt, muss hier offenbleiben.

Der geringere Übergang in eine Promotion spiegelt sich auch in der Promotionsintensität wider (Tabelle 3.9). Insgesamt schließen etwa $16 \%$ aller promotionsberechtigten Universitätsabsolventinnen und -absolventen (ohne Medizin) ein Promotionsstudium ab. In der Erziehungswissenschaft fällt die Promotionsintensität mit 5 bis $6 \%$ am geringsten aus; seit 2010 zeigt sich eine leicht sinkende Tendenz. In den Sozial- und Politikwissenschaften erreicht die Promotionsquote ein annähernd dreimal so hohes Niveau, in den Wirtschaftswissenschaften liegt sie doppelt so hoch.

Mit den Daten des 2013 gestarteten Projekts »Karrieren Promovierter «, in dem eine Vollerhebung der Promovierten des Abschlussjahres 2014 als Panelbefragung mit bisher vier Wellen realisiert wurde, können verschiedene Merkmale zur Promotion und zur Situation nach der Promotion im Fächervergleich untersucht werden. ${ }^{12}$ Dabei zeigt sich für die Promotionen in der Erziehungswissenschaft (hier mit Sonderpädagogik): Ein höherer Anteil der erziehungswissenschaftlichen Promotionen des Jahrgangs 2014 wurde auf Haushaltsstellen ( 28 vs. $21 \%$ ) und als freie Promotionen (38 vs. $34 \%$ ) erstellt, während Promotionen auf Drittmittelstellen seltener vorkamen (20 vs. $29 \%$ ). Nur $11 \%$ der Promovierten hatten sich während der Promotion mindestens einmal in der Woche mit der Hauptbetreuerin bzw. dem Hauptbetreuer ausgetauscht

12 Vgl. https://www.dzhw.eu/pdf/22/ergebnisse_erziehungswissenschaften_promoviertenstudie.pdf [Zugriff: 27.7.2019]. In der Auswertung werden Erziehungswissenschaft und Sonderpädagogik gemeinsam dargestellt. Die Auswertung für die ersten beiden Wellen des Promoviertenpanels basiert auf 175 (1. Welle) bzw. 108 (2. Welle) Promotionen in den Fächern Erziehungswissenschaft und Sonderpädagogik. 
(vs. $27 \%$ insgesamt). $39 \%$ hatten nur einmal im Semester oder seltener Kontakt (vs. 32 \%). Promovierende des Jahrgangs 2014 waren in der Erziehungswissenschaft mit $9 \%$ unterdurchschnittlich häufig zu einem Forschungsaufenthalt im Ausland (vs. 14 \% insgesamt). In den Geistes- (32\%) und den Sozialwissenschaften $(30 \%)$ kam das noch deutlich häufiger vor.

Tabelle 3.9: Promotionsintensität ${ }^{1}$ insgesamt und in den Vergleichsfächern 1993 bis 2017 (in \%)

\begin{tabular}{|c|c|c|c|c|c|c|c|c|c|c|c|c|}
\hline & ू̆ & $\stackrel{\text { ू }}{\circ}$ & ষ্ণ & ๕̊ & $\stackrel{2}{\frac{2}{2}}$ & $\bar{i}$ & $\overbrace{}^{2}$ & $\bar{m}^{m}$ & $\stackrel{\text { i }}{+}$ & $\stackrel{n}{\frac{n}{2}}$ & $\stackrel{\circ}{2}$ & $\hat{\bar{i}}$ \\
\hline $\begin{array}{l}\text { insgesamt } \\
\text { über alle Fächer }\end{array}$ & - & - & 19,9 & 24,0 & 19,7 & 19,2 & 18,4 & 18,3 & 19,3 & 20,2 & 20,5 & 19,8 \\
\hline $\begin{array}{l}\text { ohne Medizin u. } \\
\text { Gesundheitswiss. }\end{array}$ & - & - & 14,7 & 18,2 & 15,4 & 14,9 & 14,6 & 15,0 & 15,7 & 16,6 & 16,8 & 16,3 \\
\hline $\begin{array}{l}\text { Rechts-, Wirtschafts- und } \\
\text { Sozialwissenschaften }\end{array}$ & - & - & 8,8 & 12,0 & 9,5 & 9,1 & 9,2 & 9,2 & 9,6 & 10,2 & 10,7 & 9,9 \\
\hline $\begin{array}{l}\text { Erziehungs- } \\
\text { wissenschaft }\end{array}$ & - & - & 5,8 & 6,2 & 6,9 & 6,4 & 5,9 & 5,1 & 5,8 & 5,9 & 4,9 & 5,5 \\
\hline Psychologie & - & - & 12,9 & 19,0 & 15,2 & 14,8 & 16,4 & 17,0 & 16,3 & 16,3 & 18,3 & 16,3 \\
\hline $\begin{array}{l}\text { Sozial-/Politikwissen- } \\
\text { schaften }\end{array}$ & - & - & 17,7 & 19,5 & 10,7 & 10,4 & 9,6 & 9,7 & 10,8 & 11,5 & 13,1 & 15,3 \\
\hline $\begin{array}{l}\text { Wirtschafts- } \\
\text { wissenschaften }\end{array}$ & - & 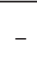 & 5,8 & 10,3 & 7,4 & 7,5 & 7,6 & 8,1 & 8,3 & 9,6 & 10,2 & 8,9 \\
\hline
\end{tabular}

1 Die Promotionsintensität wird errechnet als Anteil der Promovierten eines Jahres an der Zahl der sog. Bezugsabsolventen vier Jahre zuvor (etwa vier Jahre werden als durchschnittliche Promotionsdauer veranschlagt), vgl. Konsortium Bundesbericht wissenschaftlicher Nachwuchs 2017: 154. Bezugsabsolventen sind Erstabsolventinnen und -absolventen mit traditionellen Abschlüssen (Diplom, Magister, künstlerische Abschlüsse, Staatsexamen) oder mit Masterabschluss (einschl. Lehramt); die Möglichkeit, direkt nach dem Bachelorabschluss in eine Promotion überzugehen, bleibt hier außer Acht.

Quelle: Statistisches Bundesamt, Hochschulstatistik, Berechnung der hier nicht ausgewiesenen Bezugsabsolventen durch Recherche in HIS/ICE, eigene Berechnungen

Überdurchschnittlich viele der promovierten Erziehungswissenschaftlerinnen und Erziehungswissenschaftler beabsichtigen ein Jahr nach der Promotion, dauerhaft in der Wissenschaft zu bleiben (42\%). Das sind ebenso viele wie in den Geisteswissenschaften, aber mehr als in den Sozial- (35\%) und Wirtschaftswissenschaften (26\%), im Durchschnitt sind es $22 \%$. Zwei Jahre nach der Promotion sind dann in der Erziehungswissenschaft $51 \%$ tatsächlich in der Wissenschaft tätig (über alle Fächer 33 \%), etwa ebenso viele wie in den Geistes- und Sozialwissenschaften (54\%). Beim Einkommen liegen die promovierten Erziehungswissenschaftlerinnen und -wissenschaftler jedoch am unteren Ende der Skala. Sie erzielen 3.800 Euro Monatseinkommen und damit deutlich 
weniger als durchschnittlich (4.500 Euro). Möglicherweise deutet der hohe Verbleib der Promovierten aus der Erziehungswissenschaft im Wissenschaftssystem darauf hin, dass aufgrund der geringen Promotionsintensität besonders viele Promovierte eine Chance haben, eine wissenschaftliche Laufbahn einzuschlagen. Darüber hinaus sind die außerwissenschaftlichen Arbeitsmarktsegmente für Promovierte aus der Erziehungswissenschaft im Vergleich etwa zu den breiteren Tätigkeitsfeldern in den Sozialwissenschaften eng begrenzt.

\subsection{Merkmale der Erwerbstätigkeit in mittelfristiger Perspektive (fünf bis zehn Jahre nach Studienabschluss)}

Bereits mehrfach, zuletzt im Datenreport Erziehungswissenschaft 2016, wurde dargestellt, dass der mittel- und langfristige Verbleib der Absolventinnen und Absolventen erziehungswissenschaftlicher Studiengänge und der Vergleichsfächer nicht unmittelbar aus der Situation kurz nach dem Studienabschluss abgeleitet werden kann. Langfristig ist etwa die Erwerbsbeteiligung in der Erziehungswissenschaft nicht wesentlich geringer als in den Vergleichsfächern (Kerst/Wolter/Züchner 2016: 110ff.). Für andere Indikatoren, namentlich Einkommen und Beschäftigungsadäquanz, stellt sich die Situation auch mittelfristig und langfristig als weniger günstig dar, »die sich allerding bei keinem Indikator zu einer Art Prekarisierung der Beschäftigungsverhältnisse ... verfestigt« (ebd.: 133). Als Datenbasis für frühere Darstellungen diente eine Verbleibsstudie aus den 1990er Jahren (vgl. Krüger u.a. 2003; Rauschenbach/ Züchner 2004), die allerdings nicht fächervergleichend angelegt war. Für spätere Absolventenkohorten wurde in den Datenreporten das Absolventenpanel des DZHW verwendet (siehe Abschnitt 3.2.1). Dieser Datenbestand wird auch im Folgenden genutzt, ergänzt um weitere Kohorten und Befragungswellen, die seit dem Datenreport 2016 hinzugekommen sind. ${ }^{13}$ Wie schon in Abschnitt 3.2.1 ausgeführt, stellen mittel- und langfristige Verbleibsinformationen zu Absolventinnen und Absolventen mit Bachelor- oder Masterabschluss noch eine Datenlücke dar.

Für bis zu zehn Jahre nach dem Studienabschluss stehen in den hier verwendeten Daten monatsgenau Informationen über die ausgeübten Tätigkeiten zur Verfügung. Damit kann für jede Absolventenkohorte dargestellt werden,

13 Im Datenreport 2016 standen Verbleibsdaten der Absolventenkohorten 1997 und 2001 über zehn Jahre zur Verfügung, außerdem Daten der Kohorte 2005 nach fünf Jahren. In diesem Datenreport kommen die Zehnjahresdaten der Kohorte 2005 sowie die Fünfjahresdaten der Kohorte 2009 hinzu. Für die Erziehungswissenschaft stehen für die Kohorte 1997 Angaben von $\mathrm{n}=147$ (Welle 1), $\mathrm{n}=99$ (Welle 2) sowie $\mathrm{n}=90$ (Welle 3) Befragten zur Verfügung; für die Kohorte 2001 sind es $n=251$ (W1), $n=184$ (W2) bzw. $n=159$ (W3), für die Kohorte 2005 sind es $n=237$ (W1), n=148 (W2) sowie $n=106$ (W3), für die Kohorte $2009 n=206$ (W1) und $\mathrm{n}=106(\mathrm{~W} 2)$. 
wie viele Absolventinnen und Absolventen zu welchem Zeitpunkt erwerbstätig sind, welche Tätigkeiten sie ausüben und wie hoch der Anteil der in Elternzeit befindlichen Personen ist (Abbildung 3.2a und 3.2b). ${ }^{14}$ Hier zeigen sich die methodischen Vorteile eines Kohorten-/Längsschnittdesigns. Erkennbar werden Unterschiede zwischen den Kohorten, aber auch den Fachrichtungen. In allen hier einbezogenen Vergleichsfächern steigt die Erwerbsbeteiligung nach dem Studienabschluss rasch an und erreicht nach 12 bis 24 Monaten ein Niveau von 70 bis $90 \%$. Zugleich nimmt der Anteil der Personen mit Übergangsjobs deutlich ab, die mittel- und langfristig nur noch eine marginale Rolle spielen (in Abbildung 3.2a nur für die Erziehungswissenschaft dargestellt). Ab dem vierten oder fünften Berufsjahr zeigt sich dann ein steigender Anteil an Personen in Elternzeit (Abbildung 3.2b), insbesondere in Fächern mit einem hohen Anteil an Absolventinnen.

Unterschiede zwischen den Kohorten gibt es vor allem in der ersten Zeit nach dem Studienabschluss. Je nach gesamtwirtschaftlicher Lage, zusätzlich möglicherweise auch durch fachspezifische Faktoren beeinflusst, gelingt der Übergang unterschiedlich schnell. Auch das insgesamt erreichte Niveau der Erwerbsbeteiligung variiert etwas; am höchsten ist es in den Wirtschaftswissenschaften. Hier verlaufen die Kurven der Kohorten außerdem, wie im Sozialwesen, sehr ähnlich. In den Sozial- und Politikwissenschaften und der Erziehungswissenschaft gibt es größere Unterschiede zwischen den Kohorten. In der Erziehungswissenschaft etwa sind die Kohorten 1997 und 2005 langsamer in Erwerbstätigkeit gelangt als die Kohorten 2001 und 2009. Etwa zwei Jahre nach dem Abschluss haben dann jedoch alle vier Kohorten eine Erwerbsbeteiligung von etwa $80 \%$ erreicht. In den folgenden vier Jahren bleiben dann die Verlaufskurven für die Erziehungswissenschaft stabil; sechs Jahre nach dem Abschluss beginnen sie sich wieder etwas auseinanderzuentwickeln. So liegt die Erwerbsbeteiligung der Kohorte 2005 in dieser Zeit etwa zehn Prozentpunkte unter der der Kohorte 1997. Dies korrespondiert mit einem höheren Anteil an Personen in Elternzeit. Ergänzend wurde geprüft, ob hier möglicherweise alternative Erwerbsformen wie Werkverträge oder Jobs eine größere Bedeutung haben. Während Jobs langfristig keine Rolle spielen, liegt der Anteil der Erwerbstätigen mit Werkvertrag in den Kohorten 2001 und 2005 deutlich höher als in der Kohorte 1997. Nach sechs Jahren geben 10 bis 15 \% eine Erwerbstätigkeit im Rahmen von Werkverträgen an. Möglicherweise erfolgt ein Wiedereinstieg nach Elternzeit häufiger über Werkverträge. Arbeitslosigkeit kommt bei den befragten Absolventinnen und Absolventen kaum vor (vgl. dazu auch Autorengruppe Bildungsberichterstattung 2018: 200). Lediglich in der ersten Welle gibt es eine etwas höhere Sucharbeitslosigkeit (2005: 5 \%), die in den Folgewellen jedoch auf etwa $1 \%$ zurückgeht. Dabei unterscheiden sich die Vergleichsfächer kaum.

14 Auf die Darstellung weiterer Tätigkeiten wie Studium und Promotion, Praktika oder Ausbildungsverhältnisse wird hier verzichtet. 
Das Lehramt mit insgesamt sehr ähnlichen Verläufen zwischen den Kohorten unterscheidet sich von den anderen Fächern hauptsächlich durch die zweite Ausbildungsphase.

Abbildung 3.2a: Verlauf von Erwerbstätigkeit ${ }^{1}$, Elternzeit, Werkverträgen und Jobs in den ersten zehn Jahren nach dem Studienabschluss in der Erziehungswissenschaft (nur traditionelle Abschlüsse, in \%)
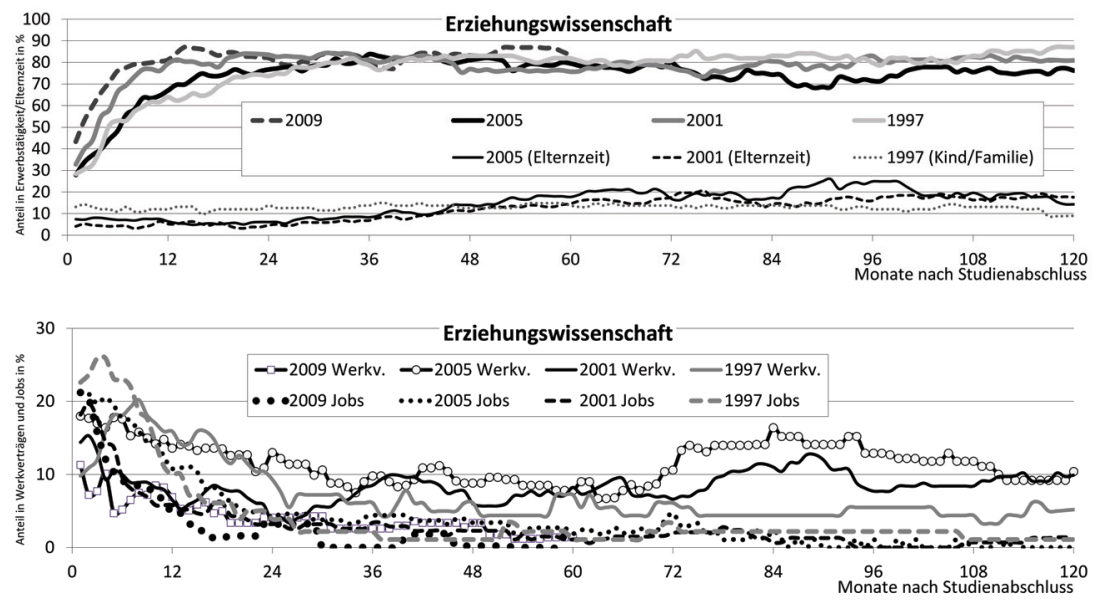

Abbildung 3.2b: Verlauf von Erwerbstätigkeit ${ }^{1}$ und Elternzeit in den ersten zehn Jahren nach dem Studienabschluss in den Vergleichsfächern ${ }^{2}$ (nur traditionelle Abschlüsse, in \%)
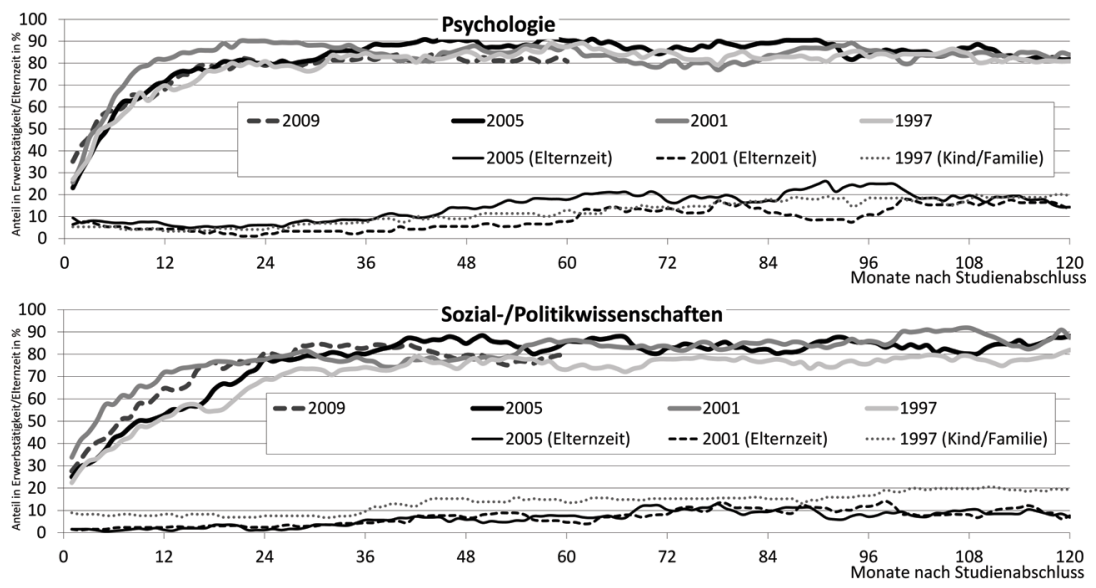
Studienabschlüsse und Verbleib
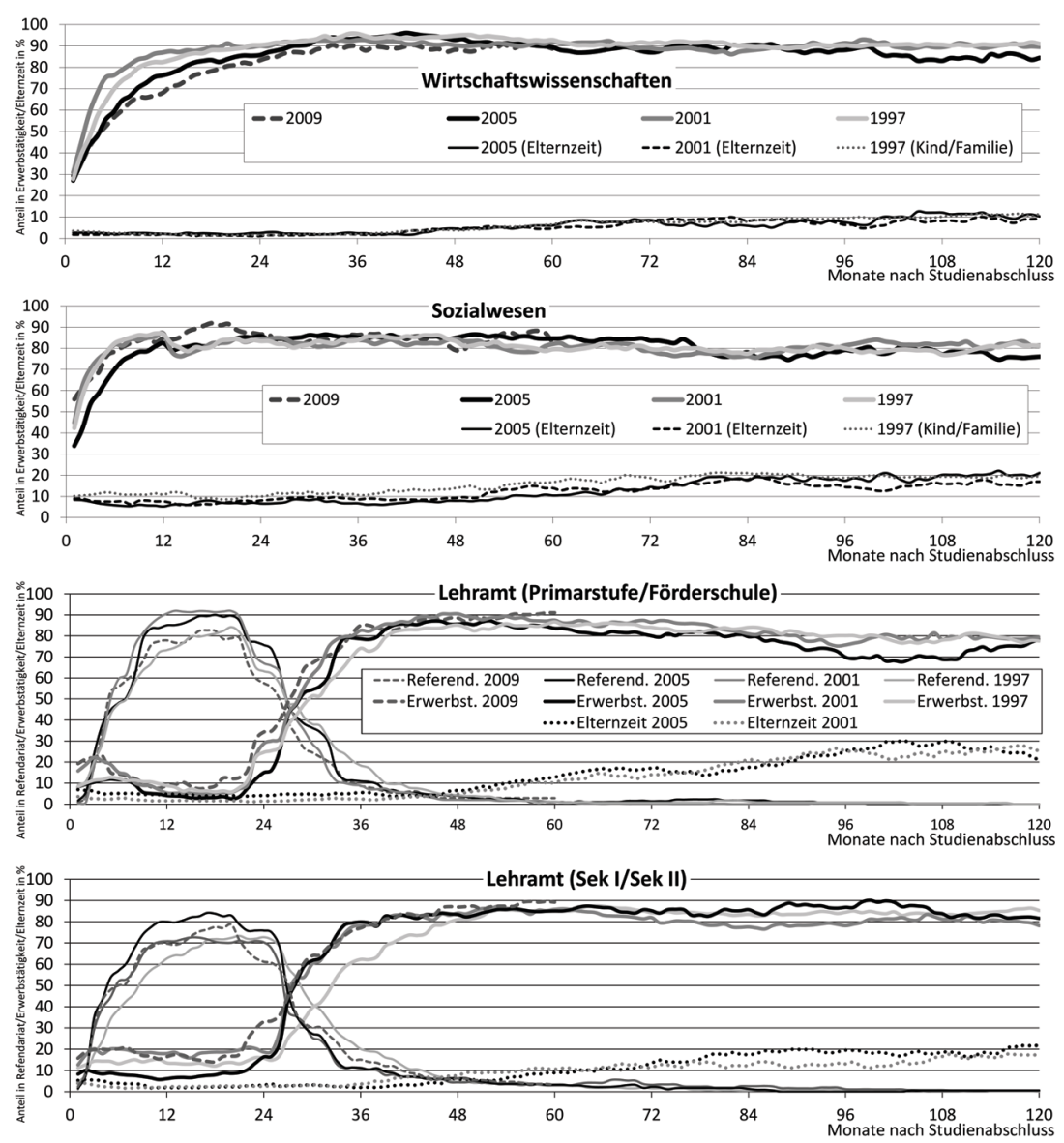

1 Selbstständige und nicht-selbstständige Erwerbstätigkeit, ohne Jobben und Werk-/ Honorarverträge

2 Für Sozialwesen wird das Anerkennungspraktikum als Erwerbstätigkeit gefasst.

Quelle: DZHW-Absolventenpanel 1997, 2001, 2005 und 2009

In den folgenden Ausführungen sollen verschiedene Merkmale der Erwerbstätigkeit, insbesondere für den Berufserfolg in mittel- und langfristiger Perspektive, dargestellt werden. Gibt es Hinweise darauf, dass Absolventinnen und Absolventen der Erziehungswissenschaft weniger Berufserfolg haben als jene aus den Vergleichsfächern? Berufserfolg wird hier als ein mehrdimensionales Konstrukt verstanden, für das verschiedene Indikatoren herangezogen werden. Diese können sowohl auf eher subjektiven Einschätzungen beruhen (z.B. Adäquanz, Zufriedenheit) als auch eher objektive Merkmale beschreiben (z.B. 
Einkommen oder die berufliche Stellung). Darüber hinaus wird dargestellt, in welchen Wirtschaftszweigen die Absolventinnen und Absolventen tätig werden, für welche Beschäftigungssektoren die Hochschulen also ausbilden und welche Bedeutung dabei eine Beschäftigung im öffentlichen Dienst hat. Auch wenn individuelle und gesellschaftliche Bildungserträge unterschiedlich ausfallen können, so ist ein individueller Berufserfolg doch zumindest indirekt auch ein Hinweis darauf, ob sich Hochschulbildung gesellschaftlich »auszahlt«.

\section{Adäquate Beschäftigung}

Der Anteil vertikal adäquat Beschäftigter ${ }^{15}$ variiert zwischen den Vergleichsfächern. Am höchsten ist die Beschäftigungsadäquanz im Lehramt, wo der Studienabschluss obligatorische Voraussetzung für die Beschäftigung ist, sowie in der Psychologie. Hier ist die Adäquanz bereits kurz nach dem Studium relativ hoch (Abbildung 3.3), steigt dann noch leicht an und bleibt in den folgenden Erhebungen konstant auf einem sehr hohen Niveau. Auch die Kohorten unterscheiden sich kaum. In der Erziehungswissenschaft sowie in den Sozial-, Politik- und Wirtschaftswissenschaften gibt es nach dem Studienabschluss einen zunächst geringeren, in den Folgewellen steigenden Anteil vertikal adäquat Beschäftigter. In diesen Fachrichtungen unterscheiden sich auch die Kohorten stärker, die offensichtlich unterschiedlich gute Bedingungen des Berufseinstiegs finden, was sich in der Beschäftigungsadäquanz niederschlägt. In der Erziehungswissenschaft steigt die Adäquanz in der Kohorte 2001 nach einem guten Berufseinstieg nur noch wenig an, während in den Kohorten 2005 und 1997 ein kontinuierlicher Zuwachs erfolgt. Beim Jahrgang 2005 ist die Beschäftigungsadäquanz nach zehn Jahren ähnlich hoch wie in den Vergleichsfächern Sozial-, Politik- und Wirtschaftswissenschaften. Für die Kohorte 2009 fällt die Adäquanz in der subjektiven Einschätzung in den ersten fünf Jahren noch einmal höher aus. Die sehr schnelle Einmündung in den Arbeitsmarkt (Abbildung 3.2a) hat hier offenbar einen höheren Anteil als in vorherigen Kohorten in eine adäquate Beschäftigung geführt. Die Finanz- und Wirtschaftskrise hat die Erziehungswissenschaft scheinbar weniger als andere Fächer getroffen. Multivariate Analysen (hier nicht dokumentiert) deuten darauf hin, dass es vor allem ein bereits adäquater, erfolgreicher Berufseinstieg ist, der zu höheren Adäquanzwerten auch in den folgenden Wellen führt (vgl. dazu bereits Fehse/Kerst 2007). Die Adäquanz der ersten Stelle wiederum wird neben der Abschlussnote des Studiums auch durch Praxiskontakte und fachnahe Erwerbstätigkeit während des Studiums positiv beeinflusst (ebd.).

15 Vertikale Adäquanz wird von der horizontalen Adäquanz (= Fachadäquanz) unterschieden und beinhaltet die subjektiv erhobene Einschätzung der Bedeutung des Hochschulabschlusses für die Erwerbstätigkeit sowie des Niveaus der beruflichen Position und der Aufgaben; vgl. dazu Fehse/Kerst 2007. 
Abbildung 3.3: Anteil vertikal adäquat Beschäftigter ${ }^{1}$ ein, fünf und zehn Jahre nach dem Studienabschluss, nach Fachrichtungen und Abschlusskohorten (nur traditionelle Abschlüsse, in \%)

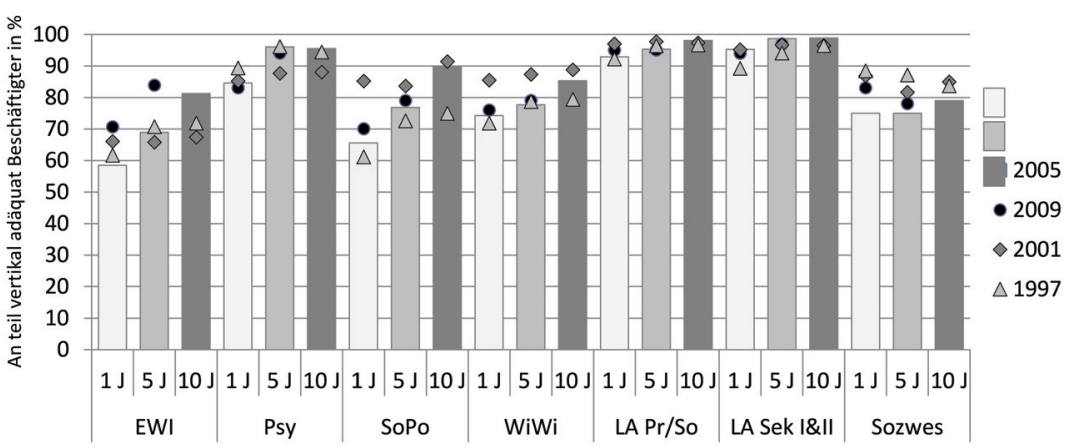

1 Subjektiv erhobene Einschätzung der Beschäftigungsadäquanz; zum Erhebungsinstrument und zur Bildung der Kennzahl vgl. Fehse/Kerst 2007.

Quelle: DZHW-Absolventenpanel 1997, 2001, 2005 und 2009

\section{Berufliche Stellung}

Bei der beruflichen Stellung spielt in der Erziehungswissenschaft, wie in den Vergleichsfächern, formal unterqualifizierte Beschäftigung (als Facharbeiter oder ungelernte Arbeiter codiert) nur eine geringe Rolle (Abbildung 3.4), in erster Linie in Form eines überdurchschnittlichen Anteils in qualifizierten Angestelltenpositionen, für die formal kein Universitätsabschluss erforderlich ist. Für 1997 und 2005, die Kohorten mit dem schwierigeren Einstieg, verändert sich dies auch in der dritten Welle nicht, für die Kohorte 2001 nimmt dieser Anteil mit den Wellen ab. Dieses Muster findet sich auch in den Sozial- und Politikwissenschaften. Der Anteil derjenigen in leitenden Positionen liegt in der Erziehungswissenschaft nach zehn Jahren mit etwa einem Drittel leicht über Durchschnitt für alle Fächer; hier unterscheiden sich die Kohorten kaum. In der Erziehungswissenschaft ist sogar bereits kurz nach dem Studienabschluss ein überdurchschnittlicher Anteil in leitenden Positionen zu finden. Der Anteil an Selbstständigen und Freiberuflern schwankt zwischen den Kohorten relativ stark. Im Absolventenjahrgang 2009 gibt es nach dem Studienabschluss kaum Selbstständige, während es 1997 (15\%) und 2005 (21\%) deutlich mehr waren. Zehn Jahre nach dem Abschluss ist relativ einheitlich etwa ein Zehntel der Absolventinnen und Absolventen selbstständig tätig. In der Psychologie gibt es erwartungsgemäß deutlich mehr Selbstständige. 
Abbildung 3.4: Berufliche Stellung ein, fünf und zehn Jahre nach dem Studienabschluss, nach Abschlusskohorten (nur traditionelle Abschlüsse, in \%)

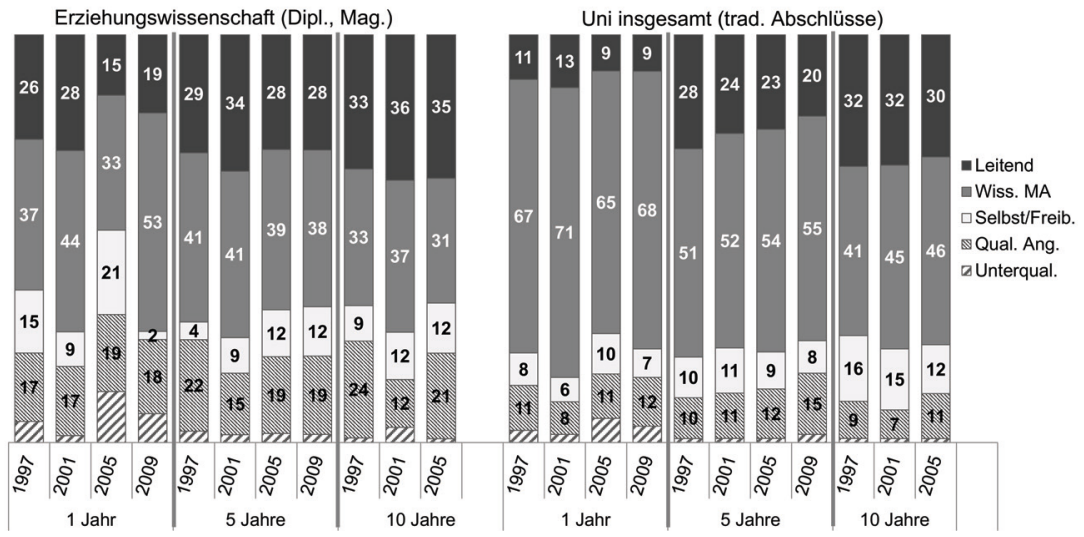

Quelle: DZHW-Absolventenpanel 1997, 2001, 2005 und 2009

In der Erziehungswissenschaft als Fach mit einem hohen Frauenanteil ist der Anteil der Erwerbstätigen in einer Vollzeitbeschäftigung deutlich niedriger als insgesamt für alle Hochschulabsolventinnen und -absolventen und in den Vergleichsfächern. Zehn Jahre nach dem Abschluss ist nur etwas mehr als die Hälfte vollzeiterwerbstätig (55 bis 60 \%). Ein seit der Kohorte 1997 von 16 auf $26 \%$ steigender Anteil ist in »langer Teilzeit « (21 bis 35 Stunden) erwerbstätig; der Anteil in »kurzer« Teilzeit (bis 20 Stunden) nimmt von 24 auf $17 \%$ ab. In der Psychologie sowie dem Sozialwesen ist der Anteil an Vollzeitbeschäftigten ebenfalls eher gering. Insgesamt sind etwa drei Viertel der erwerbstätigen Universitätsabsolventinnen und -absolventen zehn Jahre nach dem Studienabschluss vollzeitbeschäftigt.

\section{Wirtschaftszweige und öffentlicher Dienst}

Bis zum Ausgang des 19. Jahrhunderts war der Staatsdienst der dominierende Beschäftigungssektor für Hochschulabsolventinnen und -absolventen. Seitdem nimmt die Bedeutung der anderen Arbeitsmarktsegmente, insbesondere der Beschäftigung in der Privatwirtschaft, zu. Dabei gibt es jedoch große fachspezifische Differenzen. Immer noch spielt die Beschäftigung im öffentlichen Dienst aber eine zentrale Rolle für Absolventinnen und Absolventen eines Hochschulstudiums. So wird auch in den jüngeren Kohorten mittel und langfristig über alle Fächer hinweg etwa ein Drittel der Absolventinnen und Absolventen in 
verschiedenen Bereichen des öffentlichen Dienstes - einschließlich vergleichbarer Beschäftigungsverhältnisse - tätig (vgl. Autorengruppe Bildungsberichterstattung 2018: 167f.). In einzelnen Bereichen ist die Beschäftigung im öffentlichen Dienst weiterhin dominant, hier beim Sozialwesen und vor allem beim Lehramt. In der Erziehungswissenschaft spielt der öffentliche Dienst als Arbeitgeber ebenfalls eine größere Rolle (Abbildung 3.5). Bei den Kohorten 2001 und 2005 wurden hier bis zehn Jahre nach Studienabschluss etwa $60 \%$ der Absolventinnen und Absolventen erwerbstätig. Beim Jahrgang 1997 waren es sogar knapp $70 \%$. Fachkräfte mit einem erziehungswissenschaftlichen Abschluss bilden offenbar auch über das Lehramt hinaus einen Teilarbeitsmarkt, der stark von der Nachfrage des öffentlichen Dienstes nach akademisch qualifiziertem Personal geprägt wird.

Abbildung 3.5: Anteil im öffentlichen Dienst Beschäftigter zehn Jahre nach dem Studienabschluss, nach Abschlusskohorten und Fachrichtungen (nur traditionelle Abschlüsse, in \%)

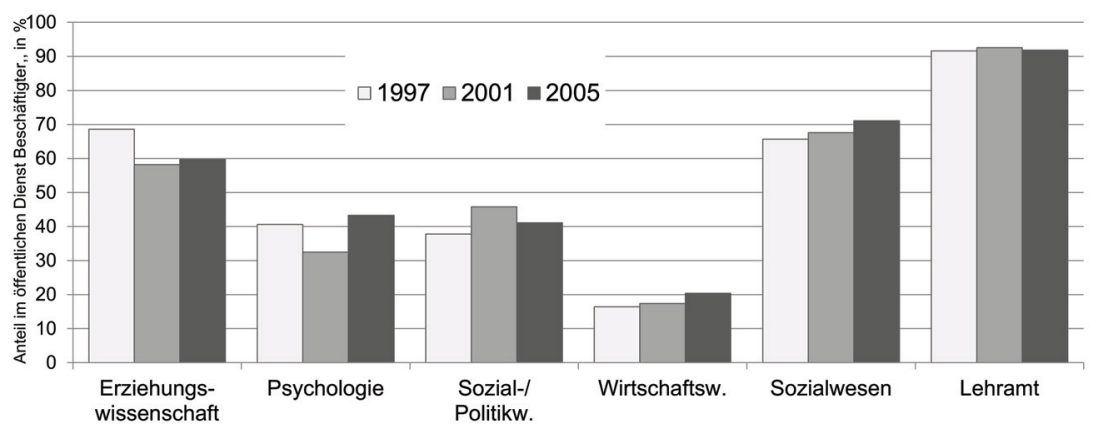

Quelle: DZHW-Absolventenpanel 1997, 2001, 2005

Die wichtigsten Wirtschaftszweige für die Erziehungswissenschaften sind die sozialen Dienste sowie Tätigkeiten im Bereich Bildung und Forschung (ohne Tabelle). Etwa ein Drittel ist zehn Jahre nach dem Abschluss in einer Einrichtung tätig, die zu den sozialen Diensten zählt. Im Vergleich der drei Kohorten 1997, 2001 und 2005 hat dieser Anteil zugenommen (von 27 über 31 auf $34 \%$ ). Ein gleichbleibender Anteil von etwa $8 \%$ gibt eine Beschäftigung in der öffentlichen Verwaltung an, während etwa jede/r siebte bei Verbänden oder Kirchen erwerbstätig ist. Ein weiteres Viertel wird im Bereich Bildung, Forschung und Kultur erwerbstätig, davon die meisten an Schulen (8 bis $10 \%$ ) und in Weiterbildungseinrichtungen (5 bis $9 \%$ ). Etwa $7 \%$ sind zehn Jahre nach dem Abschluss an Hochschulen und Forschungseinrichtungen tätig. In der zweiten Befragungswelle fünf Jahre nach Studienabschluss ist dieser Anteil aufgrund der laufenden Promotionen etwas höher. 


\section{Einkommen}

In dieser Ausgabe des Datenreports werden die Bruttostundengehälter für die Abschlusskohorten 1997, 2001 und 2005 jeweils fünf und zehn Jahre nach dem Studienabschluss miteinander verglichen (Abbildung 3.6); hinzu kommen die Angaben für die Kohorte 2009 zum Einkommen nach fünf Jahren. ${ }^{16}$ Dieser Indikator hat gegenüber dem Jahreseinkommen den Vorteil, dass auch das Einkommen der in der Erziehungswissenschaft hohen Zahl an Teilzeitbeschäftigten mit berücksichtigt werden kann. ${ }^{17}$ Im Fächervergleich liegt das Einkommen in der Erziehungswissenschaft in allen Kohorten jeweils am unteren Rand des Einkommensspektrums der universitären Vergleichsfächer (Abbildung 3.6). Die höchsten Einkommen werden mit einem wirtschaftswissenschaftlichen Abschluss erzielt; in der Psychologie wird das zweithöchste Einkommensniveau erreicht, das etwa dem Durchschnitt aller Universitätsabsolventinnen und -absolventen entspricht. Auch in den Sozial- und Politikwissenschaften wird ein etwas höheres Einkommen erzielt als in der Erziehungswissenschaft. Die Erziehungswissenschaft liegt im Bruttoeinkommen mit dem Lehramt Sekundarstufe I auf etwa gleichem Niveau, aber unter dem Lehramt Sekundarstufe II, wobei allerdings die Nettoeinkommen der Lehrerinnen und Lehrer aufgrund der hohen Verbeamtungsquote höher ausfallen dürften. Noch etwas geringer als in der Erziehungswissenschaft ist das Einkommen im Sozialwesen. Hier manifestiert sich die unterschiedliche Einstufung je nach Art des Hochschulabschlusses im öffentlichen Dienst.

Für Beschäftigte aus allen Vergleichsfächern zeigt sich erwartungsgemäß eine Erhöhung des Einkommens zwischen der zweiten und dritten Befragungswelle (Abbildung 3.6). Zehn Jahre nach dem Studienabschluss liegt das Einkommen pro Stunde mit einem Universitätsabschluss in allen drei Befragungskohorten, für die Daten bis zu zehn Jahre nach dem Abschluss vorliegen, etwa um ein Drittel höher als zum Zeitpunkt der zweiten Befragung, fünf Jahre nach dem Abschluss. Für die Erziehungswissenschaft ist im Zeitraum von fünf bis zehn Jahren nach Studienabschluss für die ersten beiden Kohorten 1997 und 2001 ein etwas geringerer Zuwachs von etwa $20 \%$ zu verzeichnen, während beim Jahrgang 2005 das Einkommen in diesem Zeitraum mit $40 \%$ überdurchschnittlich stark anstieg. Der Frage, welche Rolle mögliche Kompositionseffekte, unterschiedliche Anteile von Teilzeitbeschäftigten, die möglicherweise in schlechter vergüteten Positionen arbeiten, oder ökonomische Rahmenbedingungen (Finanzkrise, tarifvertragliche Entwicklungen wie die Ablösung des BAT durch den TVL bzw. TVöD im Jahr 2006) für die Kohortenunterschiede spielen, kann hier nicht nachgegangen werden.

16 Basis sind die Einkommen einschließlich fester Zulagen (etwa 13. Gehälter) sowie variabler Gehaltsbestandteile, die jedoch nur in wenigen Fachrichtungen eine größere Bedeutung haben, etwa in den Wirtschaftswissenschaften.

17 Es werden nur Teilzeitbeschäftigte mit mindestens 20 Wochenstunden und Vollzeiterwerbstätige berücksichtigt. 
Abbildung 3.6: Vergleich der Einkommen (Bruttostundenlohn in Euro) einschl. aller Zulagen ${ }^{1}$ fünf und zehn Jahre nach dem Studienabschluss, nach Abschlusskohorten und Fachrichtungen ${ }^{2}$

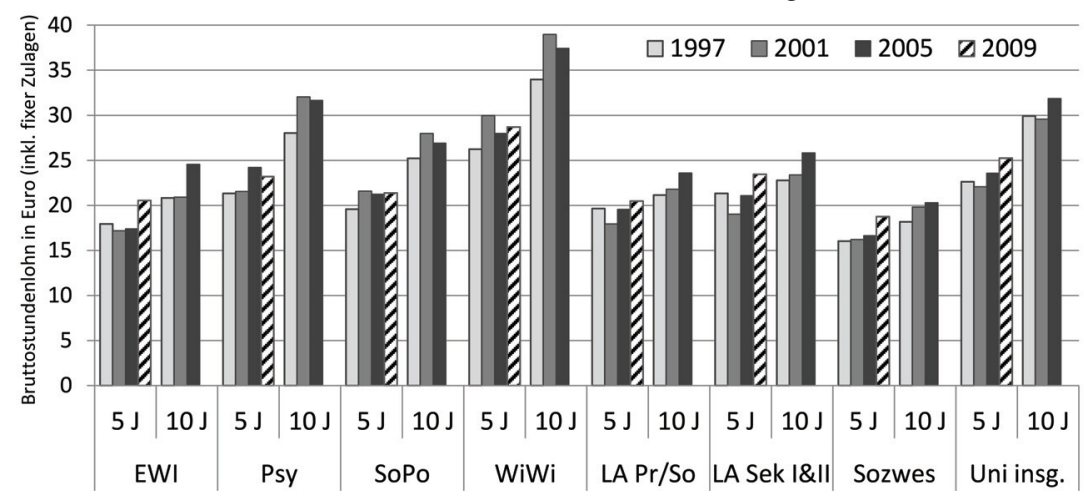

1 Werte für zweite Befragung 1997 (5 Jahre) ohne variable Zulagen

2 Nur Erwerbstätige mit mindestens 20 Stunden Wochenarbeitszeit, traditionelle Abschlüsse, in Euro, nominal

Quelle: DZHW-Absolventenpanel 1997, 2001, 2005, 2009

\section{Berufliche Zufriedenheit}

Beruflicher Erfolg drückt sich auch in der beruflichen Zufriedenheit aus, die neben der Bewertung monetärer Kriterien wie dem Einkommen auch die Beurteilung nicht-monetärer Aspekte wie Arbeitsinhalte, Beschäftigungssicherheit oder Arbeitszeitregelungen beinhaltet. Während im Datenreport 2016 über die Arbeitszufriedenheit nur für die Absolventenkohorte 2001, zehn Jahre nach dem Studienabschluss, berichtet wurde, soll hier die Betrachtung um den Vergleich mit den beiden Kohorten 1997 und 2005 erweitert werden. Die verschiedenen Items, mit denen die berufliche Zufriedenheit erfasst wurde, werden der Übersichtlichkeit wegen zu drei Zufriedenheitsdimensionen gebündelt (vgl. Briedis/Fabian 2009: 66): die Zufriedenheit mit den Arbeitsinhalten und den Arbeitsbedingungen ${ }^{18}$, die Zufriedenheit mit den Erträgen und den beruflichen Karriereperspektiven ${ }^{19}$ sowie die Zufriedenheit mit den zeitlichen Rahmenbedingungen der Arbeit ${ }^{20}$. In der Abbildung 3.7 sind die Mittelwerte einer

18 Hierzu gehören die Items Zufriedenheit mit Tätigkeitsinhalten, Arbeitsbedingungen, technische Ausstattung, Möglichkeit, eigene Ideen einzubringen sowie Arbeitsklima.

19 Items Einkommen, berufliche Position, Aufstiegsmöglichkeiten, Beschäftigungssicherheit sowie Qualifikationsangemessenheit.

20 Items Raum für Privatleben, Arbeitszeitorganisation, Umfang/Länge der Arbeitszeit, Familienfreundlichkeit. 
Fünferskala angegeben, die so gepolt wurde, dass höhere Werte eine größere Zufriedenheit anzeigen. Mit den ergänzend angegebenen Konfidenzintervallen lässt sich abschätzen, ob Unterschiede signifikant sind.

Bei der Bewertung der Zufriedenheit mit den Arbeitsbedingungen und den Arbeitsinhalten gibt es nur relativ geringe Unterschiede zwischen den Kohorten und Vergleichsfächern. Für die Universitätsabsolventinnen und -absolventen insgesamt lässt sich im Kohortenvergleich eine leichte Zunahme der Zufriedenheit erkennen, die aber nur zwischen 1997 und 2005 signifikant ausfällt. Diese Zunahme in der Zufriedenheit zeigt sich auch in den meisten der Vergleichsfächer, jedoch zumeist nicht in signifikantem Ausmaß. Die Erziehungswissenschaft liegt etwa auf dem durchschnittlichen Niveau, wobei sich die Werte zwischen den drei Kohorten nicht unterscheiden. Mit Mittelwerten von mindestens 4,0 ist die Zufriedenheit in der Psychologie mit den Arbeitsinhalten am größten und unterscheidet sich deutlich von der im Lehramt oder dem Sozialwesen.

Bei den Erträgen und Beschäftigungsperspektiven unterscheiden sich die Fachrichtungen, teilweise auch die Kohorten, deutlich stärker voneinander. Insgesamt ist die Zufriedenheit in dieser Dimension etwas geringer als mit den Arbeitsinhalten und -bedingungen. Bei den Erträgen und Perspektiven lässt sich im Vergleich der drei Kohorten ebenfalls eine deutliche Zunahme der Zufriedenheit feststellen, die sich auch in der Erziehungswissenschaft zeigt. Die Zunahme der Zufriedenheit zwischen den beiden Kohorten 1997 und 2005 zeigt sich in allen Vergleichsfächern. Am zufriedensten mit den Erträgen und Perspektiven sind die Lehrerinnen und Lehrer; sie unterscheiden sich hier deutlich von den Beurteilungen in den Erziehungs-, Sozial- und Politikwissenschaften sowie dem Sozialwesen. In die Zufriedenheit mit den Erträgen und Beschäftigungsperspektiven gehen verschiedene Teilaspekte ein, die in den Fachrichtungen unterschiedlich ausgeprägt sind. So beurteilen Lehrerinnen und Lehrer die Beschäftigungssicherheit als Beamte erwartungsgemäß sehr hoch und sind auch mit ihrem Einkommen vergleichsweise zufrieden, während sie mit den Aufstiegschancen weniger zufrieden sind. In den anderen Fachrichtungen, besonders ausgeprägt in der Erziehungswissenschaft, werden hingegen alle drei Teilaspekte eher selten als zufriedenstellend beurteilt (Abbildung 3.7).

Bei der Zufriedenheit mit den zeitlichen Rahmenbedingungen der Arbeit schließlich gibt es nur wenige Unterschiede zwischen den Fachrichtungen. Insgesamt wird auch diese Dimension vielfach etwas schlechter bewertet als die Arbeitsinhalte. Für die Universitätsabsolventinnen und -absolventen insgesamt sowie für die Lehrämter, die Wirtschaftswissenschaft und das Sozialwesen zeigt sich zwischen 1997 und 2005 eine Erhöhung der Zufriedenheit; in den übrigen Fächern, auch in der Erziehungswissenschaft, gibt es zwar auch diese Tendenz. Die Unterschiede sind aber nicht signifikant. 
Abbildung 3.7: Zufriedenheit mit Arbeitsinhalten und Arbeitsbedingungen, Erträgen und Perspektiven sowie Arbeitszeit und Vereinbarkeit $^{1}$ zehn Jahre nach dem Studienabschluss ${ }^{2}$ (Mittelwerte und Konfidenzintervalle)

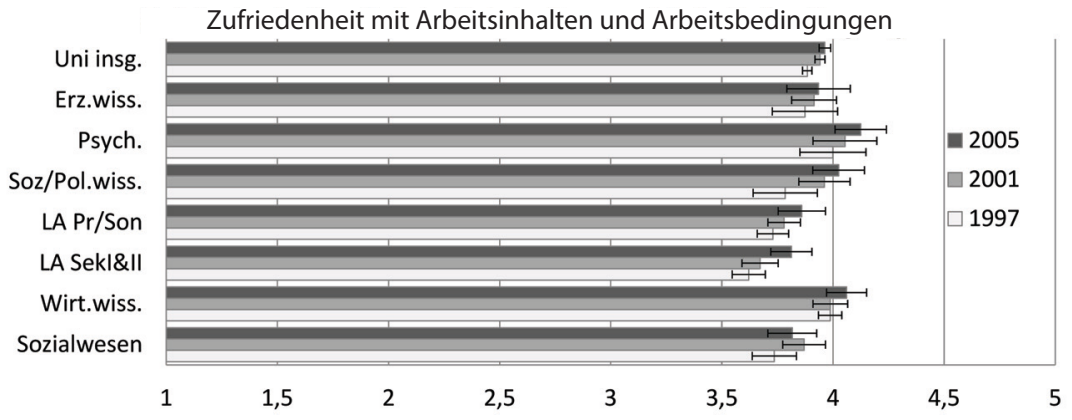

Zufriedenheit mit Erträgen und Perspektiven

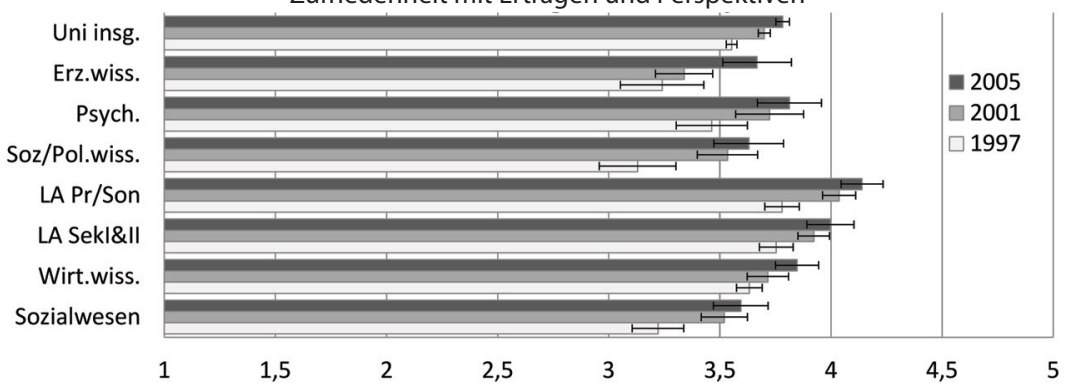

Zufriedenheit mit Arbeitszeit und Familienfreundlichkeit

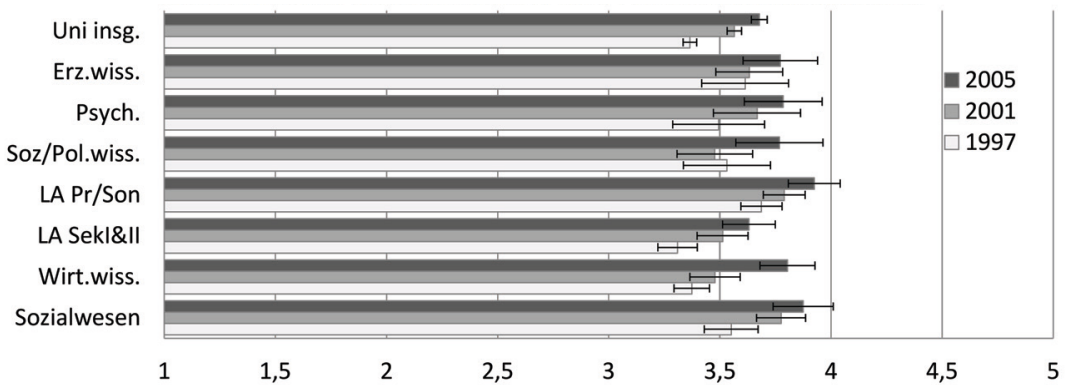

1 Mittelwerte einer Fünferskala von $1=$ sehr zufrieden bis $5=$ unzufrieden sowie Konfidenzintervalle auf dem $5 \%$-Niveau. Für die grafische Darstellung wurde die Skala umgedreht (5=sehr zufrieden), sodass höhere Werte größere Zufriedenheit anzeigen.

2 Absolventenkohorten 1997, 2001 und 2005.

Quelle: DZHW Absolventenpanel 1997, 2001 und 2005, eigene Berechnungen 


\subsection{Zusammenfassung der Ergebnisse im Kontext der bisherigen Datenreporte}

Werden die Ergebnisse dieses Datenreports zum beruflichen Verbleib der Absolventinnen und Absolventen erziehungswissenschaftlicher (Hauptfach-) Studiengänge im Zusammenhang mit den Datenreporten 2008, 2012 und 2016 betrachtet, dann zeigen sich in allen vier Berichten ähnliche Grundmuster der Übergänge in den Beruf und des beruflichen Verbleibs, wenngleich in unterschiedlichen Größenordnungen und Zeiträumen der beruflichen Einmündung und Konsolidierung. Im Unterschied zu den älteren Datenreporten, die auf den Absolventenkohorten 1997, 2001, 2005 und 2009 basieren, dominieren in dem aktuellen Bericht, der die Erstbefragung des Absolventenjahrgangs 2013 sowie zusätzlich einige Wiederholungsbefragungen für die älteren Kohorten einbezieht, die neuen Abschlüsse Bachelor und Master. Die Erziehungswissenschaft ist unter den an deutschen Hochschulen vorhandenen Studienfächern nicht nur eines der Fächer mit den höchsten Absolventenzahlen (siehe Abschnitt 3.1), sondern weist auch seit Einführung des Bachelor/Master-Studiensystems mit Ausnahme einzelner Jahre auf beiden Ebenen steigende Absolventenzahlen auf, auch wenn die Übergangsquote vom Bachelor in den Master niedriger ausfällt als in anderen Fächern. In Absolventenstudien, die Daten über den beruflichen Verbleib fünf oder zehn Jahre nach Hochschulabschluss bereitstellen, handelt es sich jedoch bei der Mehrzahl der Befragten noch um Absolventinnen und Absolventen mit den alten Abschlüssen.

In der öffentlichen Wahrnehmung gilt die Erziehungswissenschaft ähnlich wie andere geistes-, kultur- und sozialwissenschaftliche Fächer oft als ein Fach mit eher prekären Beschäftigungsperspektiven. Die Daten aus den Absolventenstudien zeigen aber, dass Berufseintritt und längerfristiger Verbleib deutlich besser ausfallen, als dem Fach nachgesagt wird. Danach ist die Erziehungswissenschaft zwar ein Fach, dessen Absolventinnen und Absolventen bei einigen Indikatoren häufigere Integrationsprobleme in den Arbeitsmarkt aufweisen, die sich jedoch bei keinem Indikator und keiner Kohorte zu einer Art Prekarisierung der Beschäftigungsverhältnisse verfestigen. In den Verläufen zwischen den Kohorten können aber Unterschiede bestehen: So fallen die Übergänge in die Erwerbstätigkeit, die Arbeitsmarktsituation und die Beschäftigungsbedingungen des Absolventenjahrgangs 2009 besser aus als bei den Jahrgängen 2005 und 1997. Verschiedene Kohorten können auf variierende Arbeitsmarktkonstellationen treffen und die berufliche Einmündung verläuft dann unterschiedlich. Die Kohorte 2013 weist ebenfalls keine gravierenden Übergangsprobleme auf, ist jedoch mit den älteren Jahrgängen kaum zu vergleichen, weil sich hier mit dem Bachelor und Master die Übergangssequenzen ausdifferenzieren.

Zwar wechselt in der Kohorte 2013 ein überdurchschnittlich hoher Anteil der Absolventinnen und Absolventen der Erziehungswissenschaft nach dem Bachelorabschluss in eine Erwerbstätigkeit. Aber ein Jahr nach dem 
Abschluss sind Arbeitslosigkeit und Jobben nicht häufiger als im Durchschnitt aller Absolventinnen und Absolventen eines Universitätsstudiums bzw. der Vergleichsfächer. Ähnliches gilt für die Übergänge nach dem Masterabschluss. Die durchschnittliche Suchphase nach dem Bachelorabschluss beträgt etwa drei bis vier Monate, nach dem Masterabschluss einen Monat länger. Dies entspricht den Werten für die früheren Jahrgänge. Die Kohorte 2013 weist beim Berufsstart keine besonderen Einmündungsprobleme in den Arbeitsmarkt auf, die sich grundlegend von den Vergleichsfächern unterscheiden. Auffällig sind zum einen eine deutlich niedrigere Übergangsquote der Bachelors in ein Masterstudium, nur die entsprechende Quote im Sozialwesen fällt noch niedriger aus; zum anderen bei den Mastern ein deutlich seltenerer Übergang in ein Promotionsstudium. Insgesamt gibt es aber wie in den früheren Datenreporten keine Evidenz für ausgesprochen krisenhafte Übergänge in Beschäftigung.

Für die älteren Kohorten - ein Vergleich mit der Kohorte 2013 ist auf der Bachelorebene wegen der anderen Übergangsstruktur und der noch nicht verfügbaren Folgebefragungen nicht möglich - lässt sich feststellen, dass die Einmündungsquote in reguläre Beschäftigung ein Jahr nach Hochschulabschluss zwischen 63 \% (für die Kohorte 2005) und 80 \% (2009) schwankt; nach drei Jahren wird in allen Kohorten ein Beschäftigungsniveau von mindestens 80 \% erreicht. Im Durchschnitt aller Fächer beträgt dieses ca. 85 \%, von den Vergleichsfächern schneiden nur die Wirtschaftswissenschaften besser ab. Hauptgrund für die niedrigere Beschäftigungsquote ist nicht Arbeitslosigkeit, sondern Elternzeit und Familienarbeit. Bei den Mastern des Jahrgangs 2013 liegt der Anteil regulärer Erwerbstätigkeit in der Erziehungswissenschaft mit Ausnahme der Wirtschaftswissenschaften und des Lehramts (Sek II) höher als in den Vergleichsfächern. Der wichtigste Grund für diesen Unterschied ist die schon erwähnte niedrigere Promotionsquote.

Die neben den Übergängen vom Studium in die Erwerbstätigkeit zweite Hauptfrage dieses Kapitels bezieht sich auf die Qualität der Beschäftigung. Hier haben sich die Beschäftigungsmerkmale zwischen den Kohorten 2005 und 2009 etwas verbessert, wenngleich sie in beiden Kohorten nicht einem Normalarbeitsverhältnis entsprechen. Nach einem Jahr ist nur ein geringer Anteil unbefristet beschäftigt (2005 29\%, $200935 \%$ ), ein hoher Anteil in Teilzeit tätig (2005 44 \%, $200962 \%)$; die Einkommen fallen unterdurchschnittlich, der wissenschaftliche Bezug der Tätigkeiten (z.B. Verbleib im Wissenschaftssystem) gering aus. Ein wichtiger Indikator für die Qualität der Beschäftigung ist die Adäquanz im Verhältnis von erworbener Qualifikation und ausgeübter Beschäftigung. Verglichen mit der Kohorte 2005, fällt die Adäquanz der Tätigkeiten bei der Kohorte 2009 zwar höher aus, erreicht aber in beiden Kohorten nur unterdurchschnittliche Werte. Nur etwa ein Drittel ist nach einem Jahr volladäquat beschäftigt, eine fast genauso große Gruppe dagegen inadäquat. Dieser Anteil fällt höher aus als in den anderen Vergleichsfächern. Auch bei der 
Kohorte 2013 zeichnet sich die erste Beschäftigung sowohl bei den Bachelors wie bei den Mastern häufiger als in den anderen Fächern durch eine vertikale Inadäquanz aus; so ist für mehr als $40 \%$ bei den Bachelors und etwa ein Drittel bei den Mastern der erreichte Abschluss für die (erste) Beschäftigung nicht zwingend erforderlich.

Wie in anderen Fächern gilt es aber auch in der Erziehungswissenschaft, den beruflichen Verbleib als einen längerfristigen Prozess zu betrachten, bei dem bis ins fünfte Jahr der Anteil regulärer Beschäftigung kontinuierlich anwächst und Arbeitslosigkeit und Inadäquanz abnehmen. Allerdings zeigen sich in der Erziehungswissenschaft die im Vergleich höheren Inadäquanzwerte auch zu allen späteren Befragungszeitpunkten. So ist auch zehn Jahre nach Abschluss der Anteil der inadäquat Beschäftigten in der Erziehungswissenschaft etwa doppelt so hoch wie im Durchschnitt. Zwar gelingt ihnen oft ein zügiger Berufseinstieg, aber um den Preis eines hohen Anteils inadäquater Beschäftigung. Bei fast allen Indikatoren schneiden sie schlechter ab als die Absolventinnen und Absolventen der Wirtschaftswissenschaft und Psychologie, wenn auch ähnlich, teilweise sogar günstiger als die der Sozialwissenschaften. Fünf bzw. zehn Jahre nach Studienabschluss zeichnet sich eine deutliche Verbesserung der beruflichen Situation ab, sodass ein schwieriger Start bis zu einem gewissen Maße ausgeglichen wird. Aber die Gesamtsituation bleibt immer noch etwas schwieriger als im Durchschnitt. Absolventinnen und Absolventen erziehungswissenschaftlicher Studiengänge weisen also eine insgesamt vergleichsweise ungünstige, wenn auch nicht prekäre Positionierung auf dem Arbeitsmarkt und im Beschäftigungssystem auf.

\section{Literatur}

Autorengruppe Bildungsberichterstattung (2016): Bildung in Deutschland. Ein indikatorengestützter Bericht mit einer Analyse zu Bildung und Migration. Bielefeld: wbv.

Autorengruppe Bildungsberichterstattung (2018): Bildung in Deutschland. Ein indikatorengestützter Bericht mit einer Analyse zu Wirkungen und Erträgen von Bildung. Bielefeld: wbv.

Fabian, Gregor/Briedis, Kolja (2009): Aufgestiegen und erfolgreich? Ergebnisse der dritten HIS-Absolventenbefragung des Jahrgangs 1997 zehn Jahre nach dem Examen. HIS Forum Hochschule 2/2009. Hannover: HIS.

Fabian, Gregor/Hillmann, Julika/Trennt, Fabian/Briedis, Kolja (2016): Hochschulabschlüsse nach Bologna. Werdegänge der Bachelor- und Masterabsolventen des Prüfungsjahrgangs 2013. DZHW Forum Hochschule 1/2016. Hannover: DZHW.

Fehse, Stefanie/Kerst, Christian (2007): Arbeiten unter Wert? Vertikal und horizontal inadäquate Beschäftigung von Hochschulabsolventen der Abschlussjahrgänge 1997 und 2001. In: Beiträge zur Hochschulforschung 29, 1, S. 72-98. 
Heublein, Ulrich/Ebert, Julia/Hutzsch, Christopher/Isleib, Sören/König, Richard/Richter, Johanna/Woisch. Andreas (2017): Zwischen Studienerwartungen und Studienwirklichkeit. Ursachen des Studienabbruchs, beruflicher Verbleib der Studienabbrecherinnen und Studienabbrecher und Entwicklung der Studienabbruchquote an deutschen Hochschulen. DZHW Forum Hochschule 1/2017. Hannover: DZHW.

Kerst, Christian/Wolter, Andrä/Züchner, Ivo (2016): Studienabschlüsse und Arbeitsmarkt. In: DGfE: Datenreport Erziehungswissenschaft 2016. Hrsg. v. Koller, H.-C./FaulstichWieland, H./Weishaupt, H./Züchner, I. Schriften der Deutschen Gesellschaft für Erziehungswissenschaft. Opladen u.a.: Verlag Barbara Budrich, S. 99-134.

Krüger, Hans-Hermann/Rauschenbach, Thomas/Fuchs, Kirsten/Grunert, Cathleen/Huber, Andreas/Kleifgen, Beate/Rostampour, Parviz/Seeling, Claudia/Züchner, Ivo (2003): Diplom-Pädagogen in Deutschland. Survey 2001. Weinheim/München: Juventa.

Rauschenbach, Thomas/Züchner, Ivo (2004): Studium und Arbeitsmarkt der Hauptfachstudierenden. In: DGfE: Datenreport Erziehungswissenschaft 2004. Hrsg. v. Tippelt, R./Rauschenbach, T./Weishaupt, H. Wiesbaden: VS Verlag für Sozialwissenschaften, S. 39-54.

Statistisches Bundesamt (2019): Prüfungen an Hochschulen 2018. Fachserie 11, Reihe 4.2. Wiesbaden.

Wolter, Andrä (2015): The Labour Market for Teachers in Germany - The Discrepancy between Need and Affordability. In: v. Buer, J./Kuhlee, D./Winch, C. (Hrsg.): Governance in der Lehrerausbildung: Analysen aus England und Deutschland. Wiesbaden: Springer VS, S. 191-208.

Wolter, Andrä/Koepernik, Claudia (2012): Studium und Beruf. In: Hans-Böckler-Stiftung (Hrsg.): Expertisen für Hochschule der Zukunft. Demokratische und soziale Hochschule. Bad Heilbrunn: Klinkhardt, S. 273-340. 



\section{Personal}

\section{Marius Gerecht, Heinz-Hermann Krüger, Markus Sauerwein \& Johanna Schultheiß}

Im vergangenen Datenreport Erziehungswissenschaft 2016 setzte sich der Trend zum Ausbau des Personals in der Erziehungswissenschaft über alle beteiligten Berufsgruppen fort. Sowohl die Professuren ${ }^{1}$, der Mittelbau als auch die Zahl wissenschaftlicher Hilfskräfte stiegen teilweise kräftig an, wobei die Berufsgruppe der Lehrkräfte für besondere Aufgaben einen besonders starken Aufwuchs erlebte. Da dieser Befund zum Lehrpersonal im vergangenen Berichtszeitraum nicht durch kurzfristige Änderungen im Lehrbedarf erklärt werden konnte (etwa sprunghafte Anstiege der Studierendenzahlen), wird es spannend sein zu sehen, ob sich diese Entwicklung weiter fortgesetzt hat. Im vergangenen Berichtszeitraum nahm auch die Anzahl der Professuren weiter zu, lag aber immer noch unter dem 1995 eingeleiteten Rückbau der Erziehungswissenschaft. Der vor zehn Jahren einsetzende (Wieder-)Aufbau und der damit auch verbundene forcierte Ausbau des Mittelbaus hat zu einer Annäherung an die Personalstrukturen anderer Fächer der Sozialwissenschaften wie etwa der Psychologie und Politikwissenschaften beigetragen, dennoch blieben die Spezifika der Erziehungswissenschaften, die sich beispielsweise durch einen höheren Lehrbedarf auszeichnet, weiterhin sichtbar. Die Frage, welche inhaltlichen Schwerpunkte in der Erziehungswissenschaft vom jüngsten Ausbau profitieren, wird zumindest auf professoraler Ebene im weiteren Verlauf dieses Berichts beantwortet.

Ein weiterer Schwerpunkt dieses Kapitels liegt in der Betrachtung der Entwicklung befristeter hauptberuflicher Arbeitsverhältnisse, die oftmals mit Unsicherheiten in der zukünftigen Lebensplanung verknüpft sind, besonders nach erfolgreicher Promotion. Die vorliegenden Daten können Aufschluss darüber geben, ob sich die unbefriedigende Situation mit einer Vielzahl befristeter Stellen im Mittelbau weiter manifestiert. Zwar haben die Absolventen gute Chancen eine Beschäftigung zu finden (vgl. Kapitel 3), jedoch meist ohne längerfristige Perspektive insbesondere im universitären Mittelbau. Des Weiteren wird ein Blick auf die professoralen Stellenausschreibungen sowie die voraussichtlich frei werden Professuren und damit der Bedarfe des professoralen Nachwuchses geworfen. ${ }^{2}$ Mit der Einführung der Juniorprofessuren sollte der

1 Gemeint sind hier und im Folgenden keine Personalstellen, sondern Professorinnen und Professoren als Personen. Professuren wird als geschlechtsneutrales Wort verwendet.

2 Bedenklich scheint zudem, dass zentrale erziehungswissenschaftliche Professuren an außeruniversitären Forschungsinstituten nicht nur durch andere Disziplinen übernommen werden, sondern diese teilweise auch neu in anderen Disziplinen wie beispielsweise der Pädagogischen Psychologie angesiedelt werden. 
professorale Nachwuchs eigentlich gestärkt und ein alternativer Karriereweg zur Professur eröffnet werden. Die im vergangenen Berichtszeitraum kritisch hinterfragte Diskrepanz zwischen dem schleichenden Ausbau der Juniorprofessuren und dem erwarteten Bedarf des professoralen Nachwuchses wird auch in diesem Berichtszeitraum wieder thematisiert werden müssen.

Die vorliegende Beschreibung der Personalstruktur der Erziehungswissenschaft schließt an die Kapitel zum Personal der bisherigen Datenreporte an (Krüger/Weishaupt 2000; Krüger/Schmidt/Siebholz/Weishaupt 2004; Krüger/ Schnoor/Weishaupt 2008; Krüger/Kücker/Weishaupt 2012; Gerecht/Krüger/ Post/Weishaupt 2016). Gegenstand des ersten Abschnitts (4.1) ist die strukturelle Entwicklung der Erziehungswissenschaften im Bereich der Professuren (Abschnitt 4.1.1) und des Mittelbaus (Abschnitt 4.1.2) seit 2015. Darüber hinaus werden in Abschnitt 4.1.3 die Entwicklungen im Kontext benachbarter sozialwissenschaftlicher Fächer verglichen. Der zweite Abschnitt (4.2) dieses Kapitels beschreibt die Entwicklungen der Stellenausschreibungen erziehungswissenschaftlicher Professuren seit den 1990er Jahren und im Speziellen seit 2015 unter Einbezug der Fachhochschulen und einer Beschreibung der Entwicklung in den alten und neuen Bundesländern. Dazu wird ein Gesamtüberblick über die ausgeschriebenen erziehungswissenschaftlichen Professuren über die vergangenen 28 Jahre herangezogen (vgl. Abschnitt 4.2.1). Die Differenzierung nach Subdisziplinen der Erziehungswissenschaften erlaubt darüber hinaus eine präzisere Beschreibung der Entwicklungen in den Hochschulen und auch Fachhochschulen (vgl. Abschnitt 4.2.2). In Abschnitt 4.2.3 erfolgen dann eine Bilanzierung der ausgeschriebenen Professuren und eine Beschreibung der Chancen für den wissenschaftlichen Nachwuchs für die nächste Dekade. Den Abschluss des Kapitels bildet die Analyse der Geschlechterverhältnisse (Abschnitt 4.3) im Kontext der Personalstruktur, die im letzten Datenreport noch im Rahmen eines eigenen Kapitels diskutiert wurden. Dabei wird der Anteil der durch Frauen besetzten Professuren über ausgewählte Disziplinen hinweg in den Blick genommen wie auch die Entwicklung der Geschlechterverhältnisse für die Berufsgruppen im Mittelbau. Dazu werden Daten von 1995 bis 2018 herangezogen. Den Abschluss (Abschnitt 4.4) bildet ein Fazit über die in diesem Kapitel referierten Ergebnisse.

\subsection{Erziehungswissenschaftliches Personal im Innen- und Außenvergleich}

Der Innenvergleich der personellen Strukturen unterscheidet hauptsächlich die Entwicklungen zwischen ausgewählten Fachgebieten der Erziehungswissenschaften. Die Auswahl und die hier verwendete Terminologie folgen dabei der Hochschulstatistik, in der die Sonderpädagogik und die Sozialpädagogik 
seit 1992 als eigene Fächer geführt werden. ${ }^{3}$ Das in der Lehre und Forschung tätige Personal im Sozialwesen wird in diesem Kontext der Sozialpädagogik zugerechnet. Die Auswertungen beziehen sich nur auf wissenschaftliche Hochschulen (Universitäten, Pädagogische Hochschulen, Theologische Hochschulen und Kunsthochschulen). Weithin ausgeblendet bleibt in diesem Abschnitt das Personal der Fachhochschulen (Universities of Applied Sciences), da nahezu 95 \% des wissenschaftlichen Personals an wissenschaftlichen Hochschulen beschäftigt sind. Die folgenden Auswertungen beziehen sich auf erfasste Personen, nicht auf Stellenpläne oder (un-)besetzte Stellen. Die Datengrundlage dafür bildet die Hochschul-Personalstatistik des Statistischen Bundesamts seit Beginn der 1990er Jahre bis 2018. Nicht alle hier aufgeführten Daten wurden in dieser Form auch veröffentlicht. In diesen Fällen handelt es sich um Sonderauswertungen des Statistischen Bundesamts, die für den Datenreport angefordert und speziell aufbereitet wurden. ${ }^{4}$ Diese Sonderauswertungen sind in den Tabellen kenntlich gemacht.

Der Innenvergleich des erziehungswissenschaftlichen Personals gibt Aufschluss über die binnenspezifischen Entwicklungen innerhalb der Erziehungswissenschaften und den nach Datenlage differenzierbaren Fachgebieten Sonderpädagogik und Sozialpädagogik. Dazu zählen beispielsweise Verhältnisse von befristeten Stellen zu Dauerstellen oder die Relation von hauptberuflichen zu nebenberuflichen Beschäftigungsverhältnissen.

Zentrale Fragen dieses Kapitels sind: Setzen die sich im vergangenen Berichtszeitraum identifizierten Trends zu befristeten Beschäftigungen fort? Wie gestaltet sich die Lage von Vollzeitstellen im Mittelbau als Perspektive für Personal nach erfolgreicher Promotion jenseits der Professur? Sind die vorliegenden Zahlen als Zeichen wachsender Forschungsstärke in der Erziehungswissenschaft zu interpretieren? Was bedeuten diese Entwicklungen für die beruflichen Perspektiven des im Hochschulbereich ausgebildeten und arbeitenden Personals? Um diesen Fragen nachzugehen, werden alle Berufsgruppen der Erziehungswissenschaften im Hochschulbereich in den Blick genommen, dazu zählen die Professuren (siehe Abschnitt 4.1.1), der Mittelbau sowie analog zum letzten Datenreport 2016 auch die Gruppe der wissenschaftlichen Hilfskräfte (siehe Abschnitt 4.1.2). Darüber hinaus werden benachbarte Disziplinen wie die Psychologie, die Politikwissenschaften, die Sozialwissenschaften und die Wirtschaftswissenschaften für einen Fächervergleich herangezogen, um die identifizierten Tendenzen besser einordnen zu können. Die damit verbundene Kontextualisierung der Binnenverhältnisse erlaubt den Vergleich der stattgefunden Entwicklungen seit Mitte der 1990er Jahre (siehe Abschnitt 4.1.3).

3 Mit dieser Aufteilung werden keine Aussagen über disziplinäre Zugehörigkeiten getroffen.

4 Gedankt sei an dieser Stelle Brigitte Damm und Thomas Weise vom Statistischen Bundesamt für ihre stets kompetente und stets hilfreiche Unterstützung. 


\subsubsection{Professuren}

Ein Anstieg der Professuren in den Erziehungswissenschaften ist erfreulicherweise weiterhin zu verzeichnen, sodass nun beinahe wieder das Ausgangsniveau des Jahres 1995 erreicht ist (siehe Tabelle 4.1). Der Rückgang gegenüber dem Referenzjahr 1995 liegt nun bei 4 \% gegenüber 7 \% im vergangenen Berichtszeitraum. Dagegen haben sich die Stellen für wissenschaftliche Mitarbeiter und für Lehrkräfte für besondere Aufgaben (LfbA) seit 1995 mehr als verdoppelt. Neben dem Aufwuchs dieser hauptberuflichen Stellen ist auch beim nebenberuflichen Personal ein Anstieg um mehr als $40 \%$ bei den Stellenvolumina (absolut +1.094 Stellen) zu verzeichnen.

Tabelle 4.1: Hauptberufliches und nebenberufliches wissenschaftliches Personal in den Erziehungswissenschaften (inkl. Sonder- und Sozialpädagogik) an wissenschaftlichen Hochschulen 1995, 2000, 2005, 2010 und 2014-2018

\begin{tabular}{|c|c|c|c|c|c|c|}
\hline Jahr & insgesamt & Professuren & $\begin{array}{l}\text { Assistenten, } \\
\text { Dozenten }\end{array}$ & $\begin{array}{l}\text { wissen- } \\
\text { schaftliche } \\
\text { Mitarbeiter }\end{array}$ & $\begin{array}{l}\text { Lehrkräfte } \\
\text { für besonde- } \\
\text { re Aufgaben }\end{array}$ & $\begin{array}{l}\text { neben- } \\
\text { berufliches } \\
\text { Personal }\end{array}$ \\
\hline 1995 & 3.271 & 1.091 & 320 & 1.573 & 287 & 2.642 \\
\hline 2000 & 3.186 & 979 & 277 & 1.597 & 333 & 2.538 \\
\hline 2005 & 3.162 & 861 & 190 & 1.782 & 329 & 2.672 \\
\hline 2010 & 4.422 & 934 & 50 & 2.920 & 518 & 3.345 \\
\hline 2014 & 5.220 & 1.011 & 44 & 3.434 & 731 & 3.829 \\
\hline 2015 & 5.377 & 1.021 & 40 & 3.526 & 790 & 3.971 \\
\hline 2016 & 5.447 & 1.038 & 38 & 3.644 & 727 & 3.739 \\
\hline 2017 & 5.757 & 1.043 & 41 & 3.893 & 780 & 3.610 \\
\hline 2018 & 6.065 & 1.045 & 52 & 4.189 & 779 & 3.736 \\
\hline
\end{tabular}

Quelle: Statistisches Bundesamt, Sonderauswertungen der Hochschulpersonalstatistik

Differenziertere Einblicke in die Zuwächse hinsichtlich der Besoldungsgruppe und der Verteilung der Professuren in den einzelnen Studienbereichen Erziehungswissenschaft, Sonderpädagogik und Sozialpädagogik erlaubt Tabelle 4.2. Über alle Studienbereiche hinweg zeigt sich ein moderater Anstieg der Professuren um insgesamt 34 Stellen gegenüber dem letzten Berichtszeitraum. Bei den C4/W3-Professuren sind die Zuwächse am stärksten (+32 Stellen), während die C3/W2-Professuren nur geringe Zuwächse verzeichnen (+6 Stellen) und die C2-Professuren auf Dauer über alle Bereiche hinweg insgesamt um 6 Stellen schrumpfen. Die Anzahl der C2/W1-Professuren auf Zeit steigt marginal um 3 Stellen an. Damit setzt sich der Trend des Rückbaus der C2-Professuren auf Dauer hin zum Ausbau von C2/W1-Professuren auf Zeit seit 1995 weiter fort. Erhöht hat sich in diesem Kontext der Anteil der Juniorprofessuren von $61 \%$ auf $76 \%$ gegenüber dem Berichtsjahr 
2014, Zugewinne konnte dabei jedoch nur die Erziehungswissenschaft verzeichnen (+20 Stellen), während die Stellenvolumina der Juniorprofessuren in der Sonderpädagogik sogar um eine Stelle abnahm und in der Sozialpädagogik nur um eine Stelle zunahm. In der Erziehungswissenschaft umfassen Juniorprofessuren knapp $10 \%$ aller »Professuren «, in der Sonderpädagogik sind es $7 \%$ und in der Sozialpädagogik knapp $5 \%$. Zudem ist in den Bereichen der Sonderpädagogik und Sozialpädagogik eher ein Trend zur Stagnation denn zur Expansion in diesem Verhältnis zu verzeichnen. Vom Wiederausbau der professoralen Stellen seit 2010 profitiert die Sonderpädagogik am geringsten, war aber im gesamten Betrachtungsraum seit 1995 auch weniger vom Rückbau der Erziehungswissenschaften betroffen. Eine Expansion im universitären Umfeld erfährt die Sozialpädagogik durchgehend seit 1995. Die Erziehungswissenschaft als Fach war am stärksten vom Rückbau von 1995 bis 2010 betroffen, konnte in der Nachfolgezeit aber auch die stärksten Zuwächse verzeichnen.

Es zeigt sich, dass sich die Trends zum Anstieg der Professuren und auch zur Ausbildung des professoralen Nachwuchses über die Vergabe von Juniorprofessuren weiter fortsetzen. Fraglich bleibt jedoch, ob der spärliche Ausbau der Juniorprofessuren (+20 Stellen) in Geschwindigkeit und Umfang ausreicht, um eine nachhaltige Absicherung des Nachwuchses zu gewährleisten. Gemessen am geschätzten Bedarf, der bereits im vergangenen Datenreport problematisiert wurde, sind in der nächsten Dekade annähernd 300 neue Professuren über alle Studienbereiche der Erziehungswissenschaften hinweg neu zu besetzen (vgl. Abschnitt 4.2.3). Hoffnung macht in diesem Kontext der Aufwuchs des hauptberuflichen wissenschaftlichen Personals (vgl. Abschnitt 4.1.2), was die Rekrutierung der Juniorprofessuren erleichtern sollte, zugleich aber aufgrund des eher schleichend wachsenden Stellenangebots auch zu größerer Konkurrenz um eben diese Stellen führen könnte. Soll die Juniorprofessur ein wirksames Instrument zur Nachwuchsförderung für erziehungswissenschaftliche Professuren werden, muss die Quantität der Stellen und Stellenangebote deutlich erhöht sowie die Möglichkeiten zur Entfristung z.B. durch Tenure-Track-Professuren (weiter) vorangetrieben werden. Eine Alternative ist die Besetzung der Professuren aus dem Reservoir des wissenschaftlichen Mittelbaus ohne den Alternativweg Juniorprofessur und auch immer häufiger ohne den Weg via Habilitation (vgl. Kapitel 6). Darüber hinaus sorgt der Aufstieg der empirischen Bildungsforschung (vgl. Abschnitt 4.2.2) innerhalb der Erziehungswissenschaften für zusätzliche Konkurrenz, da diese Professuren auch für den Nachwuchs aus Nachbardisziplinen wie etwa der Psychologie, die sehr starken Wert auf die quantitative methodische Ausbildung des wissenschaftlichen Nachwuchses legt, attraktiv sind. Gleichzeitig können die Erziehungswissenschaften aber auch von Impulsen aus benachbarten Disziplinen profitieren. 
Tabelle 4.2: Professuren 1995, 2000, 2005, 2010 und 2014-2018 nach der Bewertung der Stelle (wissenschaftliche Hochschulen)

\begin{tabular}{|c|c|c|c|c|c|}
\hline \multirow[b]{2}{*}{ Jahr } & \multirow[b]{2}{*}{ insgesamt } & \multicolumn{4}{|c|}{ darunter ... und entsprechende Besoldungsgruppen } \\
\hline & & C4, W3 & $\mathrm{C} 3, \mathrm{~W} 2$ & C2 auf Dauer & C2, W1 auf Zeit $^{1}$ \\
\hline \multicolumn{6}{|c|}{ Erziehungswissenschaft } \\
\hline 1995 & 860 & 406 & 301 & 132 & 19 \\
\hline 2000 & 743 & 353 & 299 & 89 & 2 \\
\hline 2005 & 624 & 313 & 254 & 30 & 27 \\
\hline 2010 & 698 & 345 & 267 & 12 & $64(33)$ \\
\hline 2014 & 770 & 386 & 268 & 16 & $90(57)$ \\
\hline 2015 & 775 & 387 & 263 & 22 & $92(62)$ \\
\hline 2016 & 786 & 400 & 271 & 22 & $86(66)$ \\
\hline 2017 & 790 & 404 & 258 & 19 & $100(77)$ \\
\hline 2018 & 794 & 405 & 270 & 11 & $100(77)$ \\
\hline \multicolumn{6}{|c|}{ Sonderpädagogik } \\
\hline 1995 & 165 & 81 & 59 & 17 & 6 \\
\hline 2000 & 164 & 80 & 72 & 12 & 0 \\
\hline 2005 & 154 & 75 & 67 & 5 & 7 \\
\hline 2010 & 162 & 85 & 57 & 2 & $15(8)$ \\
\hline 2014 & 153 & 81 & 55 & 0 & $15(12)$ \\
\hline 2015 & 156 & 81 & 60 & 0 & $11(10)$ \\
\hline 2016 & 159 & 89 & 55 & 0 & $10(9)$ \\
\hline 2017 & 158 & 94 & 53 & 0 & $10(8)$ \\
\hline 2018 & 158 & 89 & 55 & 0 & $13(11)$ \\
\hline \multicolumn{6}{|c|}{ Sozialpädagogik } \\
\hline 1995 & 66 & 13 & 33 & 18 & 1 \\
\hline 2000 & 72 & 15 & 41 & 16 & 0 \\
\hline 2005 & 83 & 13 & 50 & 19 & 1 \\
\hline 2010 & 74 & 28 & 37 & 3 & $6(2)$ \\
\hline 2014 & 88 & 19 & 43 & 8 & $15(4)$ \\
\hline 2015 & 90 & 23 & 47 & 6 & $13(4)$ \\
\hline 2016 & 93 & 23 & 49 & 10 & $8(3)$ \\
\hline 2017 & 95 & 24 & 48 & 10 & $10(4)$ \\
\hline 2018 & 93 & 24 & 47 & 7 & $10(5)$ \\
\hline \multicolumn{6}{|c|}{ Erziehungswissenschaften insgesamt } \\
\hline 1995 & 1.091 & 500 & 393 & 167 & 26 \\
\hline 2000 & 979 & 448 & 412 & 117 & 2 \\
\hline 2005 & 861 & 401 & 371 & 54 & 35 \\
\hline 2010 & 934 & 458 & 361 & 17 & $85(43)$ \\
\hline 2014 & 1.011 & 486 & 366 & 24 & $120(73)$ \\
\hline 2015 & 1.021 & 491 & 370 & 28 & $116(76)$ \\
\hline 2016 & 1.038 & 512 & 375 & 32 & $104(78)$ \\
\hline 2017 & 1.043 & 522 & 359 & 29 & $120(89)$ \\
\hline 2018 & 1.045 & 518 & 372 & 18 & $123(93)$ \\
\hline 1995-2014 & -80 & -14 & -27 & -143 & 94 \\
\hline 1995-2018 & -46 & 18 & -21 & -149 & 97 \\
\hline
\end{tabular}

1 in Klammern: darunter Juniorprofessuren

Quelle: Statistisches Bundesamt, Sonderauswertungen der Hochschulpersonalstatistik 


\subsubsection{Mittelbau}

Im Abschnitt zum universitären Mittelbau werden Stellen im Rahmen der Qualifikationsphasen und der Post-Doktorandenphase jenseits der Professur bzw. auf dem Weg dorthin genauer betrachtet. Dazu zählen Dozenten und Assistenten sowie wissenschaftliche Mitarbeiter und Lehrkräfte für besondere Aufgaben. Im vergangenen Berichtszeitraum konnte ein fortschreitender Ausbau des Personalbestands im universitären Mittelbau verzeichnet werden. Dieser Trend hält an (vgl. Tabelle 4.3), auch wenn dieser Ausbau nun asymmetrisch vonstattengeht. Insgesamt beträgt die Zunahme der Stellen im Mittelbau 811 neue Stellen gegenüber dem Berichtsjahr 2014. Besonders stark ist der Zuwachs bei den wissenschaftlichen Mitarbeitern mit 755 Stellen seit 2014. Zieht man den Zuwachs gegenüber dem Berichtsjahr 2010 in Betracht, sind in den vergangen acht Jahren gar 1.269 neue Stellen für wissenschaftliche Mitarbeiter entstanden, was einer Aufstockung dieser Personalgruppe um ein Drittel gleich kommt. Die Zahl der Dozenten und Assistenten stagniert auf weiterhin sehr niedrigem Niveau und verzeichnet erstmals wieder geringe Zuwächse (+8 Stellen). Moderate Zuwächse gab es bei den Lehrkräften für besondere Aufgaben mit einem Plus von 48 Stellen. Der Anstieg der Lehrkräfte für besondere Aufgaben hat sich damit deutlich verlangsamt (der Zuwachs von 2010 zu 2014 betrug immerhin noch 213 Stellen). Damit zeichnet sich eine Asymmetrie gegenüber der Zunahme des hauptberuflichen wissenschaftlichen Personals und dem hauptsächlich für Lehrtätigkeiten bestimmten Personal ab. Es stellt sich die Frage, wie die wachsende Nachfrage nach universitärer Lehre basierend auf weiter steigenden Studierendenzahlen durch diese geringen Zuwachszahlen bedient werden kann, die auch nicht durch die moderate Zunahme der Juniorprofessuren aufgefangen wird (vgl. Abschnitt 4.1.1).

Wie bereits eingangs erwähnt, kann eine deutliche Zunahme von Stellen des hauptberuflichen wissenschaftlichen Personals (+29 \%) konstatiert werden. Dabei sind $40 \%$ der Angestellten auf Vollzeitstellen angesiedelt, was einer Zunahme dieses Anteils gegenüber dem Berichtsjahr 2014 um 3 \% entspricht. Jedoch entfallen die beiden größten Anteile zusätzlicher Stellen auf befristete Vollzeitstellen (+350 Stellen) und auf befristete Teilzeitstellen (+327 Stellen). Nur jede zehnte neue Stelle ist für diese Berufsgruppe auf Dauer ausgelegt. Der Anteil von Vollzeitstellen auf Dauer bei den wissenschaftlichen Mitarbeitern sinkt von $27 \%$ im Berichtsjahr 2014 auf $24 \%$ in 2018. Damit hat nur ein Viertel des wissenschaftlichen Personals eine unbefristete Vollzeitstelle inne. Auch bei den Teilzeitstellen sind unbefristete Beschäftigungsverhältnisse eher die Ausnahme als die Regel. Weiterhin verharrt der Anteil der unbefristeten Beschäftigungsverhältnisse in Teilzeit unter der Fünf-Prozent-Marke, was jedoch auch der Situation der Ausbildung des wissenschaftlichen Personals geschuldet ist, das zumeist in befris- 
teten Teilzeitverhältnissen beschäftigt ist. In diesem Kontext lassen sich diese Zahlen auch als Forschungsstärke interpretieren, sofern davon ausgegangen wird, dass ein Großteil dieser Stellen dem wissenschaftlichen Nachwuchs in der Qualifikationsphase zuzurechnen ist. Wird jedoch keine Teilzeitbeschäftigung oder (Weiter-)Qualifikation angestrebt, sind diese Stellen schwerlich dazu geeignet, eine befriedigende materielle Ausstattung der Arbeitnehmenden - insbesondere in einer Metropolregion - zu gewährleisten.

Tabelle 4.3: Struktur des hauptberuflichen Mittelbaus in der Erziehungswissenschaft (einschließlich Sonder- und Sozialpädagogik) 2010, 2014 und 2018 an wissenschaftlichen Hochschulen

\begin{tabular}{|c|c|c|c|c|c|c|c|}
\hline \multirow[b]{2}{*}{ Dienstbezeichnung } & \multicolumn{2}{|c|}{2010} & \multicolumn{2}{|c|}{2014} & \multicolumn{2}{|c|}{2018} & \multirow{2}{*}{$\begin{array}{c}\text { Veränderung } \\
2014 \text { bis } 2018 \\
\text { abs. }\end{array}$} \\
\hline & abs. & $\%$ & abs. & $\%$ & abs. & $\%$ & \\
\hline \multicolumn{8}{|c|}{ Dozenten und Assistenten } \\
\hline Vollzeitbeschäftigte & (33) & $(0,9)$ & (34) & $(0,8)$ & $(40)$ & $(0,8)$ & $(+6)$ \\
\hline Vollzeit auf Dauer & 14 & 0,4 & 8 & 0,2 & 8 & 0,2 & 0 \\
\hline Vollzeit befristet & 19 & 0,5 & 26 & 0,6 & 32 & 0,6 & +6 \\
\hline Teilzeitbeschäftigte & 17 & 0,5 & $(10)$ & $(0,2)$ & 12 & $(0,2)$ & $(+2)$ \\
\hline Teilzeit auf Dauer & - & - & 7 & 0,2 & 6 & 0,1 & -1 \\
\hline Teilzeit befristet & - & - & 3 & 0,1 & 6 & 0,1 & +3 \\
\hline \multicolumn{8}{|c|}{ wissenschaftliche Mitarbeiter } \\
\hline Vollzeitbeschäftigte & $(1.164)$ & $(33,4)$ & $(1.281)$ & $(30,4)$ & $(1.680)$ & $(33,5)$ & $(+399)$ \\
\hline Vollzeit auf Dauer & 364 & 10,4 & 350 & 8,3 & 399 & 7,9 & +49 \\
\hline Vollzeit befristet & 800 & 22,9 & 931 & 22,1 & 1.281 & 25,5 & +350 \\
\hline Teilzeitbeschäftigte & 1.756 & 50,3 & $(2.153)$ & $(51,2)$ & $(2.509)$ & $(50,0)$ & $(+356)$ \\
\hline Teilzeit auf Dauer & - & - & 82 & 1,9 & 111 & 2,2 & +29 \\
\hline Teilzeit befristet & - & - & 2071 & 49,2 & 2.398 & 47,8 & +327 \\
\hline \multicolumn{8}{|c|}{ Lehrkräfte für besondere Aufgaben } \\
\hline Vollzeitbeschäftigte & $(260)$ & $(7,5)$ & $(344)$ & $(8,2)$ & $(374)$ & $(7,5)$ & $(+30)$ \\
\hline Vollzeit auf Dauer & 127 & 3,6 & 136 & 3,2 & 166 & 3,3 & +30 \\
\hline Vollzeit befristet & 133 & 3,8 & 208 & 4,9 & 208 & 4,1 & 0 \\
\hline Teilzeitbeschäftigte & 258 & 7,4 & $(387)$ & $(9,2)$ & $(405)$ & $(8,1)$ & $(+18)$ \\
\hline Teilzeit auf Dauer & - & - & 45 & 1,1 & 80 & 1,6 & +35 \\
\hline Teilzeit befristet & - & - & 342 & 8,1 & 325 & 6,5 & -17 \\
\hline insgesamt & 3.488 & 100 & 4.209 & 100 & 5.020 & 100 & +811 \\
\hline
\end{tabular}

Quelle: Statistisches Bundesamt, Sonderauswertungen der Hochschulpersonalstatistik, eigene Berechnungen

Bei den Lehrkräften für besondere Aufgaben verfehlen die Anteile von Vollzeit- und Teilzeitbeschäftigungen nur knapp die Parität. Dabei überwiegen die Teilzeitbeschäftigungsverhältnisse mit $52 \%$. Die Vollzeitstellen für diese 
Berufsgruppe sind zu $44 \%$ auf Dauer ausgelegt, damit bieten über die Hälfte dieser Stellen keine Langzeitperspektive für die Beschäftigten. Bei den Teilzeitstellen sind $80 \%$ des dort ausgewiesenen Stellenvolumens befristet. Das bedeutet, dass eine Mehrzahl des vornehmlich für die Lehre tätigen hauptberuflichen Personals Befristungen ausgesetzt ist, wobei befristete Teilzeitstellen annähernd $42 \%$ des gesamten Stellenvolumens für Lehrkräfte mit besonderen Aufgaben ausmachen. Die geringe Zunahme von Dauerstellen auf Vollzeit und der hohe Anteil an befristeten Arbeitsverhältnissen auf Teilzeit können kaum dazu beitragen, den Ausbau der Lehre - bei steigenden Studierendenzahlen - nachhaltig abzusichern. Zudem sind diese Stellen eher niedrig vergütet, bei einem gleichzeitig hohen Arbeitsaufwand, der wenig Zeit für die weitere Qualifizierung erlaubt und damit wenige akademische Aufstiegschancen bietet. Darüber hinaus hat diese Berufsgruppe mit teils sehr kurzen Befristungen zu kämpfen, die einerseits die persönliche Unsicherheit der Betroffenen steigern und andererseits häufige Personalwechsel forcieren, was zusätzlich den Verlust von Know-how zur Folge hat und auch für Studierende bedeutet, sich auf Diskontinuitäten bei Lehre (und Prüfungen) sowie überfüllte Seminare einzustellen. Soll die Qualität der universitären Hochschullehre nachhaltig abgesichert werden, müssen die Zahl der befristeten Anstellungen weiter verringert sowie die Arbeitsbedingungen verbessert werden. Ebenso sollten auch diese Stellen Optionen zur wissenschaftlichen Weiterqualifikation beinhalten (mit der Option den Lehrumfang zu reduzieren), um die Attraktivität dieser Stellen zu erhöhen und damit auch zukünftig adäquates Personal akquirieren zu können.

Über alle Berufsgruppen des Mittelbaus hinweg sind nur $15 \%$ aller Stellen als Dauerstellen ausgewiesen, davon ist jede vierte Stelle eine Teilzeitstelle. Betrachtet man die Zuwächse über alle Berufsgruppen hinweg, bietet nicht mal jede fünfte neue Stelle eine längerfristige Perspektive. Zwar profitieren auch die Erziehungswissenschaften vom Nimbus des wissenschaftlichen Arbeitens und der universitären Lehrtätigkeit, fördern in diesem Kontext aber auch zugleich selbstausbeuterische Arbeitsverhältnisse, um im Wettbewerb um die wenigen Dauerstellen im Mittelbau und die Professuren bestmöglich aufgestellt zu sein, sofern ein Verbleib im Wissenschaftsbetrieb angestrebt wird. Gegenüber dem Berichtszeitjahr 2014 setzt sich der Trend in Richtung Teilzeitbeschäftigungsverhältnisse nicht fort und liegt nun wieder beim Ausgangsniveau von 2010 mit 58 \% (gegenüber 61 \% im Jahr 2014). Der große Anteil von Teilzeitbeschäftigungsverhältnissen spricht einerseits für eine hohe Flexibilisierung der Arbeitsverhältnisse und impliziert damit auch positive Auswirkungen auf die Work-Life-Balance und die Vereinbarkeit von Beruf und Familie. Andererseits sind damit aber auch Gehaltseinbußen verbunden. Darüber hinaus indiziert der hohe Anteil von befristeten teilzeitbeschäftigten wissenschaftlichen Mitarbeitern auch eine Zunahme der Qualifizierungsangebote für den wissenschaftlichen Nachwuchs und damit auch bessere 
Aussichten für die Nachwuchsrekrutierung. Zu hinterfragen ist, ob die Teilzeitbeschäftigung auf Wunsch der Beschäftigten erfolgt, oder darin begründet ist, dass Qualifikationsstellen oftmals nur $50 \%$ oder $65 \%$ Stellenumfang beinhalten, was aber mit den vorliegenden Daten nicht weiter untersucht werden kann. In diesem Zusammenhang spielt auch der Workload der Qualifikanten eine Rolle, der in diesem engen zeitlichen Rahmen besonders in Verbindung mit anfallenden Arbeiten im Kontext von Forschungsprojekten nicht selten nur schwerlich zu bewältigen ist, was die Zeit der Promotionsphase unverschuldet in die Länge ziehen kann. Zudem stellt sich die Frage, wo der fertig ausgebildete wissenschaftliche Nachwuchs im wissenschaftlichen System nach der Promotionsphase Fuß fassen kann, wenn nicht der selektionsreiche Weg zur Professur angestrebt wird. Hier versprechen Stellen im Bereich der Lehrtätigkeit langfristigere Perspektiven (31 \% der Stellen auf Dauer) als in der Forschung (12\% der Stellen auf Dauer). Darüber hinaus gewinnen Stellen außerhalb des Wissenschaftsbetriebs an Attraktivität für die Phase nach der Promotion.

Tabelle 4.4: Entwicklung des nebenberuflichen wissenschaftlichen Personals in der Erziehungswissenschaft (einschließlich Sonder- und Sozialpädagogik) 2006, 2010, 2014 und 2018 an wissenschaftlichen Hochschulen

\begin{tabular}{|c|c|c|c|c|c|c|c|c|c|}
\hline & \multicolumn{2}{|c|}{2006} & \multicolumn{2}{|c|}{2010} & \multicolumn{2}{|c|}{2014} & \multicolumn{2}{|c|}{2018} & \multirow{2}{*}{$\begin{array}{c}\text { Veränderung } \\
\text { 2014-2018 } \\
\text { abs. }\end{array}$} \\
\hline & abs. & $\%$ & abs. & $\%$ & abs. & $\%$ & abs. & $\%$ & \\
\hline \multicolumn{10}{|c|}{ Erziehungswissenschaft } \\
\hline Emeriti & 36 & 1,6 & 37 & 1,5 & 29 & 1,0 & 24 & 0,9 & -5 \\
\hline Lehrbeauftragte & 1.803 & 79,1 & 1.745 & 70,0 & 1.870 & 64,0 & 1.743 & 62,0 & -127 \\
\hline wiss. Hilfskräfte & 440 & 19,3 & 710 & 28,5 & 1.023 & 35,0 & 1.046 & 37,2 & +23 \\
\hline insgesamt & 2.279 & & 2.492 & & 2.922 & & 2.813 & & \\
\hline \multicolumn{10}{|c|}{ Sonderpädagogik } \\
\hline Emeriti & 2 & 0,4 & 1 & 0,2 & 1 & 0,2 & 2 & 0,3 & +1 \\
\hline Lehrbeauftrage & 428 & 87,9 & 377 & 75,7 & 460 & 82,6 & 424 & 70,7 & -36 \\
\hline wiss. Hilfskräfte & 57 & 11,7 & 120 & 24,1 & 96 & 17,2 & 174 & 29,0 & +78 \\
\hline insgesamt & 487 & & 498 & & 557 & & 600 & & \\
\hline \multicolumn{10}{|c|}{ Sozialpädagogik } \\
\hline Emeriti & 4 & 1,4 & 3 & 0,8 & 5 & 1,4 & 6 & 1,9 & +1 \\
\hline Lehrbeauftrage & 251 & 86,0 & 300 & 84,5 & 267 & 76,3 & 245 & 75,9 & -22 \\
\hline wiss. Hilfskräfte & 37 & 12,7 & 52 & 14,6 & 78 & 22,3 & 72 & 22,2 & -6 \\
\hline insgesamt & 292 & & 355 & & 350 & & 323 & & \\
\hline
\end{tabular}

Quelle: Statistisches Bundesamt, Sonderauswertungen der Hochschulpersonalstatistik, eigene Berechnungen

Nach der Expansion des nebenberuflichen Personals in den vergangenen Berichtszeiträumen ist erstmals ein Rückgang zu konstatieren (vgl. Tabelle 4.4). 
Entscheidenden Anteil daran hat insbesondere der Rückgang der Anzahl von Lehrbeauftragten in den Erziehungswissenschaften, der sich aber auch in der Sonder- und Sozialpädagogik - wenn auch auf vergleichbar niedrigem Niveau - zeigt. Dieser Trend ist erfreulich, da inadäquate Beschäftigungsverhältnisse abnehmen und zu Teilen sicher auch in hauptberuflichen Stellen als Lehrkräfte für besondere Aufgaben aufgehen, auch wenn der Aufwuchs der hauptberuflichen Stellen - wie oben gezeigt - bestenfalls als moderat beschrieben werden kann.

$\mathrm{Ob}$ mit dieser Verschiebung eine nachhaltige Absicherung der Lehre gewährleistet ist, insbesondere wenn man diesen Trend zusammen mit dem geringen Ausbau der Stellen von Lehrkräften für besondere Aufgaben sowie der Professuren und Juniorprofessuren betrachtet, bleibt abzuwarten. Die Zahlen deuten vielmehr darauf hin, dass die Kapazitäten für die Lehre stagnieren oder gar zurückgehen. Schaut man auf die steigenden Studierendenzahlen und den steigendem Bedarf an pädagogischem Personal und Lehrkräften in der Bildungspraxis, spricht dieser jüngste Trend eher gegen einen angemessenen und vorausschauenden Ausbau hinsichtlich der zukünftig erforderlichen erziehungswissenschaftlichen Lehrkapazitäten. Weitgehende Konstanz herrscht bei der Zahl der Emeriti und den wissenschaftlichen Hilfskräften.

Bei den wissenschaftlichen Hilfskräften ${ }^{5}$ gibt es einen marginalen Rückgang in der Erziehungswissenschaft und einen leichten Zuwachs in der Sozialpädagogik. Auffällig ist der Zuwachs in der Sonderpädagogik, was für einen erhöhten Forschungsbedarf oder einen angespannten Stellenmarkt spricht. Jedenfalls scheint der im vergangenen Datenreport aufgezeigte Trend zu mehr wissenschaftlichen Hilfskräften, mit Ausnahme der Sozialpädagogik, erst einmal gebremst.

Den Abschluss dieses Abschnitts bildet wie in den vergangenen Berichtszeiträumen die Entwicklung des Verhältnisses von haupt- und nebenberuflichen Stellen. Dabei erlaubt Tabelle 4.5 auch Einblicke in die Entwicklung der Subdisziplinen Sonder- und Sozialpädagogik. Generell zeigt sich eine Entwicklung hin zu weniger nebenberuflichen Beschäftigungen insbesondere für das in der Lehre tätige Personal über alle Subdisziplinen hinweg, beim in der Forschung tätigen Personal ist die Lage dagegen nicht so eindeutig. Einer Abnahme in der Erziehungswissenschaft (26,7 \% in $2014 \mathrm{zu} 23,7 \%$ in 2018) steht eine Zunahme in der Sonderpädagogik und einem gleichbleibenden Verhältnis zwischen haupt- und nebenberuflichen Personal in der Sozialpädagogik gegenüber. Der Anstieg in der Sonderpädagogik beruht auf einem Anstieg sowohl der wissenschaftlichen Hilfskräfte (+78) als auch der wissenschaftlichen Mitarbeiter (+205). In der Sozialpädagogik nahm die Anzahl der wissenschaftlichen Mitarbeiterstellen leicht zu (+19 Stellen), ebenso wie die Anzahl der wissenschaftlichen Hilfskräfte (absolut +6 Stellen). Über alle Teildisziplinen hinweg zeigt sich weiterhin ein kontinuierlicher Trend zur Abnahme des nebenberuf-

5 Nicht erfasst sind dabei studentische Hilfskräfte, die von wissenschaftlichen Hilfskräften mit Examen (mit Bachelorabschluss) vertraglich zu unterscheiden sind. 
lichen Personals im Verhältnis zum hauptberuflichen Personal, so sank dieses Verhältnis in den vergangen zwölf Jahren um zehn Prozentpunkte. In den Subdisziplinen zeigt sich für die Erziehungswissenschaft nahezu das gleiche Bild, in der Sonderpädagogik stabilisiert sich das Verhältnis bei $43 \%$ und in der Sozialpädagogik steigt das Verhältnis zugunsten den nebenberuflichen Personals um sechs Prozentpunkte an.

Tabelle 4.5: Entwicklung des hauptberuflichen und nebenberuflichen wissenschaftlichen Personals im Mittelbau der Erziehungswissenschaft 2006, 2010, 2014 und 2018

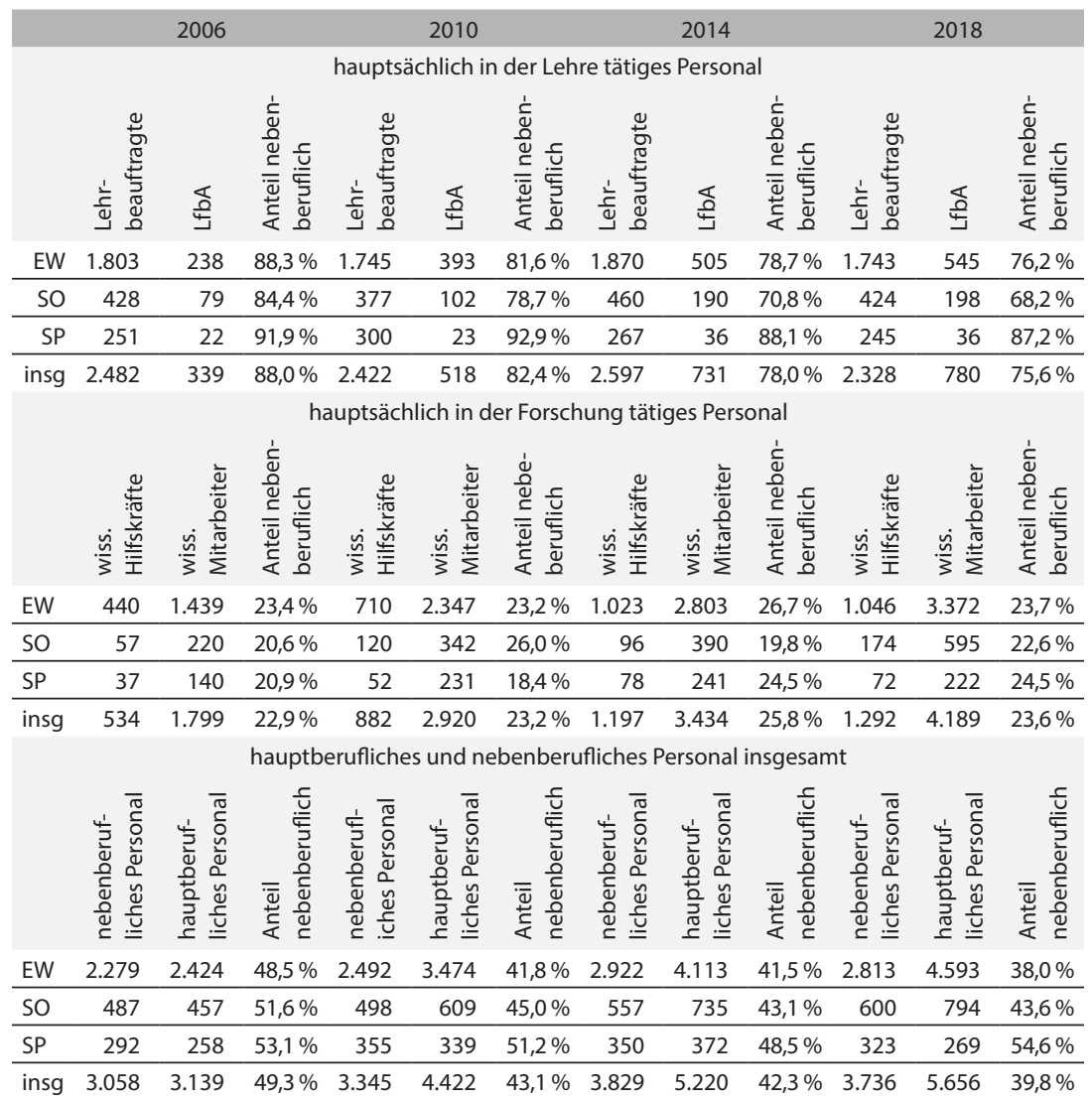

EW = Erziehungswissenschaft, $\mathrm{SO}=$ Sonderpädagogik, $\mathrm{SP}=$ Sozialpädagogik

Quelle: Statistisches Bundesamt, Sonderauswertungen der Hochschulpersonalstatistik, eigene Berechnungen 
Zusammenfassend können die referierten Zahlen als eine Entkopplung von Lehre und Forschung gedeutet werden. Die LfbA-Stellen übernehmen fast ausschließlich Lehrtätigkeiten, wobei die Lehrverpflichtungen kaum Zeit für eigenständige Forschungsaktivitäten lassen. Durch die Einführung dieser Stellen ist möglicherweise auch der Bedarf nach Lehrbeauftragten rückläufig. So besteht an den Hochschulen mittlerweile eine Personengruppe, die kaum Forschung betreibt, aber auch kaum aktuelle Erfahrungen aus der Praxis in die Lehre einfließen lassen kann und somit ausschließlich zur Erfüllung des Lehrbedarfs angestellt ist.

\subsubsection{Personalsituation im Fächervergleich}

Der Vergleich der Erziehungswissenschaften mit benachbarten Fächern erlaubt eine bessere Einschätzung der Entwicklungen hinsichtlich des Personalbestandes und der Personalstruktur. Wie in den vergangenen Berichtszeiträumen werden dazu die Wirtschaftswissenschaften sowie die Psychologie, die Politikwissenschaften und die Sozialwissenschaften herangezogen.

Die Professuren und Mitarbeiterstellen expandieren über alle Disziplinen hinweg (vgl. Tabelle 4.6). Während die Expansion der Professuren in der Psychologie, den Politikwissenschaften und in den Wirtschaftswissenschaften als echter Zuwachs zum Ausgangsniveau von 1995 konstatiert werden kann, haben die Erziehungswissenschaften das Ausgangsniveau noch nicht wieder erreicht, sind aber auf einem guten Weg. Die Sozialwissenschaften bewegen sich nun wieder leicht über dem Ausgangsniveau von 1995, die Wirtschaftswissenschaften konnten die Anzahl der Professuren seitdem um zwei Drittel steigern und liegen damit unangefochten an der Spitze des professoralen Zuwachses. Eine deutliche Stärkung hat der Mittelbau in allen Disziplinen erfahren, die Zahlen haben sich gegenüber dem Ausgangsniveau von 1995 nahezu verdoppelt, außer bei den Wirtschaftswissenschaften, deren Mitarbeiterzahl erstmals leicht um zwei Prozentpunkte zurückgeht. Rein personell in absoluten Zahlen sind die Wirtschaftswissenschaften weiterhin am stärksten aufgestellt und beschäftigen mehr Personal als die Psychologie, die Politikwissenschaften und die Sozialwissenschaften zusammen. Der Ausbau der Wirtschaftswissenschaften läuft seit 1995 relativ symmetrisch ab, was der moderate Anstieg des Verhältnisses von Mitarbeitern je Professur (von 3,3 im Jahr 1995 auf 3,49 im Jahr 2018) dokumentiert. Die anderen Disziplinen konnten dieses Verhältnis deutlich steigern, was jedoch hauptsächlich auf den starken Zuwachs im Mittelbau zurückzuführen ist und weniger auf die gestiegene Anzahl von Professuren. 
Tabelle 4.6: Entwicklung des hauptberuflichen wissenschaftlichen Personals an wissenschaftlichen Hochschulen im Fächervergleich 1995, 2000, 2005, 2010 und 2014-2018

\begin{tabular}{|c|c|c|c|c|c|c|c|c|c|c|}
\hline \multirow[t]{2}{*}{ Jahr } & \multicolumn{2}{|c|}{$\begin{array}{l}\text { Erziehungs- } \\
\text { wissenschaften }{ }^{1}\end{array}$} & \multicolumn{2}{|c|}{ Psychologie } & \multicolumn{2}{|c|}{$\begin{array}{l}\text { Politik- } \\
\text { wissenschaften }\end{array}$} & \multicolumn{2}{|c|}{$\begin{array}{l}\text { Sozial- } \\
\text { wissenschaften }\end{array}$} & \multicolumn{2}{|c|}{$\begin{array}{l}\text { Wirtschafts- } \\
\text { wissenschaften }\end{array}$} \\
\hline & $\begin{array}{l}\text { Profes- } \\
\text { soren }\end{array}$ & $\begin{array}{l}\text { Mitar- } \\
\text { beiter }^{2}\end{array}$ & $\begin{array}{l}\text { Profes- } \\
\text { soren }\end{array}$ & $\begin{array}{l}\text { Mitar- } \\
\text { beiter }^{2}\end{array}$ & $\begin{array}{l}\text { Profes- } \\
\text { soren }\end{array}$ & $\begin{array}{l}\text { Mitar- } \\
\text { beiter }^{2}\end{array}$ & $\begin{array}{l}\text { Profes- } \\
\text { soren }\end{array}$ & $\begin{array}{l}\text { Mitar- } \\
\text { beiter }\end{array}$ & $\begin{array}{l}\text { Profes- } \\
\text { soren }\end{array}$ & $\begin{array}{l}\text { Mitar- } \\
\text { beiter }^{2}\end{array}$ \\
\hline 1995 & 1.091 & 2.180 & 541 & 1.581 & 284 & 632 & 507 & 1.070 & 1.316 & 4.356 \\
\hline 2000 & 979 & 2.207 & 524 & 1.794 & 271 & 641 & 475 & 1.170 & 1.356 & 4.552 \\
\hline 2005 & 861 & 2.301 & 579 & 2.060 & 310 & 727 & 436 & 1.127 & 1.401 & 5.120 \\
\hline 2010 & 934 & 3.488 & 577 & 2.796 & 317 & 915 & 460 & 1.649 & 1.706 & 6.794 \\
\hline 2014 & 1.011 & 4.209 & 648 & 3.267 & 353 & 1.083 & 489 & 1.892 & 2.039 & 7.452 \\
\hline 2015 & 1.021 & 4.356 & 662 & 3.437 & 354 & 1.149 & 511 & 1.992 & 2.096 & 7.381 \\
\hline 2016 & 1.038 & 4.409 & 695 & 3.590 & 365 & 1.138 & 507 & 1.977 & 2.102 & 7.502 \\
\hline 2017 & 1.043 & 4.714 & 701 & 3.668 & 367 & 1.250 & 515 & 2.091 & 2.181 & 7.850 \\
\hline \multirow[t]{3}{*}{2018} & 1.045 & 5.020 & 714 & 3.875 & 370 & 1.275 & 522 & 2.188 & 2.196 & 7.666 \\
\hline & $\begin{array}{l}\text { Profes- } \\
\text { soren }\end{array}$ & $\begin{array}{l}\text { Mitar- } \\
\text { beiter }\end{array}$ & $\begin{array}{l}\text { Profes- } \\
\text { soren }\end{array}$ & $\begin{array}{l}\text { Mitar- } \\
\text { beiter }\end{array}$ & $\begin{array}{l}\text { Profes- } \\
\text { soren }\end{array}$ & $\begin{array}{l}\text { Mitar- } \\
\text { beiter }\end{array}$ & $\begin{array}{l}\text { Profes- } \\
\text { soren }\end{array}$ & $\begin{array}{l}\text { Mitar- } \\
\text { beiter }\end{array}$ & $\begin{array}{l}\text { Profes- } \\
\text { soren }\end{array}$ & $\begin{array}{l}\text { Mitar- } \\
\text { beiter }\end{array}$ \\
\hline & $\begin{array}{l}1995= \\
100\end{array}$ & je Prof. & $\begin{array}{l}1995= \\
100\end{array}$ & je Prof. & $\begin{array}{l}1995= \\
100\end{array}$ & je Prof. & $\begin{array}{l}1995= \\
100\end{array}$ & je Prof. & $\begin{array}{l}1995= \\
100\end{array}$ & je Prof. \\
\hline 1995 & 1 & 2,00 & 1 & 2,92 & 1 & 2,23 & 1 & 2,11 & 1 & 3,31 \\
\hline 2000 & 0,90 & 2,25 & 0,97 & 3,42 & 0,95 & 2,37 & 0,94 & 2,46 & 1,03 & 3,36 \\
\hline 2005 & 0,79 & 2,67 & 1,07 & 3,56 & 1,09 & 2,35 & 0,86 & 2,58 & 1,06 & 3,65 \\
\hline 2010 & 0,86 & 3,73 & 1,07 & 4,85 & 1,12 & 2,89 & 0,91 & 3,58 & 1,3 & 3,98 \\
\hline 2014 & 0,93 & 4,16 & 1,20 & 5,04 & 1,24 & 3,07 & 0,96 & 3,87 & 1,55 & 3,65 \\
\hline 2015 & 0,94 & 4,27 & 1,22 & 5,19 & 1,25 & 3,25 & 1,01 & 3,90 & 1,59 & 3,52 \\
\hline 2016 & 0,95 & 4,25 & 1,28 & 5,17 & 1,29 & 3,12 & 1 & 3,90 & 1,60 & 3,57 \\
\hline 2017 & 0,96 & 4,52 & 1,30 & 5,23 & 1,29 & 3,41 & 1,02 & 4,06 & 1,66 & 3,60 \\
\hline 2018 & 0,96 & 4,80 & 1,32 & 5,43 & 1,30 & 3,45 & 1,03 & 4,19 & 1,67 & 3,49 \\
\hline
\end{tabular}

1 einschließlich Sonder- und Sozialpädagogik

2 Als Mitarbeiter werden Assistenten und Dozenten, wissenschaftliche Mitarbeiter und Lehrkräfte für besondere Aufgaben zusammen bezeichnet.

Quelle: Statistisches Bundesamt, Hochschulstatistik, eigene Berechnungen

Weiterhin gering bleibt der Anteil an Juniorprofessuren (vgl. Tabelle 4.7) gemessen an den Gesamtprofessuren, die Werte schwanken zwischen dem niedrigsten Wert in der Politikwissenschaft (7\%) und dem höchsten Wert in den Wirtschaftswissenschaften (12,5\%). Obwohl es 2018 absolut mehr Juniorprofessuren in den Wirtschaftswissenschaften gab (287 vs. 268), sank das Verhältnis marginal aufgrund der weiter gestiegenen Zahl an Gesamtprofessuren von $13,7 \%$ auf $12,5 \%$. In der Politikwissenschaft und der Sozialwissenschaft ist die Zahl der Juniorprofessuren gar leicht rückläufig, in der Psychologie bleibt sie nahezu konstant. In den Erziehungswissenschaften ist ein Plus von 20 Junior- 
professuren zu verzeichnen, was einer Steigerung des Anteils gegenüber den Gesamtprofessuren um 1,7 \% entspricht.

Tabelle 4.7: Juniorprofessuren, Zeitprofessuren und aus Drittmitteln finanzierte Professuren an wissenschaftlichen Hochschulen im Disziplinenvergleich 2018

\begin{tabular}{|c|c|c|c|c|c|}
\hline Professuren & $\begin{array}{l}\text { Erziehungswis- } \\
\text { senschaften }^{1}\end{array}$ & Psychologie & $\begin{array}{l}\text { Politik- } \\
\text { wissenschaften }\end{array}$ & $\begin{array}{l}\text { Sozial- } \\
\text { wissenschaften }\end{array}$ & $\begin{array}{l}\text { Wirtschafts- } \\
\text { wissenschaften }\end{array}$ \\
\hline insgesamt & 1.045 & 714 & 370 & 522 & 2.196 \\
\hline \multicolumn{6}{|c|}{ Juniorprofessuren } \\
\hline absolut & 93 & 48 & 26 & 40 & 274 \\
\hline in v. $\mathrm{H}$. & 8,9 & 6,7 & 7,0 & 7,7 & 12,5 \\
\hline \multicolumn{6}{|c|}{ Professuren auf Zeitstellen ${ }^{2}$} \\
\hline absolut & 221 & 102 & 81 & 112 & 446 \\
\hline in v. H. & 21,1 & 14,3 & 21,9 & 21,4 & 20,3 \\
\hline \multicolumn{6}{|c|}{ aus Drittmitteln finanzierte Professuren } \\
\hline absolut 2006 & 8 & 4 & 1 & 6 & 34 \\
\hline 2010 & 25 & 24 & 22 & 23 & 137 \\
\hline 2014 & 51 & 40 & 20 & 23 & 146 \\
\hline 2018 & 43 & 47 & 23 & 30 & 131 \\
\hline in v. H. 2018 & 4,1 & 6,6 & 6,2 & 5,7 & 6,0 \\
\hline
\end{tabular}

1 einschließlich Sonder- und Sozialpädagogik

2 nicht alle Daten verfügbar, z.T. geheim

Quelle: Statistisches Bundesamt, Hochschulstatistik, eigene Berechnungen

Gegenüber dem vergangenen Berichtszeitraum sind die zeitlich befristeten Professuren über alle Disziplinen hinweg rückläufig, was eine sicherlich erfreuliche Entwicklung darstellt, die sich auch im Verhältnis zu den Gesamtprofessuren nachvollziehen lässt. Auch die Entwicklung von aus Drittmitteln finanzierten Professuren ist verglichen mit dem letzten Berichtszeitraum relativ konstant geblieben und in den Erziehungswissenschaften leicht rückläufig (51 Stellen im Jahr 2014 zu 43 Stellen im Jahr 2018) und liegt nun bei $4 \%$. Zuwächse gab es hier insbesondere in der Psychologie $(+18 \%$, absolut 7 Stellen) sowie bei den Sozialwissenschaften (30\%, absolut 7 Stellen) und geringe Zuwächse bei den Politikwissenschaften $(+15 \%$, absolut 3 Stellen).

Die Analyse des Mittelbaus über die Fächer hinweg (siehe Tabelle 4.8) zeigt, dass sich der Trend zu mehr wissenschaftlichem Personal auch in den anderen sozialwissenschaftlichen Disziplinen widerspiegelt, auch wenn sich die Zuwächse auf dem Ein-Prozent-Niveau gemessen am gesamten Personal des Mittelbaus im jeweiligen Fach bewegen. Nach wie vor sichtbar ist der immanente Ausbildungsauftrag für Lehrkräfte in den Erziehungs- 
wissenschaften, der sich am hohen Anteil der Lehrkräfte für besondere Aufgaben (15,5 \%) ablesen lässt und beinahe zehn Prozentpunkte über den zweitplatzierten Sozialwissenschaften liegt. Jedoch ist durch den weiter oben beschriebenen Ausbau des wissenschaftlichen Personals der Anteil an allen Stellen im Mittelbau auch in den Erziehungswissenschaften um zwei Prozentpunkte gesunken. Der Ausbau des wissenschaftlichen Personals im Mittelbau in den Erziehungswissenschaften geschieht im Rahmen befristeter Arbeitsverhältnisse, während der Anteil der Dauerstellen relativ konstant bleibt, bei einem leichten Rückgang der Dauerstellen auf Vollzeit (2014: 8,3 \%; 2018: 7,9 \%). Der größte Zuwachs findet sich bei den befristeten Vollzeitstellen (Anteil steigt um 3,4 \%) und den befristeten Teilzeitstellen (Anteil steigt um 1,4 \%). Problematisch erscheint der weiterhin hohe Anteil befristeter Arbeitsverhältnisse über alle Fächer hinweg, die zudem meist auf Teilzeitbasis laufen.

Tabelle 4.8: Struktur des Mittelbaus in der Erziehungswissenschaft und in ausgewählten Disziplinen an wissenschaftlichen Hochschulen 2018

\begin{tabular}{|c|c|c|c|c|c|}
\hline & $\begin{array}{l}\text { Erziehungs- } \\
\text { wissenschaften }\end{array}$ & Psychologie & $\begin{array}{l}\text { Politikwissen- } \\
\text { schaften }^{1}\end{array}$ & $\begin{array}{l}\text { Sozialwissen- } \\
\text { schaften }^{1}\end{array}$ & $\begin{array}{l}\text { Wirtschafts- } \\
\text { wissenschaften }\end{array}$ \\
\hline Dienstbezeichnung & $\%$ & $\%$ & $\%$ & $\%$ & $\%$ \\
\hline $\begin{array}{l}\text { Dozenten und Assis- } \\
\text { tenten insgesamt }\end{array}$ & 1 & 2,1 & 2,3 & 1,5 & 3,1 \\
\hline $\begin{array}{l}\text { wiss. Mitarbeiter } \\
\text { insgesamt }\end{array}$ & 83,4 & 95,1 & 93,2 & 93,4 & 95,3 \\
\hline Vollzeit auf Dauer & 7,9 & 6,5 & 5,5 & 6,9 & 3,4 \\
\hline Teilzeit auf Dauer & 2,2 & 3,3 & 1,4 & 2 & 0,8 \\
\hline Vollzeit auf Zeit & 25,5 & 22,3 & 27,8 & 30,4 & 39,7 \\
\hline Teilzeit auf Zeit & 47,8 & 63,2 & 58,4 & 54,1 & 51,4 \\
\hline LfbA insgesamt & 15,5 & 2,7 & 4,5 & 6,1 & 1,5 \\
\hline Vollzeit auf Dauer & 3,3 & 0,6 & k.A. & k.A. & 0,7 \\
\hline Teilzeit auf Dauer & 1,6 & 0,5 & k.A. & k.A. & 0,2 \\
\hline Vollzeit auf Zeit & 4,1 & 0,6 & k.A. & k.A. & 0,1 \\
\hline Teilzeit auf Zeit & 6,5 & 1 & k.A. & k.A. & 0,5 \\
\hline insgesamt absolut & 5.020 & 3.875 & 1.275 & 2.188 & 7.666 \\
\hline
\end{tabular}

1 Leider wurde für dieses Fach für den Zeitraum 2018 keine weitere Differenzierung der Beschäftigungsverhältnisse für die LfbA in der Hochschulstatistik ausgewiesen.

Quelle: Statistisches Bundesamt, Hochschulstatistik, eigene Berechnungen

Die im vergangenen Beobachtungszeitraum konstatierte Eigenentwicklung der Erziehungswissenschaften durch die massive Zunahme von Lehrkräften mit besonderen Aufgaben setzt sich nicht fort. Wie in den Abschnitten 4.1.1 und 4.1.2 gezeigt, begrenzt sich der weitere Ausbau in diesem Berichtszeitraum vornehmlich auf das wissenschaftlich tätige Personal und die Professuren. 
Tabelle 4.9: Entwicklung der Zahl der wissenschaftlichen Hilfskräfte im Disziplinenvergleich an wissenschaftlichen Hochschulen 2006, 2010, 2014 und 2018

\begin{tabular}{|c|c|c|c|c|c|c|c|c|c|}
\hline Disziplin & $\begin{array}{l}\text { wiss. } \\
\text { Hilfs- } \\
\text { kräfte }\end{array}$ & $\begin{array}{l}06 \\
\text { Relation } \\
\text { zu wiss. } \\
\text { Mitar- } \\
\text { beitern }\end{array}$ & $\begin{array}{l}\text { wiss. } \\
\text { Hilfs- } \\
\text { kräfte }\end{array}$ & $\begin{array}{l}10 \\
\text { Relation } \\
\text { zu wiss. } \\
\text { Mitar- } \\
\text { beitern }\end{array}$ & $\begin{array}{l}\text { wiss. } \\
\text { Hilfs- } \\
\text { kräfte }\end{array}$ & $\begin{array}{l}14 \\
\text { Relation } \\
\text { zu wiss. } \\
\text { Mitar- } \\
\text { beitern }\end{array}$ & $\begin{array}{l}\text { wiss. } \\
\text { Hilfs- } \\
\text { kräfte }\end{array}$ & $\begin{array}{l}18 \\
\text { Relation } \\
\text { zu wiss. } \\
\text { Mitar- } \\
\text { beitern }\end{array}$ & $\begin{array}{l}\text { Verände- } \\
\text { rung } \\
\text { 2014-2018 } \\
\text { abs. }\end{array}$ \\
\hline $\begin{array}{l}\text { Erziehungs- } \\
\text { wissenschaft }\end{array}$ & 537 & 0,30 & 882 & 0,30 & 1.197 & 0,35 & 1.292 & 0,31 & +95 \\
\hline Psychologie & 363 & 0,19 & 547 & 0,21 & 1.299 & 0,42 & 1.251 & 0,34 & -48 \\
\hline $\begin{array}{l}\text { Politik- } \\
\text { wissenschaft }\end{array}$ & 201 & 0,37 & 324 & 0,39 & 466 & 0,47 & 446 & 0,38 & -20 \\
\hline $\begin{array}{l}\text { Sozial- } \\
\text { wissenschaft }\end{array}$ & 329 & 0,33 & 460 & 0,31 & 688 & 0,40 & 625 & 0,31 & -63 \\
\hline $\begin{array}{l}\text { Wirtschafts- } \\
\text { wissenschaft }\end{array}$ & 685 & 0,14 & 1365 & 0,21 & 2.755 & 0,39 & 2.908 & 0,40 & +153 \\
\hline
\end{tabular}

Quelle: Statistisches Bundesamt, Sonderauswertungen der Hochschulpersonalstatistik, eigene Berechnungen

Der im letzten Berichtszeitraum konstatierte Zuwachs an wissenschaftlichen Hilfskräften ist über die Fächergruppen hinweg gebremst. In der Psychologie, den Politikwissenschaften und den Sozialwissenschaften ist der Trend sogar eher rückläufig. Moderate Zuwächse verzeichnen die Erziehungswissenschaften und die Wirtschaftswissenschaften, die weiterhin expandieren. In den letzten elf Jahren hat sich die Zahl der wissenschaftlichen Hilfskräfte in den Erziehungswissenschaften, den Politikwissenschaften und den Sozialwissenschaften nahezu verdoppelt, in der Psychologie verdreifacht und in den Wirtschaftswissenschaften sogar mehr als vervierfacht. Diese Entwicklung lässt auf gestiegene Forschungsanstrengungen in den Disziplinen schließen, was einerseits zu begrüßen ist, andererseits aber weiter auf eine hohe Zahl inadäquater Beschäftigungsverhältnisse im Wissenschaftsbetrieb nach dem Abschluss des Studiums schließen lässt. Immanent ist diesen Zahlen wohl der vermehrte Wettbewerb um forschungsgebundene Drittmittel (siehe Kapitel 5.1), die sowohl die Erziehungswissenschaften selbst als auch die Vergleichsdisziplinen betreffen. 


\subsection{Personalentwicklung im Fach Erziehungswissenschaft aus der Perspektive von Stellenausschreibungen}

Die Personalentwicklung im Fach Erziehungswissenschaft im Zeitraum 2015 bis 2018 soll im folgenden Abschnitt über eine Analyse der Stellenausschreibungen (ohne Fachdidaktik) für Professuren im Bereich Erziehungswissenschaft und Sozialpädagogik an wissenschaftlichen Hochschulen sowie an Fachhochschulen dargestellt werden. Dabei stützen wir uns auf eine vollständige Auswertung aller Ausschreibungen von erziehungswissenschaftlichen Professuren an wissenschaftlichen Hochschulen in diesem Zeitraum. Zudem wurden die im gleichen Zeitraum an staatlichen und privaten Fachhochschulen ausgeschriebenen Professuren für Sozialpädagogik/Soziale Arbeit mit berücksichtigt, da Stellen in der Hochschullehre an Fachhochschulen eine zusätzliche berufliche Möglichkeit für den promovierten erziehungswissenschaftlichen Nachwuchs mit einer mindestens dreijährigen beruflichen Praxis außerhalb der Hochschule bieten. Durchgesehen und analysiert wurden die Stellenausschreibungen jeder Ausgabe der Zeit und der Deutschen Universitätszeitung (DUZ) im Zeitraum von Januar 2015 bis Dezember 2018. Dabei wurden Professuren, die erkennbar mehrfach ausgeschrieben wurden, nur einmal gezählt.

Aufgrund der Anknüpfung an die Berechnungen von Rauschenbach/Christ 1994; Krüger/Weishaupt 2000; Krüger/Schmidt/Siebholz/Weishaupt 2004; Krüger/Schnoor/Weishaupt 2008; Krüger/Kücker/Weishaupt 2012 und Gerecht/ Krüger/Post/Weishaupt 2016, die bereits Stellenausschreibungen für die Zeiträume von Oktober 1990 bis September 1993, von Oktober 1993 bis Dezember 1998, von Anfang 1999 bis Ende 2002, von Beginn 2003 bis Ende 2006, von Anfang 2007 bis Ende 2010 und von Januar 2011 bis Dezember 2014 untersucht haben, ist es zudem möglich, die personelle sowie disziplinäre Entwicklung der Erziehungswissenschaft - soweit sie sich an Stellenausschreibungen festmachen lässt - über einen Zeitraum von fast 28 Jahren, von Oktober 1990 bis Dezember 2018, auch unter Berücksichtigung des personellen Neuaufbaus und der aktuellen Veränderungen in den neuen Bundesländern zu untersuchen.

\subsubsection{Gesamtüberblick über die ausgeschriebenen erziehungswissenschaftlichen Professuren}

Wie sehen nun die aktuellen Entwicklungen aus? Insgesamt wurden im Untersuchungszeitraum von Januar 2015 bis Dezember 2018576 Professuren (ohne Fachdidaktik und Juniorprofessuren) im Fach Erziehungswissenschaft ausgeschrieben (vgl. Tabelle 4.10). Darunter sind 500 Professuren in den alten Bundesländern, davon 187 Stellen an wissenschaftlichen Hochschulen und 313 an Fachhochschulen, und 76 in den neuen Bundesländern, davon 28 Stellen an wissenschaftlichen Hochschulen und 48 an Fachhochschulen, ausgeschrieben worden. 
Damit ist das Gesamtvolumen der Stellenausschreibungen im Vergleich zu dem von Gerecht, Krüger, Post und Weishaupt (2016: 150) für den vorherigen Untersuchungszeitraum von 2011-2014 herausgearbeiteten 778 Stellen deutlich zurückgegangen.

Tabelle 4.10: Ausgeschriebene Professuren in Erziehungswissenschaft (ohne Fachdidaktik und ohne Juniorprofessuren) und Sozialpädagogik nach Art der Hochschule und alten und neuen Bundesländern (2015-2018)

\begin{tabular}{lrrrrrrr} 
& \multicolumn{2}{c}{$\begin{array}{r}\text { wissenschaftliche } \\
\text { Hochschulen }\end{array}$} & \multicolumn{2}{c}{ Fachhochschulen } & \multicolumn{2}{c}{$\begin{array}{c}\text { Hochschulen } \\
\text { insgesamt }\end{array}$} \\
& abs. & $\%$ & abs. & $\%$ & abs. & $\%$ \\
\hline alte Bundesländer & 187 & 32,47 & 313 & 54,34 & 500 & 86,81 \\
\hline neue Bundesländer & 28 & 4,86 & 48 & 8,33 & 76 & 13,19 \\
insgesamt & 215 & 37,77 & 361 & 62,67 & 576 & 100,00
\end{tabular}

Quelle: Deutsche Universitätszeitung; Die Zeit; eigene Berechnungen

Fasst man die Ergebnisse unserer aktuellen Erhebung mit den in den Vorgängerstudien (Rauschenbach/Christ 1994; Krüger/Weishaupt 2000; Krüger/ Schmidt/Siebholz/Weishaupt 2004; Krüger/Schnoor/Weishaupt 2008; Krüger/ Kücker/Weishaupt 2012; Gerecht/Krüger/Post/Weishaupt 2016) herausgearbeiteten Daten zusammen, so ergibt sich ein Gesamtvolumen von 4.127 Stellenausschreibungen an Universitäten und Fachhochschulen in einem Zeitraum von fast 28 Jahren (vgl. Tabelle 4.11), was schon etwas über der maximalen Besetzungszeit einer Professur liegt und somit Neu- und Wiederbesetzungen von Stellen impliziert.

Tabelle 4.11: Ausgeschriebene Professuren in Erziehungswissenschaft (ohne Fachdidaktik und ohne Juniorprofessuren) und Sozialpädagogik nach Art der Hochschule und alten und neuen Bundesländern $(1990-2018 ; n=4.127)$

\begin{tabular}{lrrrrcr} 
& \multicolumn{2}{c}{$\begin{array}{c}\text { wissenschaftliche } \\
\text { Hochschulen }\end{array}$} & \multicolumn{2}{c}{ Fachhochschulen } & \multicolumn{2}{c}{$\begin{array}{c}\text { Hochschulen } \\
\text { insgesamt }\end{array}$} \\
& abs. & $\%$ & abs. & $\%$ & abs. & $\%$ \\
\hline alte Bundesländer & 1.777 & 43,06 & 1.503 & 36,42 & 3.280 & 79,48 \\
\hline neue Bundesländer & 406 & 9,84 & 401 & 10,69 & 847 & 20,52 \\
insgesamt & 2.183 & 52,90 & 1.944 & 47,10 & 4.127 & 100,00
\end{tabular}

Quelle: Deutsche Universitätszeitung; Die Zeit; Rauschenbach/Christ 1994; Krüger/Weishaupt 2000; Krüger/Schmidt/Siebholz/Weishaupt 2004; Krüger/Schnoor/Weishaupt 2008; Krüger/ Kücker/Weishaupt 2012; Gerecht/Krüger/Post/Weishaupt 2016, eigene Berechnungen 
Es wurden 2.183 Stellen für Professuren im Fach Erziehungswissenschaft (ohne Fachdidaktik und Juniorprofessuren) von Oktober 1990 bis Dezember 2018 an wissenschaftlichen Hochschulen, 1.777 in Westdeutschland und 406 im Osten des Landes, sowie 1.944 Stellen für erziehungswissenschaftliche Professuren an Fachhochschulen, darunter 1.503 Stellen in den alten und 441 Professuren in den neuen Bundesländern, erfasst.

Vergleicht man das Ausschreibungsvolumen nur an wissenschaftlichen Hochschulen mit den Daten der amtlichen Statistik, so ist dieses mit etwa 2.183 Stellen mehr als doppelt so hoch wie die im Jahr 2018 im Fach Erziehungswissenschaft registrierten Professorenstellen. Dieses hohe Ausschreibungsvolumen erklärt sich erstens dadurch, dass der gesamte Neuaufbau der Erziehungswissenschaft in den neuen Bundesländern in diesem Zeitraum realisiert worden ist, zweitens in den alten Bundesländern seit Mitte der 1990er Jahre und auch in den neuen Bundesländern seit Mitte der 2000er Jahre eine Pensionierungswelle eingesetzt hat, drittens gerade in den vergangenen Jahren wieder die Tendenz einer Zunahme bei den Professuren im Fach Erziehungswissenschaft festgestellt werden kann (vgl. Abschnitt 4.1.1) und viertens in dem untersuchten Zeitraum von fast 28 Jahren vermutlich eine Vielzahl von Stellenwechseln von C3- auf C4-Stellen bzw. W2- auf W3-Stellen stattgefunden haben und eine ganze Reihe von Stellen inzwischen auch bereits in der zweiten Generation neu besetzt worden sind. Da diese Gründe für diese Ausschreibungen den Ausschreibungstexten selbst nicht zu entnehmen sind, ist ein direkter Vergleich des Ausschreibungsvolumens mit den in der amtlichen Statistik sich dokumentierenden Veränderungen jedoch nur begrenzt möglich.

\subsubsection{Erziehungswissenschaftliche Professuren in verschiedenen Subdisziplinen}

Um die Entwicklungen innerhalb der Hochschulen zu untersuchen, ist es nötig, die Entwicklungen der einzelnen Subdisziplinen in der Erziehungswissenschaft genauer zu betrachten. Ein Blick auf das Innenverhältnis zwischen den Teilgebieten an allen Hochschulen der alten und neuen Bundesländer einschließlich der Fachhochschulen macht im Zeitraum zwischen 2015 und 2018 folgende Trends bei den Stellenausschreibungen deutlich:

Den größten Anteil am Gesamtvolumen nimmt, wie in vorherigen Untersuchungszeiträumen, die Sozialpädagogik/Soziale Arbeit ein, was sich durch die Einbeziehung der Fachhochschulen in die Berechnungen der Tabelle 4.12 ergibt. Im Vergleich zu dem Untersuchungszeitraum 2011 bis 2014 ist das Ausschreibungsvolumen mit 190 Professuren sogar noch mal leicht angestiegen, was möglicherweise auf eine Vielzahl von Stellenausschreibungen durch neu gegründete (internationale) privat organisierte Hochschulen, oftmals mit dualen Studiengängen, an zahlreichen Standorten zurückzufüh- 
ren ist. Rechnet man die Stellenausschreibungen für die Pädagogik der frühen Kindheit, die seit 2003 erstmals mit erfasst wurden und die in den vergangenen vier Jahren 25 Stellen umfassten, zu denen der Sozialpädagogik/ Sozialen Arbeit hinzu, so machen diese weit über 40 \% der an Hochschulen ausgeschriebenen Stellen aus.

Tabelle 4.12: Ausgeschriebene Professuren in Erziehungswissenschaft (ohne Fachdidaktik und ohne Juniorprofessuren) und Sozialpädagogik an Hochschulen (einschl. Fachhochschulen) nach Fachgebieten und alten und neuen Bundesländern (2015-2018; n=576)

\begin{tabular}{|c|c|c|c|c|c|c|}
\hline \multirow[b]{2}{*}{ Fachrichtungen/Teilgebiete } & \multicolumn{2}{|c|}{$\begin{array}{l}\text { alte } \\
\text { Bundesländer }\end{array}$} & \multicolumn{2}{|c|}{$\begin{array}{l}\text { neue } \\
\text { Bundesländer }\end{array}$} & \multicolumn{2}{|c|}{ insgesamt } \\
\hline & abs. & $\%$ & abs. & $\%$ & abs. & $\%$ \\
\hline Allgemeine Pädagogik & 42 & 8,40 & 4 & 5,26 & 46 & 7,99 \\
\hline Didaktik/Schulpädagogik & 18 & 3,60 & 2 & 2,61 & 20 & 3,47 \\
\hline $\begin{array}{l}\text { empirische Bildungsforschung/ } \\
\text { emp. Schul-/Unterrichtsforschung }\end{array}$ & 47 & 9,40 & 4 & 5,26 & 51 & 8,85 \\
\hline Sonderpädagogik & 48 & 9,60 & 11 & 14,47 & 59 & 10,24 \\
\hline Sozialpädagogik/Soziale Arbeit & 190 & 38,00 & 32 & 43,42 & 222 & 38,54 \\
\hline Pädagogik der frühen Kindheit & 25 & 5,00 & 8 & 10,53 & 33 & 5,73 \\
\hline Erwachsenenbildung & 8 & 1,60 & 2 & 2,63 & 10 & 1,74 \\
\hline Berufs- und Wirtschaftspädagogik & 22 & 4,40 & 4 & 5,26 & 26 & 4,51 \\
\hline Medienpädagogik & 7 & 0,12 & 1 & 1,32 & 8 & 1,39 \\
\hline Kulturpädagogik & 9 & 1,80 & 1 & 1,32 & 10 & 1,74 \\
\hline Pädagogik mit Soziologie/Psychologie & 32 & 6,40 & 1 & 1,32 & 33 & 5,73 \\
\hline Pflegepädagogik/Gesundheitserziehung & 16 & 3,20 & 0 & 0,00 & 16 & 2,78 \\
\hline Recht/Verwaltung in Soz. Arbeit & 36 & 7,20 & 5 & 6,58 & 41 & 7,12 \\
\hline Sonstige & 0 & 0.00 & 1 & 1,32 & 1 & 0,17 \\
\hline insgesamt & 500 & 100,0 & 76 & 100,0 & 576 & 100,0 \\
\hline
\end{tabular}

Quelle: Deutsche Universitätszeitung; Die Zeit; eigene Berechnungen

Klammert man allerdings die Stellenausschreibungen an Fachhochschulen aus den Berechnungen aus (vgl. Tabelle 4.13) und betrachtet man nur die Stellenausschreibungen an wissenschaftlichen Hochschulen, so ergibt sich ein wesentlich geringerer Anteil der Sozialpädagogik am Gesamtvolumen, der nur 6 \% ausmacht. Noch bescheidener fällt dann zudem das Ausschreibungsvolumen für Professuren im Bereich der Pädagogik der frühen Kindheit aus, das im Untersuchungszeitraum zwischen 2015 und 2018 an wissenschaftlichen Hochschulen nur 4 Stellen umfasst. 
Tabelle 4.13: Ausgeschriebene Professuren in Erziehungswissenschaft (ohne Fachdidaktik und ohne Juniorprofessuren) an wissenschaftlichen Hochschulen nach Fachgebieten und alten und neuen Bundesländern $(2015-2018 ; n=215)$

\begin{tabular}{|c|c|c|c|c|c|c|}
\hline & \multicolumn{2}{|c|}{$\begin{array}{c}\text { alte } \\
\text { Bundesländer }\end{array}$} & \multicolumn{2}{|c|}{$\begin{array}{c}\text { neue } \\
\text { Bundesländer }\end{array}$} & \multicolumn{2}{|c|}{ insgesamt } \\
\hline & abs. & $\%$ & abs. & $\%$ & abs. & $\%$ \\
\hline Allgemeine Pädagogik & 25 & 13,37 & 4 & 14,29 & 29 & 13,49 \\
\hline Didaktik/Schulpädagogik & 17 & 9,09 & 2 & 7,14 & 19 & 8,84 \\
\hline \multicolumn{7}{|l|}{ davon: } \\
\hline Allgemeine Didaktik/Schulpädagogik & 6 & - & 1 & - & 7 & - \\
\hline Primarstufe & 9 & - & 1 & - & 10 & - \\
\hline Sekundarstufe I & 0 & - & 0 & - & 0 & - \\
\hline Sekundarstufe II & 1 & - & 0 & - & 1 & - \\
\hline schulstufenübergreifend & 1 & - & 0 & - & 1 & - \\
\hline $\begin{array}{l}\text { empirische Bildungsforschung/ } \\
\text { emp. Schul-/Unterrichtsforschung }\end{array}$ & 46 & 24,40 & 3 & 10,71 & 49 & 22,79 \\
\hline Sonderpädagogik & 34 & 18,18 & 8 & 28,57 & 42 & 19,53 \\
\hline Sozialpädagogik/Soziale Arbeit & 9 & 4,81 & 4 & 14,29 & 13 & 6,05 \\
\hline Pädagogik der frühen Kindheit & 3 & 1,60 & 1 & 3,57 & 4 & 1,86 \\
\hline Erwachsenenbildung/Weiterbildung & 7 & 3,74 & 2 & 7,14 & 9 & 4,19 \\
\hline Berufs- und Wirtschaftspädagogik & 18 & 9,63 & 3 & 10,71 & 21 & 9,77 \\
\hline Medienpädagogik & 7 & 3,74 & 1 & 3,57 & 8 & 3,72 \\
\hline Kulturpädagogik & 5 & 2,67 & 0 & 0,00 & 5 & 2,33 \\
\hline Pädagogik mit Soziologie/Psychologie & 9 & 4,81 & 0 & 0,00 & 9 & 4,19 \\
\hline Pflegepädagogik/Gesundheitserziehung & 4 & 2,14 & 1 & 0,00 & 5 & 2,33 \\
\hline Recht/Verwaltung in Soz. Arbeit & 3 & 1,60 & 0 & 0,00 & 3 & 1,40 \\
\hline Sonstige & 0 & 0,00 & 0 & 0,00 & 0 & 0,00 \\
\hline insgesamt & 187 & 100 & 28 & 100 & 215 & 100 \\
\hline
\end{tabular}

Quelle: Deutsche Universitätszeitung; Die Zeit; eigene Berechnungen

Den Spitzenplatz bei den Ausschreibungen an wissenschaftlichen Hochschulen nimmt im Untersuchungszeitraum zwischen 2015 und 2018 erstmals die empirische Bildungsforschung/empirische Schul-/Unterrichtsforschung mit gut $22 \%$ am Gesamtvolumen aller Stellen ein, während die Allgemeine Didaktik/Schulpädagogik im Vergleich zu den vorherigen Untersuchungszeiträumen deutlich an Gewicht verloren hat. Dies erklärt sich auch dadurch, dass bei den Ausschreibungen für Schulpädagogikstellen vermutlich bedingt durch die Qualitätsoffensive Lehrerbildung und veränderte Voraussetzungen der Forschungsförderung der Forschungsbezug inzwischen schon in den Ausschreibungen deutlich hervorgehoben wird. Da wir die Stellen für empirische Bildungsforschung/empirische Schul- und Unterrichtsforschung seit 2003 separat erfasst haben, können wir zudem aufzeigen, dass die empirische Bildungs- 
forschung mit insgesamt 189 ausgeschriebenen Stellen im Zeitraum zwischen 2003 und 2018 nicht nur auf der Ebene der Forschungsförderung beim Bundesministerium für Bildung und Forschung und bei der DFG, sondern auch bei den ausgeschriebenen Stellen an wissenschaftlichen Hochschulen eine enorme Konjunktur erlebt hat (vgl. Krüger/Schnorr/Weishaupt 2008; Krüger/Kücker/ Weishaupt 2012; Gerecht/Krüger/Post/Weishaupt 2016).

Einen weiteren Spitzenplatz nehmen im Berichtszeitraum von 2015 bis 2018 die Stellen im Bereich der Sonderpädagogik mit einem Fünftel des gesamten Ausschreibungsvolumens ein. Die bildungspolitische Inklusionsdebatte hat sich somit auf die Stellenausschreibungen in dieser Subdisziplin positiv ausgewirkt. Darüber hinaus sind sogar einige Stellen für allgemeine Fragen der Inklusion in der Lehrerausbildung an wissenschaftlichen Hochschulen neu eingerichtet worden und in einer Reihe von Ausschreibungen wird das Stichwort der Inklusion nun mit dem jeweils spezifischen sonderpädagogischen Förderschwerpunkt verbunden.

Relativ stabil geblieben im Vergleich zum Untersuchungszeitraum von 2011 bis 2014 ist auch das Ausschreibungsvolumen an Professuren in der Allgemeinen Pädagogik, die im aktuellen Berichtszeitraum mit einem Anteil von mehr als $13 \%$ am Gesamtvolumen aller Stellen beteiligt ist. Relativierend muss man hier allerdings konstatieren, dass sich die hier erfassten 29 Stellen auf die Bereiche der Systematischen Pädagogik, der Historischen Pädagogik, der Vergleichenden Erziehungswissenschaft, der Interkulturellen Pädagogik und der Erziehungswissenschaft im Kontext von Heterogenität und Differenz verteilen. Bei der genaueren inhaltlichen Analyse dieser Stellenausschreibungen fällt zudem auf, dass bei einigen dieser Stellen - ähnlich wie übrigens in der Schulpädagogik und Schulforschung - mit neuen Leitbegriffen wie Inklusion oder Umgang mit Differenz und Heterogenität auf aktuelle gesellschafts- und bildungspolitische Entwicklungen reagiert wird. Im Gegensatz dazu scheinen sich die aktuellen gesellschaftlichen Tendenzen der Digitalisierung und Mediatisierung aller Lebensbereiche auf das Stellenprofil des Faches Erziehungswissenschaft bislang kaum ausgewirkt zu haben, da Stellenausschreibungen in der Subdisziplin der Medienpädagogik ähnlich wie auch schon in den bisherigen Berichtszeiträumen nur einen marginalen Stellenwert einnehmen.

Vergleicht man die Anzahl der an wissenschaftlichen Hochschulen im Fach Erziehungswissenschaft im Zeitraum zwischen 2015 und 2018 insgesamt ausgeschriebenen Stellen, so ist diese im Vergleich zum Untersuchungszeitraum von 2011 bis 2014 von 363 auf 187 Stellen deutlich zurückgegangen (vgl. Gerecht/Krüger/Post/Weishaupt 2016: 153). Ein Aufwuchs an Stellen ist nur in der empirischen Bildungs-/Schul- und Unterrichtsforschung zu konstatieren, während in allen anderen Subdisziplinen teilweise sogar massive Rückgänge zu verzeichnen sind. Ob es sich dabei um einen einmaligen Vorgang oder einen längerfristigen Trend handelt, wird im Weiteren diskutiert. 
Im Folgenden soll nun im Zeitvergleich die Entwicklung der Erziehungswissenschaft durch die Untersuchung von Fachgebieten in Anlehnung an die Ergebnisse der Studie von Baumert und Roeder aus dem Jahr 1987 sowie die Berechnungen von Oktober 1990 bis Dezember 2018 untersucht werden. Da von Baumert und Roeder (1994: 31) der Bestand und die Struktur des wissenschaftlichen Personals in der Erziehungswissenschaft anhand der Vorlesungsund Personalverzeichnisse erhoben wurde, lässt der Vergleich mit der sich in den Ausschreibungen dokumentierenden Struktur aufgrund der unterschiedlichen Datensorten nur einige globale Trendaussagen zu (vgl. Tabelle 4.14):

Tabelle 4.14: Ausgeschriebene Professuren in Erziehungswissenschaft (ohne Fachdidaktik und ohne Juniorprofessuren) an wissenschaftlichen Hochschulen nach Fachgebieten und alten und neuen Bundesländern (Oktober 1990 bis Ende 2018; $\mathrm{n}=1.840$ )

\begin{tabular}{|c|c|c|c|c|c|c|c|}
\hline \multirow[b]{2}{*}{ Fächergruppen } & \multirow{2}{*}{$\begin{array}{c}\text { Baumert/ } \\
\text { Roeder } \\
\%\end{array}$} & \multicolumn{2}{|c|}{$\begin{array}{c}\text { alte } \\
\text { Bundesländer }\end{array}$} & \multicolumn{2}{|c|}{$\begin{array}{c}\text { neue } \\
\text { Bundesländer }\end{array}$} & \multicolumn{2}{|c|}{ insgesamt } \\
\hline & & abs. & $\%$ & abs. & $\%$ & abs. & $\%$ \\
\hline Allgemeine Pädagogik ${ }^{1}$ & 32,3 & 284 & 18,96 & 51 & 14,91 & 335 & 18,40 \\
\hline Schulpädagogik ${ }^{2}$ & 33,8 & 545 & 36,38 & 107 & 31,29 & 652 & 35,43 \\
\hline Sonderschulpädagogik & 14,4 & 248 & 16,56 & 80 & 23,39 & 328 & 15,54 \\
\hline Außerschulische Pädagogik ${ }^{3}$ & 19,4 & 421 & 28,10 & 104 & 30,41 & 525 & 28,53 \\
\hline insgesamt $^{4}$ & 100,0 & 1.498 & 100,0 & 342 & 100,0 & 1.840 & 100,0 \\
\hline
\end{tabular}

1 Hierin enthalten sind Stellen der Allgemeinen, Historischen, Vergleichenden und Systematischen Pädagogik.

2 Hierin enthalten sind Stellen der Schulpädagogik und der Bildungsforschung.

3 Hierin enthalten sind Stellen der Sozialpädagogik (inkl. Recht/Verwaltung in der Sozialen Arbeit), der Erwachsenenbildung, der Berufspädagogik, der Pflegepädagogik sowie der Medien- und Kulturpädagogik.

4 Hierin sind Stellen für Fachdidaktik, für Erziehungssoziologie, für Pädagogische Psychologie sowie fachlich nicht eindeutig zuzuordnende Ausschreibungen nicht enthalten.

Quelle: Deutsche Universitätszeitung; Die Zeit; Baumert/Roeder 1994; Rauschenbach/Christ 1994; Krüger/Weishaupt 2001; Krüger/Schmidt/Siebholz/Weishaupt 2004; Krüger/Schnoor/ Weishaupt 2008; Krüger/Kücker/Weishaupt 2012; Gerecht/Krüger/Post/Weishaupt 2016; eigene Berechnungen

Einen besonders großen Expansionsschub hat die Außerschulische Pädagogik seit der Erhebung 1987 von Baumert und Roeder erfahren, mit dem das Fach Erziehungswissenschaft auf die Expansion der Studierendenzahlen in den erziehungswissenschaftlichen Hauptfachstudiengängen reagiert hat. In der Allgemeinen Pädagogik hingegen zeichnet sich seit 1987 ein deutlicher Abwärtstrend durch den Verlust von etwa 14 Prozentpunkten ab. Bei den beiden anderen Fachgebieten, der Sonderpädagogik und der Schulpädagogik, lassen sich weniger große Unterschiede im Vergleich zum ursprünglichen Erhebungszeitpunkt vor 32 Jahren ablesen. Berücksichtigt man jedoch, dass in die Professuren für die Schulpädagogik auch 
jene inzwischen 189 Stellen für empirische Bildungsforschung/Schul- und Unterrichtsforschung mit einbezogen worden sind, so lässt sich für die Teildisziplin der Schulpädagogik feststellen, dass sich deren quantitatives Gewicht im Spektrum der erziehungswissenschaftlichen Subdisziplinen in den vergangenen zwei Jahrzehnten nicht gravierend verändert hat. Gleichzeitig lässt sich jedoch inzwischen eine deutliche inhaltliche und methodische Profilverschiebung konstatieren, da rund ein Drittel der ausgeschriebenen Stellen in der Schulpädagogik einen Profilschwerpunkt in der empirischen Bildungsforschung bzw. der empirischen Schul- und Unterrichtsforschung haben. Überraschend ist zudem der in den vergangenen zwei Jahrzehnten relativ stabile Trend in den Stellenausschreibungen für die Außerschulische Pädagogik. Trotz eines gravierenden Anstiegs der Lehramtsstudierenden und der gestiegenen Lehrbelastungen durch die modularisierten Lehramtsstudiengänge ist der prozentuale Anteil der Stellen für die Außerschulische Pädagogik in Gestalt vor allem der Erwachsenbildung und der Sozialpädagogik am Gesamtvolumen der Stellen an wissenschaftlichen Hochschulen kaum zurückgegangen. Dies kann auch über den nach wie vor stetig wachsenden Bedarf an Stellen im Bereich der Außerschulischen Pädagogik erklärt werden - etwa im Bereich der Kinder und Jugendhilfe (s. hierzu: Arbeitsgruppe Kinder- und Jugendhilfestatistik 2019).

\subsubsection{Bilanz und Nachwuchschancen}

In diesem Abschnitt soll nun die Entwicklung des Stellenausschreibungsvolumens der Professuren im Fach Erziehungswissenschaft (ohne Fachdidaktik und Juniorprofessuren) und im Bereich Sozialpädagogik an wissenschaftlichen Hochschulen und Fachhochschulen auf einer Zeitachse von 1991 bis 2018 in vierjährigen Zeiträumen untersucht werden (vgl. Abbildung 4.1).

Aus dieser Übersicht werden drei Entwicklungstrends deutlich. Erstens zeigt sich, dass im Vergleich zum ersten Untersuchungszeitraum von 1991 bis 1994 das ausgeschriebene Stellenvolumen in den neuen Bundesländern in den folgenden Untersuchungszeiträumen deutlich rückläufig und erst im Beobachtungszeitraum von 2007 bis 2014 wieder angestiegen ist. Dies ist wenig überraschend, da der Neuaufbau der erziehungswissenschaftlichen Fachbereiche in den neuen Bundesländern in der ersten Hälfte der 1990er Jahre realisiert worden ist und erst seit Mitte der 2000er Jahre aufgrund von Pensionierungen Neuausschreibungen wieder verstärkt eingesetzt hat. Zweitens wird deutlich, dass ab dem ersten Untersuchungszeitraum von 1991 bis 1994 und in den folgenden Untersuchungszeiträumen jeweils über 300 Professuren in den alten Bundesländern ausgeschrieben worden sind und dass sich dieses Ausschreibungsvolumen im Berichtszeitraum zwischen 2007 und 2014 noch einmal verdoppelt hat. Diese Befunde sind insofern nicht überraschend als Krüger/Schnoor/Weishaupt (2008: 111) in ihrer Prognose von 2008 bereits darauf hingewiesen haben, dass von 2007 bis $201560 \%$ der Professuren in der Erziehungswissenschaft an wissenschaftlichen Hochschulen 
frei werden. Drittens zeigt sich im aktuellen Berichtszeitraum, dass das Volumen der Stellenausschreibungen in der Zeit zwischen 2015 und 2018 zwar zurückgegangen ist, aber insgesamt immer noch 576 Stellen unter Einbeziehung der Fachhochschulen umfasst. Die Chancen des wissenschaftlichen Nachwuchses auf eine Professur sind somit auch zumindest in den nächsten Jahren noch weiterhin relativ günstig, jedoch sollten bei der steigenden Anzahl an wissenschaftlichen Personal deutlich mehr Bewerbungen auf eine Professur kommen und somit zwangsläufig auch prozentual gesehen die Anzahl des wissenschaftlichen Personals, das nie eine Professur besetzen wird. Dabei ist zu erwarten, dass auch zukünftig der Hauptweg zu einer erziehungswissenschaftlichen Professur über das wissenschaftliche Personal und die Habilitation führen wird. Denn Juniorprofessuren wurden im Fach Erziehungswissenschaft in den Jahren 2002-2006 nur 53, in den Jahren 2007-2010 nur 46, in den Jahren 2010-2014 60 und in den Jahren 2014-2018 nur 44 ausgeschrieben, die sich zudem über zehn verschiedene Subdisziplinen verteilen. Bei den insgesamt 193 Ausschreibungen in einem Zeitraum von 16 Jahren muss es sich außerdem bereits häufig um Zweitausschreibungen oder um die erste bzw. zweite Wiederbesetzung von frei gewordenen Stellen handeln, da wie gezeigt in der amtlichen Statistik im Jahr 2018 nur 93 Juniorprofessuren im Fach Erziehungswissenschaft erfasst waren (vgl. Tabelle 4.2).

Abbildung 4.1: Ausgeschriebene Professuren in der Erziehungswissenschaft (ohne Fachdidaktik und ohne Juniorprofessuren) und Sozialpädagogik an den bundesdeutschen Hochschulen nach alten und neuen Bundesländern (1991-2018; $\mathrm{n}=4.127)$

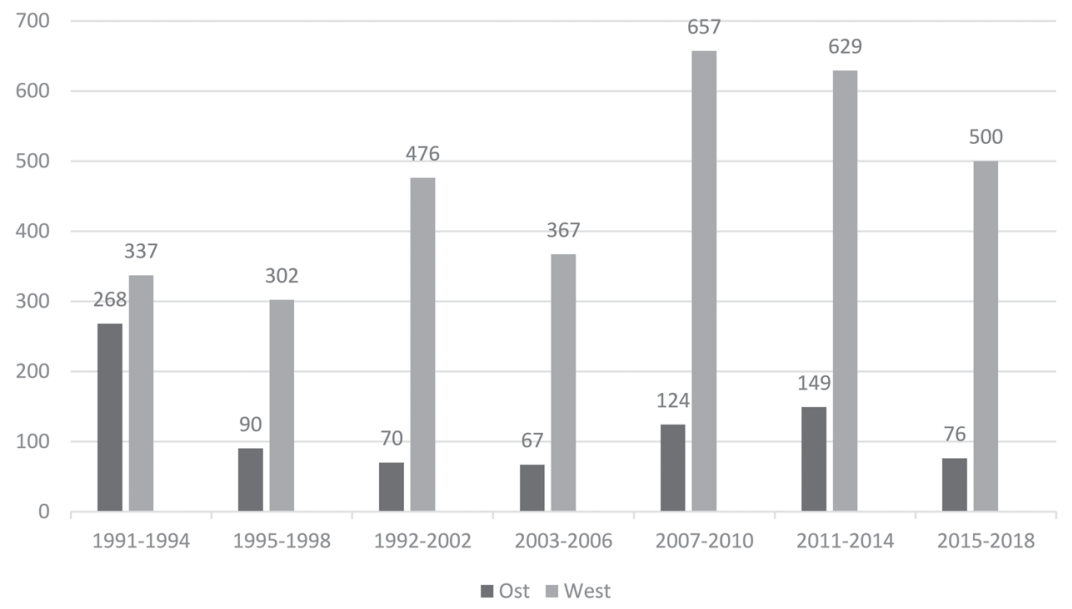

Quelle: Deutsche Universitätszeitung; Die Zeit; Rauschenbach/Christ 1994; Krüger/Weishaupt 2000; Krüger/Schmidt/Siebholz/Weishaupt 2004; Krüger/Schnoor/Weishaupt 2008; Krüger/ Kücker/Weishaupt 2012; Gerecht/Krüger/Post/Weishaupt 2016; eigene Berechnungen 
Den Ersatzbedarf an frei werdenden Hochschullehrerstellen im Fach Erziehungswissenschaft wird man auch im nächsten Jahrzehnt auf dem alternativen Weg zu einer Hochschullehrerlaufbahn über eine Juniorprofessur jedenfalls nicht ansatzweise abdecken können, wenn es nicht zu einer erheblichen Stellenausweitung kommt. Doch auch der traditionelle Weg könnte, wie die Entwicklung der Promovierenden (Vollzeitstellen als wissenschaftliches Personal) zeigt, zu einem Engpassfaktor für die Nachwuchsrekrutierung werden, wenn nicht umgehend das Stellenangebot für diese Phase der Hochschullaufbahn ausgebaut wird.

Dass weiterhin Ersatzbedarf in der Erziehungswissenschaft besteht, lässt Tabelle 4.15 erkennen. Er ist allerdings nicht mehr ganz so hoch, wie in den zurückliegenden Jahren. Bekanntlich gibt es mehrere Bundesländer, in denen die Dienstzeit verlängert werden kann oder gar muss. Hinzu kommt die 2012 einsetzende Hinausschiebung des Renten- bzw. Pensionszeitpunkts um einen Monat pro Jahr. Beide Entwicklungen verlängern die Beschäftigungsdauer, wirkten aber schon in der Vergangenheit oder machen sich nur langsam bemerkbar. Insofern können die Angaben in Tabelle 4.15 durchaus die Orientierung liefern, dass auch in den kommenden sieben bis acht Jahren etwa $38 \%$ der erziehungswissenschaftlichen Professuren neu besetzt werden müssen. Nicht zu erkennen ist eine Phase ohne nennenswerten Ersatzbedarf in der Erziehungswissenschaft insgesamt, was aber in einzelnen Subdisziplinen dennoch phasenweise zu Schließungstendenzen führen kann. Alles in allem ist zwar noch ein ausreichender Ersatzbedarf zu verzeichnen. Im Unterschied zum vergangenen Jahrzehnt werden jedoch die Wege in die Hochschullehrerlaufbahn im Fach Erziehungswissenschaft in den nächsten Jahren etwas schwieriger werden und die Anzahl an Personen, die nach alternativen Beschäftigungsmöglichkeiten suchen, steigen.

Tabelle 4.15: Professuren auf Dauerstellen in der Erziehungswissenschaft an wissenschaftlichen Hochschulen nach Besoldung und Jahr des voraussichtlichen Ausscheidens im Alter von 65 Jahren bis 2027/28

\begin{tabular}{|c|c|c|c|c|c|c|c|}
\hline & & $2019 / 20$ & $2021 / 22$ & $2023 / 24$ & $2025 / 26$ & $2027 / 28$ & in $\%^{1}$ \\
\hline \multirow{2}{*}{$\begin{array}{l}\text { Erziehungs- } \\
\text { wissenschaft }\end{array}$} & C4/W3 & 14 & 23 & 29 & 32 & 30 & 31,6 \\
\hline & $\mathrm{C} 3 / \mathrm{W} 2$ & 14 & 19 & 7 & 9 & 16 & 24,1 \\
\hline \multirow{2}{*}{$\begin{array}{l}\text { Sonder- } \\
\text { pädagogik }\end{array}$} & C4/W3 & 8 & 5 & 8 & 8 & 10 & 43,8 \\
\hline & C3/W2 & 1 & 4 & 3 & 6 & 4 & 32,7 \\
\hline \multirow{2}{*}{$\begin{array}{l}\text { Sozial- } \\
\text { pädagogik }\end{array}$} & C4/W3 & 3 & 2 & 2 & 1 & 4 & 50,0 \\
\hline & C3/W2 & 5 & 5 & 3 & 4 & 5 & 46,8 \\
\hline \multirow{2}{*}{ insgesamt } & C4/W3 & 25 & 30 & 39 & 41 & 45 & 34,8 \\
\hline & C3/W2 & 20 & 28 & 13 & 19 & 24 & 28,0 \\
\hline
\end{tabular}

1 aller Professuren der entsprechenden Besoldungsstufen 2017

Quelle: Statistisches Bundesamt, Sonderauswertungen der Hochschulpersonalstatistik, eigene Berechnungen 


\subsection{Geschlechterverhältnisse in den Erziehungswissenschaften}

In diesem Abschnitt wird das Verhältnis der Geschlechter in der Personalstruktur der Erziehungswissenschaft anhand der Professuren und des Mittelbaus beschrieben. Erziehungswissenschaften respektive Soziale Arbeit sind Studiengänge, die eher von Studentinnen präferiert werden, sodass etwa drei Viertel der Studierenden in diesen Fächern weiblichen Geschlechts sind.

Aus Tabelle 4.16 ist zu erkennen, dass der Anteil an Professorinnen in den Erziehungswissenschaften von 1995 kontinuierlich angestiegen ist. War zu diesem Zeitraum nur jede fünfte Professur durch eine Frau besetzt, kann für 2018 konstatiert werden, dass Geschlechterparität besteht. Kritisch eingeräumt werden muss hier jedoch, dass C4/W3-Stellen nach wie vor überwiegend durch Professoren besetzt sind (53,8 \% ohne Tabelle), während C3/W2-Professuren mit 54,6 \% und insbesondere C2/W1-Stellen mit 53,9\% vornehmlich weiblich besetzt sind. Inwiefern es sich hierbei um ein »Relikt« aus dem vorangegangen Jahren mit eher männlichen Stellenbesetzungen handelt, kann aus den vorliegenden Daten nicht geschlussfolgert werden.

Tabelle 4.16: Anteil der durch Frauen besetzten Professuren von 1995 bis $2018^{1}$

\begin{tabular}{|c|c|c|c|c|c|c|c|}
\hline \multicolumn{4}{|c|}{ Erziehungswissenschaften ${ }^{2}$} & \multirow{2}{*}{$\begin{array}{c}\text { Psychologie } \\
\text { weiblich } \\
\text { in } \%\end{array}$} & \multirow{2}{*}{$\begin{array}{l}\text { Politikwissen- } \\
\text { schaften } \\
\text { weiblich } \\
\text { in } \%\end{array}$} & \multirow{2}{*}{$\begin{array}{l}\text { Sozialwissen- } \\
\text { schaften } \\
\text { weiblich } \\
\text { in } \%\end{array}$} & \multirow{2}{*}{$\begin{array}{l}\text { Wirtschafts- } \\
\text { wissenschaften } \\
\text { weiblich } \\
\text { in } \%\end{array}$} \\
\hline Jahr & Professuren & $\begin{array}{c}\text { davon } \\
\text { weiblich }\end{array}$ & $\begin{array}{c}\text { weibl. } \\
\text { in } \%\end{array}$ & & & & \\
\hline 1995 & 1.091 & 211 & 19 & 15 & 11 & 11 & 4 \\
\hline 2000 & 979 & 238 & 24 & 17 & 13 & 15 & 5 \\
\hline 2005 & 861 & 264 & 31 & 22 & 18 & 22 & 7 \\
\hline 2010 & 934 & 397 & 43 & 32 & 26 & 29 & 13 \\
\hline 2014 & 1.011 & 456 & 45 & 37 & 30 & 38 & 17 \\
\hline 2015 & 1.021 & 471 & 46 & 39 & 29 & 39 & 18 \\
\hline 2016 & 1.038 & 494 & 48 & 38 & 30 & 39 & 19 \\
\hline 2017 & 1.043 & 520 & 50 & 38 & 31 & 40 & 19 \\
\hline 2018 & 1.045 & 525 & 50 & 39 & 32 & 40 & 19 \\
\hline
\end{tabular}

1 ab 2007 ohne Kunsthochschulen

2 einschließlich Sonderpädagogik und bis einschließlich 2006 Sozialpädagogik

Quelle: Statistisches Bundesamt, Hochschulstatistik, eigene Berechnungen

Im Vergleich zu den Nachbardisziplinen Psychologie, Politikwissenschaften, Sozialwissenschaften und Wirtschaftswissenschaften wird deutlich, dass es in den Erziehungswissenschaften deutlich besser gelungen ist, die Professuren auch mit weiblichen Nachwuchs zu besetzten. Zwar sind auch in diesen Disziplinen Zuwächse der Stellenanteile zu verzeichnen, die durch Professorinnen besetzt werden, jedoch auf niedrigerem Niveau und nur die Sozialwissenschaften können mit dem Aufwuchs weiblicher Professuren mithalten: Während 
der Zuwachs an weiblichen Professoren in den Erziehungswissenschaften $31 \%$ beträgt, sind es in der Psychologie $24 \%$, in den Politikwissenschaften $21 \%$, in den Sozialwissenschaften $29 \%$ und in den Wirtschaftswissenschaften nur $15 \%$. Insgesamt darf dies als eine positive Entwicklung in den Erziehungswissenschaften gewürdigt werden.

Die zukünftigen Professoreninnen und Professoren rekrutieren sich, wie oben dargelegt, aus dem wissenschaftlichen Nachwuchs. Deshalb wird nachfolgend ein Blick auf das Geschlechterverhältnis im Mittelbau gelegt und dieses mit dem Anteil der Professorinnen abgeglichen.

Ähnlich wie bei den Professuren ist auch im Mittelbau ein Anstieg von Wissenschaftlerinnen zu verzeichnen (vgl. Tabelle 4.17). Im Jahr 1995 war jede zweite Assistentenstelle weiblich besetzt, bei den wissenschaftlichen Mitarbeitern waren es $45 \%$ und bei den Lehrkräften für besondere Aufgaben $35 \%$. 22 Jahre später stellen Mitarbeiterinnen in allen benannten Berufsgruppen die deutliche Mehrheit. Während bei den kaum noch vorhandenen Assistentenstellen diese Entwicklung eher vorsichtig interpretiert werden sollte, zeigt sich jedoch beim wissenschaftlichen Personal die Tendenz, dass die Quote dieser sich kaum noch von dem Geschlechterverhältnis der Studierenden unterscheidet. Bei den Lehrkräften für besondere Aufgaben ist zwischen 2015 und 2017 ein leichter Rückgang zu verzeichnen - 2018 aber ein erneuter Anstieg. Hier scheint sich das Verhältnis zwischen weiblichen und männlichen Beschäftigten bei $60 \mathrm{zu} 40$ einzupendeln.

Tabelle 4.17: Anteil der Frauen im Mittelbau in der Erziehungswissenschaft von 1995 bis 2018 nach Stellen

\begin{tabular}{|c|c|c|c|c|c|c|c|c|c|}
\hline \multirow[b]{2}{*}{ Jahr } & \multicolumn{3}{|c|}{ Hochschulassistenten } & \multicolumn{3}{|c|}{ wissenschaftl. Mitarbeiter } & \multicolumn{3}{|c|}{$\begin{array}{l}\text { Lehrkräfte für besondere } \\
\text { Aufgaben }\end{array}$} \\
\hline & $\begin{array}{c}\text { insge- } \\
\text { samt }\end{array}$ & $\begin{array}{c}\text { davon } \\
\text { weiblich }\end{array}$ & $\begin{array}{l}\text { weiblich } \\
\text { in } \%\end{array}$ & $\begin{array}{l}\text { insge- } \\
\text { samt }\end{array}$ & $\begin{array}{c}\text { davon } \\
\text { weiblich }\end{array}$ & $\begin{array}{l}\text { weiblich } \\
\text { in } \%\end{array}$ & $\begin{array}{c}\text { insge- } \\
\text { samt }\end{array}$ & $\begin{array}{c}\text { davon } \\
\text { weiblich }\end{array}$ & $\begin{array}{l}\text { weiblich } \\
\text { in } \%\end{array}$ \\
\hline 1995 & 320 & 153 & 49 & 1.573 & 708 & 45 & 287 & 100 & 35 \\
\hline 2000 & 277 & 130 & 47 & 1.597 & 770 & 48 & 333 & 155 & 47 \\
\hline 2005 & 190 & 98 & 52 & 1.782 & 1.002 & 56 & 329 & 152 & 46 \\
\hline 2010 & 50 & 24 & 48 & 2.920 & 1.900 & 65 & 518 & 297 & 57 \\
\hline 2014 & 44 & 22 & 50 & 3.434 & 2.312 & 67 & 731 & 459 & 63 \\
\hline 2015 & 40 & 19 & 48 & 3.526 & 2.379 & 67 & 790 & 498 & 63 \\
\hline 2016 & 38 & 23 & 61 & 3.644 & 2.498 & 69 & 727 & 438 & 60 \\
\hline 2017 & 41 & 29 & 71 & 3.893 & 2.698 & 69 & 780 & 471 & 60 \\
\hline 2018 & 52 & 32 & 62 & 4.189 & 2.906 & 69 & 779 & 484 & 62 \\
\hline
\end{tabular}

Quelle: Statistisches Bundesamt, Hochschulstatistik, eigene Berechnungen

Resümierend zeigt die Entwicklung innerhalb der Erziehungswissenschaften, dass Geschlechterparität herstellbar ist, sofern der entsprechende Wille hierzu vorhanden ist. Sicherlich könnte auch diskutiert werden, ob zukünftig nicht das Verhältnis der Studierenden oder wissenschaftlichen Mitarbeiterstellen sich auch 
in dem Geschlechterverhältnis der Professorenschaft abbilden sollte. Die Grundlage hierfür ist durch fast $70 \%$ wissenschaftliche Mitarbeiterinnen gegeben.

\subsection{Fazit}

Insgesamt lässt sich festhalten, dass die Anzahl der Professuren in der Erziehungswissenschaft weiter angestiegen ist und sich nun fast wieder auf dem Ausgangsniveau von 1995 bewegt und im Jahre 2017 endlich die Geschlechterparität bei der Besetzung der Professuren erzielt wurde. Im Berichtsjahr 2018 sind erstmals mehr erziehungswissenschaftliche Professuren von Frauen als von Männern besetzt. Auch der Mittelbau expandiert weiter, insbesondere beim in der Forschung tätigen Personal. Dabei beruht der Aufwuchs in erster Linie auf dem Zuwachs von befristeten Vollzeit- und Teilzeitstellen in diesem Bereich, nur wenige Dauerstellen sind dort seit dem letzten Berichtszeitraum entstanden. Das in der Lehre tätige Personal verzeichnet bei den hauptberuflichen Stellen nur minimale Zuwächse, das nebenberufliche Personal in diesem Bereich schrumpft sogar deutlich.

$\mathrm{Ob}$ die geringen hauptberuflichen Aufwüchse ausreichen, um die nebenberuflichen Verluste zu kompensieren, ist derzeit noch nicht absehbar. Vermutlich werden einige wenige attraktive nebenberufliche Stellen in hauptberufliche Stellen umgewandelt. Doch auch diese hauptberuflichen Stellen sind aufgrund des hohen Lehrdeputats und der damit fehlenden Zeit für eine eigene Weiterqualifizierung nicht sonderlich attraktiv. Zudem sind mehr als zwei Drittel der Stellen für Lehrkräfte mit besonderen Aufgaben befristet, 61 \% der befristeten Stellen laufen auf Teilzeit. Diese Zahlen sprechen nicht für eine nachhaltige und qualitätsorientierte Absicherung universitärer Lehre bei steigenden Studierendenzahlen.

Hinsichtlich des Stellenmarktes für Professuren kann auch in den kommenden acht Jahren weiterhin mit einem hohen Bedarf gerechnet werden, auch wenn die Stellenausschreibungen in den vergangenen vier Jahren rückläufig waren. Immer noch sind rund ein Drittel der Professuren in den kommenden beiden Berichtszeiträumen neu zu besetzen. Dennoch sind die Chancen, eine eigene Professur zu bekommen, leicht zurückgegangen. Wie im vergangenen Berichtszeitraum sind die geringen Stellen- und Ausschreibungsvolumina der Juniorprofessuren anzumahnen, die in Anbetracht des sich abzeichnenden Bedarfs deutlich gestärkt werden müssten. Dies würde gleichzeitig zu einem Ausbau der Lehrkapazitäten führen - die leider nicht vom neuerlichen Personalaufwuchs im Mittelbau profitieren konnten - und damit zur Absicherung qualitativ hochwertiger Lehre beitragen.

In den Subdisziplinen der Erziehungswissenschaften ist die Lage der Stellenausschreibungen relativ stabil geblieben, wobei zu beobachten ist, dass für den Bereich Schulpädagogik vermehrt Professuren für die empirische Bil- 
dungsforschung bzw. für die empirische Schul- und Unterrichtsforschung ausgeschrieben werden. Erstmals nehmen diese Stellen mit einem Anteil von 22 \% am Stellenausschreibungsvolumen einen Spitzenplatz ein. Ob dadurch die Forschungsorientierung im Fach Erziehungswissenschaft deutlich verbessert werden konnte, werden die empirischen Analysen in den zukünftig zu erstellenden Datenreports noch zeigen.

\section{Literatur}

Autorengruppe Kinder- und Jugendhilfestatistik (Hrsg.) (2019): Kinder- und Jugendhilfereport 2018: Eine kennzahlenbasierte Analyse. Opladen u.a.: Verlag Barbara Budrich.

Baumert, Jürgen/Roeder, Peter Martin (1994): »Stille Revolution«. Zur empirischen Lage der Erziehungswissenschaft. In: Krüger, H.-H./Rauschenbach, T. (Hrsg.): Erziehungswissenschaft. Weinheim: Juventa, S. 29-48.

Krüger, Heinz-Hermann/Weishaupt, Horst (2000): Personal. In: DGfE: Datenreport Erziehungswissenschaft. Befunde und Materialien zur Lage und Entwicklung des Faches in der Bundesrepublik. Hrsg. v. Otto, H.-U./Krüger, H.-H./Merkens, H./ Rauschenbach, T./Schenk, B./Weishaupt, H./Zedler, P. Schriften der Deutschen Gesellschaft für Erziehungswissenschaft. Opladen: Leske + Budrich, S. 75-98.

Krüger, Heinz-Hermann/Schmidt, Claudia/Siebholz, Susanne/Weishaupt, Horst (2004): Personal. In: DGfE: Datenreport Erziehungswissenschaft 2004. Hrsg. v. Tippelt, R./ Rauschenbach, T./Weishaupt, H. Schriften der Deutschen Erziehungswissenschaft für Erziehungswissenschaft. Wiesbaden: VS Verlag für Sozialwissenschaften, S. 63-89.

Krüger, Heinz-Hermann/Schnoor, Oliver/Weishaupt, Horst (2008): Personal. In: DGfE: Datenreport Erziehungswissenschaft 2008. Hrsg. v. Tillmann, K.-J./Rauschenbach, T./Tippelt, R./Weishaupt, H. Schriften der Deutschen Gesellschaft für Erziehungswissenschaft. Opladen u.a.: Verlag Barbara Budrich, S. 87-112.

Krüger, Heinz-Hermann/Kücker, Caroline/Weishaupt, Horst (2012): Personal. In: DGfE: Datenreport Erziehungswissenschaft 2012. Hrsg. v. Thole, W./Faulstich-Wieland, H./Horn, K.-P./Weishaupt, H./Züchner, I. Schriften der Deutschen Gesellschaft für Erziehungswissenschaft. Opladen u.a.: Verlag Barbara Budrich, S. 137-158.

Gerecht, Marius/Krüger, Heinz-Hermann/Post, Andreas/Weishaupt, Horst (2016): Personal. In: DGfE: Datenreport Erziehungswissenschaft 2016. Hrsg. v. Koller, H.-C./ Faulstich-Wieland, H./Weishaupt, H./Züchner, I. Schriften der Deutschen Gesellschaft für Erziehungswissenschaft. Opladen u.a.: Verlag Barbara Budrich. Opladen: Verlag Barbara Budrich, S. 135-158.

Rauschenbach, Thomas/Christ, Bettina. (1994): Abbau, Wandel der Expansion? Zur disziplinären Entwicklung der Erziehungswissenschaft im Spiegel ihrer Stellenbesetzungen. In: Krüger, H.-H./Rauschenbach, T. (Hrsg.): Erziehungswissenschaft. Weinheim: Juventa, S. 69-92. 



\title{
5 Forschung und Publikationskulturen
}

\author{
Bernhard Schmidt-Hertha \& Margaretha Müller
}

Das Thema Forschung und Publikationskulturen ist seit der ersten Ausgabe im Jahr 2000 fester Bestandteil des Datenreports. Dabei geht es nicht um eine Bewertung von Forschungs- und Publikationsstrategien - soweit hier überhaupt von strategischen Erwägungen ausgegangen werden kann -, sondern um die Beschreibung der Veränderungen im Wissenschaftsbetrieb aus Sicht einer Disziplin und im Vergleich mit ihren Nachbardisziplinen. Es soll hier weder einer immer stärker auf Projektförderung ausgerichteten Wissenschaftspolitik mit all ihren Vor- und Nachteilen (vgl. Torka 2018) das Wort geredet werden, noch bestimmte Publikationsformate über andere erhoben werden. Im Zentrum steht die Dokumentation von Entwicklungen anhand öffentlicher Statistik und anderen für wissenschaftliche Analysen zur Verfügung stehenden Daten.

Drittmittel und Publikationen sind zwar zunächst unabhängig voneinander zu betrachten, scheinen aber empirisch durchaus in Verbindung zu stehen (vgl. Gralka/Wohlrabe/Bornmann 2018). Es ist zum einen davon auszugehen, dass Qualität und Quantität von Veröffentlichungen einen Einfluss auf die Erfolgschancen bei kompetitiven Verfahren der Drittmittelvergabe haben. Zum anderen ist zu erwarten, dass Drittmittel mit einer Intensivierung von Forschungsleistungen (und damit publizierbaren Ergebnissen) verbunden sowie mit der Erwartungshaltung verknüpft sein können, Projektergebnisse sichtbar zu veröffentlichen. Gleichzeitig sind beide Bereiche - Drittmittel und Publikationen - auch an institutionelle Rahmungen gebunden (z.B. Christensen/Dyrstad/Innstrand 2018) und es sind entsprechend sowohl auf Ebene einzelner Hochschulen als auch auf Bundeslandebene Intergruppendifferenzen (z.B. in Abhängigkeit von hochschulpolitischen Rahmungen auf Landesebene, regionaler Forschungsförderung, Hochschulform etc.) zu erwarten. Inwieweit Forschungsintensität dabei in Konkurrenz zu Engagement in der universitären Lehre tritt oder beide Bereiche sich gegenseitig befruchten, kann hier nicht weiter untersucht werden. Vorliegende Studien deuten allerdings daraufhin, dass der Zusammenhang zumindest deutlich komplexer ist (Enders/Teichler 1997) und es einzelnen Standorten gelingt in beiden Bereichen - Forschung und Lehre - gleichermaßen erfolgreich zu sein, während andere nur in einem oder keinem der beiden zentralen universitären Aufgabenfelder Überdurchschnittliches leisten (vgl. Schmidt-Hertha/ Tafertshofer/Tippelt 2016). 


\subsection{Forschungsförderung und Drittmittel}

Zunächst ist festzustellen, dass die Informationen aus der öffentlichen Statistik zur Forschungsförderung zwar kontinuierlich fortgeschrieben werden konnten, aber angesichts der zunehmenden wissenschaftspolitischen Bedeutung von Drittmitteln die öffentlichen Statistiken längst keine hinreichende Differenzierung mehr für eine systematische Dokumentation von Ist-Ständen und Veränderungen in der Forschungslandschaft bieten. Die hierfür anschlussfähigen weiteren Datenquellen sind hilfreich, aber in vielerlei Hinsicht ebenso unbefriedigend. Während die DFG über ihren Förderatlas zumindest die über diese zentrale Fördereinrichtung fließenden Drittmittelströme dokumentiert, liegen für andere wichtige Drittmittelgeber (insbesondere Bundes- und Landesministerien sowie Stiftungen) keine vergleichbaren Daten vor. In vorangehenden Ausgaben des Datenreports wurde daher u.a. auf die vom CHE gesammelten Daten und die in der Datenbank SOFIS hinterlegten Informationen zu Forschungsprojekten zurückgegriffen. Beides ist im Folgenden nicht bzw. nur sehr eingeschränkt möglich. Während wir auf die Nutzung von CHE-Daten insbesondere aufgrund anhaltender kritischer Diskurse um deren Qualität und Aussagekraft (vgl. Deutsche Gesellschaft für Soziologie 2012) in diesem wie auch im letzten Datenreport verzichtet haben, wurde die Datenbank SOFIS inzwischen eingestellt. Bis 2017 wurden zwar noch neue Projekte in der Datenbank erfasst, die Daten zeigen aber deutlich, dass dies nicht mehr mit gleicher Intensität und Nachhaltigkeit gepflegt wurde wie in vorangegangenen Jahren.

Insgesamt ist es überraschend, wie defizitär die verfügbaren Informationen $\mathrm{zu}$ so zentralen Forschungsindikatoren sind und wie intransparent dadurch auch Finanzierungswege und Drittmittelströme nicht nur im Feld der Erziehungswissenschaft werden. Mit Wegfall der SOFIS-Datenbank lassen sich darüber hinaus kaum noch Aussagen zu inhaltlichen Schwerpunkten in der erziehungswissenschaftlichen Forschung auf breiter Datenbasis machen.

\subsubsection{Drittmittelvolumen}

$\mathrm{Zu}$ Beginn werden die seit 1995 dokumentierten Drittmitteleinnahmen im Fach Erziehungswissenschaft und den Vergleichsdisziplinen fortgesetzt und im Anschluss auf das aus diesen Geldern finanzierte hauptberufliche Personal eingegangen. Die Daten hierfür stammen vom Statistischen Bundesamt und beziehen sich auf die wissenschaftlichen Hochschulen. ${ }^{1}$ Bei den Angaben pro Professur handelt es sich jeweils um die entsprechenden Professuren vor Ort und nicht allein um die antragstellenden Professorinnen und Professoren.

1 Wir danken Frau Brigitte Damm und Herrn Thomas Weise vom Statistischen Bundesamt für die sehr schnelle und unbürokratische Bereitstellung der Sonderauswertungen. 
Tabelle 5.1: Drittmitteleinnahmen der wissenschaftlichen Hochschulen (ohne Fachhochschulen) nach ausgewählten Lehr- und Forschungsbereichen 1995, 2000, 2005, 2010-2017, in Tausend Euro

\begin{tabular}{|c|c|c|c|c|c|}
\hline Jahr & $\begin{array}{l}\text { Erziehungs- } \\
\text { wissenschaft }^{1}\end{array}$ & Psychologie & $\begin{array}{l}\text { Politik- } \\
\text { wissenschaft }\end{array}$ & $\begin{array}{l}\text { Sozial- } \\
\text { wissenschaft }\end{array}$ & $\begin{array}{l}\text { Wirtschafts- } \\
\text { wissenschaft }\end{array}$ \\
\hline 1995 & 21.295 & 19.002 & 12.842 & 12.945 & 30.657 \\
\hline 2000 & 28.551 & 28.477 & 11.926 & 21.221 & 61.151 \\
\hline 2005 & 45.418 & 41.345 & 19.353 & 23.410 & 89.621 \\
\hline 2010 & 61.309 & 62.400 & 22.228 & 39.573 & 124.897 \\
\hline 2011 & 64.298 & 68.339 & 24.293 & 48.063 & 131.022 \\
\hline 2012 & 74.728 & 68.090 & 25.161 & 49.747 & 138.377 \\
\hline 2013 & 80.523 & 76.452 & 30.130 & 51.723 & 143.289 \\
\hline 2014 & 82.493 & 75.158 & 32.353 & 52.847 & 148.133 \\
\hline 2015 & 72.494 & 80.766 & 30.107 & 55.529 & 141.492 \\
\hline 2016 & 91.927 & 77.673 & 29.435 & 50.254 & 148.998 \\
\hline 2017 & 98.363 & 86.453 & 29.721 & 56.807 & 157.120 \\
\hline \multicolumn{6}{|c|}{ Drittmitteleinnahmen je Professur (in Tausend Euro) } \\
\hline 1995 & 18,9 & 35,8 & 47,0 & 25,1 & 23,0 \\
\hline 2000 & 31,5 & 54,3 & 44,0 & 44,7 & 45,1 \\
\hline 2005 & 53,1 & 71,7 & 62,4 & 53,8 & 64,1 \\
\hline 2010 & $72,5^{2}$ & 108,1 & 70,1 & 86,2 & 73,5 \\
\hline 2011 & 68,8 & 118,9 & 74,1 & 104,0 & 72,7 \\
\hline 2012 & 79,2 & 113,5 & 75,8 & 108,4 & 73,6 \\
\hline 2013 & 82,5 & 122,0 & 86,1 & 108,2 & 71,3 \\
\hline 2014 & 82,6 & 116,0 & 91,7 & 108,3 & 72,9 \\
\hline 2015 & 72,3 & 122,0 & 85,0 & 108,9 & 67,7 \\
\hline 2016 & 90,4 & 111,8 & 80,9 & 99,3 & 71,2 \\
\hline 2017 & $96,7^{3}$ & 123,3 & 81,2 & 110,5 & 72,4 \\
\hline
\end{tabular}

1 einschließlich Sonder- und Sozialpädagogik

2 ohne Professuren für Sozialpädagogik

3 Anzahl Professuren 2016

Quelle: Statistisches Bundesamt, Fachserie 11, Reihe 4.4 und 4.5; eigene Berechnungen 
Der bisherige Trend der kontinuierlichen Steigerung des Drittmittelaufkommens in den Fächern seit 1995 kann trotz einiger Schwankungen ab 2014 weiter beobachtet werden. Ausnahme bildet hier lediglich die Politikwissenschaft, für die eher eine Stagnation im Drittmittelvolumen eingetreten zu sein scheint. In der Erziehungswissenschaft ist - nach einem vorübergehenden Rückgang der Drittmittel im Jahr 2015 - ein sprunghafter Zuwachs in 2016 auffällig. Hier könnten große Förderprogramme und insbesondere die Qualitätsoffensive Lehrerbildung des BMBF eine wesentliche Rolle gespielt haben. Die im Auswahlverfahren erfolgreichen Projekte, die an den jeweiligen Standorten oft in erheblichem Maße in der Erziehungswissenschaft verankert waren und sind, konnten zum Teil schon 2015 beginnen, das Gros der Mittel wurde aber erst 2016 und in den Folgejahren abgerufen.

Ein etwas verändertes Bild ergibt sich bezogen auf die Drittmittel pro Professur. Hier zeigt sich insbesondere in den Erziehungswissenschaften eine deutliche Erhöhung der Drittmittel seit 2014. Da für 2017 lediglich die Anzahl der Professuren ohne Sozialpädagogik vorlag, wurde hier zur besseren Annäherung auf die Zahl von Professuren von 2016 zurückgegriffen. Ebenfalls in der Psychologie kann eine Steigerung festgestellt werden, während die anderen Fächer eher stagnieren bzw. die Drittmittel pro Professur sogar abnehmen. Auch wenn die Professorinnen und Professoren in der Erziehungswissenschaft mit durchschnittlich 96.700 Euro im Jahr an Drittmitteln aufgeholt haben, können sie nach wie vor auf weniger Forschungsgelder zugreifen als Kolleginnen und Kollegen in der Psychologie und den Sozialwissenschaften.

Die seit 1995 parallel zum erworbenen Drittmittelvolumen vorhandene Steigerung der Anzahl der aus diesen Geldern finanzierten Stellen bleibt mit einem Einbruch im Jahr 2014 - ebenfalls vorhanden (vgl. Tabelle 5.2.). Dies gilt, mit Ausnahme der Wirtschaftswissenschaften, für alle Fächer. Bei Betrachtung des hauptamtlichen Personals aus Drittmitteln pro Professur zeigt sich für die Erziehungswissenschaft seit der Stagnation beim letzten Datenreport wieder eine Erhöhung seit dem Jahr 2016. Die Erziehungswissenschaft kann nun mit 1,49 Mitarbeitenden pro Professur fast mit den Sozialwissenschaften mithalten, erreicht aber auch in diesem Indikator nicht die Forschungsstärke der Psychologie $(2,26)$. 
Tabelle 5.2: Aus öffentlichen und privaten Drittmitteln finanziertes hauptberufliches wissenschaftliches und künstlerisches Personal an wissenschaftlichen Hochschulen (ohne Fachhochschulen) 1995, 2000, 2005 und 2010-2018

\begin{tabular}{|c|c|c|c|c|c|}
\hline Jahr & $\begin{array}{l}\text { Erziehungs- } \\
\text { wissenschaft' }\end{array}$ & Psychologie & $\begin{array}{l}\text { Politik- } \\
\text { wissenschaft }\end{array}$ & $\begin{array}{l}\text { Sozial- } \\
\text { wissenschaft }\end{array}$ & $\begin{array}{l}\text { Wirtschafts- } \\
\text { wissenschaft }\end{array}$ \\
\hline $1995^{3}$ & 302 & 419 & $136^{2}$ & $271^{2}$ & 571 \\
\hline $2000^{3}$ & 370 & 529 & 186 & 348 & 908 \\
\hline 2005 & 456 & 658 & 176 & 302 & 941 \\
\hline 2010 & 1.064 & 1.178 & 244 & 587 & 1.948 \\
\hline 2011 & 1.116 & 1.291 & 283 & 677 & 1.965 \\
\hline 2012 & 1.179 & 1.352 & 320 & 707 & 2.129 \\
\hline 2013 & 1.240 & 1.332 & 381 & 722 & 2.230 \\
\hline 2014 & 1.178 & 1.297 & 364 & 716 & 2.134 \\
\hline 2015 & 1.207 & 1.375 & 388 & 740 & 1.917 \\
\hline 2016 & 1.430 & 1.543 & 435 & 800 & 2.094 \\
\hline 2017 & 1.572 & 1.556 & 456 & 843 & 2.209 \\
\hline 2018 & 1.559 & 1.612 & 479 & 866 & 2.085 \\
\hline \multicolumn{6}{|c|}{ hauptberufliche Projektmitarbeiter aus Drittmitteln je Professur } \\
\hline $1995^{3}$ & 0,29 & 0,79 & $(0,50)$ & $(0,53)$ & 0,43 \\
\hline $2000^{3}$ & 0,41 & 1,01 & 0,68 & 0,73 & 0,67 \\
\hline 2005 & 0,53 & 1,14 & 0,57 & 0,69 & 0,67 \\
\hline 2010 & $1,26^{4}$ & 2,04 & 0,77 & 1,28 & 1,15 \\
\hline 2011 & 1,19 & 2,25 & 0,86 & 1,47 & 1,09 \\
\hline 2012 & 1,25 & 2,25 & 0,96 & 1,54 & 1,13 \\
\hline 2013 & 1,27 & 2,13 & 1,09 & 1,51 & 1,11 \\
\hline 2014 & 1,18 & 2,00 & 1,03 & 1,47 & 1,05 \\
\hline 2015 & 1,20 & 2,08 & 1,10 & 1,45 & 0,92 \\
\hline 2016 & 1,41 & 2,22 & 1,20 & 1,58 & 1,00 \\
\hline 2017 & $1,55^{5}$ & 2,22 & 1,25 & 1,64 & 1,02 \\
\hline 2018 & 1,49 & 2,26 & 1,30 & 1,66 & 0,95 \\
\hline
\end{tabular}

1 einschließlich Sonder- und Sozialpädagogik

2 Aufteilung geschätzt

3 einschließlich Fachhochschulen, Erziehungswissenschaft ohne Sozialpädagogik

4 ohne Professuren für Sozialpädagogik

5 Anzahl Professuren 2016

Quelle: Statistisches Bundesamt: Fachserie 11, Reihe 4.4; unveröffentlichtes Material des Statistischen Bundesamts; eigene Berechnungen 


\subsubsection{Arten und Finanzierung von Forschungsprojekten}

Neben dem Drittmittelvolumen sind Finanzierungsformen (Forschungsförderung vs. Auftragsforschung) und die Quellen der Forschungsfinanzierung für die Ausrichtung und Einordnung von Forschung bedeutend (vgl. Dilger 2018). Zur Identifizierung der Quellen eingeworbener Drittmittel, die in der amtlichen Statistik kaum differenziert werden, wurde bisher auf die Forschungsdatenbank SOFIS zurückgegriffen. Dieses Angebot des ehemaligen Informationszentrums Sozialwissenschaften (IZ) der Gesellschaft Sozialwissenschaftlicher Infrastruktureinrichtungen (GESIS) wurde jedoch Ende 2017 eingestellt. SOFIS stellte die wichtigste und umfangreichste Sammlung erziehungswissenschaftlicher Forschungsprojekte dar. Diese ergab sich einerseits anhand der Meldung geförderter Projekte durch wichtige Drittmittelgeber wie z.B. die DFG und andererseits wurden die einzelnen Fachbereiche und Institute in regelmäßigen Abständen gebeten, ihre aktuellen Forschungsprojekte zu melden. Zusätzlich zu den Drittmittelprojekten umfasste die Datenbank auch aus Eigenmitteln finanzierte Forschungsprojekte sowie nicht geförderte Qualifikationsarbeiten. Durch die Möglichkeit der Mehrfachzuordnungen waren viele Projekte hierbei mehreren Kategorien (z.B. Auftragsforschung und Qualifikationsarbeiten) zugewiesen.

Das Resultat war ein nahezu vollständiges und vermutlich repräsentatives Verzeichnis finanziell geförderter Forschungsprojekte der Erziehungswissenschaft in Deutschland. Bisher existiert kein vergleichbares Angebot, was als problematisch zu betrachten ist. Für den Report liegen zwar noch Daten für die Jahre 2015-2017 ${ }^{2}$ vor, diese sind jedoch keinesfalls vollständig und nicht annähernd repräsentativ. Im Jahr 2017 wurden durch die geplante Einstellung der Datenbank am Ende des Jahres kaum noch Projekte aufgenommen. Aber auch schon im Jahr zuvor ist von einer reduzierten Aufnahme auszugehen, da die Akquise weit vor der Einstellung des SOFIS-Angebots stark zurückgefahren wurde. Die folgenden Daten sind daher mit Vorsicht zu interpretieren und nicht mit den Daten des letzten Reports vergleichbar. Für den Zeitraum 2015-2017 waren noch 751 erziehungs- und bildungswissenschaftliche Projekte gemeldet, bei 745 liegen Angaben zur Finanzierung vor. Rund zwei Drittel der Projekte wurden von Drittmitteln finanziert (67,7\%), wovon wiederum etwas weniger als ein Viertel der Auftragsforschung (19,2\%) und mehr als zwei Drittel der freien Forschungsförderung (70,8 \%) entfallen. 9,9\% haben sich beiden Finanzierungsformen zugeordnet. Mit Qualifikationsarbeiten ist ein Viertel aller verzeichneten Projekte verbunden (23,5\%). In SOFIS sind neben Projekten, die an den Universitäten angesiedelt sind, auch Forschungsaktivitäten anderer Hochschulen und außeruniversitärer Forschungseinrichtungen gemeldet. Dabei werden 28,9 \% der Projekte kooperativ von mindestens zwei Forschungseinrichtungen verantwortet.

2 Die Autoren danken dem GESIS-Leibniz-Institut für Sozialwissenschaften und insbesondere Herrn Jakowatz für die Beratung und die Bereitstellung der Daten. 
Bei Betrachtung der Verteilung der unterschiedlichen Geldgeber haben sich das BMBF (33\%), die DFG (20 \%), Stiftungen (15\%) als auch Landesministerien und -einrichtungen (14\%) als wichtigste Drittmittelquellen erwiesen. Für den starken Stellenwert des BMBF könnte die bereits erwähnte Qualitätsoffensive Lehrerbildung eine Rolle spielen. Der geringere Anteil an DFG geförderten Projekten steht möglicherweise im Zusammenhang mit der reduzierten Anzahl an DFG-Bewilligungen (siehe Tabelle 5.3). Dennoch ist - wie bereits erwähnt - auf die bedingte Aussagekraft der Daten hinzuweisen.

\subsubsection{DFG-Förderung}

Anders als in den Daten des Statistischen Bundesamts berichtet die DFG (2018) in ihrem Förderatlas, der als Datengrundlage für die folgenden Analysen dient, immer über einen Drei-Jahres-Zeitraum. Bislang liegen daher nur die Daten bis einschließlich 2016 vor. Die vergebenen Mittel werden im Förderatlas immer auf 100.000 Euro gerundet, weshalb Standorte mit einem Aufkommen von DFG-Mitteln unter 50.000 Euro im genannten Zeitraum in der Statistik der DFG mit 0 Euro geführt werden. In der Förderlogik der DFG werden überdies die Mittel immer demjenigen Fach zugeordnet, dem der Antrag selbst zugeordnet wurde, d.h. auch bei Verbundanträgen ist es möglich, dass auch die Mittel beteiligter Antragstellender aus anderen Fachbereichen der Erziehungswissenschaft zugeschlagen werden und umgekehrt erziehungswissenschaftliche Teilprojekte nicht in der hier herangezogenen Statistik berücksichtigt werden.

Tabelle 5.3: Anteil der DFG-Bewilligungen an Drittmitteleinnahmen der wissenschaftlichen Hochschulen insgesamt (in Mio. Euro; 2011-2013 und 2014-2016)

\begin{tabular}{|c|c|c|c|c|c|c|c|c|}
\hline & \multicolumn{2}{|c|}{$\begin{array}{l}\text { Erziehungs- } \\
\text { wissenschaft }\end{array}$} & \multicolumn{2}{|c|}{ Psychologie } & \multicolumn{2}{|c|}{$\begin{array}{l}\text { Sozial- } \\
\text { wissenschaften } \\
\text { (inkl. Politik- } \\
\text { wissenschaften) }\end{array}$} & \multicolumn{2}{|c|}{$\begin{array}{l}\text { Wirtschafts- } \\
\text { wissenschaften }\end{array}$} \\
\hline & $\begin{array}{l}2011- \\
2013\end{array}$ & $\begin{array}{l}2014- \\
2016\end{array}$ & $\begin{array}{l}2011- \\
2013\end{array}$ & $\begin{array}{l}2014- \\
2016\end{array}$ & $\begin{array}{l}2011- \\
2013\end{array}$ & $\begin{array}{l}2014- \\
2016\end{array}$ & $\begin{array}{l}2011- \\
2013\end{array}$ & $\begin{array}{l}2014- \\
2016\end{array}$ \\
\hline $\begin{array}{l}\text { Drittmitteleinnahmen } \\
\text { insgesamt }\end{array}$ & 219,5 & 246,9 & 221,9 & 233,6 & 229,1 & 250,5 & 412,7 & 438,6 \\
\hline DFG-Bewilligungen & 41,9 & 33,7 & 120,9 & 135,1 & $138,3^{1}$ & $170,5^{1}$ & 80,4 & 100,1 \\
\hline $\begin{array}{l}\text { Anteil DFG-Bewilligungen } \\
\text { an Drittmittel gesamt in \% }\end{array}$ & 19,1 & 13,6 & 54,5 & 57,8 & 60,4 & 68,1 & 19,5 & 22,8 \\
\hline $\begin{array}{l}\text { DFG-Bewilligungen pro } \\
\text { Professur }\end{array}$ & 0,044 & 0,033 & 0,202 & 0,202 & 0,172 & 0,397 & 0,044 & 0,048 \\
\hline
\end{tabular}

1 einschließlich Publizistik und Kommunikationswissenschaft

Quelle: Statistisches Bundesamt, Fachserie 11, Reihe 4.4 und 4.5; DFG-Förderatlas 2015 und 2018; eigene Berechnungen 
Wie im Zeitraum von 2011-2013 zeigt sich auch diesmal wieder eine ähnliche Summenhöhe der Drittmitteleinnahmen in der Erziehungswissenschaft, Psychologie sowie Sozial- und Politikwissenschaft, die sich unabhängig von der Größe des Faches darstellt. Dies gilt jedoch nicht für die Anteile der DFGBewilligungen an der Gesamtdrittmitteleinnahme. In allen Fächern, außer in den Erziehungswissenschaften, in der ein Rückgang zu verzeichnen ist, haben die DFG-Bewilligungen zum letzten Drei-Jahres-Zeitraum zugenommen. Der bereits vorhandene Unterschied der Erziehungswissenschaft insbesondere im Vergleich zur Psychologie und den Sozialwissenschaften wurde somit nochmals verschärft. Der Erziehungswissenschaft gelingt es demnach immer weniger, an den Mitteln der DFG zu partizipieren. Mit Blick auf die bewilligten DFG-Mittel pro Professur verstärkt sich die Differenz zu den Vergleichsdisziplinen weiter. Professorinnen und Professoren in den Erziehungswissenschaften verfügten 2014 bis 2016 mit durchschnittlich 33.000 Euro pro Professur im Drei-Jahres-Zeitraum über ein Sechstel der DFG-Mittel, die ihren Kolleginnen und Kollegen aus der Psychologie zur Verfügung standen. Im Vergleich mit den Sozialwissenschaften verdoppelt sich der Unterschied noch einmal. Auch in der Wirtschaftswissenschaft, die im letzten Zeitraum noch die gleiche Summe an DFG-Bewilligungen pro Professur aufwies wie die Erziehungswissenschaft, liegen die DFG-Mittel nun eineinhalbmal so hoch.

Es kann hier zwar argumentiert werden, dass gerade für stärker auf ein Anwendungsfeld bezogene Disziplinen - wie die Erziehungs- oder Wirtschaftswissenschaft - traditionell andere Forschungsförderer (insbesondere Bundesund Landesministerien, Stiftungen, die Europäische Union und Unternehmen) eine größere Rolle spielen und hier der relative Anteil von DFG-Mitteln traditionell geringer ist. Dass diese Mittel aber im interdisziplinären Vergleich so deutlich hinter jenen anderer sozialwissenschaftlicher Disziplinen zurückbleiben und in den vergangenen Jahren offensichtlich weiter gesunken sind, wirft Fragen auf. Ob der von Seiten der DFG oft genannte Mangel an qualitativ hochwertigen Anträgen oder die Tatsache, dass es der Erziehungswissenschaft nicht gelungen ist, in nennenswertem Umfang von den Mitteln aus der ExzellenzInitiative der DFG zu profitieren, ${ }^{3}$ die rückläufigen Fördermittel hinreichend zu erklären vermögen, kann hier nicht geklärt werden.

Erhebliche Schwankungen hinsichtlich der eingeworbenen DFG-Mittel zeigen sich für die Zeiträume 2011-2013 und 2014-2016 auf Ebene einzelner Hochschulen. Gerade für die besonders erfolgreich DFG-Mittel einwerbenden Standorte sind sehr starke Zuwächse oder Reduzierungen festzustellen, was u.a. mit größeren Verbundprojekten (insbesondere Graduiertenkollegs und Forschergruppen) in Zusammenhang stehen könnte.

3 In der Exzellenzrunde von 2013-2019 waren erziehungswissenschaftliche Fachbereiche lediglich an einer Graduiertenschule und an keinem Exzellenz-Cluster beteiligt. 
Tabelle 5.4: DFG-Bewilligungen nach Standorten (2011-2013 und 2014-2016)

\begin{tabular}{|c|c|c|c|c|}
\hline \multirow[t]{2}{*}{ Hochschule } & \multicolumn{2}{|c|}{$\begin{array}{l}\text { bewilligte Mittel } \\
\text { in Tausend Euro }\end{array}$} & \multicolumn{2}{|c|}{$\begin{array}{l}\text { Bewilligungen pro Professur } \\
\text { in Tausend Euro }\end{array}$} \\
\hline & 2011-2013 & 2014-2016 & 2011-2013 & 2014-2016 \\
\hline Augsburg U & 200 & 200 & 27 & 25 \\
\hline Bamberg U & 4.700 & 2.100 & 588 & 191 \\
\hline Berlin FU & 1.800 & 400 & 109 & 19 \\
\hline Berlin HU & 2.000 & 1.200 & 70 & 44 \\
\hline Berlin TU & 300 & 100 & 60 & 25 \\
\hline Bielefeld U & 300 & 400 & 19 & 22 \\
\hline Bochum U & 700 & 300 & 70 & 25 \\
\hline Braunschweig TU* & 100 & - & 13 & - \\
\hline Bremen JU & 300 & 400 & 300 & k. A. \\
\hline Bremen U & 200 & 100 & 13 & 7 \\
\hline Darmstadt TU*** & 300 & 0 & 43 & 0 \\
\hline Dortmund TU & 1.600 & 1.100 & 59 & 38 \\
\hline Dresden TU & 700 & 600 & 50 & 27 \\
\hline Duisburg-Essen U & 2.900 & 1.900 & 135 & 90 \\
\hline $\begin{array}{l}\text { Eichstätt-Ingolstadt } \\
\text { KathU* }^{*}\end{array}$ & 100 & - & 20 & - \\
\hline Erfurt U & 100 & 100 & 7 & 6 \\
\hline Erlangen-Nürnberg U & 500 & 500 & 43 & 50 \\
\hline Flensburg U & 400 & 600 & 24 & 40 \\
\hline Frankfurt/Main U & 1.100 & 500 & 42 & 21 \\
\hline Freiburg PH & 500 & 400 & 42 & 31 \\
\hline Freiburg U & 300 & 300 & 200 & 100 \\
\hline Gießen U & 600 & 700 & 67 & 47 \\
\hline Göttingen U & 1.000 & 300 & 167 & 50 \\
\hline Hagen U & 100 & 300 & 20 & 50 \\
\hline Halle-Wittenberg U & 2.800 & 3.200 & 140 & 229 \\
\hline Hamburg HSU* & 300 & - & 33 & - \\
\hline Hamburg U & 500 & 400 & 10 & 8 \\
\hline Hannover U & 700 & 200 & 47 & 13 \\
\hline Heidelberg $U^{* * *}$ & 200 & 0 & 100 & 0 \\
\hline Hildesheim U & 1.100 & 1.700 & 183 & 131 \\
\hline Jena U & 500 & 400 & 59 & 44 \\
\hline
\end{tabular}




\begin{tabular}{|c|c|c|c|c|}
\hline \multirow[t]{2}{*}{ Hochschule } & \multicolumn{2}{|c|}{$\begin{array}{l}\text { bewilligte Mittel } \\
\text { in Tausend Euro' }\end{array}$} & \multicolumn{2}{|c|}{$\begin{array}{l}\text { Bewilligungen pro Professur } \\
\text { in Tausend Euro }\end{array}$} \\
\hline & 2011-2013 & 2014-2016 & 2011-2013 & 2014-2016 \\
\hline Karlsruhe KIT* & 100 & - & 20 & - \\
\hline Kassel U & 900 & 500 & 72 & 31 \\
\hline Kiel U & 100 & 100 & 17 & 13 \\
\hline Koblenz-Landau U & 2.300 & 3.600 & 87 & 120 \\
\hline Köln U & 200 & 300 & 4 & 7 \\
\hline Konstanz U & 100 & 100 & 40 & 25 \\
\hline Leipzig** & - & 100 & - & 5 \\
\hline Lüneburg U* & 100 & - & 13 & - \\
\hline Magdeburg U* & 100 & - & 10 & - \\
\hline Mainz U & 1.200 & 1.400 & 96 & 88 \\
\hline Marburg U & 300 & 200 & 23 & 15 \\
\hline München LMU & 900 & 700 & 69 & 58 \\
\hline München TU & 1.400 & 1.000 & 80 & 91 \\
\hline Münster U & 1.400 & 800 & 85 & 40 \\
\hline Oldenburg U & 300 & 200 & 15 & 11 \\
\hline Osnabrück U & 300 & 700 & 29 & 58 \\
\hline Paderborn $\mathrm{U}^{* * *}$ & 300 & 0 & 29 & 0 \\
\hline Potsdam U & 400 & 700 & 29 & 33 \\
\hline Regensburg $U^{* * * *}$ & 0 & 200 & 0 & 25 \\
\hline Saarbrücken U & 200 & 600 & 40 & 120 \\
\hline Siegen $U$ & 200 & 200 & 10 & 9 \\
\hline Stuttgart U & 400 & 300 & 160 & 100 \\
\hline Trier $\mathrm{U}^{*}$ & 100 & - & 13 & - \\
\hline Tübingen U & 1.800 & 1.900 & 240 & 95 \\
\hline Ulm U* & 100 & - & 100 & - \\
\hline Wuppertal U & 300 & 500 & 21 & 28 \\
\hline Würzburg U & 900 & 100 & 75 & 8 \\
\hline
\end{tabular}

1 Zahlen gerundet

* Angaben für 2014-2016 fehlen

** Angaben für 2011-2013 fehlen

*** Aufgrund des Rundungsverfahrens auf 100.000 Euro können für 2014-2016 keine Mittel ausgewiesen werden.

${ }_{* * * *}$ Aufgrund des Rundungsverfahrens auf 100.000 Euro können für 2011-2013 keine Mittel ausgewiesen werden.

Quelle: DFG-Förderatlas 2015 und 2018; unveröffentlichtes Material des Statistischen Bundesamts; eigene Berechnungen 
Auch mit Blick auf die Standorte ist - wie schon bei Tabelle 5.3 erwähnt - ein Rückgang des bewilligten DFG-Volumens in der Erziehungswissenschaft zu beobachten. Bei mehr als der Hälfte der wissenschaftlichen Hochschulen ist jeweils eine Abnahme der DFG-Mittel zu verzeichnen.

Bei Betrachtung der DFG-stärksten Standorte sind auch dieses Mal die ähnlichen wissenschaftlichen Hochschulen wie im letzten Zeitraum vertreten. Nur in der Reihenfolge hat sich eine Verschiebung ergeben. So ist nun Koblenz-Landau an der Spitze, gefolgt von Halle-Wittenberg, Bamberg sowie Duisburg-Essen und Tübingen. In Relation zur Zahl der an der jeweiligen Hochschule vorhandenen erziehungswissenschaftlichen Professuren waren insbesondere die Universitäten Halle-Wittenberg, Bamberg, Hildesheim, Koblenz-Landau und Saarbrücken von 2013-2016 mit durchschnittlich 120.000 bis 229.000 Euro pro Professur besonders erfolgreich. Der etwas reduzierte Stellenwert des Standortes Bamberg im letzten Zeitraum jeweils Spitzenreiter - könnte auf die Gründung des LIfBi (Leibniz-Institut für Bildungsverläufe e.V.) im Jahre 2014 zurückzuführen sein. An diesem An-Institut der Universität Bamberg ist nun das Nationale Bildungspanel angesiedelt, das vorher über DFG-Mittel gefördert wurde.

\subsubsection{Forschungsverbünde}

Ein weiterer möglicher Indikator für die Forschungsstärke eines Fachs stellen Forschungsverbünde dar. Im Folgenden wird daher erstmals auf die Forschungsgruppen, Sonderforschungsbereiche und Graduiertenkollegs der DFG näher eingegangen.

Unter Forschungsgruppen werden enge Kooperationen von mehreren herausragenden Wissenschaftlerinnen und Wissenschaftlern zu einer besonderen Forschungsaufgabe verstanden. Ziel dieses mittelfristigen, meist auf acht Jahre angelegten (bis 1. Oktober 2018: bis zu sechs Jahre) Bündnisses ist es, Ergebnisse zu erhalten, die über die Möglichkeiten einer Einzelförderung hinausreichen. Eine Forschungsgruppe ist aus verschiedenen Modulen aufgebaut, die nach fachlichen Aspekten ausgewählt werden. Die DFG fördert zum 17. September 2019206 Forschungsgruppen, davon 47 im Bereich der Geistes- und Sozialwissenschaften. ${ }^{4}$ Eine genauere fachliche Zuordnung dieser Forschungsgruppen ist aber kaum möglich, da Angaben hierzu lediglich auf Ebene der in den Gruppen realisierten Teilprojekte vorliegen.

Sonderforschungsbereiche (SFB) sind Einrichtungen der Hochschulen, in denen Wissenschaftlerinnen und Wissenschaftler in einem fächerübergreifenden Forschungsprogramm zusammenarbeiten. Sie werden von der DFG bis zu zwölf Jahre gefördert. Hierbei können zwei Programmvarianten unterschieden werden. Zum einen der klassische Sonderforschungsbereich, der an

4 https://www.dfg.de/gefoerderte_projekte/programme_und_projekte/listen/index. jsp?id=FOR [Zugriff: 23.9.2019] 
einer Hochschule angesiedelt ist, und zum anderen der Sonderforschungsbereich (SFB/Transregio), in dem bis zu drei Hochschulen als Kooperationspartner mitwirken. Zum 17. September 2019 werden im Gesamten 278 Sonderforschungsbereiche von der DFG gefördert, die sich weiter in 198 klassische Sonderforschungsbereiche und $80 \mathrm{SFB} /$ Transregio unterteilen lassen. Dem Bereich Geistes- und Sozialwissenschaften können 36 Sonderforschungsbereiche zugeordnet werden, darunter $6 \mathrm{SFB}$ /Transregio. ${ }^{5}$ In der Erziehungswissenschaft wird derzeit kein Sonderforschungsbereich gefördert, während in den Vergleichsdisziplinen Psychologie 3, Sozialwissenschaft (inkl. Politikwissenschaft, Publizistik und Kommunikationswissenschaft) 6 und in den Wirtschaftswissenschaften 4 SFBs bzw. SFB/Transregios zu verzeichnen sind.

Zur Förderung des wissenschaftlichen Nachwuchses angelegte Einrichtungen der Hochschulen mit einer Laufzeit bis maximal neun Jahre werden als Graduiertenkollegs beschrieben. Im Rahmen eines thematischen Forschungsprogramms und strukturierten Förderkonzepts wird dabei die Qualifizierung der Promovierenden fokussiert. Insgesamt stehen 232 Graduiertenkollegs unter der Förderung der DFG. In den Geistes- und Sozialwissenschaften sind es 57 Kollegs, davon 5 internationale. ${ }^{6}$ Zum Stichtag (17.9.2019) wird in der Erziehungswissenschaft ein Graduiertenkolleg gefördert: »Doing Transitions. Formen der Gestaltung von Übergängen im Lebenslauf.« (Nr.: 2105, Ort: Frankfurt/Tübingen, Beginn: 2017). Abgeschlossene oder bereits bewilligte, aber noch nicht gestartete Kollegs sind hier nicht aufgelistet. In der Psychologie sind 3, in den Sozialwissenschaften 8 und in den Wirtschaftswissenschaften 7 Graduiertenkollegs gelistet.

Insgesamt lässt sich festhalten, dass das Drittmittelvolumen in der Erziehungswissenschaft sowohl nominal als auch relational kontinuierlich angewachsen ist, die über die DFG eingeforderten Mittel sich aber genau entgegengesetzt dieses Trends entwickeln. Die fehlende Beteiligung der Erziehungswissenschaft an großen Forschungsverbünden mag ein Grund hierfür sein, dürfte insbesondere die aktuelle negative Entwicklung aber kaum erklären können. Hierzu wären genauere Analysen zu Antragszahlen und Ablehnungsquoten in der Erziehungswissenschaft im Vergleich zu anderen Disziplinen wünschenswert, auch um mehr Transparenz in der Förderpolitik der DFG herzustellen.

\subsection{Publikationskulturen in der Erziehungswissenschaft}

Fachveröffentlichungen sind der wohl bedeutendste Weg, Forschungsergebnisse und wissenschaftliche Diskussionsbeiträge zu kommunizieren und auch zu archivieren. Die Wege und Formate, welche hierfür genutzt werden, sind daher

5 https://www.dfg.de/gefoerderte_projekte/programme_und_projekte/listen/index. jsp?id=SFB [Zugriff: 23.9.2019]

6 https://www.dfg.de/gefoerderte_projekte/programme_und_projekte/listen/index. jsp?id=GRK [Zugriff: 23.9.2019] 
bedeutsame Indikatoren für die Art, wie innerhalb einer Disziplin und über ihre Grenzen hinaus wissenschaftliche Kommunikation stattfindet. Gleichzeitig spiegeln sich in den Veröffentlichungsformen und -organen auch von außen an die Disziplin gerichtete Anforderungen und Erwartungen wider, wenn z.B. vor dem Hintergrund eines New Public Management und veränderten Governance-Regimen im Wissenschaftsbereich (vgl. Wilkesmann 2016) unterschiedliche Publikationsformen verschieden bewertet werden und diese Bewertungen immer stärker auch zu Kriterien für die Bewertung wissenschaftlicher Leistung und der Zuweisung von Ressourcen werden. Zunehmend scheinen vor dem Hintergrund einer Vermessung wissenschaftlicher Leistungen die Frage danach, wo (oder auch mit wem) publiziert wird, und die Frage nach der Häufigkeit von Zitationen die Frage nach der darüber hinausgehenden inhaltlichen Qualität der jeweiligen Veröffentlichung zu verdrängen. Auch wenn die Belastbarkeit solcher Indikatoren immer wieder zum Gegenstand kritischer Auseinandersetzungen wird, scheint der Bedarf an quantifizierbaren Indikatoren, wie Publikationen oder Drittmittel (s.o.), so groß zu sein, dass lediglich deren weitere Ausdifferenzierung, aber nicht mehr die Frage nach der grundsätzlichen Möglichkeit wissenschaftliche Produktivität zu vermessen, auf der wissenschaftspolitischen Agenda steht (vgl. z.B. Wissenschaftsrat 2013). Vor diesem Hintergrund stehen insbesondere junge Wissenschaftlerinnen und Wissenschaftler immer mehr unter Druck, in den jeweiligen (subdisziplinären) wissenschaftlichen Kulturen geltenden Kriterien entsprechend sichtbar zu publizieren. Daher scheint es aufschlussreich, das Publikationsverhalten von Nachwuchswissenschaftlerinnen und Nachwuchswissenschaftlern mit dem von professoralen Mitgliedern der Scientific Community zu vergleichen.

\subsubsection{Datenbasis}

Seit der ersten Ausgabe von 2000 nutzt der Datenreport die »FIS Bildung Literaturdatenbank « zur Analyse von Publikationskulturen in der Erziehungswissenschaft (vgl. Weishaupt/Merkens 2000). Die entsprechenden Daten werden vom DIPF|Leibniz-Institut für Bildungsforschung und Bildungsinformation bereitgestellt. ${ }^{7}$ In diesem Zeitraum haben sich nicht nur Publikationsformen und -strategien verändert, sondern auch die Systematik, mit der Veröffentlichungen in der Datenbank erfasst werden, wurde kontinuierlich weiterentwickelt. Allerdings ist die Anzahl der erfassten Publikationen seit Jahren rückläufig, was wohl eher als ein Problem der Erfassungssystematik zu deuten und allenfalls in Teilen auf einen tatsächlichen Rückgang der Publikationsaktivitäten im Feld zurückzuführen ist. Besonders betroffen hiervon dürften die englisch- und anderssprachigen Publika-

7 Wir danken insbesondere Frau Katrin Wicker vom DIPF|Leibniz-Institut für Bildungsforschung und Bildungsinformation für die unkomplizierte Bereitstellung der Daten und die wertvollen Hinweise zur Erhebungssystematik. 
tionen deutscher Autorinnen und Autoren sein. Hier ist die Zahl erfasster Beiträge besonders deutlich rückläufig und eine nur annähernd vollständige Dokumentation in einer Datenbank scheint angesichts der Vielfalt und Unübersichtlichkeit der internationalen Publikationslandschaft auch kaum zu realisieren. Entsprechend sind die Daten zu englischsprachigen Publikationen mit besonderer Vorsicht zu interpretieren. Im Hinblick auf die deutschsprachigen Veröffentlichungen hingegen gibt es zwar Hinweise auf eine Untererfassung, aber zumindest keine aus den Daten erkennbaren Hinweise auf systematische Verzerrungen.

Dabei ist zu berücksichtigen, dass kontinuierlich auch ältere Veröffentlichungen nachgetragen werden und daher der Korpus auch älterer Jahrgänge weiter aufwächst. Insofern scheint es zunächst nachvollziehbar, dass gerade die aktuellsten Jahrgänge noch etwas weniger Veröffentlichungen umfassen als die Älteren. Der sich in Tabelle 5.5 abzeichnende kontinuierliche Abfall in »FIS Bildung « erfasster Publikationen lässt sich damit aber kaum erklären. Trotz einer offensichtlich weniger stringenten Erfassung von Publikationen der »FIS Bildung Literaturdatenbank «, scheint auch ein tatsächlich reduziertes Publikationsaufkommen in der deutschen Erziehungswissenschaft nicht völlig unplausibel. Angesichts wissenschaftspolitischer Steuerungsmaßnahmen (z.B. seitens der DFG), die eine stärkere Konzentration auf wissenschaftlich besonders hochwertige und international sichtbare Publikationen statt rein auf Quantität ausgerichtete Publikationsstrategien forcieren, könnte man hier einen Steuerungseffekt vermuten. Zwischen 2010 und 2018 ist die Anzahl der in »FIS Bildung « erfassten Publikationen um fast $60 \%$ gesunken, wobei der Rückgang bei den Zeitschriftenpublikationen ( $-36 \%$ ) geringer ausfällt als im Bereich der Buchpublikationen ( $-72 \%)$ und den Beiträgen in Sammelbänden (-76 \%).

Tabelle 5.5: Anzahl der in »FIS Bildung« erfassten Publikationen 2007-2018

\begin{tabular}{|c|c|c|c|c|c|c|c|c|c|c|c|c|}
\hline & 2007 & 2008 & 2009 & 2010 & 2011 & 2012 & 2013 & 2014 & 2015 & 2016 & 2017 & 2018 \\
\hline $\begin{array}{l}\text { Monografien, } \\
\text { Herausgeber- } \\
\text { bände }\end{array}$ & 10.655 & 10.454 & 10.296 & 10.577 & 9.795 & 8.919 & 7.042 & 5.161 & 4.753 & 4.572 & 4.080 & 2.986 \\
\hline Buchbeiträge & 4.606 & 5.185 & 3.996 & 5.534 & 4.342 & 4.182 & 3.833 & 4.292 & 3.175 & 2.936 & 2.353 & 1.349 \\
\hline $\begin{array}{l}\text { Zeitschriften- } \\
\text { artikel }\end{array}$ & 13.848 & 14.240 & 11.977 & 12.102 & 11.537 & 10.831 & 10.982 & 10.787 & 9.903 & 9.617 & 9.089 & 7.758 \\
\hline gesamt & 29.109 & 29.879 & 26.269 & 28.213 & 25.674 & 23.932 & 21.857 & 20.240 & 17.831 & 17.125 & 15.522 & 12.093 \\
\hline
\end{tabular}

Quelle: Daten aus »FIS Bildung«, eigene Berechnungen

Sowohl vor dem Hintergrund hochschulpolitischer Vorgaben als auch der Ausgestaltung der Hochschullandschaft sind Unterschiede in den Publikationskulturen zwischen verschiedenen Bundesländern zu erwarten. Um Aussagen hierüber, aber auch zu Differenzen zwischen professoralen und nicht-professoralen Mitgliedern der Scientific Community treffen zu können, wurden die Daten aus der »FIS Bildung Literaturdatenbank« mit dem Mitgliederverzeichnis der DGfE gematcht. Dadurch ist es möglich, einzelne Autorinnen und Autoren anhand ih- 
res Titels zu differenzieren sowie jeweiligen Regionen zuzuordnen. Die im Datenreport 2008 erstmals vorgenommene Betrachtung länderbezogener Publikationskulturen lässt sich so replizieren (siehe Tabelle 5.6a), wobei hierfür - analog zum Datenreport 2008 - u.a. ein Drei-Jahres-Zeitraum ausgewählt wurde. Entsprechend der Zielsetzung dieses Datenreports, auch langfristige Entwicklungen in den Blick zu nehmen, werden für die Analyse auf Länderebene zunächst zwei Zeiträume im Abstand von 13 Jahren herangezogen. Dies begründet sich aus älteren für den Datenreport vorgenommenen Publikationsanalysen für die Jahre 2003-2005, welchen die aktuellsten Daten gegenübergestellt werden.

\subsubsection{Publikationen im Ländervergleich}

Die Ergebnisse spiegeln einerseits länderübergreifende Trends (geringere Anzahl an Publikationen insgesamt) wider und verweisen andererseits auf länderspezifische Entwicklungen, deren Ursachen hier nicht aufzuklären sind. Es ist aber davon auszugehen, dass die quantitative Stärke erziehungswissenschaftlicher Standorte in den jeweiligen Ländern sowie subdisziplinäre Schwerpunktbildungen an den Standorten und deren Veränderung hier eine wesentliche Rolle spielen. Besonders deutlich fällt der Rückgang von erfassten Publikationen der DGfE-Mitglieder in Bremen (-64 \%), Thüringen (-53 \%) und Bayern (-45\%) sowie Sachsen-Anhalt (-42\%), Thüringen (-41\%) und Mecklenburg-Vorpommern (-40\%) aus. Während die Zahl von Veröffentlichungen in Hessen stabil blieb, hat sie sich lediglich in Baden-Württemberg im Vergleichszeitraum positiv entwickelt $(+7 \%)$.

Insbesondere der Anteil von Zeitschriftenbeiträgen hat sich regional sehr unterschiedlich entwickelt. Während der Anteil von Veröffentlichungen in Zeitschriften in Bremen (+14 \%), Sachsen und Thüringen (je +9\%) erkennbar zugenommen hat, ist er in anderen Bundesländern wie Schleswig-Holstein $(-24 \%)$, Brandenburg (-18 \%), Hamburg (-13\%), Sachsen-Anhalt (-12\%) sowie Sachsen und Rheinland-Pfalz/Saarland (-11 \%) deutlich zurückgegangen. Eine Rolle spielt hierbei vermutlich das Profil der im jeweiligen Bundesland beteiligten Hochschulen und erziehungswissenschaftlichen Fachbereiche, aber auch deren Rekrutierungsstrategien und internen Steuerungsmechanismen könnten einen Erklärungsbeitrag für die Unterschiede und Veränderungen liefern.

Betrachtet man die Publikationen der DGfE-Mitglieder insgesamt, so ist hier ebenfalls ein entsprechender Rückgang erkennbar (siehe Tabelle 5.6a). In den meisten Bundesländern ist die Anzahl von Publikationen in den Vergleichszeiträumen (2003-2005 und 2016-2018) gesunken. Betrachtet man hingegen die Veröffentlichungen pro aktiv publizierendem DGfE-Mitglied ${ }^{8}$,

8 Ausgeschlossen wurden Mitglieder, die in dem betrachteten Drei-Jahres-Zeitraum an keiner einzigen Publikation beteiligt waren, um Verzerrungen durch Fördermitglieder und Mitglieder, die nicht (mehr) aktiv in der Wissenschaft tätig sind, zu vermeiden. 
lässt sich in nahezu allen Ländern ein Zuwachs konstatieren, d.h. die erfassten Publikationen wurden von weniger Autorinnen und Autoren verfasst, die dann aber an mehr Veröffentlichungen beteiligt waren. Einzige Ausnahme hier ist Hamburg, wo die Anzahl von Publikationen pro aktivem DGfE-Mitglied 2003-2005 weit über dem Durchschnitt lag und trotz eines leichten Rückgangs 13 Jahre später immer noch überdurchschnittlich ist. Besonders hohe Zuwächse hinsichtlich der Anzahl von Publikationen pro DGfE-Mitglied sind für Sachsen-Anhalt $(0,89)$, Baden-Württemberg $(0,80)$ und Schleswig-Holstein $(0,73)$ sowie Sachsen $(0,69)$ und Hessen $(0,67)$ zu verzeichnen.

Tabelle 5.6a: Publikationstätigkeiten in der Erziehungswissenschaft nach Bundesländern 2003-2005 und 2016-2018

\begin{tabular}{|c|c|c|c|c|c|c|c|c|}
\hline \multirow[b]{2}{*}{ Bundesland } & \multicolumn{2}{|c|}{$\begin{array}{l}\text { erfasste } \\
\text { Publikationen } \\
\text { insgesamt }\end{array}$} & \multicolumn{2}{|c|}{$\begin{array}{l}\text { Anteil der } \\
\text { Zeitschriftenver- } \\
\text { öffentlichungen }\end{array}$} & \multicolumn{2}{|c|}{$\begin{array}{l}\text { Publikationen je } \\
\text { hauptberuflicher } \\
\text { Wissenschaftler }\end{array}$} & \multicolumn{2}{|c|}{$\begin{array}{l}\text { Zeitschriften- } \\
\text { beiträge } \\
\text { je Professur }\end{array}$} \\
\hline & $\begin{array}{l}2003- \\
2005\end{array}$ & $\begin{array}{l}2016- \\
2018\end{array}$ & $\begin{array}{l}2003- \\
2005\end{array}$ & $\begin{array}{l}2016- \\
2018\end{array}$ & $\begin{array}{l}2003- \\
2005\end{array}$ & $\begin{array}{l}2016- \\
2018\end{array}$ & $\begin{array}{l}2003- \\
2005\end{array}$ & $\begin{array}{l}2016- \\
2018\end{array}$ \\
\hline $\begin{array}{l}\text { Baden- } \\
\text { Württemberg }\end{array}$ & 421 & 449 & $63 \%$ & $59 \%$ & 1,38 & 2,18 & 1,17 & 1,35 \\
\hline Bayern & 616 & 337 & $60 \%$ & $60 \%$ & 1,53 & 1,81 & 1,58 & 1,29 \\
\hline Berlin & 488 & 356 & $47 \%$ & $49 \%$ & 1,92 & 2,37 & 1,42 & 1,50 \\
\hline Brandenburg & 88 & 52 & $62 \%$ & $44 \%$ & 1,44 & 1,93 & 1,30 & 1,06 \\
\hline Bremen & 154 & 55 & $51 \%$ & $65 \%$ & 1,60 & 1,67 & 1,65 & 1,24 \\
\hline Hamburg & 335 & 228 & $64 \%$ & $51 \%$ & 2,64 & 2,28 & 1,67 & 1,36 \\
\hline Hessen & 479 & 479 & $59 \%$ & $52 \%$ & 1,84 & 2,51 & 1,80 & 1,54 \\
\hline $\begin{array}{l}\text { Mecklenburg- } \\
\text { Vorpommern }\end{array}$ & 35 & 21 & $52 \%$ & $50 \%$ & 0,91 & 1,24 & 1,50 & 0,97 \\
\hline Niedersachsen & 484 & 342 & $64 \%$ & $60 \%$ & 1,59 & 1,87 & 1,26 & 1,54 \\
\hline $\begin{array}{l}\text { Nordrhein- } \\
\text { Westfalen }\end{array}$ & 1.139 & 1.079 & $59 \%$ & $53 \%$ & 1,69 & 2,24 & 1,36 & 1,41 \\
\hline $\begin{array}{l}\text { Rheinland-Pfalz/ } \\
\text { Saarland' }\end{array}$ & 278 & 193 & $60 \%$ & $49 \%$ & 1,50 & 2,03 & 1,72 & 1,29 \\
\hline Sachsen & 135 & 96 & $54 \%$ & $63 \%$ & 0,86 & 1,55 & 1,22 & 1,27 \\
\hline Sachsen-Anhalt & 158 & 91 & $56 \%$ & $42 \%$ & 1,25 & 2,34 & 0,84 & 1,09 \\
\hline Schleswig-Holstein & 43 & 54 & $71 \%$ & $47 \%$ & 0,68 & 1,41 & 0,50 & 0,79 \\
\hline Thüringen & 128 & 60 & $52 \%$ & $61 \%$ & 1,57 & 1,81 & 1,33 & 1,18 \\
\hline
\end{tabular}

1 Wegen sonst zu kleiner Fallzahlen wurden die Länder Rheinland-Pfalz und Saarland hier zusammengenommen.

Quelle: Daten aus »FIS Bildung«, DGfE-Mitgliederliste; eigene Berechnungen

Unterschiedlich entwickelt hat sich die Anzahl von Zeitschriftenpublikationen pro aktiv publizierender Professorin bzw. publizierendem Professor. In Schleswig-Holstein $(+0,29)$, Niedersachsen $(+0,28)$, Sachsen-Anhalt $(+0,25)$ und Baden-Württemberg $(+0,18)$ sowie in Berlin $(+0,08)$, Sachsen $(+0,05)$ und 
Nordrhein-Westfalen $(+0,05)$ ist die Anzahl von Zeitschriftenpublikationen professoraler DGfE-Mitglieder zwischen den beiden Betrachtungszeiträumen gestiegen. Zurückgegangen ist dieser Indikator dagegen in MecklenburgVorpommern $(-0,53)$, Rheinland-Pfalz/Saarland $(-0,43)$, Bremen $(-0,31)$, Bayern $(-0,29)$, Hamburg $(-0,29)$, Hessen $(-0,26)$, Brandenburg $(-0,24)$ und Thüringen $(-0,15)$. Dabei sind diese Daten wiederum mit großer Vorsicht zu interpretieren, da nicht nur die kontinuierliche Abnahme in der »FIS Bildung Literaturdatenbank« erfasster Publikationen hier einen Effekt haben dürfte, sondern auch eine geringfügige Veränderung der Anzahl von Professorinnen und Professoren in einem Bundesland hier schon starke Effekte haben kann. Darüber hinaus umfasst die Mitgliederliste der DGfE auch Emeriti bzw. Professorinnen und Professoren im Ruhestand, die sich anhand der Daten aber nicht als solche identifizieren lassen.

Die aus den ersten beiden Datenreporten 2000 (Weishaupt/Merkens 2000) und 2004 (Kraul/Schulzeck/Weishaupt 2004) vorliegenden Publikationsanalysen lassen sich aufgrund eines anderen Zählverfahrens (hier wurden Publikationen mit mehreren Autorinnen und Autoren mehrfach gezählt) nur noch eingeschränkt replizieren. Für zwei bundeslandbezogene Indikatoren konnte das allerdings auch auf aktueller Basis umgesetzt werden. Für den Vergleich mit den in frühen Datenreporten publizierten Indikatoren wurde nun ein FünfJahres-Zeitraum in den Blick genommen (2014-2018).

Hier bestätigt sich einerseits der beschriebene quantitative Rückgang von in der "FIS Bildung Literaturdatenbank» erfassten Publikationen auch für Professorinnen und Professoren, zum anderen werden Verschiebungen zwischen den Ländern sichtbar (siehe Tabelle 5.6b). Während einige Länder mit einer in der Vergangenheit besonders publikationsaktiven Professorenschaft aktuell nur noch im Mittelfeld liegen (z.B. Bremen oder RheinlandPfalz/Saarland) konnte Sachsen-Anhalt seine Spitzenposition behaupten und auch Berlin sowie Nordrhein-Westfalen gehören schon traditionell zu den Ländern mit höherem gemeldetem Publikationsaufkommen in der Professorenschaft. Interessant ist auch der Anteil von Zeitschriftenpublikationen der DGfE-Mitglieder, der im Bundesdurchschnitt über den betrachteten Zwanzig-Jahres-Zeitraum sehr stabil geblieben ist, auf Länderebene aber erheblich schwankt. Der Anteil von Zeitschriftenpublikationen hat in Baden-Württemberg, Berlin, Bremen und Sachsen deutlich zugenommen und hat sich im gleichen Zeitraum in Brandenburg, Hamburg, Mecklenburg-Vorpommern und Sachsen-Anhalt gegenläufig entwickelt, wobei sich viele Länder auch vor allem durch Diskontinuität dieser Entwicklungsverläufe auszeichnen. Offensichtlich scheint es sich auf Landesebene um einen recht instabilen Indikator $\mathrm{zu}$ handeln, der u.a. auf die Berufungspolitik an einzelnen Standorten zurückzuführen sein kann. 
Tabelle 5.6b: Publikationstätigkeiten in der Erziehungswissenschaft nach Bundesländern 1993-1998, 1998-2002 und 2014-2018

\begin{tabular}{|c|c|c|c|c|c|c|}
\hline \multirow[b]{2}{*}{ Bundesland } & \multicolumn{3}{|c|}{$\begin{array}{l}\text { gemeldete } \\
\text { Veröffentlichungen } \\
\text { je Professur }\end{array}$} & \multicolumn{3}{|c|}{$\begin{array}{l}\text { Anteil } \\
\text { Zeitschriften- } \\
\text { veröffentlichungen }\end{array}$} \\
\hline & $\begin{array}{l}1993- \\
1998\end{array}$ & $\begin{array}{l}1998- \\
2002\end{array}$ & $\begin{array}{l}2014- \\
2018\end{array}$ & $\begin{array}{l}1993- \\
1998\end{array}$ & $\begin{array}{l}1998- \\
2002\end{array}$ & $\begin{array}{l}2014- \\
2018\end{array}$ \\
\hline Baden-Württemberg & 7,1 & 5,7 & 3,9 & $50 \%$ & $59 \%$ & $58 \%$ \\
\hline Bayern & 9,1 & 9,4 & 3,6 & $55 \%$ & $57 \%$ & $55 \%$ \\
\hline Berlin & 9,3 & 9,3 & 5,1 & $44 \%$ & $47 \%$ & $48 \%$ \\
\hline Brandenburg & 7,9 & 7,1 & 4,7 & $62 \%$ & $50 \%$ & $40 \%$ \\
\hline Bremen & 7,2 & 9,9 & 3,1 & $40 \%$ & $46 \%$ & $62 \%$ \\
\hline Hamburg & 9,3 & 9,4 & 4,6 & $57 \%$ & $48 \%$ & $46 \%$ \\
\hline Hessen & 9,4 & 8,4 & 4,5 & $53 \%$ & $59 \%$ & $53 \%$ \\
\hline Mecklenburg-Vorpommern & 8,0 & 5,8 & 2,3 & $64 \%$ & $40 \%$ & $44 \%$ \\
\hline Niedersachsen & 9,5 & 7,6 & 4,5 & $54 \%$ & $62 \%$ & $54 \%$ \\
\hline Nordrhein-Westfalen & 10,0 & 9,0 & 4,7 & $52 \%$ & $49 \%$ & $51 \%$ \\
\hline Rheinland-Pfalz/Saarland' & 12,0 & 9,5 & 4,2 & $55 \%$ & $52 \%$ & $48 \%$ \\
\hline Sachsen & 7,6 & 8,6 & 2,8 & $51 \%$ & $51 \%$ & $58 \%$ \\
\hline Sachsen-Anhalt & 12,0 & 9,0 & 6,1 & $47 \%$ & $56 \%$ & $39 \%$ \\
\hline Schleswig-Holstein & 7,1 & 5,9 & 2,9 & $52 \%$ & $52 \%$ & $49 \%$ \\
\hline Thüringen & 9,7 & 9,7 & 4,0 & $51 \%$ & $36 \%$ & $54 \%$ \\
\hline BRD & 9,1 & 8,4 & 4,3 & $52 \%$ & $53 \%$ & $52 \%$ \\
\hline
\end{tabular}

1 Wegen sonst zu kleiner Fallzahlen wurden die Länder Rheinland-Pfalz und Saarland hier zusammengenommen.

Quelle: Daten aus »FIS Bildung«, DGfE-Mitgliederliste; eigene Berechnungen

Im Datenreport 2016 wurde den Publikationsaktivitäten erziehungswissenschaftlicher Professorinnen und Professoren besondere Aufmerksamkeit gewidmet. Dieser Fokus ist v.a. insofern berechtigt, als die in der »FIS Bildung Literaturdatenbank « erfassten Publikationen keineswegs nur von im Wissenschaftssystem tätigen Personen verfasst sind, sondern vielfach auch von Expertinnen und Experten aus der Praxis. Gleichzeitig verändern sich Publikationsaktivitäten im Karriereverlauf und interindividuelle Unterschiede hinsichtlich der wissenschaftlichen Produktivität nehmen zu (vgl. Röbken 2014). Vor diesem Hintergrund werden hier auch einige Analysen, die im Datenreport 2016 erstmalig für die Gruppe der Professorinnen und Professoren vorgenommen wurden, weitergeführt. Allerdings bestand aufgrund eines Drittmittelprojekts (vgl. Tafertshofer/Werner/Schmidt-Hertha 2018) für die Publikationsdaten 2010 bis 2013 die Möglichkeit, diese nicht nur 
mit der DGfE-Mitgliederliste abzugleichen, sondern auch mit dem Hochschullehrerverzeichnis und zusätzlichen Internetrecherchen, was für die aktuelleren Jahrgänge so nicht mehr möglich war. Da für die Jahre 2014 bis 2017 nur die Mitgliederliste der DGfE als Quelle erziehungswissenschaftlicher Professuren zur Verfügung stand, ist von einer Untererfassung auszugehen. Insbesondere in einigen Überschneidungsbereichen zu anderen Fächern (z.B. der empirischen Bildungsforschung) ist davon auszugehen, dass nicht alle in erziehungswissenschaftlichen Instituten verankerten Professorinnen und Professoren auch DGfE-Mitglieder sind. Diese möglichen Verzerrungen sind bei der Dateninterpretation zu beachten.

\subsubsection{Publikationen von Professorinnen und Professoren der Erziehungswissenschaft}

Entsprechend den in der »FIS Bildung Literaturdatenbank« erfassten Publikationen insgesamt, sind auch die Publikationen der Professorinnen und Professoren rückläufig. Im Vergleich von Vier-Jahres-Zeiträumen sind zwischen dem Berichtszeitraum des Datenreports 2012 (Schmidt-Hertha 2012) und dem aktuellsten Zeitraum acht Jahre später die Publikationen pro Professur um mehr als ein Viertel (27,4 \%) zurückgegangen. Gleichzeitig hat sich der Anteil von Zeitschriftenbeiträgen sukzessive erhöht und macht inzwischen mehr als die Hälfte aller Publikationen von Professorinnen und Professoren der Erziehungswissenschaft aus (siehe Tabelle 5.7).

Tabelle 5.7: Gemeldete Veröffentlichungen hauptberuflicher Professorinnen und Professoren 2006-2009, 2010-2013 und 2014-2017 nach Publikationsform

\begin{tabular}{|c|c|c|c|c|}
\hline & & 2006-2009 & 2010-2013 & 2014-2017 \\
\hline \multirow{3}{*}{ Monografien } & Mittelwert & 0,69 & 0,62 & 0,40 \\
\hline & Standardabw. & 1,11 & 1,03 & 0,73 \\
\hline & Anteil an Publikationen & $12,9 \%$ & $12,2 \%$ & $10,3 \%$ \\
\hline \multirow{3}{*}{ Buchbeiträge } & Mittelwert & 2,33 & 2,13 & 1,55 \\
\hline & Standardabw. & 4,00 & 3,20 & 2,42 \\
\hline & Anteil an Publikationen & $43,5 \%$ & $41,8 \%$ & $39,8 \%$ \\
\hline \multirow{3}{*}{ Zeitschriftenartikel } & Mittelwert & 2,33 & 2,34 & 1,95 \\
\hline & Standardabw. & 3,18 & 2,78 & 2,29 \\
\hline & Anteil an Publikationen & $43,5 \%$ & $46,0 \%$ & $50,1 \%$ \\
\hline \multirow{2}{*}{ Publikationen insgesamt } & Mittelwert & 5,36 & 5,09 & 3,89 \\
\hline & Standardabw. & 6,57 & 5,52 & 4,19 \\
\hline
\end{tabular}

Quelle: Daten aus »FIS Bildung«; Bildungsserver, DGfE-Mitgliederliste; 2010-2013: Hochschullehrer Verzeichnis 2013, manuelle Internetrecherche; eigene Berechnungen 
Ein deutlich erkennbarer Trend der letzten Jahre ging in Richtung Mehrautorenschaften (Schmidt-Hertha/Tafertshofer/Tippelt 2016) und scheint sich nach einem Bruch in den Daten 2014 - mit Blick auf Professorinnen und Professoren in der Erziehungswissenschaft auch aktuell weiter fortzusetzen (siehe Tabelle 5.8). Der Anteil von in Alleinautorenschaft verfassten Beiträgen sank innerhalb von acht Jahren von $47,7 \%$ auf $26,8 \%$ und hat sich damit nahezu halbiert. Veröffentlichungen mit zwei Autorinnen und/oder Autoren sind parallel dazu zur dominanten Publikationsform professoraler DGfE-Mitglieder aufgestiegen und machten in den letzten Jahren stabil ca. $40 \%$ aller für diese Gruppe erfassten Veröffentlichungen aus. Deutlich gewachsen ist auch der Anteil von Veröffentlichungen mit drei Verfasserinnen und/oder Verfassern, wohingegen sich die insbesondere in naturwissenschaftlichen Disziplinen bekannte Kultur von Publikationen mit vielen Autorinnen und Autoren in der Erziehungswissenschaft so noch nicht durchzusetzen scheint.

Tabelle 5.8: Zahl der Autorinnen und Autoren bei Publikationen hauptberuflicher Professorinnen und Professoren 2010-2017 sowie prozentualer Anteil bezogen auf das jeweilige Jahr

\begin{tabular}{ccccccccc}
$\begin{array}{c}\text { Zahl der } \\
\text { Autoren }\end{array}$ & 2010 & 2011 & 2012 & 2013 & 2014 & 2015 & 2016 & 2017 \\
\hline 1 & 742 & 627 & 619 & 553 & 409 & 126 & 67 & 41 \\
& $47,7 \%$ & $46,0 \%$ & $46,0 \%$ & $41,2 \%$ & $52,5 \%$ & $37,1 \%$ & $26,5 \%$ & $26,8 \%$ \\
\hline 2 & 415 & 377 & 354 & 375 & 243 & 137 & 105 & 60 \\
& $26,7 \%$ & $27,7 \%$ & $26,3 \%$ & $27,9 \%$ & $31,2 \%$ & $40,3 \%$ & $41,5 \%$ & $39,2 \%$ \\
\hline 3 & 193 & 169 & 189 & 203 & 68 & 44 & 62 & 30 \\
& $12,4 \%$ & $12,4 \%$ & $14,0 \%$ & $15,1 \%$ & $8,7 \%$ & $12,9 \%$ & $24,5 \%$ & $19,6 \%$ \\
\hline 5 & 84 & 66 & 72 & 87 & 32 & 17 & 11 & 8 \\
mend & 122 & 124 & 112 & 124 & 27 & 16 & 8 & 14 \\
\hline & $7,8 \%$ & $9,1 \%$ & $8,3 \%$ & $9,2 \%$ & $3,5 \%$ & $4,7 \%$ & $3,2 \%$ & $9,2 \%$ \\
\hline
\end{tabular}

Quelle: Daten aus »FIS Bildung«; DGfE-Mitgliederliste; 2010-2013: Hochschullehrerverzeichnis 2013, manuelle Internetrecherche; eigene Berechnungen

Wie mit Blick auf die Zahl von Autorinnen und Autoren ist auch bei der Analyse der Publikationssprache zu beachten, dass nicht alle Professorinnen und Professoren, die bislang in der Erziehungswissenschaft verankert waren bzw. organisational auch weiter sind, Mitglied der DGfE sind. Veränderungen in der Zuordnung zu Fachgesellschaften könnten gerade im Hinblick auf Veröffentlichungen relevante Effekte haben, da z.B. der Bereich der empirischen Bildungsforschung, der mit der Gründung der GEBF 2012 eine alternative fachge- 
sellschaftliche Vertretung hat, traditionell eine stärkere Nähe zur Psychologie und deren Publikationskultur aufweist.

Unter anderem sind in der empirischen Bildungsforschung englischsprachige Publikationen besonders verbreitet, sodass die hohe Stabilität der Dominanz deutschsprachiger Veröffentlichungen in der erziehungswissenschaftlichen Professorenschaft auch mit diesen Veränderungen durch eine neue Fachgesellschaft in Zusammenhang stehen könnte. Festzuhalten ist angesichts der Daten aus »FIS Bildung " - wenngleich diese hinsichtlich der Erfassung außerhalb Deutschlands erschienener Publikationen nur eingeschränkt belastbar sind -, dass die dominante Wissenschaftssprache in der deutschen Erziehungswissenschaft weiterhin Deutsch ist und sich zumindest auf professoraler Ebene bislang keine Trendwende hin zu englischsprachigen Publikationen feststellen lässt.

Tabelle 5.9: Veröffentlichungen hauptberuflicher erziehungswissenschaftlicher Professorinnen und Professoren 2010-2017 nach Publikationssprache

\begin{tabular}{|c|c|c|c|c|c|c|c|c|}
\hline & 2010 & 2011 & 2012 & 2013 & 2014 & 2015 & 2016 & 2017 \\
\hline Deutsch & 1.489 & 1.301 & 1.281 & 1.271 & 751 & 329 & 226 & 144 \\
\hline $\begin{array}{l}\text { Anteil aller } \\
\text { Publikationen }\end{array}$ & $95,7 \%$ & $95,5 \%$ & $95,2 \%$ & $94,7 \%$ & $96.2 \%$ & $96,8 \%$ & $97,0 \%$ & $94,1 \%$ \\
\hline Englisch & 62 & 58 & 62 & 69 & 29 & 11 & 7 & 9 \\
\hline $\begin{array}{l}\text { Anteil aller } \\
\text { Publikationen }\end{array}$ & $4,0 \%$ & $4,3 \%$ & $4,6 \%$ & $5,1 \%$ & $3,7 \%$ & $3,2 \%$ & $3,0 \%$ & $5,9 \%$ \\
\hline Sonstige & 5 & 4 & 3 & 2 & 1 & - & - & - \\
\hline $\begin{array}{l}\text { Anteil aller } \\
\text { Publikationen }\end{array}$ & $0,3 \%$ & $0,3 \%$ & $0,2 \%$ & $0,1 \%$ & $0,1 \%$ & & & \\
\hline gesamt & 1.556 & 1.363 & 1.346 & 1.342 & 781 & 340 & 233 & 153 \\
\hline $\begin{array}{l}\text { Anteil aller } \\
\text { Publikationen }\end{array}$ & $100 \%$ & $100 \%$ & $100 \%$ & $100 \%$ & $100 \%$ & $100 \%$ & $100 \%$ & $100 \%$ \\
\hline
\end{tabular}

Quelle: »FIS Bildung«, DGfE-Mitgliederliste; 2010-2013: Hochschullehrerverzeichnis 2013, manuelle Internetrecherche; eigene Berechnungen

\subsection{Fazit}

Mit Blick auf die in den vergangenen zwanzig Jahren im Datenreport dokumentierten Forschungsindikatoren lassen sich sowohl deutliche Trends als auch diskontinuierliche Entwicklungen nachvollziehen. So spiegeln sich die Veränderungen in der Hochschulfinanzierung - insbesondere die zunehmende Forschungsfinanzierung über Drittmittel - auch in den Zahlen der Erzie- 
hungswissenschaft wider. Dabei ist ein kontinuierlich anwachsendes Volumen von Drittmitteln sowohl pro Professur als auch insgesamt ebenso festzustellen wie eine - im Vergleich zu anderen Fächern - größere Breite von Drittmittelquellen. Auffallend ist hier insbesondere der im Fächervergleich geringere Anteil der Erziehungswissenschaft an DFG-Mitteln, wobei die Frage offen bleibt, ob weniger DFG-Mittel beantragt werden, weil hinreichend alternative Quellen zur Forschungsfinanzierung (insbesondere durch das BMBF) zur Verfügung stehen, oder ob andere Quellen genutzt werden, weil Mittel der DFG für Erziehungswissenschaftlerinnen und Erziehungswissenschaftler besonders schwer zu akquirieren sind.

Weniger klar sind die Entwicklungen in den Publikationskulturen, was aber zu erheblichem Teil mit an den verfügbaren Literaturdatenbanken liegen dürfte. Gerade der Anteil englischsprachiger Publikationen lässt sich aufgrund der zur Verfügung stehenden Daten kaum belastbar abschätzen und auch die Belastbarkeit der Daten zur Verteilung von Publikationsformaten ist zumindest nicht gesichert. Relativ stabil zeichnet sich in den Publikationslisten aus der "FIS Bildung Literaturdatenbank « aber ein Trend hin zu Publikationen mit mehreren Autorinnen und Autoren ab.

Grundsätzlich ist festzustellen, dass Informationsquellen jenseits der Daten des Statistischen Bundesamts, wie sie insbesondere zur Dokumentation von Forschung und Publikationen in der Erziehungswissenschaft erforderlich sind, immer weniger gefördert werden. Sowohl die Einstellung der SOFIS-Datenbank als auch die weniger intensiv betriebene Pflege der FIS-Bildung sind Hinweise darauf, dass Dokumentationsaufgaben großer außeruniversitärer Institute zunehmend zugunsten eigener Forschungen zurückgefahren werden. Dies mag aus institutionspolitischer Sicht zielführend sein, für die Forschungslandschaft insgesamt stellt diese Entwicklung jedoch ein Problem dar, da die in diesen Datenbanken gesammelten Daten selbst wieder Grundlage für zahlreiche wissenschaftliche Analysen darstellen. Hier wäre von Seiten der Wissenschaftspolitik über neue Strategien zur Stärkung der Forschungsdokumentation nachzudenken, da die darüber gewonnenen Erkenntnisse nicht nur der Selbstvergewisserung einzelner Fächer dienlich sind, sondern in einem zunehmend unübersichtlichen und kompetitiven Wettbewerb um Mittel ein gewisses Maß an Transparenz herzustellen vermögen.

\section{Literatur}

Christensen, Marit/Dyrstad, Jan Morten/Innstrand, Siw Tone (2018): Academic work engagement, resources and productivity. Empirical evidence with policy implications. In: Studies in higher education, online first. https://doi.org/10.1080/03075079 .2018.1517304 [Zugriff: 2.10.2019]. 
DFG (=Deutsche Forschungsgemeinschaft) (2018): Förderatlas 2018. Kennzahlen zur öffentlich finanzierten Forschung in Deutschland. Weinheim: Wiley-VCH.

Deutsche Gesellschaft für Soziologie (2012): Wissenschaftliche Evaluation ja - CHERanking nein. Stellungnahme der Deutschen Gesellschaft für Soziologie. In: Erziehungswissenschaft 23, 45, S. 26-29.

Dilger, Alexander (2018): Vor- und Nachteile verschiedener Arten von Drittmitteln. In: Hochschulmanagement 13, 1, S. 2-5.

Enders, Jürgen/Teichler, Ulrich (1997): A victim of their own success? Employment and working conditions of academic staff in comparative perspective. In: Higher education 34, 3, S. 347-372.

Gralka, Sabine/Wohlrabe, Klaus/Bornmann, Lutz (2018): Zur Messung von Forschungsleistungen in der Effizienzanalyse. Drittmittel versus Publikationen. In: Ifo-Schnelldienst 71, 16, S. 26-30.

Kraul, Margret/Schulzeck, Ursula/Weishaupt, Horst (2004): Forschung und wissenschaftlicher Nachwuchs. In: DGfE: Datenreport Erziehungswissenschaft 2004. Hrsg. v. Tippelt, R./Rauschenbach, T./Weishaupt, H. Schriften der Deutschen Gesellschaft für Erziehungswissenschaft. Wiesbaden: VS Verlag für Sozialwissenschaften, S. 91-120.

Röbken, Heinke (2014): Wie verändern sich wissenschaftliche Publikationsaktivitäten im Laufe einer akademischen Karriere? Eine empirische Analyse am Fallbeispiel der Erziehungswissenschaft. In: Die Hochschule: Journal für Wissenschaft und Bildung 23, 1, S. 190-203.

Schmidt, Bernhard/Weishaupt, Horst (2008): Forschung und wissenschaftlicher Nachwuchs. In: DGfE: Datenreport Erziehungswissenschaft 2008. Hrsg. v. Tillmann, K.-J./ Rauschenbach, T./Tippelt, R./Weishaupt, H. Schriften der Deutschen Gesellschaft für Erziehungswissenschaft. Opladen u.a.: Verlag Barbara Budrich, S. 113-138.

Schmidt-Hertha, Bernhard/Tafertshofer, Lorenz/Tippelt, Rudolf (2016): Forschung und Publikationskulturen. In: DGfE: Datenreport Erziehungswissenschaft 2016. Hrsg. v. Koller, H.-C./Faulstich-Wieland, H./Weishaupt, H./Züchner, I. Schriften der Deutschen Gesellschaft für Erziehungswissenschaft. Opladen u.a.: Verlag Barbara Budrich, S. 159-178.

Schmidt-Hertha, Bernhard (2012): Forschung und Publikationskulturen. In: DGfE: Datenreport Erziehungswissenschaft 2012. Hrsg. v. Thole, W./Faulstich-Wieland, H./ Horn, K.-P./Weishaupt, H./Züchner, I. Schriften der Deutschen Gesellschaft für Erziehungswissenschaft. Opladen u.a.: Verlag Barbara Budrich, S. 159-180.

Tafertshofer, Lorenz/Werner, Eva-Maria/Schmidt-Hertha, Bernhard (2018): Grundlagen der Reputation von Studienstandorten: Bewertungsmaßstäbe für die Qualität von Hochschulstandorten und Studiengängen aus der Sicht von sozialwissenschaftlichen Professorinnen und Professoren. In: Beiträge zur Hochschulforschung 2018 2, S. 68-88.

Torka, Marc (2018): Folgen der Drittmittelfinanzierung für Form und Inhalt der Forschung. In: Hochschulmanagement 13, 1, S. 6-12.

Weishaupt, Horst/Merkens, Hans (2000): Forschung und wissenschaftlicher Nachwuchs. In: DGfE: Datenreport Erziehungswissenschaft. Befunde und Materialien 
zur Lage und Entwicklung des Faches in der Bundesrepublik. Hrsg. v. Otto, H.-U./ Krüger, H.-H./Merkens, H./Rauschenbach, T./Schenk, B./Weishaupt, H./Zedler, P. Schriften der Deutschen Gesellschaft für Erziehungswissenschaft. Opladen: Leske + Budrich, S. 117-134.

Wilkesmann, Uwe (2016): Wettbewerb und Hierarchie versus Markt- und WahrheitFetisch - Allokationsmechanismen in der (Erziehungs-)Wissenschaft. In: Erziehungswissenschaft 27, 53, S. 43-51.

Wissenschaftsrat (2013): Empfehlungen zur Zukunft des Forschungsratings. Köln: Wissenschaftsrat. 


\section{Habilitationen und Promotionen in der Erziehungswissenschaft}

Renate Martini ${ }^{1}$

Abgeschlossene Habilitationen und Promotionen sind wichtige Indikatoren wie auch eine wesentliche Grundlage für die Entwicklung einer Wissenschaftsdisziplin. Zugleich bilden sie eine Basis für die kontinuierliche Qualitätssicherung in der Forschung. Daher untersucht dieses Kapitel die Habilitationen und Promotionen separat von anderen Indikatoren zur Beschreibung von Forschungsstärke einer sozialwissenschaftlichen Disziplin oder Einrichtung. Gleichwohl steht der Indikator Abschluss von Habilitationen und Promotionen in Wechselbeziehung zu anderen Indikatoren wie Personalsituation (vgl. Kapitel 4) und Drittmittelakquise (vgl. Kapitel 5).

Erziehungswissenschaftliche Institute und Fachbereiche werden jährlich gebeten, die an ihren Einrichtungen abgeschlossenen Habilitationen und Promotionen an die Zeitschrift für Pädagogik (ZfPäd) zu melden. Mit zahlreichen Informationen (u.a. Thema, Einrichtung, Sprache) lassen sie differenziertere Analysen zu, als es bei Angaben aus statistischen Ämtern möglich wäre. Daher steht zunächst die Auswertung dieses Datenkorpus im Vordergrund. Anschließend wird der Frage nachgegangen, wie sich die Erziehungswissenschaft im Vergleich zu den Fächern Psychologie, Politikwissenschaften, Sozialwissenschaften sowie Wirtschaftswissenschaften entwickelt. Diese Gegenüberstellung wird auf der Basis der Hochschulstatistik des Statistischen Bundesamts ${ }^{2}$ vorgenommen.

Weil dieser Datenreport kein eigenes genderspezifisches Kapitel beinhaltet, wird hier der Anteil von Frauen und Männern bei den einzelnen Aspekten besonders berücksichtigt.

Zum zwanzigjährigen Bestehen des Datenreports Erziehungswissenschaft kann auf eine große Zeitreihe zurückgegriffen werden, die Entwicklungen und Trends noch deutlicher hervortreten lässt als zeitlich segmentierte Abschnitte. Eine jährliche Darstellung seit dem Jahr 2000 lässt sich allein aufgrund des Umfangs nicht realisieren, dennoch zeigen auch die beispielhaft ausgewählten Zeitpunkte Veränderungen auf.

1 Für wichtige Hinweise danke ich Horst Weishaupt, Hermann Avenarius sowie Johannes Hiebl.

2 In Anlehnung an die Bezeichnung der Fächergruppen beim Statistischen Bundesamt. Unter Sozialwissenschaften werden dort Sozialkunde, Sozialwissenschaft und Soziologie zusammengefasst. 


\subsection{Habilitationen und Promotionen in der Zeitschrift für Pädagogik}

Mit der Meldung von Habilitationen und Promotionen aus erziehungswissenschaftlichen Instituten und Fachbereichen an die Zeitschrift für Pädagogik (ZfPäd) steht ein Datenkorpus zur Verfügung, das neben der Häufigkeit der jährlichen Abschlüsse und der Geschlechterverteilung auch Aussagen zu Sprache, inhaltlicher Schwerpunktsetzung wie auch der Entwicklung an einzelnen Hochschulen zulässt. Das wird nachfolgend konkretisiert.

\subsubsection{Analysebasis und Methodik}

Im Anschluss an den letzten Datenreport, der die Habilitationen und Promotionen aus den Jahren 2009 bis 2014 in den Blick genommen hatte, stellen diese Analysen die in den Jahren 2015 bis 2018 an deutschen Hochschulen abgeschlossenen Arbeiten in den Vordergrund. Für die Darstellung von Entwicklungen werden auch die Meldungen für die Jahre 1995, 2000, 2005 sowie 2010 in die Abbildungen einbezogen. Um Trends noch besser erkennen zu können, fließen auch die Werte für den vergleichbaren Zeitraum 2011 bis 2014 bzw. ab 2000 durchgängig in die Diskussion ein.

Bei der Interpretation der Zahlen sind u. a. folgende Aspekte in Betracht zu ziehen (siehe auch Kauder 2012: 735ff.):

a) Nichtberücksichtigung von Qualifizierungsarbeiten: Fachdidaktische Habilitationen und Promotionen werden ebenso wie Arbeiten in der Pädagogischen Psychologie häufig in der Fachdisziplin angefertigt und von Prüfungskommissionen eben dieser Disziplinen abgenommen, sodass sie den erziehungswissenschaftlichen Fakultäten und Instituten nicht bekannt sind. Daraus folgt, dass sie nur partiell, in Abhängigkeit von der Hochschulstruktur in die Statistiken der ZfPäd einfließen.

b) Datenschutz: Alljährlich begründen immer wieder neue Einrichtungen den bewussten Verzicht auf Meldungen an die Zeitschrift für Pädagogik mit datenschutzrechtlichen Bedenken. Bei der Angabe von Habilitand/Habilitandin, Doktorand/Doktorandin, bearbeitetem Thema sowie Gutachterinnen und Gutachtern, wie es in der ZfPäd erfolgt, handelt es sich allerdings nicht um Daten, die einem besonderen Schutz unterliegen. Im Übrigen sind derartige Angaben über Bibliothekskataloge und Fachdatenbanken allgemein zugänglich. Zudem ist die Pflicht zu publizieren bei Dissertationen ja von Rechts wegen geregelt.

c) Korrektur von Angaben: Sind Arbeiten durch Standortwechsel der Betreuer innerhalb eines Jahres mehrfach gemeldet worden oder wurden Arbeiten in aufeinanderfolgenden Jahren wiederholt genannt, werden auf der Basis 
der Angaben in der Deutschen Nationalbibliothek oder auch persönlicher Nachfragen entsprechende Korrekturen vorgenommen. Daher sind die hier verwendeten Werte nicht identisch mit Zählungen innerhalb der ZfPäd. Nachmeldungen werden dem gemeldeten Abschlussjahr zugeordnet.

d) Inhaltsanalyse: Die Inhaltsanalyse für die Habilitationen und Promotionen der Jahre 2009 bis 2014 wurde auf der Basis der in der »FIS Bildung Literaturdatenbank « vorhandenen Verschlagwortung und deren fachsystematischer Zuordnung (vgl. Martini/Schmidt-Hertha 2012: 188ff.) vorgenommen. Da bereits im letzten Datenreport die Treffsicherheit bei der Zuordnung mit dieser Methode punktuell nicht gegeben war, werden die Arbeiten für diesen Datenreport individuell einem inhaltlichen Schwerpunkt, bezeichnet mit Forschungsfeld, zugeordnet. Grundlage für die Entscheidung sind der Titel, die Schlagwörter, das Abstract und ggf. das Inhaltsverzeichnis der einzelnen Arbeit. Die Sektionen der DGfE bilden die Basis für die inhaltliche Orientierung bei der Klassifizierung. Arbeiten, die mehrere Forschungsfelder thematisieren oder nicht eindeutig zugeordnet werden können, werden der Rubrik »Mehrfachzuordnung« zugeführt. Um Entwicklungstendenzen über einen längeren Zeitraum erkennen zu können, werden in diese Clusterung auch die gemeldeten Habilitationen und Promotionen für die Jahre 1995, 2000, 2005 und 2010 einbezogen.

\subsubsection{Erziehungswissenschaftliche Habilitationen}

Die für den Untersuchungszeitraum 2015 bis 2018 an die Zeitschrift für Pädagogik gemeldeten Habilitationen schwanken zwischen 24 und 30 Arbeiten jährlich. Die 107 Habilitationen, die zwischen 2015 und 2018 an deutschen Hochschulen zum Abschluss kamen, werden von 46 Hochschuleinrichtungen an die ZfPäd gemeldet. Das sind acht Habilitationen mehr als im Vergleichszeitraum 2011-2014. An nur vier Universitäten kommen über diesen Zeitraum zwischen fünf und acht Verfahren zum Abschluss. Alle anderen Hochschulen liegen darunter.

Insgesamt verstärkt sich der Trend, dass sich nach der Einführung der Juniorprofessur 2002 die Zahl der Habilitationen etwa um ein Drittel reduziert. Die Daten aus dem Statistischen Bundesamt bestätigen diese Entwicklung. Allerdings zeigt sich nach den beiden deutlichen Rückgängen in den Jahren 2010 und 2013 ein Ansteigen auf eine durchschnittliche Häufigkeit von 26 Habilitationen. Auch wenn die Zahl der Juniorprofessuren in den letzten Jahren auf 89 angewachsen ist (vgl. Kapitel 4), kann ihre Karriereentwicklung noch nicht hinreichend eingeschätzt werden. ${ }^{3}$ Für die Erziehungswissenschaft wird näher

3 Auch umfangreiche Darstellungen zur Situation des wissenschaftlichen Nachwuchses wie etwa der Bundesbericht Wissenschaftlicher Nachwuchs (BuWiN) geben keine Auskunft darüber. Die Daten zur Erziehungswissenschaft werden dort der Fächergruppe Rechts-, Wirtschafts- und Sozialwissenschaften zugeordnet und lassen somit keine Spezifizierung zu. Auch die Analysen der Deutschen Gesellschaft Juniorprofessur nutzen diese Fächergruppe, sodass auch hier keine Informationen zur Situation in der Erziehungswissenschaft gegeben werden. 
zu untersuchen sein, ob die Juniorprofessur die Habilitation dauerhaft ersetzen oder als eine von mehreren möglichen Alternativen neben der Habilitation verstanden wird. Die unterschiedliche Besoldungshöhe der Juniorprofessuren in den einzelnen Bundesländern wie auch zumeist nicht ruhegehaltsfähige Leistungsbezüge für besondere Leistungen könnten die Entscheidung für diesen Karriereweg beeinflussen (vgl. Preißler 2019).

Abbildung 6.1: Erziehungswissenschaftliche Habilitationen und Anteile von Frauen und Männern 1995, 2000, 2005 und 2010-2018

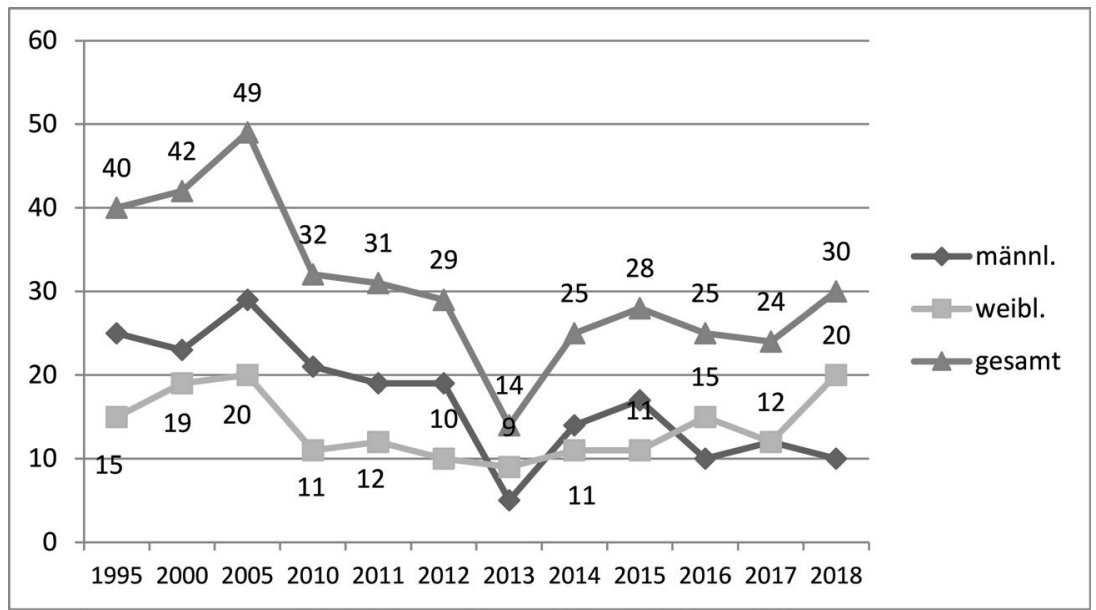

Quelle: Zeitschrift für Pädagogik; eigene Berechnungen

Der Anteil der von Frauen zwischen 2015 und 2018 abgeschlossenen Habilitationsverfahren liegt zwischen $40 \%$ und $60 \%$ und schwankt von Jahr zu Jahr. Bei den 107 abgeschlossenen Habilitationsverfahren sind die Ergebnisse von 5 Arbeiten in englischer Sprache publiziert, 4 davon wurden von Frauen geschrieben. Im Untersuchungszeitraum sind 46 (zuvor 18) kumulative Habilitationen entstanden, die mit 23 (zuvor 8) Frauen und 23 (zuvor 10) Männern nach wie vor nicht auf ein geschlechtsspezifisches Interesse bei der Auswahl dieser Abschlussform deuten.

Hinsichtlich der inhaltlichen Schwerpunktsetzung werden bei den Habilitationen überwiegend Themen aus den Forschungsfeldern Bildungsforschung, Lernen, Fachdidaktik und Bildungstheorie aufgegriffen. Aufgrund der kleinen Fallzahlen lassen sich keine Trends erkennen. Allerdings zeigen die Daten für die Bildungsforschung seit dem Jahr 2000 einen Anstieg bis 2005 auf 15 Arbeiten, dem dann ein steter Rückgang bis auf 4 Habilitationen in 2018 folgt. Für ein Fünftel der Habilitationen konnte aufgrund einer thematischen Vielfalt keine eindeutige Zuordnung vorgenommen werden. In 
den zehn identifizierten Forschungsfeldern ist in fünf dieser Themenfelder der Anteil der Frauen größer als der der Männer.

Tabelle 6.1: Verteilung der Habilitationen nach Forschungsfeldern 2015-2018 mit Anteil der Frauen

\begin{tabular}{lcccc} 
& \multicolumn{2}{c}{ Habilitationen } & \multicolumn{2}{c}{ Frauen } \\
Forschungsfeld & absolut & in & absolut & in \\
\hline Berufs- u. Wirtschaftspädagogik & 7 & 6,5 & 4 & 57,1 \\
\hline Berufsbildung & 0 & 0 & 0 & 0 \\
\hline Bildungsforschung & 17 & 15,9 & 10 & 58,8 \\
\hline Bildungspolitik & 1 & 0,9 & 0 & 0 \\
\hline Bildungstheorie & 11 & 10,3 & 5 & 45,4 \\
\hline Fachdidaktik & 12 & 11,2 & 6 & 50,0 \\
\hline Frühe Bildung & 3 & 2,8 & 2 & 66,7 \\
\hline Hochschule/Studium & 1 & 0,9 & 1 & 100,0 \\
\hline Lehrerbildung & 6 & 5,6 & 3 & 50,0 \\
\hline Lernen/Päd. Psychologie & 16 & 14,9 & 11 & 68,7 \\
\hline Schulsystem/Schule & 0 & 7,5 & 0 & 0 \\
\hline Sonderpädagogik & 4 & 19,6 & 2 & 25,0 \\
\hline Sozialpädagogik & 81 & 3,7 & 25,0
\end{tabular}

Quelle: Zeitschrift für Pädagogik; eigene Zuordnungen

\subsubsection{Erziehungswissenschaftliche Promotionen}

Für den Zeitraum 2015 bis 2018 werden insgesamt 1.727 Promotionen von deutschen Hochschulen an die Zeitschrift für Pädagogik gemeldet, das sind 10 Arbeiten weniger als im vergleichbaren Untersuchungszeitraum 2011-2014 (vgl. Martini 2016: 183f). Seit 2016 hat sich die Zahl der Promotionen allerdings um insgesamt 83 Arbeiten verringert. Im Untersuchungszeitraum haben 1.107 Frauen und 585 Männer ihr Promotionsverfahren abgeschlossen. Für 35 Personen war das Geschlecht nicht zu ermitteln.

Offensichtlich gibt es bei den Promotionen in der Erziehungswissenschaft im Rhythmus von etwa vier Jahren eine Wellenbewegung. Das wird insbesondere ab 2010 deutlich, vgl. Abbildung 6.2. Nach einem Rückgang, der zumeist über zwei Jahre verläuft, ist nach dem Tiefstwert in den beiden Folgejahren ein zumindest ebenso großer Zuwachs zu verzeichnen. Rückgang und Anstieg kompensieren sich nahezu. 
Da bei der Personalstärke der Professuren solche Schwankungen ausbleiben, besteht eine mögliche Erklärung darin, dass die Kapazität der betreuenden Professuren ausgeschöpft ist und erst nach Abschluss von Promotionsverfahren neue Betreuungsverhältnisse eingegangen werden können.

Abbildung 6.2: Erziehungswissenschaftliche Promotionen in der ZfPäd und Anteile von Frauen und Männern 1995, 2000, 2005 und 2010-2018

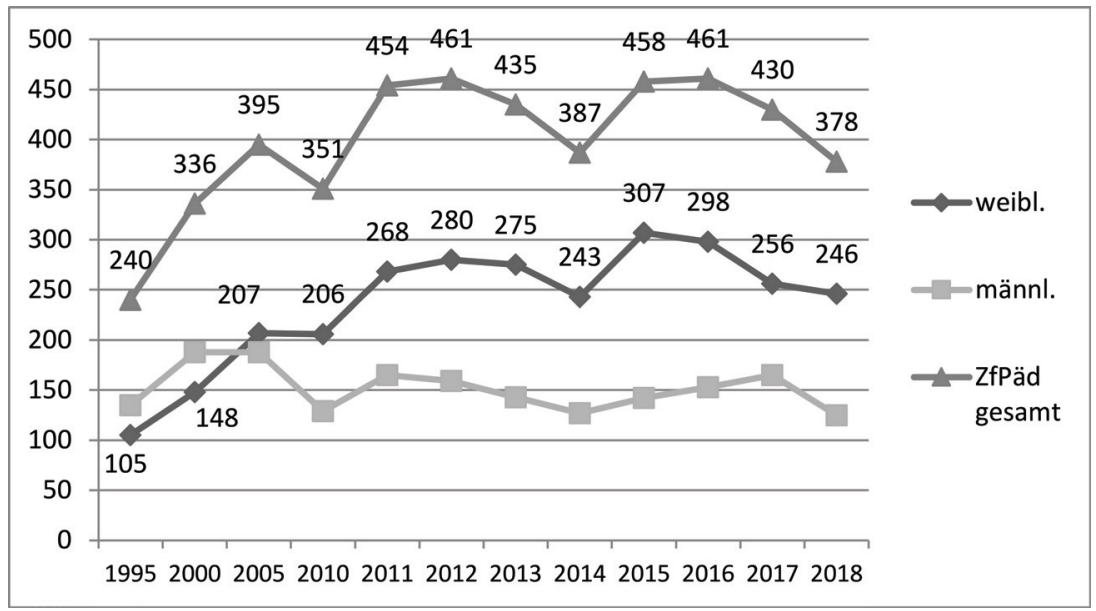

Quelle: Zeitschrift für Pädagogik; eigene Berechnungen

Promovieren bis zum Jahr 2000 mehr Männer als Frauen, so ist nach dem Gleichstand in 2005 ein deutlicher Anstieg des Frauenanteils zu verzeichnen. Ab 2012 schließen nahezu doppelt so viele Frauen wie Männer diese Qualifikationsform ab. Im Jahr 2017 verringert sich dieser Abstand etwas. Im Jahr 2015 erreicht der Anteil 67 Prozentpunkte. In den Folgejahren stabilisiert er sich bei rund $63 \%$. Damit liegt der Frauenanteil bei den Promotionen in der Erziehungswissenschaft 20 Prozentpunkte über dem mittleren Wert aller beim Statistischen Bundesamt dokumentierten Disziplinen.

Die im Zeitraum 2015 bis 2018 abgeschlossenen Promotionen werden von 77 deutschen Hochschulen gemeldet. An 14 Einrichtungen werden mehr als 40 Verfahren in diesem Zeitraum zum Abschluss geführt. Beim letzten Datenreport waren es noch 23 Hochschulen, die im vergleichbaren Zeitraum mehr als 40 Arbeiten abschließen konnten. Nur von der Universität Hamburg werden in dem jetzt untersuchten Zeitraum mehr als 100 Promotionsverfahren gemeldet. Für den Zeitraum 2011 bis 2014 wurden noch fünf Hochschulen mit dieser Häufigkeit genannt.

Über den Analysezeitraum hinweg werden zum Teil erhebliche Veränderungen innerhalb einer Einrichtung deutlich. An 11 Hochschulen ist innerhalb 
der letzten vier Jahre ein Rückgang der Promotionen im Vergleich zu den Jahren 2011 bis 2014 um mehr als 10 Arbeiten zu verzeichnen. An den Universitäten in Bamberg, Erlangen-Nürnberg, Dortmund, Lüneburg, Köln sowie der Freien Universität Berlin hat sich die Anzahl der Promotionen sogar um mehr als 15 verringert. 11 Hochschulen haben in den letzten Jahren aber auch einen Aufwuchs von mehr als 10 Arbeiten erlangen können. In Ludwigsburg fällt dieser Aufwuchs mit 23 Promotionen, in Hamburg mit 29 und in Hildesheim mit 39 Arbeiten besonders deutlich aus.

Für fremdsprachige Publikationen ist bei Promotionen seit dem Jahr 2000 ein sehr verhaltener Anstieg zu beobachten (vgl. Martini/Dees 2012: 48). Für den Untersuchungszeitraum kann ein leichtes, aber kontinuierliches Wachstum auf etwa $9 \%$ konstatiert werden, was immerhin eine Verdopplung seit 2009 bedeutet. Die Freie Universität Berlin (18), die LMU (16) und die Technische Universität (9) - beide in München - sowie die Universität in Oldenburg (8) haben besonders zu dieser Entwicklung beigetragen. In allen Jahren publizieren fast doppelt so viele Frauen wie Männer ihre Untersuchungsergebnisse in englischer Sprache.

Tabelle 6.2: Verteilung der Promotionen nach Forschungsfeldern 2015-2018 mit Anteil der Frauen

\begin{tabular}{lcccc} 
& \multicolumn{2}{c}{ Promotionen } & \multicolumn{2}{c}{ Frauen } \\
Forschungsfeld & absolut & in $\%$ & absolut & in $\%$ \\
Berufs- u. Wirtschaftspädagogik & 115 & 6,6 & 73 & 63,5 \\
\hline Berufsbildung & 42 & 2,4 & 23 & 54,7 \\
\hline Bildungsforschung & 338 & 19,6 & 206 & 60,9 \\
\hline Bildungspolitik & 21 & 1,2 & 10 & 47,6 \\
\hline Bildungstheorie & 58 & 3,4 & 33 & 56,9 \\
\hline Fachdidaktik & 141 & 8,2 & 83 & 58,9 \\
\hline Frühe Bildung & 68 & 3,9 & 54 & 79,4 \\
\hline Hochschule/Studium & 61 & 3,5 & 43 & 70,5 \\
\hline Lehrerbildung & 143 & 8,3 & 95 & 66,4 \\
\hline Lernen/Päd. Psychologie & 182 & 10,3 & 135 & 74,2 \\
\hline Schulsystem/Schule & 44 & 2,5 & 27 & 61,4 \\
\hline Sonderpädagogik & 67 & 3,9 & 115 & 62,7 \\
\hline Sozialpädagogik & 185 & 10,7 & 62,2
\end{tabular}

Quelle: Zeitschrift für Pädagogik; eigene Zuordnungen 
Das kumulative Verfahren zur Publikation der Forschungsergebnisse aus einem Promotionsverfahren hat auch in der Erziehungswissenschaft Fuß gefasst (vgl. auch DGfE 2011). Die ZfPäd hat 2014 dieses Charakteristikum in die Befragung der Hochschulen aufgenommen; es wurden damals 16 publikationsbasierte Promotionen gemeldet. Diese Zahl hat sich 2015 verdoppelt, nahm dann geringfügig ab und ist im Jahr 2018 auf 47 Arbeiten angewachsen. Sie hat sich damit in den letzten vier Jahren fast verdreifacht und macht 7,8 \% aller erziehungswissenschaftlichen Promotionen aus. Die Hochschulen in Dortmund (11), Kiel (18), Ludwigsburg (13), Oldenburg (14), Tübingen (14) sowie die Freie Universität Berlin (10) stützen diese Entwicklung besonders. Auch hier ist der Anteil der Frauen zumeist doppelt so groß wie der der Männer. Berücksichtigt man allerdings die Relation zwischen Frauen und Männern am Gesamtkorpus, dann haben im Abschlussjahr 2017, und nur dann, sowohl bei der Nutzung der englischen Sprache als auch beim kumulativen Abschlussverfahren die Männer den größeren Anteil.

Zur Ermittlung der inhaltlichen Schwerpunkte erziehungswissenschaftlicher Promotionen werden die 1.727 von 2015 bis 2018 an die ZfPäd von deutschen Hochschulen gemeldeten Promotionen ausgewählten Forschungsfeldern zugeordnet. Die Verteilung auf die einzelnen Forschungsfelder sowie der Anteil von Frauen an dem thematischen Schwerpunkt werden in Tabelle 6.2 dargestellt.

Für die Forschungsfelder Bildungsforschung, Sozialpädagogik, Lernen, Fachdidaktik, Lehrerbildung sowie Berufs- und Wirtschaftspädagogik werden die meisten Promotionen gemeldet, wobei sich bei der Lehrerbildung seit 2010 der größte Anstieg zeigt. Das dürfte insbesondere auf die speziellen Förderaktivitäten im BMBF-Rahmenprogramm zur Förderung der empirischen Bildungsforschung sowie die Qualitätsoffensive Lehrerbildung zurückzuführen sein.

In der Bildungsforschung, dem Forschungsfeld mit der größten thematischen Heterogenität lassen sich einige Veränderungen erkennen. Die Bildungsgeschichte macht innerhalb der Bildungsforschung mit $26,7 \%$ zwar noch immer den größten Anteil aus, die Anzahl der abgeschlossenen Promotionen hat sich seit dem Jahr 2000 aber auf ein Drittel reduziert. Fast genauso häufig wie die Bildungsgeschichte werden bildungssoziologische Themen gewählt. Mit nur etwa halb so großer Häufigkeit werden Themen der Biografieforschung, Medienpädagogik, Genderforschung sowie der Wissenschaftsforschung untersucht. Alle anderen Teilbereiche der Bildungsforschung wie etwa die Freizeit- oder Umweltpädagogik werden deutlich seltener in einem Dissertationsthema bearbeitet. 263 Arbeiten lassen sich aufgrund ihrer heterogenen Thematik nicht eindeutig zuordnen.

Obwohl insgesamt deutlich mehr Frauen als Männer promovieren, zeigen deren Anteile innerhalb einzelner Teildisziplinen ein unterschiedliches Bild. In den Themenfeldern Berufsbildung, Bildungspolitik, Bildungstheorie und Fachdidaktik zeigt sich ein annähernd ausgewogenes Verhältnis der Geschlechter. 
Wohingegen in den Forschungsfeldern frühkindliche Bildung, Hochschule/ Studium sowie Lernen/Pädagogische Psychologie mit einem Anteil von nahezu zwei Drittel eine deutlich stärkere Repräsentanz der Frauen vorliegt. Eine »Männerdomäne« bei der Themenwahl lässt sich nicht identifizieren.

Da im nächsten Abschnitt die Daten des Statistischen Bundesamts herangezogen werden, muss an dieser Stelle auf die Unterschiede zwischen beiden Datenquellen eingegangen werden. Auf der Basis des Hochschulstatistikgesetzes bekommt das Statistische Bundesamt die Daten für die Promovierendenstatistik über die Statistischen Landesämter von den Prüfungsämtern der jeweiligen Hochschulen gemeldet (siehe auch Vollmar 2019). Die Daten werden zum 1. Dezember eines jeden Jahres auf der Basis der abgelegten Prüfungen (Disputation) erhoben. Die Redaktion der Zeitschrift für Pädagogik schreibt die erziehungswissenschaftlichen Fachbereiche und Institute der Hochschulen in Deutschland, Österreich und der Schweiz Mitte Januar an und bittet um die Meldung der im vorherigen Kalenderjahr abgeschlossenen Habilitationen und Promotionen. Die Beteiligung aus den einzelnen Hochschulen - wir fokussieren hier Deutschland - ist überaus heterogen. Zahlreiche Hochschulen übermitteln die erbetenen Informationen zügig und im gewünschten Format. Andere Einrichtungen müssen mehrfach erinnert werden, und selbst das ist nicht stets mit einer Rückmeldung verbunden. Daher sind Abweichungen zwischen den Daten des Statistischen Bundesamts und der Zeitschrift für Pädagogik zu erwarten.

Abbildung 6.3: Jährlich ${ }^{1}$ gemeldete Promotionen an die ZfPäd und Daten des Statistischen Bundesamts 1995, 2000-2018

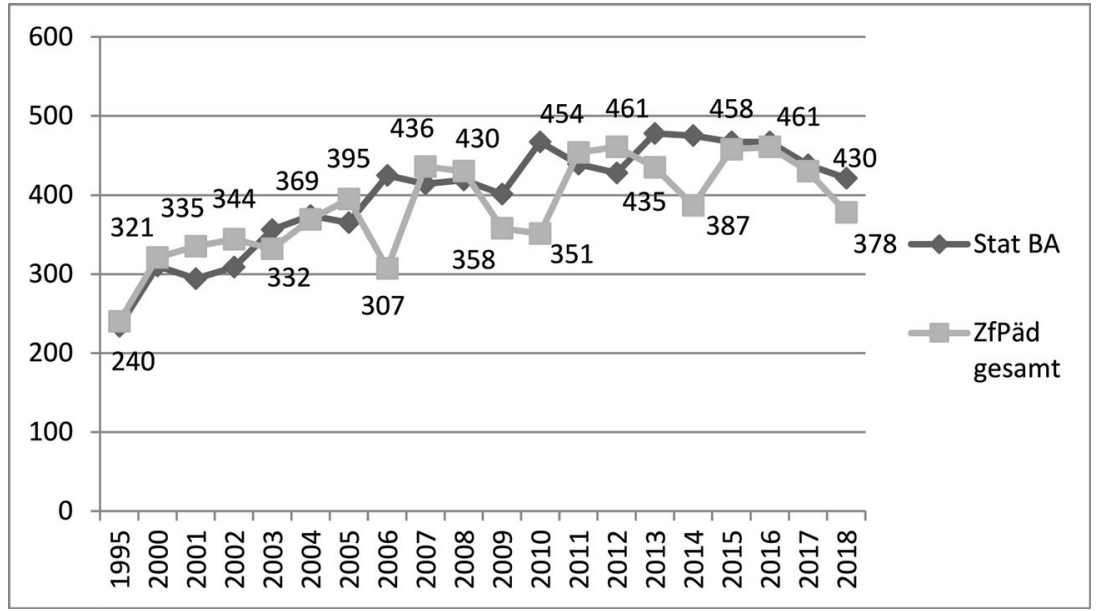

1 Die Zahlen repräsentieren nur die Meldungen an die Zeitschrift für Pädagogik.

Quelle: Statistisches Bundesamt, Fachserie 11, Reihe 4.2; Zeitschrift für Pädagogik 
In der ZfPäd ist seit 2000 - ähnlich dem Statistischen Bundesamt - zunächst ein Anwachsen der Promotionen bis 2005 zu verzeichnen, vgl. Abbildung 6.3. Danach setzen kräftige Schwankungen ein, die sich z.B. nicht durch Veränderungen in der redaktionellen Zuständigkeit seitens der Zeitschrift begründen lassen. Auch nehmen die Abweichungen zu den Daten des Statistischen Bundesamts deutlich zu. In den Jahren 2006 und 2010 beträgt der Unterschied jeweils mehr als 100 Promotionen, 2014 fast 90 Arbeiten. Die Graphen vermitteln den Eindruck, als würden die Daten die ZfPäd mit einer Verzögerung erreichen. In der ersten Dekade dieses Jahrhunderts liegt diese Verschiebung bei einem, später bei zwei Jahren. Erst in den letzten Jahren gelingt eine aktuelle Datenübermittlung.

Ursache dafür können hochschulinterne Prozesse sein. Allerdings dürfte auch die unterschiedliche Meldepraxis an die ZfPäd nicht ohne Einfluss bleiben. Die eine Hochschule übermittelt die Angaben nach Abschluss der Disputation, eine andere erst bei Vorliegen der Publikation, manche informieren sogar zu beiden Zeitpunkten. Auch wenn das jährliche »Einsammeln« der Habilitationen und Promotionen mit erheblichem Aufwand verbunden ist, so scheint eine stärkere Abstimmung mit den Hochschulen und ggf. eine Erinnerung an vorhandene Absprachen (Meldung nach der Disputation) zur Verbesserung der Datenlage wünschenswert.

\subsection{Promotionen und Habilitationen im Fächervergleich}

Dieser Abschnitt geht der Frage nach, wie sich die Erziehungswissenschaft im Vergleich zu anderen Fachdisziplinen entwickelt hat. Für den Vergleich der Promotionen und Habilitationen aus der Erziehungswissenschaft werden - wie in bisherigen Datenreporten - die Fachdisziplinen Psychologie, Politikwissenschaften, Sozialwissenschaften sowie Wirtschaftswissenschaften herangezogen. Die Datengrundlage bildet die Hochschulstatistik des Statistischen Bundesamts. Nur sie ermöglicht einen fachdisziplinären Bezug, wenngleich nur zur Gesamtzahl der abgeschlossenen Arbeiten sowie zum Geschlechteranteil.

\subsubsection{Promotionen im Fächervergleich}

Aus der Hochschulstatistik des Statistischen Bundesamts (vgl. Tabelle 6.3) wird ersichtlich, dass sich in allen Fachgebieten die Häufigkeit von Promotionen in den Jahren seit 1995 deutlich erhöht hat. Bei den Steigerungsraten werden aber Unterschiede deutlich. In der Psychologie (163\%) und den Sozialwissenschaften (114\%) konnte die Anzahl mehr als verdoppelt werden. In 
der Erziehungswissenschaft wurde eine Steigerung um $80 \%$ erreicht. In den Politik- und Wirtschaftswissenschaften liegt das Wachstum bei 43 \%. Die Erziehungswissenschaft liegt damit im Mittelfeld, allerdings deutlich unter dem Niveau des Anstiegs in der Psychologie. Bedauerlich ist, dass das Fachgebiet Psychologie beim Statistischen Bundesamt auch nach der Neukonzipierung der Fächergruppen keinerlei Untergliederung erfährt, sodass die für die Erziehungswissenschaft interessanten Arbeiten aus der Pädagogischen Psychologie weder kenntlich gemacht noch wahrgenommen werden können. Seit 2016 ist die Anzahl abgeschlossener Promotionen in allen Fachgebieten um 10 bis 15 Prozentpunkte rückläufig.

Tabelle 6.3: Abgeschlossene Promotionen nach Fachgebieten 1995, 2000, 2005, 2010 und 2015-2018

\begin{tabular}{|c|c|c|c|c|c|}
\hline & $\begin{array}{l}\text { Erziehungs- } \\
\text { wissenschaft }^{1}\end{array}$ & Psychologie & $\begin{array}{l}\text { Politik- } \\
\text { wissenschaft }\end{array}$ & $\begin{array}{l}\text { Sozial- } \\
\text { wissenschaft }\end{array}$ & $\begin{array}{l}\text { Wirtschafts- } \\
\text { wissenschaft }\end{array}$ \\
\hline 1995 & 243 & 223 & 188 & 181 & 924 \\
\hline 2000 & 310 & 301 & 262 & 238 & 1.024 \\
\hline 2005 & 365 & 486 & 284 & 278 & 1.208 \\
\hline 2010 & 467 & 509 & 274 & 262 & 1.226 \\
\hline 2015 & 467 & 544 & 303 & 354 & 1.037 \\
\hline 2016 & 467 & 598 & 305 & 375 & 1.450 \\
\hline 2017 & 438 & 589 & 273 & 388 & 1.303 \\
\hline 2018 & 421 & 533 & 257 & 328 & 1.284 \\
\hline \multicolumn{6}{|c|}{ Promotionen je 100 Professuren } \\
\hline 1995 & 22,2 & 42,2 & 68,9 & 35,1 & 69,3 \\
\hline 2000 & 31,6 & 57,4 & 96,7 & 50,1 & 75,5 \\
\hline 2005 & 42,3 & 83,9 & 91,6 & 63,8 & 86,2 \\
\hline 2010 & 50,0 & 88,2 & 86,4 & 57,1 & 72,2 \\
\hline 2015 & 46,5 & 83,0 & 88,1 & 70,4 & 49,9 \\
\hline 2016 & 45,6 & 87,0 & 87,9 & 75,6 & 69,5 \\
\hline 2017 & 42,5 & 84,9 & 76,9 & 76,2 & 60,1 \\
\hline 2018 & 40,3 & 74,6 & 69,5 & 62,8 & 58,5 \\
\hline
\end{tabular}

1 einschließlich Sonderpädagogik und Sozialwesen

Quelle: Statistisches Bundesamt, Fachserie 11, Reihe 4.2 und 4.4; eigene Berechnungen

Seit 2010 wächst die Zahl der Professuren in der Erziehungswissenschaft um insgesamt 23 \%, ähnlich der Entwicklung in der Psychologie (21 \%). Das wirkt sich allerdings nicht auf die Zahl der Promotionen je 100 Professuren aus. Wie Tabelle 6.3 verdeutlicht, liegt die Zahl der Promotionen je Professur weiterhin 
deutlich unter den zum Vergleich herangezogenen Disziplinen. In der Erziehungswissenschaft wird nur alle zwei Jahre ein Promotionsverfahren je Professur abgeschlossenen, bei rückläufiger Tendenz.

Diese Entwicklung verwundert angesichts diverser Förderprogramme zur Unterstützung des wissenschaftlichen Nachwuchses, die seit 2008 eingerichtet wurden. Allerdings lässt die Zunahme der Drittmittel in der Erziehungswissenschaft um 60 \% seit 2010 - im Vergleich zur Psychologie (38 \%), Politik- (34 \%), Sozial- (43\%) und Wirtschaftswissenschaften (26\%) - auch vermuten, dass zahlreiche personelle Kapazitäten an die Einwerbung von Drittmitteln gebunden werden (vgl. Kapitel 5.1). Damit würde dem Indikator Drittmittelakquise eine höhere Priorität als dem der Förderung des wissenschaftlichen Nachwuchses eingeräumt. Weitere Gründe für diese Diskrepanz können aber auch in einer stärkeren Anwendungsorientierung geförderter Projekte, der Zunahme befristeter Stellen (vgl. Kapitel 4.1) oder stärkeren personellen Veränderungen innerhalb einer Hochschule liegen.

Abbildung 6.4: Prozentualer Anteil von Frauen bei Promotionen nach Fachgebiet 1995, 2000, 2005, 2010 und 2015-2018

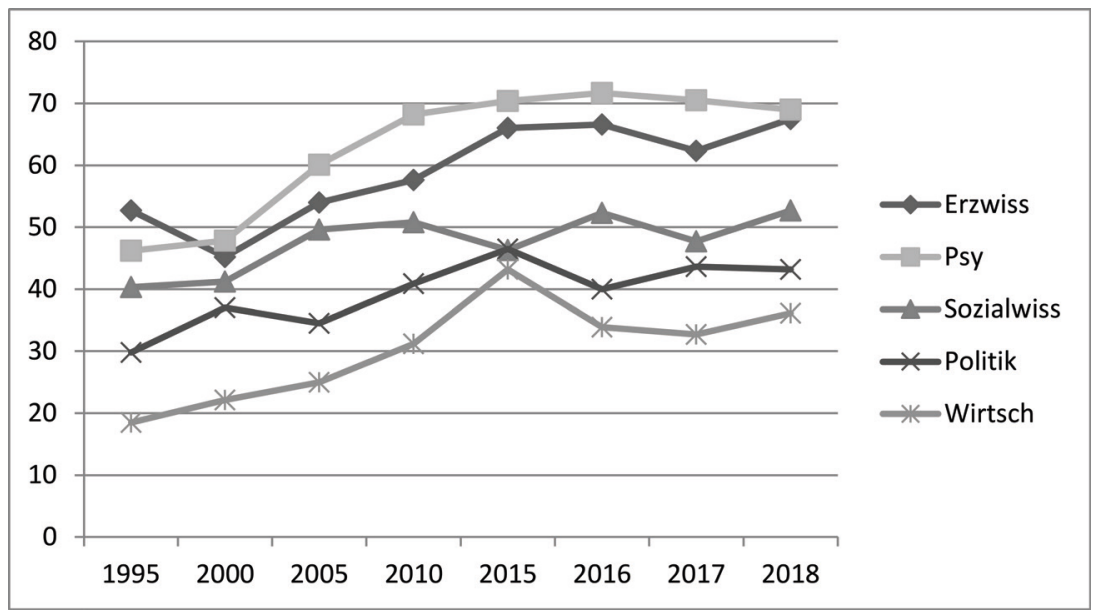

Quelle: Statistisches Bundesamt, Fachserie 11, Reihe 4.2

Der prozentuale Anteil der Frauen bei abgeschlossenen Promotionen nimmt in den einzelnen Fachgebieten unterschiedliche Entwicklungen. Während der Frauenanteil in den Politik- und Wirtschaftswissenschaften über den Zeitverlauf eine ähnliche Entwicklung zeigt, ist er in den Sozialwissenschaften durch Schwankungen charakterisiert. In der Psychologie weist er von 1995 bis 2016 eine stets steigende Tendenz auf, verharrt dann auf demselben Niveau. In der 
Erziehungswissenschaft fällt der Frauenanteil zu Ende der 1990er Jahre um 8 Prozentpunkte ab, um dann bis 2016 um 20 Prozentpunkte anzusteigen. Nach einem leichten Rückgang im Jahr 2017 erreicht er 2018 seinen Höchstwert.

\subsubsection{Habilitationen im Fächervergleich}

Die Entwicklung der Anzahl von Habilitationen seit 1995 erfolgt in den untersuchten Fachgebieten unterschiedlich. Ein Anstieg ist zunächst bei allen Disziplinen zu verzeichnen, was auch durch die Diskussion um die Einführung der Juniorprofessur und damit verbundener unsicherer Karriereplanung bedingt sein kann. Allerdings variiert der Höchstwert der Entwicklung in Zeitpunkt und Umfang zwischen den Fächern. In den Wirtschaftswissenschaften wird er bereits 2000 erreicht. Die anderen Disziplinen folgen jeweils in jährlichem Abstand bis 2004. Danach zeigt sich in allen Fachgebieten trotz einiger Schwankungen eine deutlich rückläufige Entwicklung, vgl. Tabelle 6.4. In der Psychologie sowie den Politikwissenschaften fallen die Zahlen auf das Ausgangsniveau von 1995. In den Wirtschafts- sowie den Sozial- und Erziehungswissenschaften fallen sie sogar darunter.

Tabelle 6.4: Habilitationen nach Fachgebiet 1995, 2000, 2005, 2010 und 20152018

\begin{tabular}{lccccc} 
Jahr & $\begin{array}{c}\text { Erziehungs- } \\
\text { wissenschaft }\end{array}$ & Psychologie & $\begin{array}{l}\text { Politik- } \\
\text { wissenschaft }\end{array}$ & $\begin{array}{l}\text { Sozial- } \\
\text { wissenschaft }\end{array}$ & $\begin{array}{l}\text { Wirtschafts- } \\
\text { wissenschaft }\end{array}$ \\
\hline 1995 & 36 & 41 & 15 & 35 & 51 \\
\hline 2000 & 40 & 47 & 33 & 35 & 116 \\
\hline 2005 & 49 & 56 & 27 & 38 & 85 \\
\hline 2010 & 36 & 43 & 16 & 22 & 49 \\
\hline 2015 & 26 & 42 & 11 & 21 & 54 \\
\hline 2016 & 25 & 47 & 19 & 23 & 43 \\
\hline 2017 & 26 & 33 & 15 & 21 & 49 \\
\hline 2018 & 27 & 41 & 13 & 27 & 45
\end{tabular}

1 einschließlich Sonderpädagogik und Sozialwesen

Quelle: Statistisches Bundesamt, Fachserie 11, Reihe 4.4

Der prozentuale Anteil von Frauen bei Habilitationen weist in den Politik- und Wirtschaftswissenschaften zunächst eine rückläufige Tendenz auf, erlangt dann wie in allen anderen Fachdisziplinen bis 2010 einen steigenden Verlauf, vgl. Abbildung 6.5. Die Psychologie ist das einzige Fachgebiet, in der der Frauenanteil bis 2015/2016 eine kontinuierliche Steigerung verzeichnen kann, wenngleich die Gesamtzahl an Habilitation zurückgeht. Er- 
ziehungs-, Sozial- und Politikwissenschaften weisen hingegen im Jahr 2015 einen erheblichen Rückgang auf, dem in 2016 ein mindestens ebenso großer Anstieg folgt. Nur in den Sozialwissenschaften bleibt der Anteil der Frauen dann stabil. In den Politikwissenschaften geht er seit 2016 stetig zurück und erreicht 2018 nahezu das Niveau von 1995. In der Psychologie sowie in den Erziehungs- und Wirtschaftswissenshaften geht der Frauenanteil bei Habilitationen im Jahr 2017 zurück, um dann in 2018 in ähnlichem Ausmaß wieder anzusteigen. Die Erziehungswissenschaft bekommt 2018 den größten Zuwachs.

Abbildung 6.5: Prozentualer Anteil der Frauen bei Habilitationen nach Fachgebiet 1995, 2000, 2005, 2010 und 2015-2018

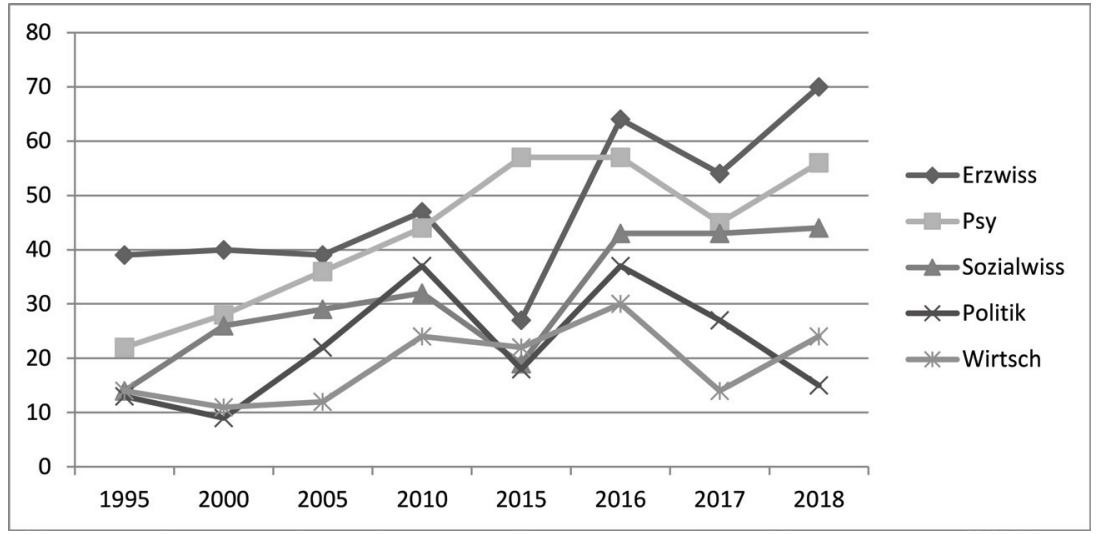

Quelle: Statistisches Bundesamt, Fachserie 11, Reihe 4.4.

\subsection{Fazit}

Die an erziehungswissenschaftlichen Fachbereichen deutscher Hochschulen abgeschlossene Zahl von Promotionen hat sich im Untersuchungszeitraum 2015-2018 im Vergleich zu 2011-2014 kaum verändert, weist aber für die letzten zwei Jahre einen nennenswerten Rückgang auf. Diese Entwicklung folgt allerdings den Zyklen vergangener Jahre. Im Untersuchungszeitraum sind an einzelnen Hochschulen die Veränderungen zu den Jahren 2011-2014 zum Teil erheblich: Während innerhalb von vier Jahren an einigen Einrichtungen ein beachtlicher Anstieg von bis zu 39 Promotionen erreicht wird, zeigen andere Hochschulen Rückgänge von bis zu 25 Arbeiten. Zur Spezifizierung wären umfangreichere Darstellungen und vertiefte Analysen erforderlich. Die unter- 
schiedliche Dynamik der Forschungsentwicklung zwischen den Hochschulstandorten wird daraus dennoch deutlich. Ein Grund für diese großen Schwankungen liegt aber auch im Auslaufen des umfangreichen Förderprogramms für den wissenschaftlichen Nachwuchs im Rahmenprogramm empirische Bildungsforschung, das erst ab 2020 eine Weiterführung erfährt.

Thematisch wird sich bei den Promotionen insbesondere der Bildungsforschung, der Sozialpädagogik, der Berufs- und Wirtschaftspädagogik sowie unterrichtsbezogenen wie auch lernspezifischen Fragestellungen zugewandt. Themen der Lehrausbildung wie auch des Lehrerberufs haben den größten Zuwachs gegenüber dem vorherigen Untersuchungszeitraum genommen. Auch die Zahl der Promotionen im frühkindlichen Bereich ist in den letzten Jahren deutlich angewachsen. Die Häufigkeit sonderpädagogischer Themen hat sich auch durch die Bearbeitung inklusiver Fragestellungen nicht wesentlich verändert. Bildungshistorisch fokussierte Promotionen nehmen deutlich ab. Die hohe Anzahl von Mehrfachzuordnungen deutet darauf hin, dass viele Themen aus einer multidisziplinären Perspektive bearbeitet werden.

Für Habilitationen ist ein deutlicher Rückgang festzustellen. Wenngleich die Zahl der Juniorprofessuren in der Erziehungswissenschaft steigt, lässt eine geringe bzw. unspezifische Datenlage noch keine gesicherten Aussagen zu diesem Karriereweg zu. Bei den Habilitationen werden überwiegend Themen aus den Forschungsfeldern Bildungsforschung, Lernen, Fachdidaktik und Bildungstheorie aufgegriffen. Aufgrund der kleinen Fallzahlen lassen sich keine Trends erkennen.

Insgesamt zeigen sich für die Erziehungswissenschaft bei der Förderung des wissenschaftlichen Nachwuchses im Vergleich zu den anderen in die Auswertung einbezogenen Fächern im Untersuchungszeitraum nur beim Frauenanteil sowie bei der Anzahl von Promotionen je 100 Professuren nennenswerte Unterschiede. Der Anteil von Frauen fällt sowohl bei den Promotionen wie auch bei den Habilitationen in der Erziehungswissenschaft deutlich höher aus als in den Politik-, Sozial- oder Wirtschaftswissenschaften. Auffällig ist der seit 2010 deutlich größere Drittmittelzuwachs in der Erziehungswissenschaft (60 \%) im Vergleich zur Psychologie (38 \%), Politik- (34\%), Sozial- (43\%) und Wirtschaftswissenschaften (26\%), der sich nicht in einer steigenden Zahl erfolgreich abgeschlossener Promotionsverfahren je Professur niederschlägt. Ein Grund dafür kann die personalintensive Einwerbung von Drittmitteln sein. Personal, das dann nicht mehr in ausreichendem Maße für die Betreuung des wissenschaftlichen Nachwuchses zur Verfügung stehen kann.

Für den Vergleich von Daten aus dem Statistischen Bundesamt mit den an die ZfPäd gemeldeten Habilitationen und Promotionen ist die grundlegend verschiedene Erhebungspraxis - Hochschulstatistikgesetz versus »Goodwill« - zu berücksichtigen. Da die Zeitschrift für Pädagogik eine der wenigen deutschen erziehungswissenschaftlichen Fachzeitschriften ist, die stark in der 
Fachcommunity wie auch internal, z.B. über den Social Science Citation Index, wahrgenommen wird, sollte eine aktuelle Darstellung der Ergebnisse aus Habilitations- und Promotionsverfahren aus den Hochschulen im Interesse aller liegen.

\section{Literatur}

DGfE (= Deutsche Gesellschaft für Erziehungswissenschaft) (2011): Publikationsbasierte Dissertationen in der Erziehungswissenschaft - eine Stellungnahme. http://www. dgfe.de/fileadmin/OrdnerRedakteure/Stellungnahmen/2011_Publikationsbasierte_Promotionen.pdf [Zugriff: 31.10.2019]

Kauder, Peter (2012): Die Problematik der der "Zeitschrift für Pädagogik « gemeldeten Promotionen und Habilitationen. In: Zeitschrift für Pädagogik 58, 5, S. 734-756.

Martini, Renate/Schmidt-Hertha, Bernhard (2012): Förderung des wissenschaftlichen Nachwuchses. In:DGfE:DatenreportErziehungswissenschaft2012.Hrsg.v. Thole, W./ Faulstich-Wieland, H./Horn, K.-P./Weishaupt, H./Züchner, I. Schriften der Deutschen Gesellschaft für Erziehungswissenschaft. Opladen u.a.: Verlag Barbara Budrich, S. 181-192.

Martini, Renate/Dees, Werner (2012): Qualifizierungsarbeiten in der Bildungsforschung 1998-2007. In: Weishaupt, H./Rittberger, M. (Hrsg.): Bildungsforschung in Deutschland - eine Situationsanalyse. Bildungsforschung, Bd. 37, S. 43-64 http://www.pedocs. de/volltexte/2013/8206/pdf/Martini_Dees_2012_Qualifizierungsschriften_in_der_ Bildungsforschung_1998_bis_2007.pdf [Zugriff: 31.10.2019]

Martini, Renate (2016): Förderung des wissenschaftlichen Nachwuchses. In: DGfE: Datenreport Erziehungswissenschaft 2016. Hrsg. v. Koller, H.-C./Faulstich-Wieland, H./ Weishaupt, H./Züchner, I. Schriften der Deutschen Gesellschaft für Erziehungswissenschaft. Opladen u.a.: Verlag Barbara Budrich, S. 179-190. https://www.pedocs.de/ frontdoor.php?source_opus=12228 [Zugriff: 31.10.2019]

Preißler, Ulrike (2019): Die Besoldung der Juniorprofessur: W 1 im Überblick beim Bund und den Ländern. In: Forschung und Lehre 26, S. 538-540.

Vollmar, Meike (2019): Neue Promovierendenstatistik: Analyse der ersten Erhebung 2017. In: Wirtschaft und Statistik 1, S. 68-80. 


\title{
7 Der Stellenmarkt von wissenschaftlichem Nachwuchs in Erziehungswissenschaft/Bildungsforschung
}

\author{
Annette Stelter
}

Hat die Methodenausbildung von Nachwuchswissenschaftlerinnen und Nachwuchswissenschaftlern Einfluss auf die wissenschaftliche Laufbahn? Um dieser Frage nachzugehen, wird eine Analyse des akademischen Stellenmarkts im Bereich Erziehungswissenschaft/Bildungsforschung auf Basis von Stellenausschreibungen vorgenommen, die Einblick in die aktuellen Arbeitsmarktanforderungen gibt. ${ }^{1}$ Eine Beschreibung der Anforderungsstruktur im Arbeitsmarkt erlaubt Rückschlüsse auf die Erwartungen, welche an das Qualifikationsprofil und die akademische Ausbildung gestellt werden.

Die Analyse von Stellenausschreibungen ist eine eher selten genutzte, aber aussagefähige Form der Dokumentenanalyse mit einer großen Reichweite. Sie ist in dem Feld der Erziehungs- und Sozialwissenschaft bereits etabliert. Während in 1989 eine Erforschung offener Stellen noch in den Kinderschuhen war (Jackman/Layard/Pissarides 1989), konnte mittlerweile ein tiefergehender Fokus auf Arbeitsinhalte und -profile erreicht werden. So wurde bereits ein inhaltsanalytischer Ansatz zur Analyse von beruflichen Tätigkeiten durchgeführt (Bensberg 2012). Im Folgenden werden zunächst die Rahmenbedingungen der Ausschreibungen (bspw. Dauer, Stellenumfang und disziplinäre Verortung) beschrieben. Des Weiteren werden berufliche Tätigkeiten analysiert, die als analytische und interaktive Nicht-Routine-Tasks (Autor/Levy/Murnane 2003) beschrieben werden können. Insbesondere steht jener Anteil von Tätigkeiten im Fokus, die Fertigkeiten im Bereich der empirischen Forschungsmethoden erfordern. Anhand dieser können Anforderungen des Arbeitsmarkts beschrieben werden, welche dann wiederum Rückschlüsse auf die Erwartungen an die Methodenausbildung erlauben.

\subsection{Methoden}

Um einen Einblick in die aktuelle Arbeitsmarktlage für den wissenschaftlichen Nachwuchs im Bereich von Bildungsforschung und Erziehungswissenschaft zu erlangen, wurden für das Jahr 2017 die Stellenausschreibungen der Univer-

1 Dieser Artikel basiert auf Ergebnissen des vom BMBF geförderten Forschungsprojektes "Systematisierung und Weiterentwicklung der Methodenausbildung und Nachwuchsförderung im Bereich der Bildungsforschung «, das an der Justus-Liebig-Universität Gießen (Leitung Prof. Dr. Ingrid Miethe) durchgeführt wurde (Laufzeit 2016-2019). 
sitäten und Forschungsinstitute gesammelt. Die Ausschreibungen wurden auf den Internetseiten der verschiedenen universitären Fakultäten, Pädagogischen Hochschulen und Forschungseinrichtungen recherchiert und in zweiwöchigen Intervallen immer wieder abgerufen, sodass aufgrund der Dauer von Ausschreibungsfristen (i.d.R. 4 Wochen) die Ausschreibungen weitgehend erfasst werden. Insgesamt können 456 Stellenausschreibungen ausgewertet werden, die an 95 verschiedenen Universitäten, Pädagogischen Hochschulen und Forschungsinstituten ausgeschrieben wurden. Hierbei handelt es sich um keine Vollerhebung (siehe auch Kapitel 4.2). Aus diesen Informationen lassen sich Merkmale der ausgeschriebenen Stelle und forschungsmethodische Schwerpunkte bzw. Arbeitsinhalte extrahieren. Insbesondere werden forschungsrelevante Textabschnitte dokumentiert. Ebenfalls wird vermerkt, ob eine Stelle zur Promotion ausgeschrieben wurde. Die folgenden Analysen werden zum größten Teil auf Basis von deskriptiven Statistiken vorgenommen, die zumeist Verteilungen innerhalb einer Variablen oder bivariate Zusammenhänge beschreiben.

\subsection{Umfang und Inhalt der Stellenausschreibungen}

In einem ersten Schritt werden die formalen Rahmenbedingungen der ausgeschriebenen Stellen beschrieben. Die meisten - knapp vier Fünftel - der akquirierten Stellenausschreibungen bewarben eine Anstellung für wissenschaftliche Mitarbeiterinnen und Mitarbeiter. Weniger als jede zehnte Stelle wird für Lehrkräfte für besondere Aufgaben ausgeschrieben. Dies kann durch eine längere Besetzungsdauer begründet werden. Nur eine von zwanzig Stellen wurde im Bereich der Administration und Koordination ausgeschrieben.

Tabelle 7.1: Geforderte Studienabschlüsse in Stellenausschreibungen (Mehrfachnennungen möglich)

\begin{tabular}{cccccccc}
$\begin{array}{c}\text { Erziehungs- } \\
\text { wissenschaft }\end{array}$ & Lehramt & Psychologie & $\begin{array}{c}\text { Sozial- } \\
\text { wissenschaft }\end{array}$ & Soziologie & $\begin{array}{c}\text { Bildungs- } \\
\text { wissenschaft }\end{array}$ & $\begin{array}{c}\text { Bildungs- } \\
\text { forschung }\end{array}$ & $\begin{array}{c}\text { Soziale } \\
\text { Arbeit }\end{array}$ \\
\hline 256 & 164 & 146 & 105 & 64 & 52 & 30 & 22 \\
\hline $56,1 \%$ & $36,0 \%$ & $32,0 \%$ & $23,0 \%$ & $14,0 \%$ & $11,4 \%$ & $6,6 \%$ & $4,8 \%$
\end{tabular}

Über die Hälfte der Stellen (56,1 \%) werden für Absolventinnen und Absolventen der Erziehungswissenschaft ausgeschrieben (siehe Tabelle 7.1). Ein Lehramtsstudium wird in mehr als einem Drittel der Stellen (36 \%) für die Bewerbung vorausgesetzt. Etwas weniger als ein Drittel der Ausschreibungen (32 \%) setzt ein Psychologiestudium voraus. Ebenfalls relevant sind die Studienfächer Sozialwissenschaft, Soziologie, Bildungswissenschaft und Bildungsforschung. Soziale Arbeit, Wirtschaftswissenschaft, Wirtschaftspädagogik, Medienwissenschaft und Medienpädagogik werden als Studienfach seltener vorausgesetzt. Fast alle Ausschreibungen 
sind fachdisziplinär offen formuliert in dem Sinne, dass Bewerbungen aus angrenzenden Fachdisziplinen ebenfalls möglich seien. Die Kombination von Studienfächern, die für eine Bewerbung vorausgesetzt wird, variiert deutlich. Es zeigt sich keine bevorzugte interdisziplinäre Kombination in den Ausschreibungen. Eine von zehn Ausschreibungen $(\mathrm{n}=44)$ wurde explizit mit der disziplinären Verortung in der Erziehungswissenschaft verfasst, ohne eine weitere Disziplin für eine Bewerbung zuzulassen.

Bei der Analyse werden auch Informationen zur Art des Studienabschlusses berücksichtigt, der für eine Bewerbung gefordert wird. Für fast alle Ausschreibungen werden mehrere Abschlüsse auf einer Qualifikationsebene zugelassen (z.B. Master und Diplom; siehe Abbildung 7.1). Zunächst fällt auf, dass die Anstellungen an den Universitäten und Forschungseinrichtungen sehr selten für Personen zugänglich sind, die einen Bachelorabschluss erworben haben. Für ca. ein Fünftel der ausgeschriebenen Stellen wird das Staatsexamen vorausgesetzt und ebenfalls für ca. ein Fünftel eine Promotion. Die überwiegende Mehrheit der Ausschreibungen setzt einen Master- und/oder Diplomabschluss voraus.

Abbildung 7.1: Geforderter Studienabschluss im Ausschreibungstext der Stellenausschreibungen

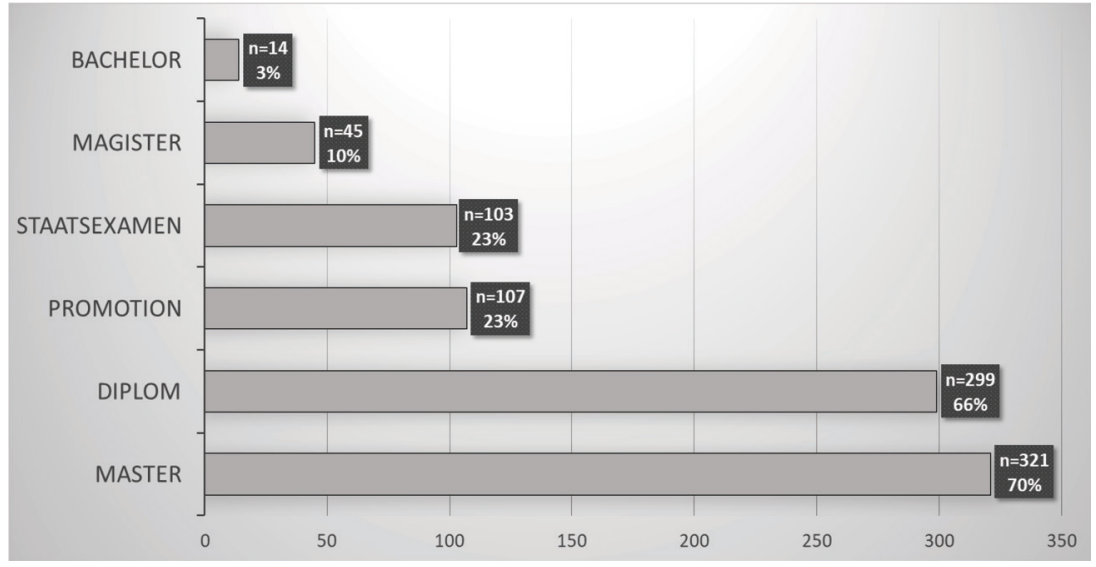

Anmerkung: Weil eine Ausschreibung sich auf mehrere Studienabschlüsse beziehen kann, sind Mehrfachnennungen möglich. Folglich ergibt die Summe der Prozente nicht 100.

Bezogen auf den Zeitraum der Ausschreibungen lässt sich feststellen, dass die Stellen zumeist für drei Jahre befristet werden, wobei diese Stellen partiell verlängerbar waren (Modell 3+3 oder 3+2). Da die Angaben zu den Verlängerungsmöglichkeiten sehr uneinheitlich sind, wurden diese nicht separat erhoben. Stellen, die für ein Jahr oder weniger ausgeschrieben werden, sind häufig Elternzeitvertretungen. Nur 25 der 456 Stellen (5 \%) werden unbefristet ausge- 
schrieben. Damit ermöglicht nur ein ausgesprochen geringer Teil der Stellen eine unbefristete Beschäftigung. Die meisten Ausschreibungen sind auf der Gehaltsstufe 13 eingeordnet (zusammen 92,4\%). Auf dieser Stufe werden wissenschaftliche Mitarbeiterinnen und Mitarbeiter eingestellt, die zur Promotion befähigt sind, aber auch bereits Promovierte. Vergleichsweise unterrepräsentiert in den Ausschreibungen sind die Gehaltsgruppen 14 und 15 (zusammen 4,0 \%). Dies spiegelt die Tatsache wider, dass es auf dieser Gehaltsstufe insgesamt eher wenige Stellen gibt. Zusätzlich muss aber auch berücksichtigt werden, dass es auf derartigen Stellen eine geringere Fluktuation von Personal gibt, sodass diese Stellen seltener neu ausgeschrieben werden. Auch die Gehaltsgruppen 9-12 (zusammen 3,6 \%) wurden vergleichsweise selten ausgeschrieben.

Hinsichtlich der anvisierten Arbeitszeit zeigt sich, dass nur ein geringer Anteil der Ausschreibungen $(<1,0 \%)$ einen Arbeitsumfang von unter $50 \%$ umfasst (siehe Tabelle 7.2). Der größte Anteil der Ausschreibungen (43,9\%) sieht einen Arbeitsumfang von $50 \%$ bis $60 \%$ der wöchentlichen Arbeitszeit vor. Etwas weniger häufiger (23,1 \%) sind umfangreichere Teilzeitstellen (65\%-80\%). Eine Vollzeitstelle wird in knapp einem Drittel $(32,1 \%)$ der Stellenausschreibungen angeboten.

Tabelle 7.2: Wöchentliche Arbeitszeit der Stellenausschreibungen

\begin{tabular}{cccc}
$25 \%-45 \%$ & $50 \%-60 \%$ & $65 \%-80 \%$ & $100 \%$ \\
$\mathrm{n}=4$ & $\mathrm{n}=196$ & $\mathrm{n}=103$ & $\mathrm{n}=143$ \\
\hline $0,9 \%$ & $43,9 \%$ & $23,1 \%$ & $32,1 \%$
\end{tabular}

Die Inhalte der Tätigkeitsbeschreibungen weisen explizite Anforderungen an forschungsmethodische Kenntnisse aus. Nur in 71 Stellenausschreibungen (insgesamt 15,6 \%) gibt es keinen Bezug zu Tätigkeiten im Forschungsbereich. Dabei handelt es sich v.a. um Stellen mit administrativen Anforderungen oder Stellen mit einen Schwerpunkt in der Lehre.

In einigen Stellenausschreibungen lässt sich keine konkrete forschungsmethodische Zuordnung vornehmen, da die gewählten Begrifflichkeiten (z.B. Bildungsforschung, Evaluationsforschung, empirische Forschung) keine eindeutige Zuordnung ermöglichen. So können sich hinter diesen Begriffen sowohl qualitative als auch quantitative Methoden verbergen. Qualitative Methoden werden in 146 Stellenausschreibungen (38 \%) benannt, quantitative Methoden in 204 (53,0 \%) aller Stellenausschreibungen. Spezifische Methodenkenntnisse werden deutlich seltener gefordert. In Tabelle 7.3 wird dargestellt, in welchen Kombinationen qualitative und quantitative Forschungsmethoden für Stellen mit einer Tätigkeit in der Forschung erwünscht sind. Für diese Analysen wurden keine Stellen berücksichtigt, die in der Administration oder der Lehre verortet sind. Dabei zeigt sich, dass der geringste Teil der Ausschreibungen ausschließlich qualitative Methoden erwartet (12,5\%). Ein etwas größerer Anteil der Stellenausschreibungen erwartet sowohl qualitative als auch quantitative Methodenkenntnisse $(25,5 \%)$ und noch 
etwas häufiger werden ausschließlich quantitative Methoden gewünscht $(28,1 \%)$. Der weitaus größte Teil ist methodisch nicht eindeutig zuzuordnen (34,0 \%), da die gewählte Begrifflichkeit (s.o.) keine eindeutige Zuordnung zulässt. Tendenziell zeigt sich, dass Kompetenzen in quantitativen Forschungsmethoden in den Ausschreibungen etwas häufiger gefordert werden.

Tabelle 7.3: Kreuztabelle qualitativer und quantitativer Methodenkenntnisse in Stellenausschreibungen

\begin{tabular}{lcccc} 
& \multicolumn{4}{c}{ Werden quantitative Methoden vorausgesetzt? } \\
& & ja & nein & $\Sigma$ \\
\cline { 2 - 4 } $\begin{array}{l}\text { Werden } \\
\text { qualitative Methoden }\end{array}$ & $\mathrm{n}=98$ & $\mathrm{n}=48$ & $\mathrm{n}=146$ \\
vorausgesetzt? & ja & $25,5 \%$ & $12,5 \%$ & $37,9 \%$ \\
\cline { 2 - 5 } & \multirow{2}{*}{ nein } & $\mathrm{n}=108$ & $\mathrm{n}=131$ & $\mathrm{n}=239$ \\
& $28,1 \%$ & $34,0 \%$ & $62,1 \%$ \\
\hline \multirow{2}{*}{$\Sigma$} & $\mathrm{n}=206$ & $\mathrm{n}=179$ & $\mathrm{n}=385$ \\
& & $53,5 \%$ & $46,5 \%$ & $100 \%$
\end{tabular}

Zusammenfassend kann festgehalten werden, dass die meisten Stellenausschreibungen sich in einigen Merkmalen kaum unterscheiden. So variieren Anstellungstitel, Gehaltsstufe und benötigter Qualifikationsgrad häufig nicht. Unterschiede zeigen sich eher bezüglich der Anstellungsdauer oder der wöchentlichen Arbeitszeit. Ebenso unterscheiden sich die forschungsmethodischen Schwerpunkte in der Stellenbeschreibung. Die Mehrheit der Stellen erfordert qualitative oder quantitative Methodenkenntnisse. Dies bedeutet auch, dass Bewerberinnen und Bewerber ohne diese Kenntnisse sich auf einen Großteil der verfügbaren Stellen nicht bewerben können.

\subsection{Weiterqualifikationsmöglichkeiten für wissenschaftlichen Nachwuchs}

Möglichkeiten zur Weiterqualifikation sind zentral für die Gewinnung talentierter Nachwuchswissenschaftler, aber die unterschiedlichen Anstellungsformate lassen nicht immer eine Qualifizierung zu. Wenn eine Stelle zur Promotion ausgeschrieben wird, sollte diese Weiterqualifikationsmöglichkeit in einem Zusammenhang mit Merkmalen der zugrundeliegenden Stelle stehen.

Knapp die Hälfte der ausgeschriebenen Stellen ( $\mathrm{n}=216$; 47,9 \%) wird zur Promotion ausgeschrieben. In einem Sechstel $(n=72 ; 15,8 \%)$ aller Ausschreibungen ist eine Promotion vorausgesetzt. In wenigen Ausschreibungen $(\mathrm{n}=13$; $2,9 \%$ ) wird eine Promotion nicht explizit genannt, sondern allgemein eine 
Weiterqualifikation unterstützt. Für etwa ein Drittel (n=152; 33,4 \%) wird keinerlei Qualifikationsziel genannt.

Tabelle 7.4: Durchschnittliche wöchentliche Arbeitszeit nach Qualifikationsziel

\begin{tabular}{|c|c|c|c|c|}
\hline & Promotionsstelle & $\begin{array}{l}\text { keine } \\
\text { Promotionsstelle }\end{array}$ & $\begin{array}{l}\text { Promotion } \\
\text { vorausgesetzt }\end{array}$ & $\begin{array}{l}\text { andere } \\
\text { Weiterqualifikation }\end{array}$ \\
\hline $\begin{array}{l}\text { durchschnittlicher } \\
\text { Stellenanteil }\end{array}$ & $\mathrm{n}=217$ & $n=143$ & $n=71$ & $n=13$ \\
\hline $\begin{array}{l}\text { bezogen aut } \\
\text { die Vollzeitstelle }\end{array}$ & $64,1 \%$ & $72,1 \%$ & $88,8 \%$ & $60,8 \%$ \\
\hline
\end{tabular}

Promotionsstellen haben mit im Mittel 64,1 \% der wöchentlichen Arbeitszeit einer Vollzeitstelle einen geringeren Beschäftigungsumfang als Stellen ohne Promotionsmöglichkeit (72,1 \%, siehe Tabelle 7.4). Die Stellen für Post-Doktoranden haben mit durchschnittlich 88,8 \% den höchsten Anteil der wöchentlichen Arbeitszeit einer Vollzeitstelle. Stellen, die eine andere Weiterqualifikation ermöglichen als eine Promotion, haben im Mittel den geringsten Stellenumfang, sodass naheliegt, dass es sich um Stellenkonstruktionen handelt, die kurzfristige Einstellungen in Anlehnung an das Hochschulrahmengesetz ermöglichen.

Abbildung 7.2: Verteilung der Arbeitszeit nach Qualifikationszielen

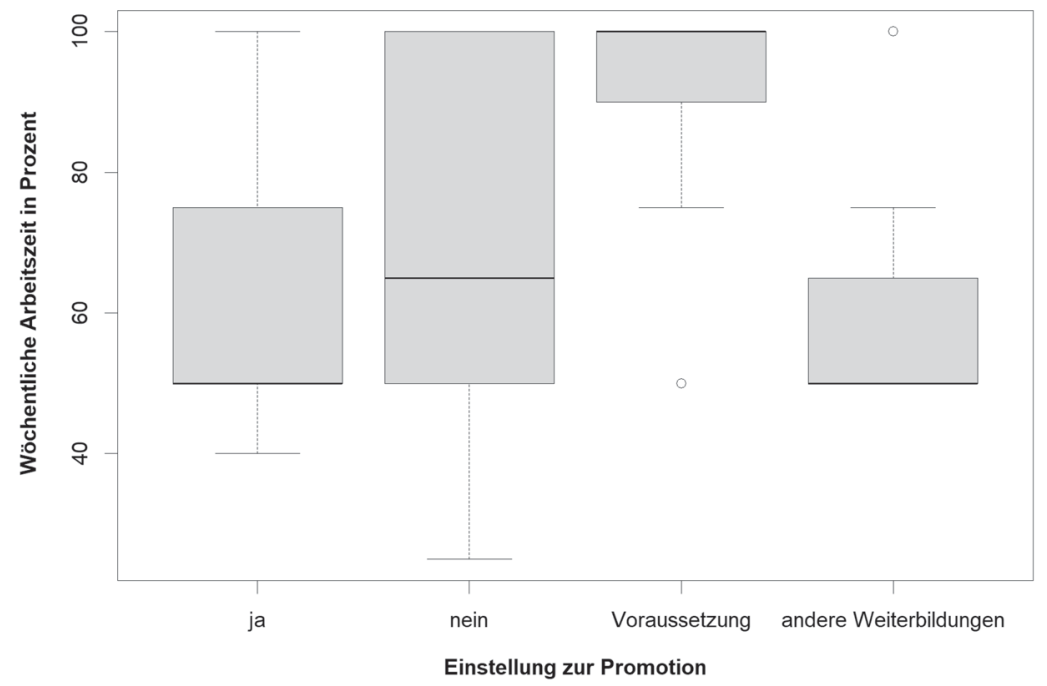

Stellen, die nicht zur Promotion ausgeschrieben wurden, wichen auch häufiger vom mittleren Beschäftigungsumfang ab (siehe Abbildung 7.2). In dieser Grafik 
werden die mittleren Verteilungen der Arbeitszeit dargestellt. In den Boxplots wird in schwarz der Median gekennzeichnet und in den Blöcken ober- und unterhalb die Quantile abgebildet. Stellenausschreibungen ohne Weiterqualifikation wichen deutlich häufiger vom Median ab. Wenn eine Postdoc-Stelle ausgeschrieben wurde, geschah dies häufig als Vollzeitstelle.

In Ausschreibungen von Promotionsstellen, in denen forschungsmethodische Kenntnisse explizit benannt werden, fällt auf, dass quantitative Methodenkompetenz häufiger gefordert wird (siehe Tabelle 7.5). Für 53,7 \% der ausgeschriebenen Promotionsstellen werden quantitative Methodenkenntnisse gefordert, im Gegensatz zu 34,4 \%, die das Wissen über qualitative Methoden voraussetzen. Im Postdoc-Bereich wird der Unterschied noch deutlicher. Für fast die Hälfte (47,2 \%) der Postdoc-Stellen werden quantitative Methodenkenntnisse vorausgesetzt. In nur einem Viertel der Stellen (25\%) wird qualitative Methodenkompetenz gefordert. Die Beschäftigungschancen stellen sich von daher in diesem Bereich für Promovierte mit quantitativen Methodenkenntnissen deutlich besser dar. Dies wird allerdings etwas dadurch relativiert, dass im Bereich "anderer Weiterqualifikationen « die Nachfrage nach qualitativen Methoden etwas höher liegt als die nach quantitativen Methoden. Hierbei handelt es sich allerdings um Stellen mit geringerem Beschäftigungsumfang.

Tabelle 7.5: Vorausgesetzte Kenntnisse der qualitativen Forschungsmethoden

\begin{tabular}{lllll}
$\begin{array}{l}\text { benötigte } \\
\begin{array}{l}\text { Methoden- } \\
\text { kenntnisse }\end{array}\end{array}$ & Promotionsstelle & $\begin{array}{l}\text { keine } \\
\text { Promotionsstelle }\end{array}$ & $\begin{array}{l}\text { Promotion } \\
\text { vorausgesetzt }\end{array}$ & $\begin{array}{l}\text { andere } \\
\text { Weiterqualifikation }\end{array}$ \\
$\begin{array}{l}\text { quantitative } \\
\text { Methoden }\end{array}$ & $\mathrm{n}=117 ; 53,7 \%$ & $\mathrm{n}=52 ; 34,2 \%$ & $\mathrm{n}=34 ; 47,2 \%$ & $\mathrm{n}=3 ; 23,1 \%$ \\
\hline $\begin{array}{l}\text { qualitative Me- } \\
\text { thoden }\end{array}$ & $\mathrm{n}=75 ; 34,4 \%$ & $\mathrm{n}=48 ; 31,6 \%$ & $\mathrm{n}=18 ; 25,0 \%$ & $\mathrm{n}=4 ; 30,8 \%$
\end{tabular}

Zwischen strukturellen und inhaltlichen Charakteristika sowie der Ausschreibung als Promotionsstelle besteht ein Zusammenhang. So stehen die inhaltlichen Anforderungen, genauer die Anforderung quantitative und qualitative Forschungsmethoden zu verwenden, in einem signifikanten Zusammenhang mit der Ausschreibung einer Promotionsstelle.

\subsection{Zusammenfassung und Fazit der Analyse der Stellenausschreibungen}

Ein substanzieller Teil der Stellen, die im Jahr 2017 ausgeschrieben wurden, setzen den Einsatz von Forschungsmethoden voraus. Dies bedeutet, dass Bewerberinnen und Bewerber ohne forschungsmethodische Kenntnisse deutlich 
schlechtere Chancen auf dem akademischen Arbeitsmarkt hatten als solche mit Methodenkompetenzen. Für die forschungsmethodisch qualifizierten Nachwuchswissenschaftlerinnen und Nachwuchswissenschaftler stehen wiederum für diejenigen mit quantitativen Methodenkenntnissen mehr Promotions- und Postdoc-Stellen zur Verfügung als für diejenigen mit qualitativen Kenntnissen.

Die Analysen haben einige Limitationen, die abschließend beschrieben werden müssen. Zum einen handelt es sich bei den Daten um keine Vollerhebung aller relevanten Ausschreibungen. Es wurden zwar angelehnt an Ausschreibungsfristen die Online-Stellenmärkte der Universitäten und Forschungseinrichtungen besucht, dennoch kann nicht ausgeschlossen werden, dass in der Zwischenzeit Ausschreibungen unentdeckt blieben. Auch kann davon ausgegangen werden, dass nicht alle relevanten Stellen offen ausgeschrieben werden, sondern es in der Praxis immer wieder auch zur Vergabe von Stellen unter Ausschreibungsverzicht oder zu internen Stellenausschreibungen kommt. Auch spiegeln die Analysen eine stärkere Gewichtung solcher Stellen, die eine hohe Fluktuation aufweisen: Befristete Stellen werden verständlicherweise häufiger neu ausgeschrieben als unbefristete Stellen. Auch wenn somit keine Aussage über die Stellensituation insgesamt im Bereich Erziehungswissenschaft/Bildungsforschung getroffen werden kann, spiegeln die Ergebnisse doch die aktuelle Ausschreibungs- und damit auch Einstellungspraxis wider. Dies lässt die Aussage zu, dass die hier analysierten Daten durchaus einen verlässlichen Einblick in die aktuellen Ausschreibungen im Bereich der Erziehungswissenschaft und Bildungsforschung an Universitäten und Forschungsinstituten bieten.

In Bezug auf die Methodenausbildung kann festgehalten werden, dass Kenntnisse in den empirischen Forschungsmethoden für eine künftige Anstellung sehr relevant sind. Für Bewerberinnen und Bewerber, die sich für eine wissenschaftliche Karriere entscheiden, sind diese Kenntnisse besonders relevant und können auch über eine weitergehende Karriere, insbesondere die Promotion, entscheiden. In der Folge sollte eine forschungsmethodische Ausbildung Teil des Grundlagenstudiums sein und Vertiefungen ermöglicht werden.

\section{Literatur}

Autor, David H./Levy, Frank/Murnane, Richard J. (2003): The skill content of recent technolological change: An empirical exploration. In: The Quarterly Journal of Economics 118, 4, S. 1279-1333.

Bensberg, Frank (2012): Bildungsbedarfsanalyse auf Grundlage von Stellenanzeigen Potenziale des Text Mining für das Lern-Service-Engineering. In: Mattfeld, D. C./ 
Robra-Bissantz, S.: Multikonferenz Wirtschaftsinformatik 2012. Berlin: Tagungsband der MKWI, S. 425-436.

Jackman, Richard/Layard, Richard/Pissarides, Christopher A. (1989): On vacancies. In: Oxford Bulletin of Economics and Statistics 51, 4, S. 377-394. 



\section{Tabellenanhang}

Tabelle A1: Hauptfach-Bachelorstudiengänge (nach Standorten) 2014/15 und 2018/19 im Vergleich ${ }^{1}$

\begin{tabular}{|c|c|c|c|c|}
\hline Standort ${ }^{2}$ & $\begin{array}{l}\text { Studienfachbezeichnung } \\
2014 / 2015\end{array}$ & $\begin{array}{l}\text { Studienfachbezeichnung } \\
2018 / 2019^{3}\end{array}$ & $\begin{array}{l}\text { Ein- } \\
\text { Fach }\end{array}$ & $\begin{array}{l}\text { Zwei- } \\
\text { Fach }^{4}\end{array}$ \\
\hline Aachen TH & - & - & - & - \\
\hline Augsburg U & EW & $E W$ & $x$ & - \\
\hline Bamberg U & Päd & Päd & - & $x^{* *}$ \\
\hline Bayreuth U & {$[E W]^{5}$} & $E W$ & & $\times$ \\
\hline Berlin FU & EW: B- und EW & $B$ - und EW & $\times$ & - \\
\hline \multirow{2}{*}{ Berlin HU } & EWen & EWen & - & $\times$ \\
\hline & Rehabilitationspädagogik & Rehabilitationspädagogik & $\times$ & - \\
\hline Berlin TU & - & - & - & - \\
\hline Bielefeld U & EW & $E W$ & - & $\times$ \\
\hline Bochum U & EW & $E W$ & - & $\times$ \\
\hline $\begin{array}{l}\text { Braunschweig } \\
\text { TU }\end{array}$ & EW & $E W$ & $\times$ & $x$ \\
\hline Bremen $U$ & - & - & - & - \\
\hline Chemnitz TU & Päd & Päd & $\times$ & - \\
\hline
\end{tabular}

1 Ohne Nebenfächer, weiterbildende/kostenpflichtige Studiengänge und Studiengänge der Gesundheits-, Kunst-, Musik- und Wirtschaftspädagogik. Die Einordnung der Studiengänge in die Ein- bzw. Zwei-Fach-Struktur richtet sich in erster Linie nach den Angaben der Standorte selbst. Ob Studiengänge mit Nebenfächern als Ein-Fach- bzw. Zwei-Fach-Studiengänge angesehen werden, ist in der Eigendefinition der Standorte jedoch sehr unterschiedlich. Wir haben daher Studiengänge mit Nebenfächern von mindestens 30 LP (i.d.R. entspricht dies einem Semester) als Zwei-Fach-Studiengang eingeordnet. Als Hauptfachstudiengang wurde ein Studiengang gezählt, wenn es möglich ist, die Abschlussarbeit in Erziehungswissenschaft zu verfassen.

2 Fett markierte Standorte bieten als generalisiert eingeordnete Bachelorstudiengänge an und kursive Standorte als generalisiert eingeordnete Masterstudiengänge. Fett und kursiv markierte Standorte bieten sowohl im Bachelor- als auch im Masterbereich generalisierte Studiengänge an.

3 Kursiv markierte Studienfachbezeichnungen haben wir als generalisierte und nicht-kursive als spezialisierte Studiengänge eingeordnet.

4 Zahlen in Klammern verweisen auf die Anzahl der Studiengänge, wenn ein Studiengang in mehreren Umfängen angeboten wird.

5 Bei Studiengängen in eckigen Klammern 2014/15 ist es sehr wahrscheinlich, dass es sie schon im WS 2014/15 gab, sie allerdings nicht mitgezählt wurden. 


\begin{tabular}{|c|c|c|c|c|}
\hline Standort ${ }^{2}$ & $\begin{array}{l}\text { Studienfachbezeichnung } \\
2014 / 2015\end{array}$ & $\begin{array}{l}\text { Studienfachbezeichnung } \\
2018 / 2019^{3}\end{array}$ & $\begin{array}{l}\text { Ein- } \\
\text { Fach }\end{array}$ & $\begin{array}{l}\text { Zwei- } \\
\text { Fach }^{4}\end{array}$ \\
\hline Darmstadt TU & Päd & Päd & $x$ & - \\
\hline \multirow{2}{*}{ Dortmund TU } & EW & $E W$ & $x$ & - \\
\hline & Rehabilitationspädagogik & Rehabilitationspädagogik & $x$ & - \\
\hline Dresden TU & $\begin{array}{l}\text { Sozialpädagogik, Sozialarbeit } \\
\text { und Wohlfahrtswissenschaften }\end{array}$ & $\begin{array}{l}\text { Sozialpädagogik, Sozialarbeit } \\
\text { und Wohlfahrtswissenschaften }\end{array}$ & $x$ & - \\
\hline \multirow{2}{*}{$\begin{array}{l}\text { Duisburg- } \\
\text { Essen U }\end{array}$} & EW & $E W$ & $\times$ & - \\
\hline & Soziale Arbeit & Soziale Arbeit & $x$ & - \\
\hline $\begin{array}{l}\text { Eichstätt-Ingol- } \\
\text { stadt KU }\end{array}$ & Päd & -6 & - & - \\
\hline \multirow{3}{*}{ Erfurt U } & EW & $E W$ & - & $x$ \\
\hline & $\begin{array}{l}\text { Primare und elementare } \\
\text { Bildung (Variante Vor- und } \\
\text { Außerschulische Bildung) }\end{array}$ & $\begin{array}{l}\text { Primare und elementare } \\
\text { Bildung (Variante Vor- und } \\
\text { Außerschulische Bildung) }\end{array}$ & - & $x^{*}$ \\
\hline & Förderpädagogik & Förderpädagogik & - & $x^{*}$ \\
\hline $\begin{array}{l}\text { Erlangen-Nürn- } \\
\text { berg U }\end{array}$ & Päd & Päd & - & $x$ \\
\hline Flensburg U & BWen & BWen & - & $x^{*}$ \\
\hline $\begin{array}{l}\text { Frankfurt am } \\
\text { Main U }\end{array}$ & EW & $E W$ & $x$ & - \\
\hline \multirow[b]{2}{*}{ Freiburg PH } & EW & $E W$ & $x$ & - \\
\hline & $\begin{array}{l}\text { Kindheitspädagogik } \\
\text { (7 Semester) }\end{array}$ & $\begin{array}{l}\text { Kindheitspädagogik } \\
\text { (7 Semester) }\end{array}$ & $x$ & - \\
\hline Freiburg U & $\begin{array}{l}\text { BW und } \\
\text { Bildungsmanagement }\end{array}$ & $\begin{array}{l}\text { BW und } \\
\text { Bildungsmanagement }\end{array}$ & - & $x$ \\
\hline \multirow{2}{*}{ Gießen U } & $\begin{array}{l}\text { Bildung und Förderung } \\
\text { in der Kindheit }\end{array}$ & Kindheitspädagogik & $x$ & - \\
\hline & Außerschulische Bildung & $\begin{array}{l}\text { EW mit dem Schwerpkt. } \\
\text { Außerschulische Bildung }\end{array}$ & - & $x^{* *}$ \\
\hline Göttingen U & - & - & - & - \\
\hline Hagen FernU & BW & $B W$ & $x$ & - \\
\hline $\begin{array}{l}\text { Halle-Witten- } \\
\text { berg U }\end{array}$ & EW & $E W$ & $x$ & $x$ \\
\hline Hamburg UBW & B- und EW & B- und EW & $x$ & - \\
\hline Hamburg U & E- und BW & $E$ - und $B W$ & - & $x^{* *}$ \\
\hline HannoverU & Sonderpädagogik & Sonderpädagogik & - & $x^{* *}$ \\
\hline
\end{tabular}

6 Ab dem Wintersemester 2019/2020 wird der BA-Studiengang »Bildungs- und Erziehungswissenschaft« neu eingeführt. 
Tabellenanhang

\begin{tabular}{|c|c|c|c|c|}
\hline Standort ${ }^{2}$ & $\begin{array}{l}\text { Studienfachbezeichnung } \\
2014 / 2015\end{array}$ & $\begin{array}{l}\text { Studienfachbezeichnung } \\
2018 / 2019^{3}\end{array}$ & $\begin{array}{l}\text { Ein- } \\
\text { Fach }\end{array}$ & $\begin{array}{l}\text { Zwei- } \\
\text { Fach }^{4}\end{array}$ \\
\hline Heidelberg PH & $\begin{array}{l}\text { Frühkindliche und } \\
\text { Elementarbildung }\end{array}$ & $\begin{array}{l}\text { Frühkindliche und } \\
\text { Elementarbildung }\end{array}$ & $x$ & - \\
\hline Heidelberg U & BW & $B W$ & - & $\times$ \\
\hline \multirow[b]{2}{*}{ Hildesheim U } & EW & $E W$ & $\times$ & - \\
\hline & $\begin{array}{l}\text { Sozial- und } \\
\text { Organisationspädagogik }\end{array}$ & $\begin{array}{l}\text { Sozial- und } \\
\text { Organisationspädagogik }\end{array}$ & $x$ & - \\
\hline Jena U & EW & $E W$ & - & $\times$ \\
\hline $\begin{array}{l}\text { Karlsruhe KIT } \\
\text { (TH) }\end{array}$ & Päd & Päd & - & $x^{* *}$ \\
\hline Karlsruhe PH & Pädagogik der Kindheit & Pädagogik der Kindheit & $x$ & - \\
\hline Kassel U & Soziale Arbeit & Soziale Arbeit & $\times$ & - \\
\hline Kiel U & Päd & Päd & - & $x$ \\
\hline \multirow{2}{*}{$\begin{array}{l}\text { Koblenz-Landau } \\
U\end{array}$} & Päd + EW + AEW & $P \ddot{d}+E W+A E W$ & $\times(2)^{7}$ & $\times$ \\
\hline & $\begin{array}{l}\text { Betriebspädagogik/ } \\
\text { Personalentwicklung }\end{array}$ & $\begin{array}{l}\text { Betriebspädagogik/ } \\
\text { Personalentwicklung }\end{array}$ & - & $\times$ \\
\hline \multirow{2}{*}{ Köln U } & EW & $E W$ & $\times$ & $\times$ \\
\hline & Frühförderung & Frühförderung & $\times$ & - \\
\hline Leipzig U & - & - & - & - \\
\hline \multirow{3}{*}{$\begin{array}{l}\text { Ludwigsburg } \\
\mathrm{PH}\end{array}$} & $\begin{array}{l}\text { BW/Lebenslanges } \\
\text { Lernen }\end{array}$ & $B W$ & $x$ & - \\
\hline & $\begin{array}{l}\text { Frühkindliche Bildung } \\
\text { und Erziehung }\end{array}$ & $\begin{array}{l}\text { Frühkindliche Bildung } \\
\text { und Erziehung }\end{array}$ & $x$ & - \\
\hline & Kultur/Medienbildung & Kultur- und Medienbildung & $\times$ & - \\
\hline Lüneburg U & - & - & - & - \\
\hline \multirow[b]{2}{*}{ Magdeburg U } & BW & $B W$ & $\times$ & $\times$ \\
\hline & $\begin{array}{l}\text { Medienbildung: } \\
\text { Audiovisuelle Kultur und } \\
\text { Kommunikation }\end{array}$ & $\begin{array}{l}\text { Medienbildung: } \\
\text { Audiovisuelle Kultur und } \\
\text { Kommunikation }\end{array}$ & $x$ & - \\
\hline Mainz U & EW & $E W$ & - & $\times$ \\
\hline Marburg U & E- und BW & $E$ - und BW & $\times$ & - \\
\hline München UBW & $\begin{array}{l}\text { BW, insb. interkulturelle Medien- } \\
\text { und Erwachsenenbildung }\end{array}$ & $\begin{array}{l}\text { BW, insb. interkulturelle Medien- } \\
\text { und Erwachsenenbildung }\end{array}$ & $x$ & - \\
\hline München U & Päd/BW & Päd/BW & - & $\times$ \\
\hline Münster U & EW & $E W$ & $\times$ & - \\
\hline
\end{tabular}

7 Zwei Standorte mit je eigenen Ein-Fach-Studiengängen. 


\begin{tabular}{|c|c|c|c|c|}
\hline Standort ${ }^{2}$ & $\begin{array}{l}\text { Studienfachbezeichnung } \\
2014 / 2015\end{array}$ & $\begin{array}{l}\text { Studienfachbezeichnung } \\
2018 / 2019^{3}\end{array}$ & $\begin{array}{l}\text { Ein- } \\
\text { Fach }\end{array}$ & $\begin{array}{l}\text { Zwei- } \\
\text { Fach }^{4}\end{array}$ \\
\hline \multirow{2}{*}{ Oldenburg U } & Päd & Päd & $\times$ & $\times(2)$ \\
\hline & Sonderpädagogik & Sonderpädagogik & - & $\times(2)^{*}$ \\
\hline OsnabrückU & EW & $E W$ & - & $x^{*}$ \\
\hline Paderborn U & EW & $E W$ & - & $\times$ \\
\hline Potsdam U & EW & $E W$ & - & $\times(2)$ \\
\hline Regensburg U & EW & $E W$ & - & $x^{* *}$ \\
\hline Rostock U & - & - & - & - \\
\hline $\begin{array}{l}\text { Schwäbisch } \\
\text { Gmünd PH }\end{array}$ & $\begin{array}{l}\text { Kindheitspädagogik } \\
\text { (7 Semester) }\end{array}$ & $\begin{array}{l}\text { Kindheitspädagogik } \\
\text { (7 Semester) }\end{array}$ & $x$ & - \\
\hline \multirow{2}{*}{ Siegen U } & Päd: Entwicklung und Inklusion & Päd: Entwicklung und Inklusion & $\times$ & - \\
\hline & Soziale Arbeit & Soziale Arbeit & $\times$ & - \\
\hline Stuttgart U & $\begin{array}{l}\text { Berufspädagogik/ } \\
\text { Technikpädagogik }\end{array}$ & $\begin{array}{l}\text { Berufspädagogik/ } \\
\text { Technikpädagogik }\end{array}$ & - & $x$ \\
\hline Trier U & $\begin{array}{l}\text { EW: Sozial- und } \\
\text { Organisationspädagogik }\end{array}$ & $\begin{array}{l}\text { EW: Sozial- und } \\
\text { Organisationspädagogik }\end{array}$ & $x$ & - \\
\hline Tübingen $U$ & EW & $\begin{array}{l}\text { EW und Soziale Arbeit/ } \\
\text { Erwachsenenbildung }\end{array}$ & $x$ & - \\
\hline \multirow{2}{*}{ Vechta U } & EW & $E W$ & - & $\times$ \\
\hline & Soziale Arbeit & Soziale Arbeit & $\times$ & - \\
\hline \multirow{3}{*}{ Weingarten $\mathrm{PH}$} & Elementarbildung & Elementarbildung & $\times$ & - \\
\hline & $\begin{array}{l}\text { Medien- und } \\
\text { Bildungsmanagement }\end{array}$ & $\begin{array}{l}\text { Medien- und } \\
\text { Bildungsmanagement }\end{array}$ & $\times$ & - \\
\hline & $\begin{array}{l}\text { [Mehrsprachigkeit und } \\
\text { Interkulturelle Bildung] }\end{array}$ & $\begin{array}{l}\text { Mehrsprachigkeit und } \\
\text { Interkulturelle Bildung }\end{array}$ & $x$ & - \\
\hline Wuppertal U & EW & $E W$ & - & $x^{*}$ \\
\hline \multirow{2}{*}{ Würzburg U } & Päd & Päd & - & $\times(2)$ \\
\hline & Sonderpädagogik & Sonderpädagogik & - & $\times$ \\
\hline
\end{tabular}

* polyvalente Studiengänge

** Einfach-Studiengänge mit Nebenfächern $\geq 30$ Leistungspunkten 
Tabelle A2: Hauptfach-Masterstudiengänge (nach Standorten) 2014/15 und 2018/19 im Vergleich ${ }^{1}$

\begin{tabular}{|c|c|c|c|c|}
\hline Standort ${ }^{2}$ & $\begin{array}{l}\text { Studienfachbezeichnung } \\
2014 / 2015\end{array}$ & $\begin{array}{l}\text { Studienfachbezeichnung } \\
2018 / 2019^{3}\end{array}$ & $\begin{array}{l}\text { Ein- } \\
\text { Fach }\end{array}$ & $\begin{array}{l}\text { Zwei- } \\
\text { Fach }^{4}\end{array}$ \\
\hline \multirow{2}{*}{ Aachen TH } & $\begin{array}{l}\text { Bildung- und } \\
\text { Wissensmanagement }\end{array}$ & & - & - \\
\hline & $\begin{array}{l}\text { Empirische } \\
\text { Bildungsforschung }\end{array}$ & $\begin{array}{l}\text { Empirische } \\
\text { Bildungsforschung }\end{array}$ & $x$ & - \\
\hline Augsburg $U$ & $\begin{array}{l}\text { EW mit Schwerpkt. } \\
\text { Heterogenität in } \\
\text { Erziehung und Bildung }\end{array}$ & $\begin{array}{l}\text { EW mit Schwerpkt. } \\
\text { Heterogenität in } \\
\text { Erziehung und Bildung }\end{array}$ & $\times$ & - \\
\hline \multirow{4}{*}{ BambergU } & E- und BW & $E$ - und BW & $\times$ & - \\
\hline & $\begin{array}{l}\text { Erwachsenenbildung/ } \\
\text { Weiterbildung }\end{array}$ & $\begin{array}{l}\text { Erwachsenenbildung/ } \\
\text { Weiterbildung }\end{array}$ & $x$ & - \\
\hline & $\begin{array}{l}\text { Empirische } \\
\text { Bildungsforschung (MSc) }\end{array}$ & $\begin{array}{l}\text { Empirische } \\
\text { Bildungsforschung (MSc) }\end{array}$ & $x$ & - \\
\hline & $\begin{array}{l}\text { Bildungsmanagement und } \\
\text { Schulführung }\end{array}$ & $\begin{array}{l}\text { Bildungsmanagement und } \\
\text { Schulführung }\end{array}$ & $x$ & - \\
\hline Bayreuth U & - & - & - & - \\
\hline Berlin FU & BW & $B W$ & $x$ & - \\
\hline \multirow{3}{*}{ Berlin HU } & EWen & EWen & $x$ & - \\
\hline & $\begin{array}{l}\text { Erwachsenenpädagogik/ } \\
\text { Lebenslanges Lernen }\end{array}$ & $\begin{array}{l}\text { Erwachsenenbildung/ } \\
\text { Lebenslanges Lernen }\end{array}$ & $x$ & - \\
\hline & Rehabilitationspädagogik & Rehabilitationspädagogik & $x$ & - \\
\hline Berlin TU & $\begin{array}{l}\text { BW: Organisation und } \\
\text { Beratung }\end{array}$ & $\begin{array}{l}\text { BW: Organisation und } \\
\text { Beratung }\end{array}$ & $x$ & - \\
\hline
\end{tabular}

1 Ohne Nebenfächer, weiterbildende/kostenpflichtige Studiengänge und Studiengänge der Gesundheits-, Kunst-, Musik- und Wirtschaftspädagogik. Die Einordnung der Studiengänge in die Ein- bzw. Zwei-Fach-Struktur richtet sich in erster Linie nach den Angaben der Standorte selbst. Ob Studiengänge mit Nebenfächern als Ein-Fach- bzw. Zwei-Fach-Studiengänge angesehen werden, ist in der Eigendefinition der Standorte jedoch sehr unterschiedlich. Wir haben daher Studiengänge mit Nebenfächern von mindestens 30 LP (i.d.R. entspricht dies einem Semester) als Zwei-Fach-Studiengang eingeordnet. Als Hauptfachstudiengang wurde ein Studiengang gezählt, wenn es möglich ist, die Abschlussarbeit in Erziehungswissenschaft zu verfassen.

2 Fett markierte Standorte bieten als generalisiert eingeordnete Bachelorstudiengänge an und kursive Standorte als generalisiert eingeordnete Masterstudiengänge. Fett und kursiv markierte Standorte bieten sowohl im Bachelor- als auch im Masterbereich generalisierte Studiengänge an.

3 Kursiv markierte Studienfachbezeichnungen haben wir als generalisierte und nicht-kursive als spezialisierte Studiengänge eingeordnet.

4 Zahlen in Klammern verweisen auf die Anzahl der Studiengänge, wenn ein Studiengang in mehreren Umfängen angeboten wird. 


\begin{tabular}{|c|c|c|c|c|}
\hline Standort ${ }^{2}$ & $\begin{array}{l}\text { Studienfachbezeichnung } \\
2014 / 2015\end{array}$ & $\begin{array}{l}\text { Studienfachbezeichnung } \\
2018 / 2019^{3}\end{array}$ & $\begin{array}{l}\text { Ein- } \\
\text { Fach }\end{array}$ & $\begin{array}{l}\text { Zwei- } \\
\text { Fach }^{4}\end{array}$ \\
\hline Bielefeld U & EW & $E W$ & $\times$ & - \\
\hline Bochum U & EW & $E W$ & $x$ & $\times$ \\
\hline $\begin{array}{l}\text { Braunschweig } \\
\text { TU }\end{array}$ & $\begin{array}{l}\text { Organisation, } \\
\text { Governance, Bildung }\end{array}$ & $\begin{array}{l}\text { Organisation, } \\
\text { Governance, Bildung }\end{array}$ & $x$ & - \\
\hline Bremen $U$ & E- und BWen & E- und BWen & $\times$ & - \\
\hline Chemnitz TU & $\begin{array}{l}\text { Päd mit dem Schwerpkt. } \\
\text { Lernkulturen }\end{array}$ & Päd & $x$ & - \\
\hline Darmstadt TU & $\begin{array}{l}\text { BWen: Bildung in globalen } \\
\text { Technisierungsprozessen }\end{array}$ & $\begin{array}{l}\text { BWen: Bildung in globalen } \\
\text { Technisierungsprozessen }\end{array}$ & $x$ & - \\
\hline \multirow{2}{*}{ Dortmund TU } & EW & $E W$ & $\times$ & - \\
\hline & Rehabilitationspädagogik & Rehabilitationswissenschaften & $\times$ & - \\
\hline \multirow[t]{2}{*}{ Dresden TU } & $\begin{array}{l}\text { Weiterbildungsforschung und } \\
\text { Organisationsentwicklung }\end{array}$ & $\begin{array}{l}\text { Weiterbildungsforschung und } \\
\text { Organisationsentwicklung }\end{array}$ & $\times$ & - \\
\hline & Sozialpädagogik & Sozialpädagogik & $\times$ & - \\
\hline \multirow{2}{*}{$\begin{array}{l}\text { Duisburg- } \\
\text { Essen U }\end{array}$} & $\begin{array}{l}\text { Erwachsenenbildung/ } \\
\text { Weiterbildung }\end{array}$ & $\begin{array}{l}\text { Erwachsenenbildung/ } \\
\text { Weiterbildung }\end{array}$ & $x$ & - \\
\hline & Soziale Arbeit & Soziale Arbeit & $x$ & - \\
\hline \multirow{2}{*}{$\begin{array}{l}\text { Eichstätt- } \\
\text { Ingolstadt KU }\end{array}$} & $\begin{array}{l}\text { Soziale Arbeit } \\
\text { (3 Semester) }\end{array}$ & $\begin{array}{l}\text { Soziale Arbeit } \\
\text { (3 Semester) }\end{array}$ & $x$ & - \\
\hline & - & $\begin{array}{l}\text { Schulsozialarbeit/ } \\
\text { Jugendsozialarbeit an Schulen } \\
\text { (3 Semester) }\end{array}$ & $x$ & - \\
\hline \multirow{2}{*}{ Erfurt U } & $\begin{array}{l}\text { EW - Innovation und Manage- } \\
\text { ment im Bildungswesen }\end{array}$ & $\begin{array}{l}\text { EW - Innovation und Manage- } \\
\text { ment im Bildungswesen }\end{array}$ & $\times$ & - \\
\hline & $\begin{array}{l}\text { Sonder- und } \\
\text { Integrationspädagogik }\end{array}$ & $\begin{array}{l}\text { Sonder- und } \\
\text { Integrationspädagogik }\end{array}$ & $x$ & - \\
\hline \multirow{3}{*}{$\begin{array}{l}\text { Erlangen-Nürn- } \\
\text { berg } U\end{array}$} & Päd & Päd & $x$ & - \\
\hline & $\begin{array}{l}\text { Erziehungswissenschaftlich- } \\
\text { empirische Bildungsforschung }\end{array}$ & $\begin{array}{l}\text { Erziehungswissenschaftlich- } \\
\text { empirische Bildungsforschung }\end{array}$ & $\times$ & - \\
\hline & Theaterpädagogik & - & - & - \\
\hline Flensburg U & $\begin{array}{l}\text { Bildung in Europa - Education } \\
\text { in Europe }\end{array}$ & $\begin{array}{l}\text { EW: Bildung in Europa - Educa- } \\
\text { tion in Europe }\end{array}$ & $x$ & - \\
\hline $\begin{array}{l}\text { Frankfurt am } \\
\text { Main U }\end{array}$ & EW & $E W$ & $x$ & - \\
\hline \multirow[t]{2}{*}{ Freiburg PH } & EW & $\begin{array}{l}\text { EW mit den Schwerpkt. } \\
\text { Erwachsenenbildung } \\
\text { oder Sozialpädagogik }\end{array}$ & $\times$ & - \\
\hline & Medien in der Bildung & - & - & - \\
\hline
\end{tabular}


Tabellenanhang

\begin{tabular}{|c|c|c|c|c|}
\hline Standort ${ }^{2}$ & $\begin{array}{l}\text { Studienfachbezeichnung } \\
2014 / 2015\end{array}$ & $\begin{array}{l}\text { Studienfachbezeichnung } \\
2018 / 2019^{3}\end{array}$ & $\begin{array}{l}\text { Ein- } \\
\text { Fach }\end{array}$ & $\begin{array}{l}\text { Zwei- } \\
\text { Fach }^{4}\end{array}$ \\
\hline Freiburg U & BW: Lehren und Lernen & BW: Lehren und Lernen & $\times$ & - \\
\hline \multirow{2}{*}{ Gießen U } & $\begin{array}{l}\text { EW mit dem Schwerpkt. } \\
\text { Außerschulische Bildung }\end{array}$ & $\begin{array}{l}\text { EW mit dem Schwerpkt. } \\
\text { Außerschulische Bildung }\end{array}$ & - & $x^{* *}$ \\
\hline & $\begin{array}{l}\text { Inklusive Pädagogik und } \\
\text { Elementarbildung }\end{array}$ & $\begin{array}{l}\text { Inklusive Pädagogik und } \\
\text { Elementarbildung }\end{array}$ & $x$ & - \\
\hline Göttingen $U$ & $\begin{array}{l}\text { EW: mit dem Schwerpkt. } \\
\text { Forschung und Entwicklung im } \\
\text { Bildungswesen }\end{array}$ & $E W$ & $\times$ & \\
\hline Hagen FernU & $\begin{array}{l}\text { Bildung und Medien: } \\
\text { eEducation }\end{array}$ & $\begin{array}{l}\text { Bildung und Medien: } \\
\text { eEducation }\end{array}$ & $\times$ & - \\
\hline $\begin{array}{l}\text { Halle-Witten- } \\
\text { berg } U\end{array}$ & $\begin{array}{l}\text { EW: Professionalität \& } \\
\text { Bildungsforschung }\end{array}$ & $\begin{array}{l}\text { EW: Professionalität \& } \\
\text { Bildungsforschung }\end{array}$ & $x$ & - \\
\hline Hamburg UBW & B- und EW & B- und EW & $x$ & - \\
\hline \multirow[b]{2}{*}{ Hamburg $U$} & E- und BW & $E$ - und BW & $\times$ & - \\
\hline & $\begin{array}{l}\text { Mehrsprachigkeit und Bildung: } \\
\text { MOTION - Master of Multi- } \\
\text { lingual Educational Linguistics }\end{array}$ & $\begin{array}{l}\text { Mehrsprachigkeit und Bildung: } \\
\text { MOTION - Master of Multi- } \\
\text { lingual Educational Linguistics }\end{array}$ & $x$ & - \\
\hline \multirow[b]{2}{*}{ Hannover U } & BWen & BWen & $\times$ & - \\
\hline & $\begin{array}{l}\text { Sonderpädagogik und } \\
\text { Rehabilitationswissenschaften }\end{array}$ & $\begin{array}{l}\text { Sonderpädagogik und } \\
\text { Rehabilitationswissenschaften }\end{array}$ & $\times$ & - \\
\hline Heidelberg PH & - & BWen & $\times$ & - \\
\hline Heidelberg U & $\begin{array}{l}\text { BW mit dem Schwerpkt. } \\
\text { Organisationsentwicklung }\end{array}$ & $\begin{array}{l}\text { BW mit dem Schwerpkt. } \\
\text { Organisationsentwicklung }\end{array}$ & $x$ & - \\
\hline \multirow[t]{2}{*}{ Hildesheim U } & $\begin{array}{l}\text { EW mit dem Schwerpkt. } \\
\text { Pädagogik der Kindheit/ } \\
\text { Diversity Education }\end{array}$ & $\begin{array}{l}\text { EW mit dem Schwerpkt. } \\
\text { Pädagogik der Kindheit/ } \\
\text { Diversity Education }\end{array}$ & $\times$ & - \\
\hline & $\begin{array}{l}\text { Sozial- und Organisations- } \\
\text { pädagogik }\end{array}$ & $\begin{array}{l}\text { Sozial- und Organisations- } \\
\text { pädagogik }\end{array}$ & $x$ & - \\
\hline \multirow{2}{*}{ Jena U } & $\begin{array}{l}\text { EW - Sozialpädagogik/ } \\
\text { Sozialmanagement }\end{array}$ & $\begin{array}{l}\text { EW - Sozialpädagogik/ } \\
\text { Sozialmanagement }\end{array}$ & $x$ & - \\
\hline & $\begin{array}{l}\text { Bildung - Kultur - Anthro- } \\
\text { pologie }\end{array}$ & $\begin{array}{l}\text { Bildung - Kultur - Anthro- } \\
\text { pologie }\end{array}$ & $x$ & - \\
\hline $\begin{array}{l}\text { Karlsruhe KIT } \\
\text { (TH) }\end{array}$ & Päd & Päd & $x$ & - \\
\hline \multirow[b]{2}{*}{ Karlsruhe PH } & BW & $B W$ & $x$ & - \\
\hline & $\begin{array}{l}\text { Interkulturelle Bildung, Migrati- } \\
\text { on und Mehrsprachigkeit }\end{array}$ & $\begin{array}{l}\text { Interkulturelle Bildung, Migrati- } \\
\text { on und Mehrsprachigkeit }\end{array}$ & $\times$ & - \\
\hline
\end{tabular}




\begin{tabular}{|c|c|c|c|c|}
\hline Standort ${ }^{2}$ & $\begin{array}{l}\text { Studienfachbezeichnung } \\
2014 / 2015\end{array}$ & $\begin{array}{l}\text { Studienfachbezeichnung } \\
2018 / 2019^{3}\end{array}$ & $\begin{array}{l}\text { Ein- } \\
\text { Fach }\end{array}$ & $\begin{array}{l}\text { Zwei- } \\
\text { Fach }^{4}\end{array}$ \\
\hline \multirow{3}{*}{ Kassel U } & $\begin{array}{l}\text { Empirische } \\
\text { Bildungsforschung }\end{array}$ & $\begin{array}{l}\text { Empirische } \\
\text { Bildungsforschung }\end{array}$ & $\times$ & - \\
\hline & $\begin{array}{l}\text { Soziale Arbeit: } \\
\text { Interdisziplinäre Forschung in } \\
\text { Theorie und Praxis }\end{array}$ & $\begin{array}{l}\text { Soziale Arbeit: } \\
\text { Interdisziplinäre Forschung in } \\
\text { Theorie und Praxis }\end{array}$ & $x^{5}$ & - \\
\hline & $\begin{array}{l}\text { Sozialpädagogik in Aus-, Fort- } \\
\text { und Weiterbildung }\end{array}$ & $\begin{array}{l}\text { Sozialpädagogik in Aus-, Fort- } \\
\text { und Weiterbildung }\end{array}$ & $x^{6}$ & - \\
\hline Kiel U & Päd & Päd & $\times$ & $\times$ \\
\hline \multirow[b]{2}{*}{$\begin{array}{l}\text { Koblenz- } \\
\text { Landau U }\end{array}$} & EW & $E W$ & $x$ & - \\
\hline & $\begin{array}{l}\text { EW mit dem Schwerpkt. } \\
\text { Forschung und Entwicklung in } \\
\text { Organisationen }\end{array}$ & $\begin{array}{l}\text { EW mit dem Schwerpkt. } \\
\text { Forschung und Entwicklung in } \\
\text { Organisationen }\end{array}$ & $\times$ & - \\
\hline \multirow{3}{*}{ Köln U } & EW & $E W$ & - & $\times(2)^{\gamma}$ \\
\hline & Rehabilitationswissenschaften & Rehabilitationswissenschaften & $x$ & - \\
\hline & $\begin{array}{l}\text { Interkulturelle Kommunikation } \\
\text { und Bildung }\end{array}$ & $\begin{array}{l}\text { Interkulturelle Kommunikation } \\
\text { und Bildung }\end{array}$ & $\times$ & - \\
\hline \multirow{3}{*}{ Leipzig U } & $\begin{array}{l}\text { Begabungsforschung und } \\
\text { Kompetenzentwicklung }\end{array}$ & $\begin{array}{l}\text { Begabungsforschung und } \\
\text { Kompetenzentwicklung }\end{array}$ & $x$ & - \\
\hline & - & $\begin{array}{l}\text { Professionalisierung früh- } \\
\text { kindlicher Bildung }\end{array}$ & $\times$ & - \\
\hline & - & Early Childhood Research (MSc) & $\times$ & - \\
\hline \multirow{5}{*}{$\begin{array}{l}\text { Ludwigsburg } \\
\mathrm{PH}\end{array}$} & $\begin{array}{l}\text { Frühkindliche Bildung } \\
\text { und Erziehung }\end{array}$ & $\begin{array}{l}\text { Frühkindliche Bildung } \\
\text { und Erziehung }\end{array}$ & $x$ & - \\
\hline & Sonderpädagogik & Sonderpädagogik & $x$ & - \\
\hline & $\begin{array}{l}\text { Bildungsforschung } \\
\text { ( } 3 \text { Semester) }\end{array}$ & $\begin{array}{l}\text { Bildungsforschung } \\
\text { (3 Semester) }\end{array}$ & $x$ & - \\
\hline & $\begin{array}{l}\text { Erwachsenenbildung/ } \\
\text { Weiterbildung }\end{array}$ & $\begin{array}{l}\text { Erwachsenenbildung/ } \\
\text { Weiterbildung }\end{array}$ & $x$ & - \\
\hline & - & Kulturelle Bildung & $x$ & - \\
\hline Lüneburg $U$ & BW - Educational Sciences & BW-Educational Sciences & $\times$ & - \\
\hline \multirow{3}{*}{ Magdeburg U } & BW & $B W$ & $\times$ & - \\
\hline & $\begin{array}{l}\text { Medienbildung: Audiovisuelle } \\
\text { Kultur und Kommunikation }\end{array}$ & $\begin{array}{l}\text { Medienbildung: Audiovisuelle } \\
\text { Kultur und Kommunikation }\end{array}$ & $x$ & - \\
\hline & $\begin{array}{l}\text { International Vocational } \\
\text { Education (MSc) }\end{array}$ & $\begin{array}{l}\text { International Vocational } \\
\text { Education (MSc) }\end{array}$ & $\times$ & - \\
\hline
\end{tabular}

5 Letztmalige Immatrikulation in das 1. Fachsemester

6 Letztmalige Immatrikulation in das 1. Fachsemester

7 Nach Eigendefinition des Standortes ein Studiengang, der in zwei Strukturvarianten angeboten wird: Entweder werden zwei Fächer gleichwertig studiert oder ein Fach als "großes« und das andere als »kleines« Masterfach. 
Tabellenanhang

\begin{tabular}{|c|c|c|c|c|}
\hline Standort ${ }^{2}$ & $\begin{array}{l}\text { Studienfachbezeichnung } \\
2014 / 2015\end{array}$ & $\begin{array}{l}\text { Studienfachbezeichnung } \\
2018 / 2019^{3}\end{array}$ & $\begin{array}{l}\text { Ein- } \\
\text { Fach }\end{array}$ & $\begin{array}{l}\text { Zwei- } \\
\text { Fach }^{4}\end{array}$ \\
\hline Mainz U & $\mathrm{EW}$ & $E W$ & $\times$ & - \\
\hline \multirow[b]{2}{*}{ Marburg U } & E- und BW & $E$ - und $B W$ & $\times$ & - \\
\hline & $\begin{array}{l}\text { Abenteuer- und } \\
\text { Erlebnispädagogik }\end{array}$ & $\begin{array}{l}\text { Abenteuer- und } \\
\text { Erlebnispädagogik }\end{array}$ & $\times$ & - \\
\hline München UBW & $\begin{array}{l}\text { BW, insb. interkulturelle Medien- } \\
\text { und Erwachsenenbildung }\end{array}$ & $\begin{array}{l}\text { BW, insb. interkulturelle Medien- } \\
\text { und Erwachsenenbildung }\end{array}$ & $x$ & - \\
\hline München U & $\begin{array}{l}\text { Päd mit Schwerpkt. } \\
\text { Bildungsforschung und } \\
\text { Bildungsmanagement }\end{array}$ & $\begin{array}{l}\text { Päd mit Schwerpkt. } \\
\text { Bildungsforschung und } \\
\text { Bildungsmanagement }\end{array}$ & $x$ & - \\
\hline Münster U & $\mathrm{EW}$ & $E W$ & $\times$ & - \\
\hline \multirow{2}{*}{ Oldenburg $U$} & E- und BWen & E- und BWen & $x$ & - \\
\hline & - & Rehabilitationspädagogik & $\times$ & - \\
\hline Osnabrück U & $\begin{array}{l}\text { EW: Erziehung und Bildung in } \\
\text { gesellschaftlicher Heterogenität }\end{array}$ & $\begin{array}{l}\text { EW: Erziehung und Bildung in } \\
\text { gesellschaftlicher Heterogenität }\end{array}$ & $x$ & - \\
\hline Paderborn U & EW & $E W$ & - & $x$ \\
\hline Potsdam U & EW & $E W$ & $\times$ & - \\
\hline Regensburg U & $\mathrm{EW}$ & $E W$ & $\times$ & - \\
\hline Rostock U & BW & $B W$ & $\times$ & - \\
\hline \multirow{3}{*}{$\begin{array}{l}\text { Schwäbisch } \\
\text { GmündPH }\end{array}$} & BWen (3 Semester) & BWen (3 Semester) & $\times$ & - \\
\hline & $\begin{array}{l}\text { Interkulturalität und } \\
\text { Integration }\end{array}$ & $\begin{array}{l}\text { Interkulturalität und } \\
\text { Integration }\end{array}$ & $x$ & - \\
\hline & Frühe Bildung & $\begin{array}{l}\text { Kindheits- und Sozial- } \\
\text { pädagogik ( } 3 \text { Semester) }\end{array}$ & $x$ & - \\
\hline Siegen U & Bildung und Soziale Arbeit & Bildung und Soziale Arbeit & $\times$ & - \\
\hline Stuttgart U & - & - & - & - \\
\hline Trier U & EW: Organisation von Wissen & EW: Organisation des Sozialen & $\times$ & - \\
\hline \multirow{5}{*}{ Tübingen U } & $\begin{array}{l}\text { Forschung und Entwicklung } \\
\text { in der Sozialpädagogik/ } \\
\text { Sozialen Arbeit }\end{array}$ & $\begin{array}{l}\text { Forschung und Entwicklung } \\
\text { in der Sozialpädagogik/ } \\
\text { Sozialen Arbeit }\end{array}$ & $\times$ & - \\
\hline & $\begin{array}{l}\text { Erwachsenenbildung/ } \\
\text { Weiterbildung }\end{array}$ & $\begin{array}{l}\text { Erwachsenenbildung/ } \\
\text { Weiterbildung }\end{array}$ & $\times$ & - \\
\hline & $\begin{array}{l}\text { Schulforschung und } \\
\text { Schulentwicklung }\end{array}$ & $\begin{array}{l}\text { Schulforschung und } \\
\text { Schulentwicklung }\end{array}$ & $x$ & - \\
\hline & $\begin{array}{l}\text { Empirische Bildungsforschung } \\
\text { und Pädagogische Psychologie } \\
\text { (MSc) }\end{array}$ & $\begin{array}{l}\text { Empirische Bildungsforschung } \\
\text { und Pädagogische Psychologie } \\
\text { (MSc) }\end{array}$ & $x$ & - \\
\hline & - & $\begin{array}{l}\text { Bildung und Erziehung: } \\
\text { Kultur-Politik - Gesellschaft }\end{array}$ & $x$ & - \\
\hline Vechta U & Soziale Arbeit & Soziale Arbeit & $x$ & - \\
\hline
\end{tabular}




\begin{tabular}{|c|c|c|c|c|}
\hline Standort ${ }^{2}$ & $\begin{array}{l}\text { Studienfachbezeichnung } \\
2014 / 2015\end{array}$ & $\begin{array}{l}\text { Studienfachbezeichnung } \\
2018 / 2019^{3}\end{array}$ & $\begin{array}{l}\text { Ein- } \\
\text { Fach }\end{array}$ & $\begin{array}{l}\text { Zwei- } \\
\text { Fach }^{4}\end{array}$ \\
\hline \multirow{4}{*}{ Weingarten $\mathrm{PH}$} & $\begin{array}{l}\text { Educational Science - BWen } \\
\text { (3 Semester) }\end{array}$ & $\begin{array}{l}\text { Educational Science - BWen } \\
\text { (3 Semester) }\end{array}$ & $\times$ & - \\
\hline & $\begin{array}{l}\text { Medien- und } \\
\text { Bildungsmanagement }\end{array}$ & $\begin{array}{l}\text { Medien- und } \\
\text { Bildungsmanagement }\end{array}$ & $\times$ & - \\
\hline & Early Childhood Studies & Early Childhood Studies & $\times$ & - \\
\hline & - & $\begin{array}{l}\text { Interkulturelle Bildung/ } \\
\text { Kulturvermittlung }\end{array}$ & $\times$ & - \\
\hline \multirow{2}{*}{ Wuppertal U } & $\begin{array}{l}\text { EW: Bildungstheorie und } \\
\text { Gesellschaftsanalyse }\end{array}$ & $\begin{array}{l}\text { EW: Bildungstheorie und } \\
\text { Gesellschaftsanalyse }\end{array}$ & $\times$ & - \\
\hline & $\begin{array}{l}\text { Kindheit, Jugend, } \\
\text { Soziale Dienste }\end{array}$ & $\begin{array}{l}\text { Kindheit, Jugend, } \\
\text { Soziale Dienste }\end{array}$ & $x$ & - \\
\hline \multirow{2}{*}{ Würzburg $U$} & BW & $B W$ & $\times$ & - \\
\hline & Sonderpädagogik & Sonderpädagogik & $\times$ & - \\
\hline
\end{tabular}

** Einfach-Studiengänge mit Nebenfächern $\geq 30$ Leistungspunkten 


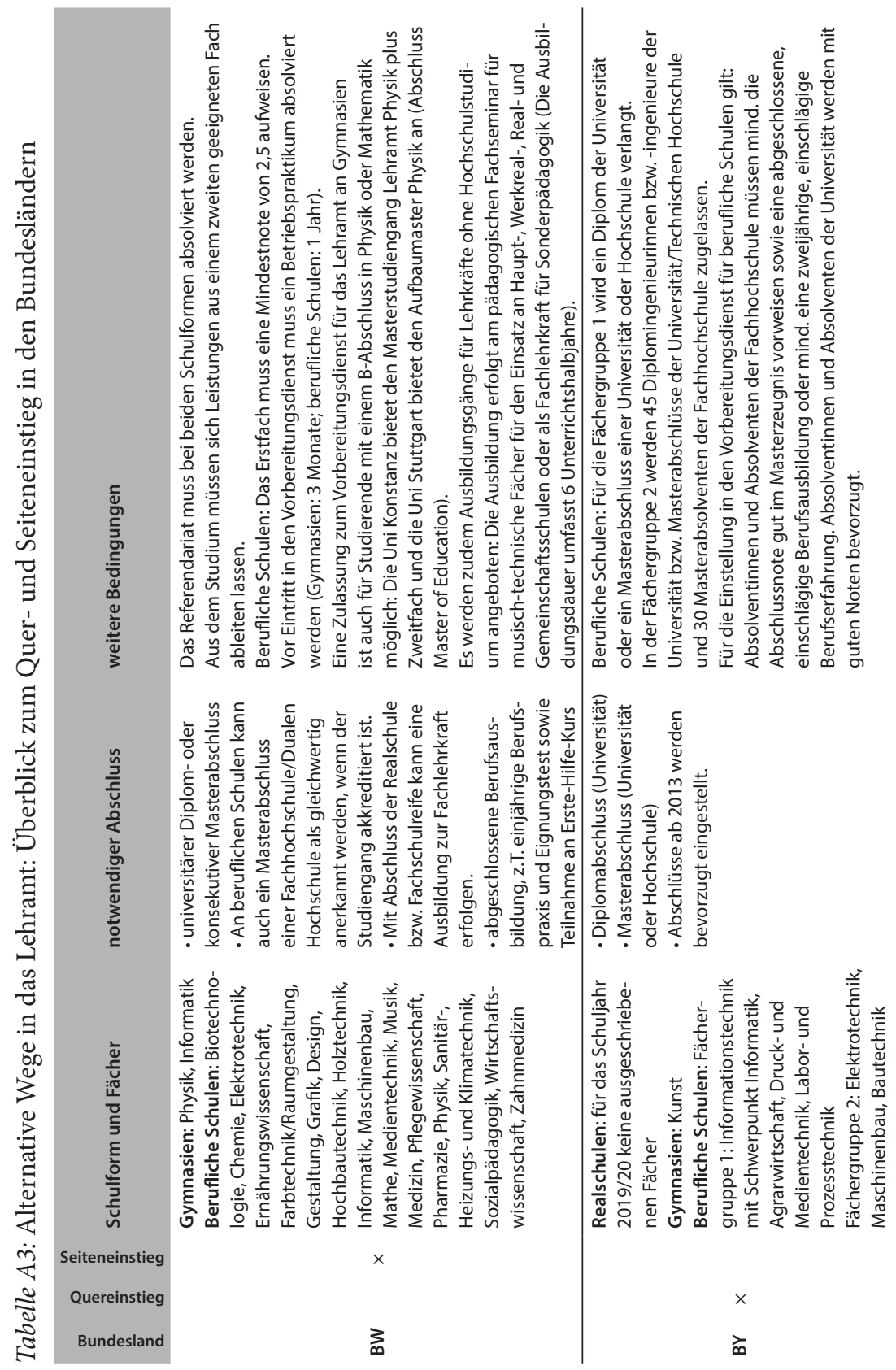




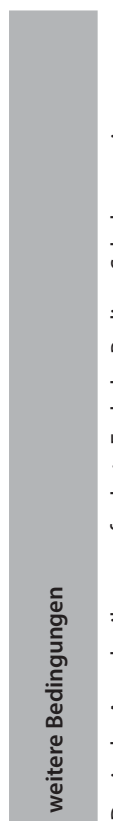

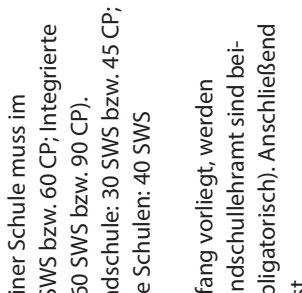

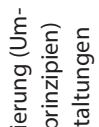

竞高苞苞

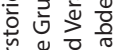

离 造

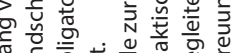

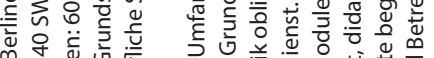

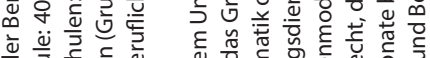

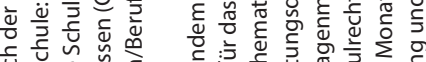

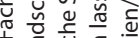

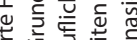

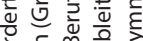

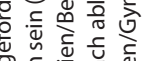

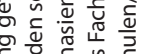

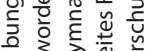

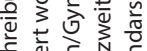

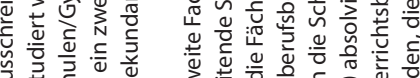

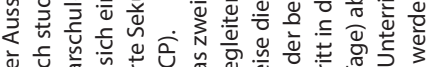

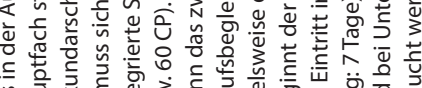

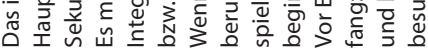

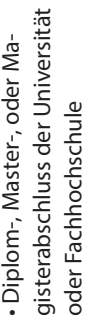

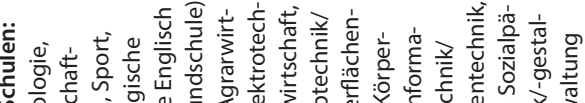

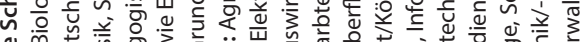

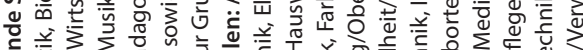

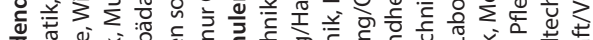

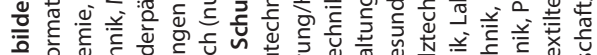

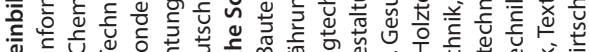

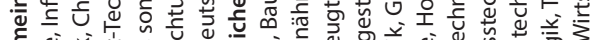

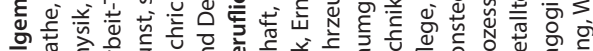

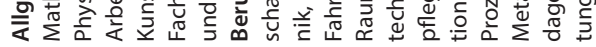

Seiten.

Quer.

Bundes-

land

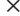

岗
๓

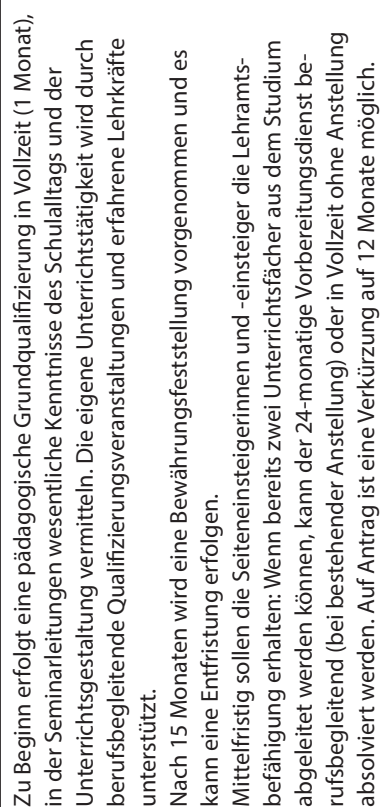

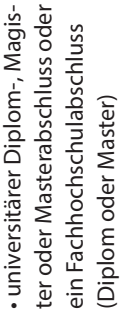
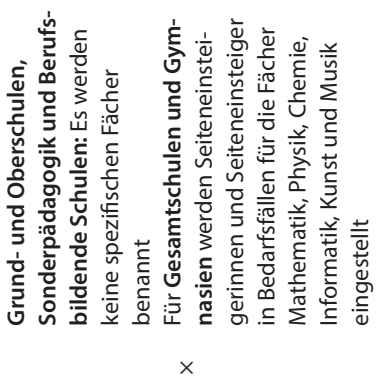

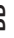




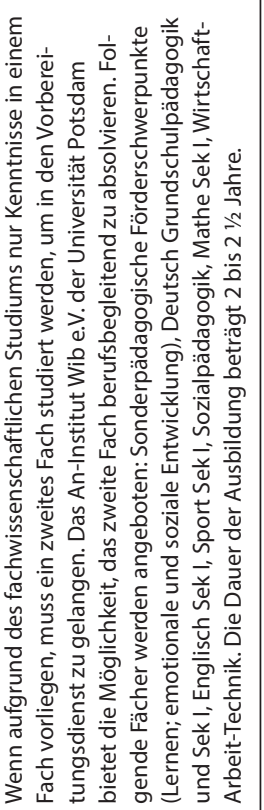

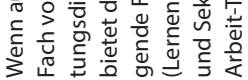

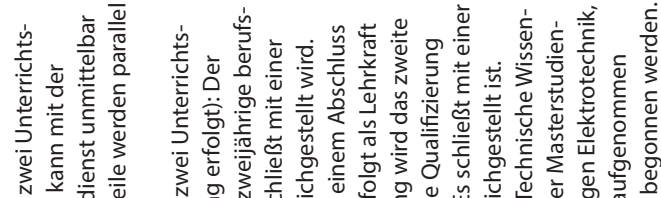

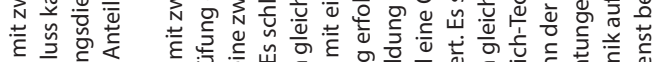

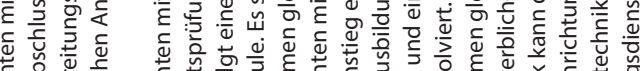

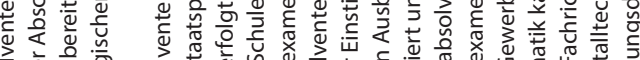

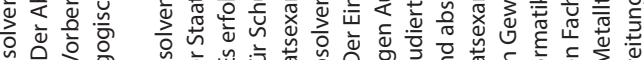

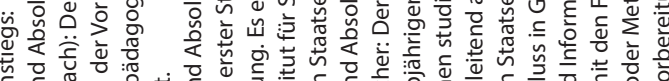

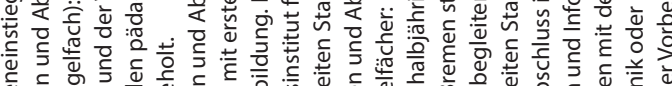

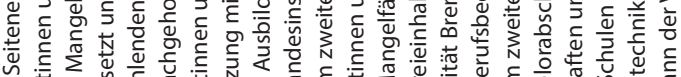

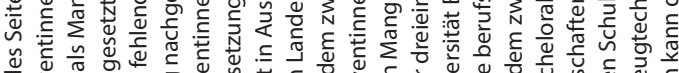

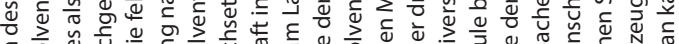

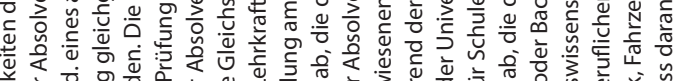

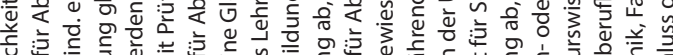

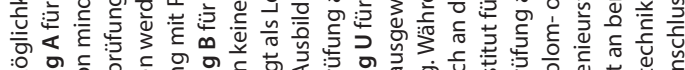
:

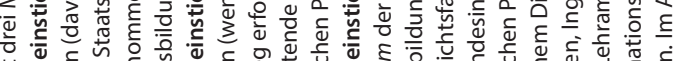

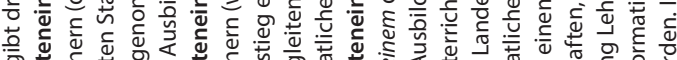

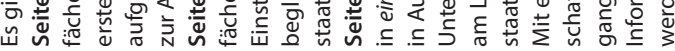

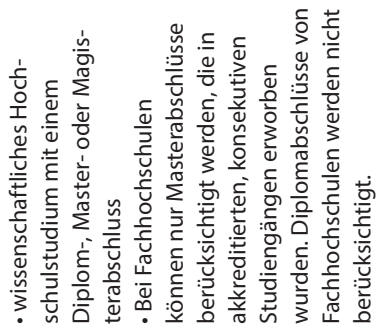

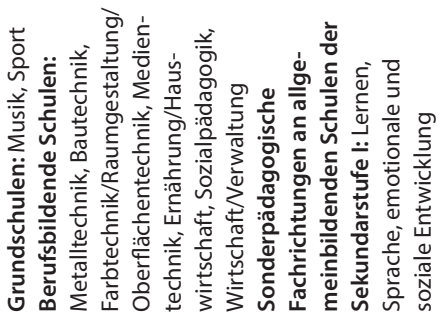

๓ึ

$\dddot{\varphi}$ 


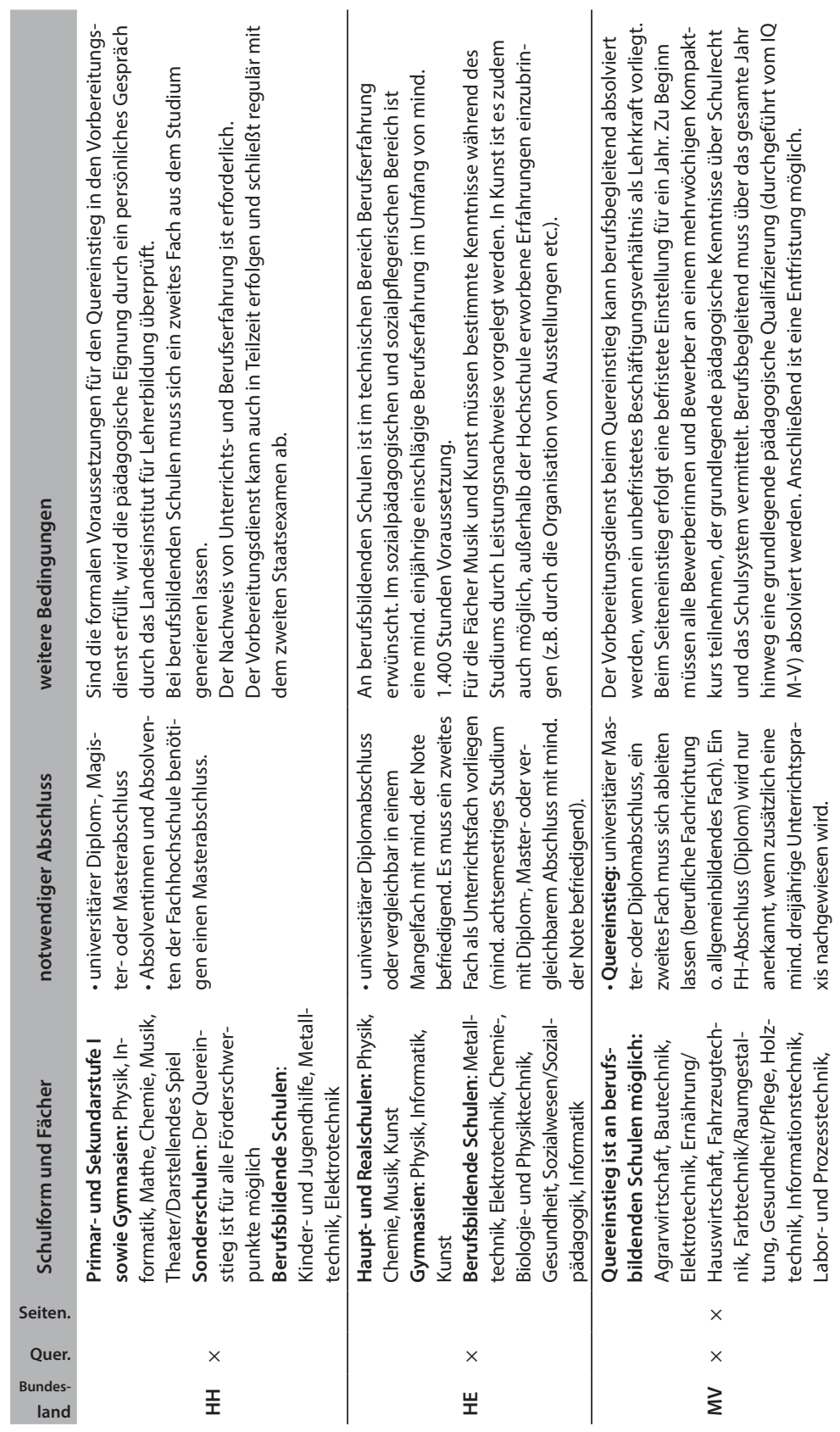




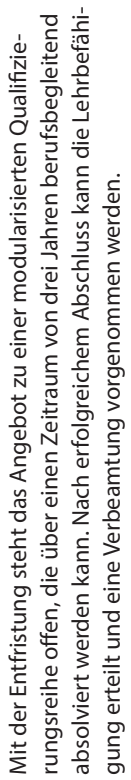

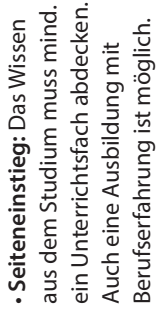
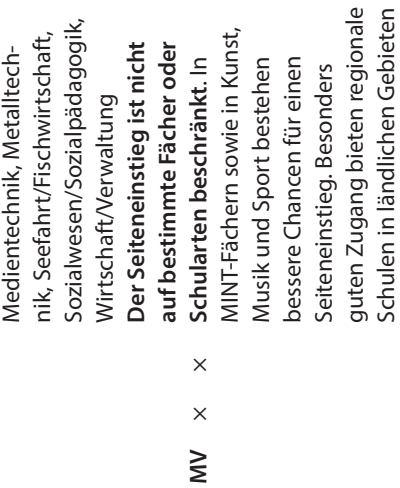

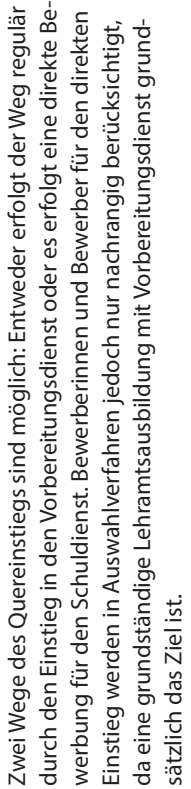

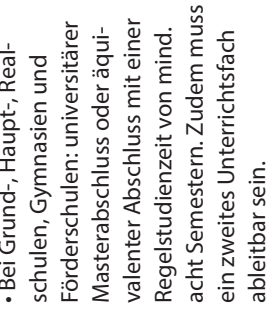

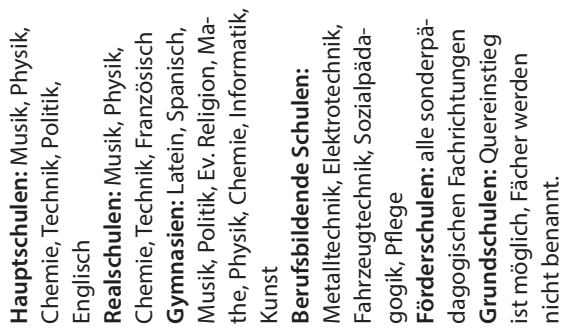

$\bar{z}$ 


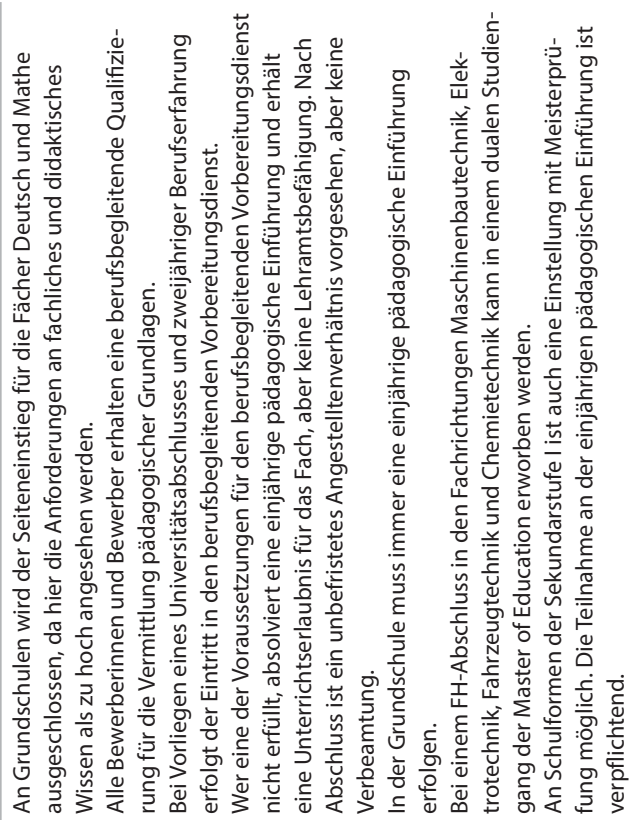

Quer.

Bundes-

land
ह่ं.
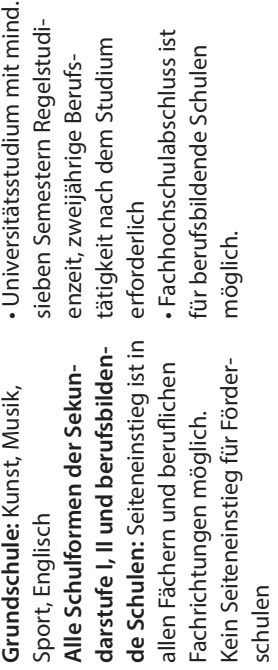

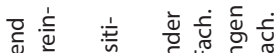

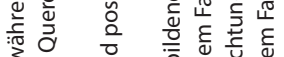
3 ह $\quad$ के

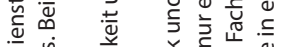

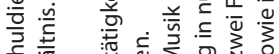

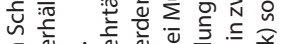
ข .

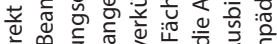
竞

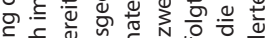

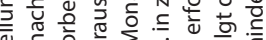

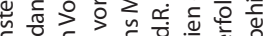
ट⿺辶寸

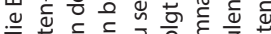

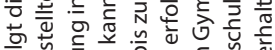

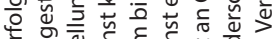
बे

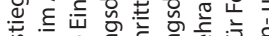

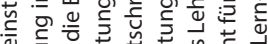
竝

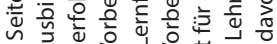
है

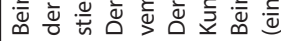

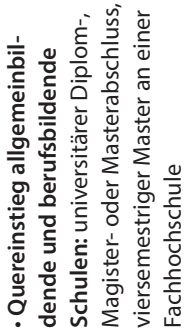

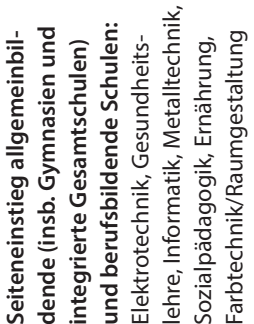

$$
\text { 음 }
$$$$
\times
$$ 


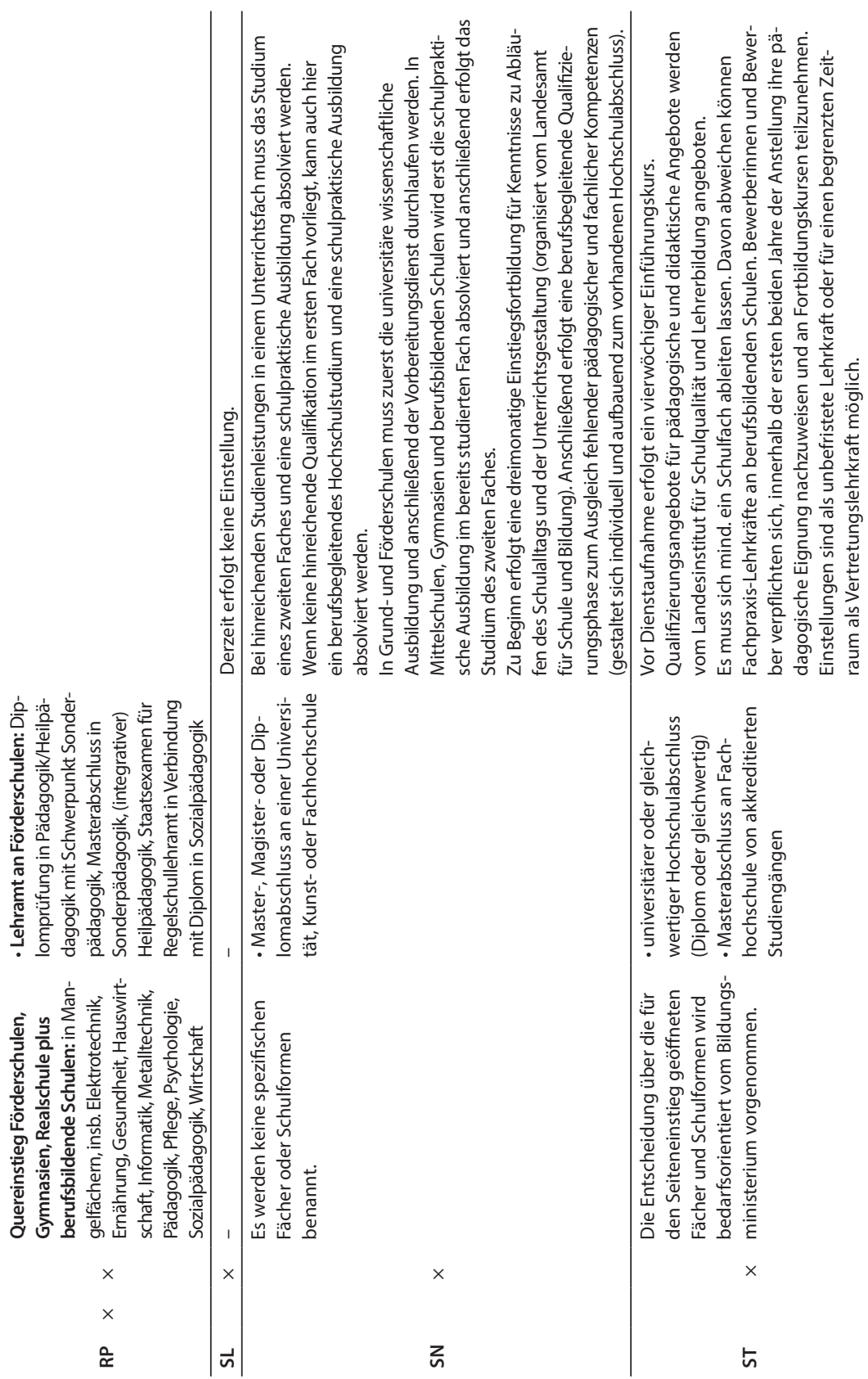



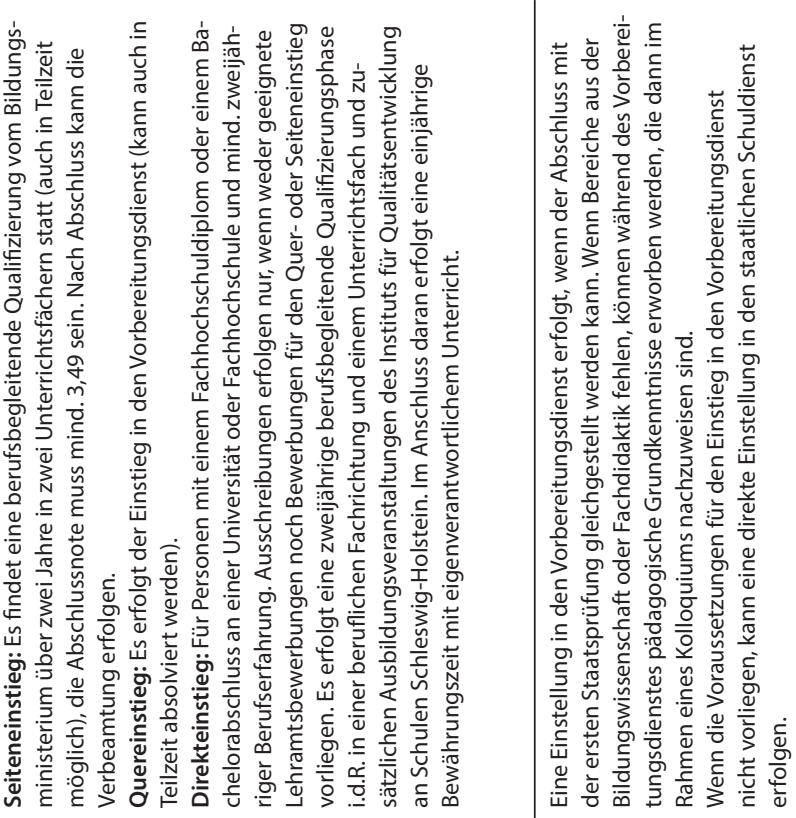

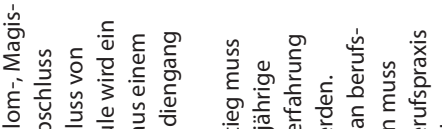
을

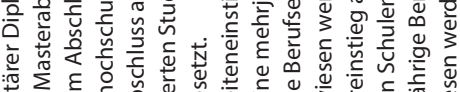

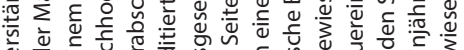

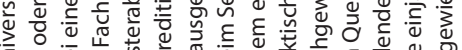

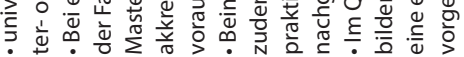

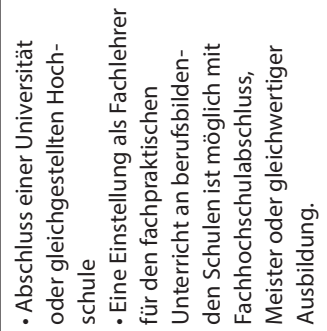

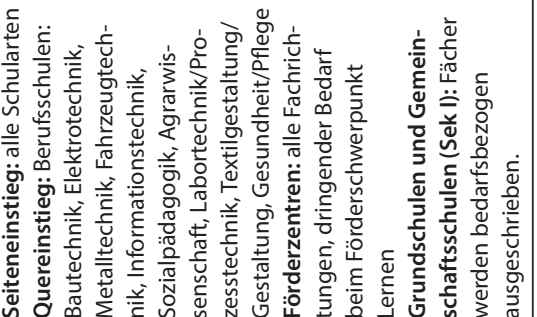
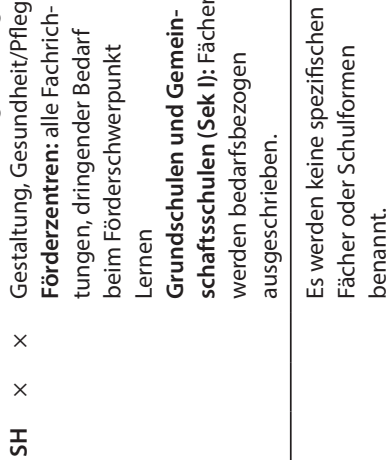


\section{Die Autorinnen und Autoren}

Abs, Hermann Josef, Prof. Dr., Schulpädagoge und Gründungsvorsitzender des Interdisziplinären Zentrums für Integrations- und Migrationsforschung (InZentIM) an der Universität Duisburg-Essen; Arbeitsschwerpunkt: International vergleichende Bildungsforschung

Gerecht, Marius, Dr., wissenschaftlicher Mitarbeiter im Informationszentrum Bildung des DIPF|Leibniz-Instituts für Bildungsforschung und Bildungsinformation in Frankfurt a. M.; Arbeitsschwerpunkte: Forschungsdaten und Forschungsinstrumente der empirischen Bildungsforschung, Bildungsforschung und Bildungsinformation

Grunert, Cathleen, Prof. Dr. phil. habil., Hochschullehrerin für Allgemeine Bildungswissenschaft an der Fernuniversität in Hagen; Arbeitsschwerpunkte: Kindheits- und Jugendforschung, Wissenschaftsforschung, Professionsforschung

Hüfner, Kilian, M. A., Promovend an der Fernuniversität in Hagen und Promotionsstipendiat der Hans-Böckler-Stiftung; Arbeitsschwerpunkte: Kindheitsund Jugendforschung, Kinder- und Jugendkulturen, Migration

Kerst, Christian, Dr., wissenschaftlicher Mitarbeiter am Deutschen Zentrum für Hochschul- und Wissenschaftsforschung (DZHW), Hannover; Arbeitsschwerpunkte: Bildungsberichterstattung, Absolventenstudien, Verhältnis von beruflicher und akademischer Bildung

Krüger, Heinz-Hermann, Prof. Dr. phil. habil., Hochschullehrer für Allgemeine Erziehungswissenschaft an der Martin-Luther-Universität Halle-Wittenberg; Arbeitsschwerpunkte: Bildungs- und Schulforschung, Kindheits- und Jugendforschung, Theorien und Methoden der Erziehungswissenschaft

Kuper, Harm, Univ.-Prof. Dr., Freie Universität Berlin; Arbeitsbereich Weiterbildung und Bildungsmanagement; Vorsitzender der Deutschen Gesellschaft für Erziehungswissenschaft

Ludwig, Katja, Dr. phil., wissenschaftliche Mitarbeiterin am Lehrgebiet Allgemeine Bildungswissenschaft an der Fernuniversität in Hagen; Arbeitsschwerpunkte: Wissenschaftsforschung, Bildungsforschung, Jugendforschung 
Martini, Renate, Dr., wissenschaftliche Mitarbeiterin im Informationszentrum Bildung des DIPF|Leibniz-Institut für Bildungsforschung und Bildungsinformation in Frankfurt a. M.; Arbeitsschwerpunkte: Monitoring der Bildungsforschung, Bildungsinformation, Informationsvermittlung

Müller, Margaretha, M. A.; wissenschaftliche Mitarbeiterin in der Abteilung Erwachsenenbildung/Weiterbildung der Eberhard-Karls-Universität Tübingen; Arbeitsschwerpunkte: Weiterbildungsforschung, Professionalisierung, kulturelle Bildung

Radhoff, Melanie, Dr. phil., Studiengangskoordinatorin der bildungswissenschaftlichen Studiengänge der Fakultät für Kultur- und Sozialwissenschaften an der Fernuniversität in Hagen; Arbeitsschwerpunkte: Selbstwirksamkeit, Professionalisierung von Lehrkräften, Lehrerbildung, Inklusion

Ruberg, Christiane, Dr., Studienrätin im Hochschuldienst am Institut für Allgemeine Didaktik und Schulpädagogik der TU Dortmund; Arbeitsschwerpunkte: Differenz und Schule, Theorie und Geschichte der Schule, Professionalisierung des Lehrerberufs sowie Lehrerbildung und Inklusion

Sauerwein, Markus, Prof. Dr., Fliedner Fachhochschule Düsseldorf; Arbeitsschwerpunkte: Ganztagsschule, Kinder- und Jugendhilfe, Bildungsmonitoring, Internationale Soziale Arbeit

Schmidt-Hertha, Bernhard, Prof. Dr., Professur für Erziehungswissenschaft mit Schwerpunkt berufliche und betriebliche Weiterbildung an der Eberhard-KarlsUniversität Tübingen. Arbeitsschwerpunkte: Bildungsforschung, Lernen und Kompetenzentwicklung im Alter, Informelles Lernen, Hochschulforschung

Schultheiß, Johanna, studentische Hilfskraft am Zentrum für Schul- und Bildungsforschung an der Martin-Luther-Universität Halle-Wittenberg

Stelter, Annette, Dr., wissenschaftliche Mitarbeiterin am Institut für Erziehungswissenschaft an der Justus-Liebig-Universität in Gießen; Arbeitsschwerpunkte: Nachwuchsförderung, Forschungsmethoden in der Aus- und Weiterbildung

Wolter, Andrä, Prof. (i. R.) Dr., Professor für Erziehungswissenschaftliche Forschung zum Tertiären Bildungsbereich an der Humboldt-Universität zu Berlin; Arbeitsschwerpunkte: Hochschulforschung, Lebenslanges Lernen, Bildungsmonitoring 


\section{Hermann Josef Abs, Harm Kuper, Renate Martini (Hrsg.)}

\section{Datenreport Erziehungswissenschaft 2020}

Schriften der Deutschen Gesellschaft für Erziehungswissenschaft (DGfE)

Der Datenreport ist eine regelmäßig alle vier Jahre von der Deutschen Gesellschaft für Erziehungswissenschaft (DGFE) in Auftrag gegebene Darstellung struktureller Entwicklungen der Erziehungswissenschaft. Er informiert die Fachöffentlichkeit, die Bildungs- und Hochschulpolitik sowie die Öffentlichkeit über Trends und den jeweils erreichten Entwicklungsstand der Erziehungswissenschaft als eines der größten Fächer an deutschen Hochschulen. Besonderes Augenmerk liegt auf den Indikatoren zu Studiengängen, Studienabschlüssen, Personal, Forschungsleistungen und Nachwuchsförderung.

Der vorliegende Datenreport bietet Grundinformationen zur Entwicklung der Erziehungswissenschaft über einen Zeitraum von 20 Jahren. Er berücksichtigt Vergleiche zu Nachbardisziplinen und stellt Befunde in den Referenzrahmen allgemeiner Veränderungen im Wissenschaftssystem. Damit dient er der Identifikation fachpolitischer ebenso wie hochschul- und bildungspolitischer Handlungsbedarfe. Relevante Informationen im Datenreport - etwa zu den Studienabschlüssen und den wissenschaftlichen Karrieren - werden nach Geschlechtern differenziert dargestellt. Die DGfE kommt damit ihrem eigenen Anspruch nach, Gendergerechtigkeit zu unterstützen. Der Datenreport ist von herausgehobener Bedeutung für die aktuellen Diskussionen um die Sicherung von Ausbildungskapazitäten für professionelle pädagogische Berufe und um wissenschaftliche Karrieren.

\section{Hrsg.}
Prof. Dr. Hermann Josef Abs
Universität Duisburg-Essen
Prof. Dr. Harm Kuper
Freie Universität Berlin
Dr. Renate Martini
DIPF | Leibniz-Institut für Bildungsforschung und Bildungsinformation, Frankfurt

ISBN 978-3-8474-2419-2

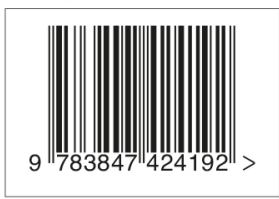

www.budrich-verlag.de 